ALI ALLAHYARZADEH BIDGOLI

Simulation and Optimization of Primary Oil and Gas Processing Plant of FPSO Operating in Pre-Salt Oil Field. 

ALI ALLAHYARZADEH BIDGOLI

Simulation and Optimization of Primary Oil and Gas Processing Plant of FPSO Operating in Pre-Salt Oil Field.

Thesis presented at the Polytechnic School of the University of São Paulo in support of the candidature for the Degree of Doctor in Science of Mechanical Engineering. 
ALI ALLAHYARZADEH BIDGOLI

\section{Simulation and Optimization of Primary Oil and Gas Processing Plant of FPSO Operating in Pre-Salt Oil Field.}

Thesis presented at the Polytechnic School of the University of São Paulo in support of the candidature for the Degree of Doctor in Science of Mechanical Engineering.

Field of Study:

Mechanical Engineering - Energy and Fluids.

Supervised by:

Prof. Dr. Jurandir Itizo Yanagihara 
Este exemplar foi revisado e alterado em relação à versão original, sob responsabilidade única do autor e com a anuência de seu orientador.

São Paulo, ....... de ...................de 2018

Assinatura do autor.....thi alompradeh

Assinatura do orientador

Catalogação-na-publicação

Allahyarzadeh Bidgoli, Ali

Simulation and Optimization of Primary Oil and Gas Processing Plant of FPSO Operating in Pre-Salt Oil Field / A. Allahyarzadeh Bidgoli -- versão corr. -- São Paulo, 2018. $206 \mathrm{p}$.

Tese (Doutrado) - Escola Politécnica da Universidade de São Paulo. Departamento de Engenharia Mecânica.

1.Plataforma offshore de processamento de óleo e gás 2.Análise termodinâmica 3.Análise de sensibilidade 4.Método híbrido 5. Otimização I.Universidade de São Paulo. Escola Politécnica. Departamento de Engenharia Mecânica Il.t. 



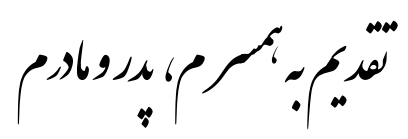

70 my rife and my parents

A minha Familia 



\section{ACKNOWLEDGEMENTS}

I would like to thank my great loving Creator as a First and Foremost Teacher who gave me the thirst for science, the best family, the best teachers and the opportunity to write this thesis. I can do nothing without believing in Him.

I am very much thankful to my wife, for her love, for staying by my side and following my moments of difficulties. I could not complete this research work without your support.

I am extremely appreciative to my parents, for their love, prayers, motivating and sacrifices for educating and preparing me for my future. I would like to extend my thanks to my sisters and also my father and mother-in-law, Mohammad and Maryam.

I would like to express my sincerest thanks and gratitude to my advisor, Prof. Dr. Jurandir Itizo Yanagihara for his advices and guidances. Every time I needed him, he was present and after every discussion, I had a new motivation that helped me to make a significant progress in my work.

I would also like to thank Prof. Silvio de Oliveira Jr., for providing valuable discussions and support during the project.

A special thanks to my friends and colleagues from Universidade de São Paulo: Alencar Migliavacca, Eduardo Suzuki, Felipe Malta, João Gouveia, Prof. Daniel Dezan, Yamid Sanchez, Felipe D’Aloia, Rafael Nakashima, Daniel Flórez-Orrego, Tomas Mora, Milton Gallo, Antonio Fernando Maiorquim, Ehsan Heidaryan, Esther Siroky, Paulo Faggioni Filho e Sidney Carneiro .

I wish to acknowledge the support from PPGEM/POLI/USP, CAPES and BG/Shell Brasil. 

"Buscai o conhecimento, do berço à sepultura!"

Profeta Mohammad (S.A.A.S) 



\section{RESUMO}

As plantas FPSO (Floating, Production, Storage e Offloading), assim como outras plataformas de processamento offshore de petróleo e gás, são conhecidas por terem processos com uso intensivo de energia. Portanto, qualquer aplicação de procedimentos de otimização para consumo de energia e/ou produção pode ser útil para encontrar as melhores condições de operação da unidade, reduzindo custos e emissões de $\mathrm{CO}_{2}$ de empresas que atuam na área de petróleo e gás. Uma planta de processamento primário de uma plataforma FPSO típica, operando em um campo de petróleo em águas profundas brasileiras e em áreas do pré-sal, é modelada e simulada usando seus dados operacionais reais: (i) Teor máximo de óleo / gás (modo 1), (ii) $50 \%$ de teor de BSW no óleo (modo 2) e (iii) teor elevado de água / $\mathrm{CO}_{2}$ no óleo (modo 3). Além disso, uma turbina a gás aeroderivativa (RB211G62 DLE 60Hz) para aplicação offshore é considerada para a unidade de geração da potência eletrica e calor, através dos seus dados reais de desempenho. O impacto de oito parâmetros termodinâmicos de entrada no consumo de combustível e na recuperação de hidrocarbonetos líquidos da unidade FPSO são investigados pelo método SS-ANOVA (Smoothing Spline ANOVA). A partir do SS-ANOVA, os parâmetros de entrada que apresentaram o maior impacto no consumo de combustível e na recuperação de hidrocarbonetos líquidos foram selecionados para aplicação em um procedimento de otimização. Os processos de análise da triagem (usando SS-ANOVA) e de otimização, que consiste em um Algoritmo Híbrido (método NSGA-II + SQP), utilizaram o software Aspen HYSYS como simulador de processo. As funções objetivo utilizadas na otimização foram: minimização do consumo de combustível das plantas de processamento e utilidade e a maximização da recuperação de hidrocarbonetos líquidos. Ainda utilizando SS-ANOVA, a análise estatística realizada revelou que os parâmetros mais importantes que afetam o consumo de combustível da planta são: (1) pressão de saída da primeira válvula de controle (P1); (2) pressão de saída do segundo estágio do trem de separação (e antes da mistura com água de diluição) (P2); (3) pressão de entrada do terceiro estágio do trem de separação (P3); (4) pressão de entrada da água de diluição (P4); (5) pressão de saída do compressor principal de gás $(\mathrm{Pc})$; temperatura de saída de petróleo no primeiro trocador de calor (T1); (7) temperatura de saída de petróleo no segundo trocador de calor (T2); e (8) temperatura da água de diluição. Os parâmetros de entrada de P1, P2, P3 e Pc correspondem a 95\% da contribuição total para a recuperação de hidrocarbonetos líquidos da planta para os modos 1. Analogamente, os três parâmetros de entrada P3, Pc e T2 correspondem a $97 \%$ e $98 \%$ do contribuição total para o consumo de combustível para os modos 2 e 3, respectivamente. Para a recuperação de hidrocarbonetos líquidos da plant, os parâmetros de entrada de P1, P2, P3 e T2 correspondem a 96\% da contribuição total para o consumo de combustível para 
o modo 1. Da mesma forma, os três parâmetros de entrada P3, P2 e T2 correspondem a 97\% e $97 \%$ da contribuição total para a recuperação de hidrocarbonetos líquidos para os modos 2 e 3 , respectivamente. Os resultados do caso otimizado indicaram que a minimização do consumo de combustível é obtida aumentando a pressão de operação no terceiro estágio do trem de separação e diminuindo a temperatura de operação no segundo estágio do trem de separação para todos os modos de operação. Houve uma redução na demanda de potência de 6,4\% para o modo 1,10\% para o modo 2 e 2,9\% para o modo 3 , em comparação com o caso base. Consequentemente, o consumo de combustível da planta foi reduzido em $4,46 \%$ para o modo $1,8,34 \%$ para o modo 2 e $2,43 \%$ para o modo 3 , quando comparado com o caso base. Além disso, o procedimento de otimização identificou uma melhora na recuperação dos componentes voláteis, em comparação com os casos baseline. A condição ótima de operação encontrada pelo procedimento para otimização da recuperação de hidrocarbonetos líquidos apresentou um aumento de 4,36\% para o modo 1 , $3,79 \%$ para o modo 2 e 1,75\% para modo 3, na recuperação líquida de hidrocarbonetos líquidos (e estabilização), quando comparado com as condições operacionais convencionais das suas baseline.

Palavras-chave: Plataforma offshore de processamento de óleo e gás, Análise termodinâmica, Análise de sensibilidade, Método híbrido, Otimização. 


\section{ABSTRACT}

FPSO (Floating, Production, Storage e Offloading) plants, similarly to other oil and gas offshore processing plants, are known to be an energy-intensive process. Thus, any energy consumption and production optimization procedures can be applied to find optimum operating conditions of the unit, saving money and $\mathrm{CO}_{2}$ emissions from oil and gas processing companies. A primary processing plant of a typical FPSO operating in a Brazilian deep-water oil field on pre-salt areas is modeled and simulated using its real operating data. Three operation conditions of the oil field are presented in this research: (i) Maximum oil/gas content (mode 1), (ii) $50 \%$ BSW oil content (mode 2) and (iii) high water/ $\mathrm{CO}_{2}$ in oil content (mode 3). In addition, an aero-derivative gas turbine (RB211G62 DLE 60Hz) with offshore application is considered for the heat and generation unit using the real performance data. The impact of eight thermodynamic input parameters on fuel consumption and hydrocarbon liquids recovery of the FPSO unit are investigated by the Smoothing Spline ANOVA (SS-ANOVA) method. From SS-ANOVA, the input parameters that presented the highest impact on fuel consumption and hydrocarbon liquids recovery were selected for an optimization procedure. The software Aspen HYSYS is used as the process simulator for the screening analysis process and for the optimization procedure, that consisted of a Hybrid Algorithm (NSGA-II +SQP method). The objective functions used in the optimization were the minimization of fuel consumption of the processing and utility plants and the maximization of hydrocarbon liquids recovery. From SS-ANOVA, the statistical analysis revealed that the most important parameters affecting the fuel consumption of the plant are: (1) output pressure of the first control valve (P1); (2) output pressure of the second stage of the separation train before mixing with dilution water (P2); (3) input pressure of the third stage of separation train (P3); (4) input pressure of dilution water (P4); (5) output pressure of the main gas compressor (Pc); (6) output petroleum temperature in the first heat exchanger (T1); (7) output petroleum temperature in the second heat exchanger (T2); (8) and dilution water temperature (T3). Four input parameters (P1, P2, P3 and Pc), three input parameters (P3, Pc and T2) and three input parameters (P3, Pc and T2) correspond to $96 \%, 97 \%$ and $97 \%$ of the total contribution to fuel consumption for modes 1,2 and 3, respectively. For hydrocarbon liquids recovery of the plant: Four input parameters (P1,P2,P3 and T2), three input parameters (P3, P2 and T2) and three input parameters (P3, P2 and T2) correspond to $95 \%, 97 \%$ and $98 \%$ of the total contribution to hydrocarbon liquids recovery for modes 1, 2 and 3, respectively. The results from the optimized case indicated that the minimization of fuel consumption is achieved by increasing the operating pressure in the third stage of the separation train and by decreasing the operating temperature in the second stage of the separation train for all operation modes. There were a reduction in power demand of $6.4 \%$ for mode $1,10 \%$ for mode 2 and $2.9 \%$ 
for mode 3 , in comparison to the baseline case. Consequently, the fuel consumption of the plant was decreased by $4.46 \%$ for mode $1,8.34 \%$ for mode 2 and $2.43 \%$ for mode 3 , when compared to the baseline case. Moreover, the optimization found an improvement in the recovery of the volatile components, in comparison with the baseline cases. Furthermore, the optimum operating condition found by the optimization procedure of hydrocarbon liquids recovery presented an increase of $4.36 \%$ for mode $1,3.79 \%$ for mode 2 and $1.75 \%$ for mode 3 in hydrocarbon liquids recovery (stabilization and saving), when compared to a conventional operating condition of their baseline.

Keywords: Offshore oil and gas processing platform, Thermodynamic analysis, Sensitivity analysis, Hybrid method, Optimization. 


\section{LIST OF FIGURES}

Figure $1.1-$ Photo of the typical FPSO on site $\ldots \ldots \ldots$. . . . . . . . . . 34

Figure 1.2-Gravitational Separator . . . . . . . . . . . . . . . . . . . . . . 35

Figure 1.3-Outline . . . . . . . . . . . . . . . . . . . . . . . . 39

Figure 2.1 - Phase diagram of a typical dry gas reservoir with a line of reduction of reservoir pressure and surface conditions. . . . . . . . . . . . . . .

Figure 2.2 - Phase diagram of a typical wet gas reservoir with a line of reduction of reservoir pressure and surface conditions. . . . . . . . . . . . . . . .

Figure 2.3-Phase diagram of a Condensate gas reservoir with a line of reduction of reservoir pressure and surface separation conditions. . . . . . . . . . .

Figure 2.4 - Phase diagram of a volatile oil reservoir with a line of reduction of reservoir pressure and surface separation conditions. . . . . . . . . . . . . . .

Figure 2.5 - Phase diagram of a black oil reservoir with a line of reduction of reservoir pressure and surface separation conditions. . . . . . . . . . . . . . . 45

Figure 2.6 - Offshore Production Process . . . . . . . . . . . . . . . . . . 47

Figure 2.7 - Global offshore crude oil production, 2005-15 . . . . . . . . . . . . . . 48

Figure 2.8 - Standardized FPSO processing scheme includes basic production separation and treating systems. . . . . . . . . . . . . . . . . 50 50

Figure 2.9 - Overall scheme of a standards FPSOs topside process facilities. . . . . 51

Figure 2.10-Modules of a Replicant FPSO . . . . . . . . . . . . . . . . . . . . 52

Figure 2.11-Simplified overview of an offshore oil and gas platform . . . . . . . . . . 54

Figure 2.12-Oil, gas, $\mathrm{CO}_{2}$ and water production and exports regarding its power demand for the platform during its operation years . . . . . . . . . 56

Figure 2.13-The presented methodology framework in Wang et al. (2012) . . . . . . 62

Figure 2.14-Generic superstructure of an oil and gas processing plant. $\mathrm{S}_{1-2}, \mathrm{~S}_{3-6}$ and $\mathrm{S}_{7-14}$ indicate separation configurations in one, two and three stages, while the numbers indicated for the other steps, e.g. $R_{1}, C_{1}, T_{1}$ and $P_{1}$, are the number of re-compression, compression, treatment and pumping stages, respectively. . . . . . . . . . . . . . . . . . 63

Figure 2.15-The framework of the process optimization with GA . . . . . . . . . . . 66

Figure 3.1 - Relative and global minima. . . . . . . . . . . . . . . . . . . . . . . 81

Figure 3.2-Contours of the objective function in the constraint surfaces in a design space . . . . . . . . . . . . . . . . . . . . . 82

Figure 3.3 - Input variable types in optimization. . . . . . . . . . . . . . . . 83

Figure 3.4-Example of the regular grid (left) and Latin square (right) designs for two-dimensional design with 9 member ensemble. . . . . . . . . . . . 88

Figure 3.5 - LHS designs with significant differences in terms of uniformity. . . . . . . 89 
Figure 3.6 - Incremental Space Filler (ISF). Existing points in the database (previously generated designs) (a), New points are added to fill the space uniformly (b). 90

Figure 3.7 - The Genes, chromosomes and genetic operations. . . . . . . . . . . . . 93

Figure 4.1 - Top view of a typical FPSO unit and all related processes. . . . . . . . . 99

Figure 4.2 -The general scheme of a FPSO . . . . . . . . . . . . . . . 100

Figure 4.3 - Simplified scheme of three-stage separation train. . . . . . . . . . . . 101

Figure 4.4 - Simplified scheme of degasser and electrostatic treatment. . . . . . . . 102

Figure 4.5 -Simplified scheme of Vapor Recovery Unit. . . . . . . . . . . . . . . . 103

Figure 4.6 - Simplified scheme of Main Gas Compression unit. . . . . . . . . . . . . 103

Figure 4.7 - Simplified scheme of Gas Dehydration System. . . . . . . . . . . . . . . 104

Figure 4.8 - Simplified scheme of Hydrocarbons Dew Point Control System. . . . . . 105

Figure 4.9 - Simplified scheme of Exportation Gas Compression unit. . . . . . . . . . 106

Figure 4.10-Simplified scheme of $\mathrm{CO}_{2}$ Removal Unit. . . . . . . . . . . . . . . 107

Figure 4.11-Simplified scheme of $\mathrm{CO}_{2}$ Compression Unit. . . . . . . . . . . . . . . 108

Figure 4.12-Simplified scheme of Injection Gas Compression Unit. . . . . . . . . . . 108

Figure 4.13-Simplified scheme of Gas Turbine and WHRU. . . . . . . . . . . . . . . 109

Figure 4.14-The general scheme of oil and gas processing in FPSO - operational mode 1. . . . . . . . . . . . . . . . . . . . . . . 111

Figure 4.15-The general scheme of oil and gas processing in FPSO - operational

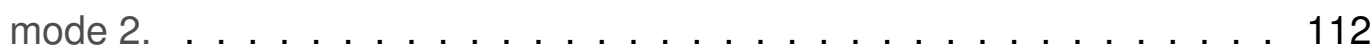

Figure 4.16-The general scheme of oil and gas processing in FPSO - operational mode 3.

Figure 4.17-Conceptual structure of the general methodology and computational steps to perform an optimization procedure for proposed FPSO . . . . . . . . 115

Figure 4.18-How Aspen HYSYS Works . . . . . . . . . . . . . . . . . . . . . 116

Figure 4.19-Sketch of the proposed FPSO with. . . . . . . . . . . . . . . . . . 120

Figure 4.20-Flowchart indicating the processes for screening analyses . . . . . . . . 121

Figure 4.21-Initial population designs checking by statistical tools. . . . . . . . . . . 122

Figure 4.22-General flowchart of the optimization procedure . . . . . . . . . . . . . . 124

Figure 4.23-The procedure of optimization in ModeFRONTIER ${ }^{\text {TM }} \ldots$. . . . . . . . . 125

Figure 5.1 - Power consumption [kW] and percentage for FPSO systems[\%] for operational mode 1. . . . . . . . . . . . . . . . . . . . 130

Figure 5.2 - Power consumption [kW] and percentage for FPSO systems[\%] for operational mode 2. . . . . . . . . . . . . . . . . . . . 130

Figure 5.3 - Power consumption [kW] and percentage for FPSO systems[\%] for operational mode 3.

Figure 5.4-Scatter matrix chart for screening analysis of the input parameters of operation mode $1 \ldots$. . . . . . . . . . . . . . . . . . . 133 
Figure 5.5 - Contribution of the input parameters to hydrocarbon liquids recovery for operation mode 1. . . . . . . . . . . . . . . . . . . . . . . . 134

Figure 5.6 - Total separation efficiency for the conditions shown in Table 5.4 . . . . . 135

Figure 5.7-C7+ molar fraction in exportation oil for the conditions shown in in Table 5.4.136

Figure 5.8-Scatter matrix chart for screening analysis of the input parameters of operation mode 2. . . . . . . . . . . . . . . . . . . . . 137

Figure 5.9-Contribution of the input parameters to hydrocarbon liquids recovery for

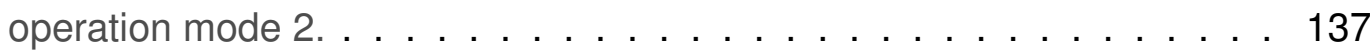

Figure 5.10-Total separation efficiency for the conditions shown in Table 5.5. . . . . . 138

Figure 5.11-Scatter matrix chart for screening analysis of the input parameters of operation mode 2. . . . . . . . . . . . . . . . . . . . . . . . . . . . . . . 139

Figure 5.12-Contribution of the input parameters to hydrocarbon liquids recovery for operation mode 3. . . . . . . . . . . . . . . . . . . 140

Figure 5.13-Contribution of input parameters to fuel consumption in mode 1. . . . . . 142

Figure 5.14-Contribution of input parameters to fuel consumption in mode 2 . . . . . 143

Figure 5.15-Contribution of input parameters to fuel consumption in mode 3. . . . . . 144

Figure 5.16-Contribution of input parameters on FPSO process and utility units. . . . 146

Figure 5.17-SQP convergence curve for the function of hydrocarbon liquids recovery maximization. . . . . . . . . . . . . . . . . . . . . . 147

Figure 5.18-Simplex convergence curve for the objective function of Fuel consumption minimization. . . . . . . . . . . . . . . . . .

Figure 5.19-NSGA-II method convergence curve for the objective function of Fuel consumption minimization.

Figure 5.20-Hybrid method convergence curve for the objective function of Fuel consumption minimization. . . . . . . . . . . . . . . . .

Figure 5.21-The separated gas flow composition in the third stage of separation train for the baseline and optimized cases for operation mode 1.

Figure 5.22-The separated gas flow composition in the third stage of separation train for the baseline and optimized case for operation mode $2 . . . . .$.

Figure 5.23-The separated gas flow composition in the third stage of separation train for the baseline and optimized cases for operation mode 3 . . . . . . . 156

Figure 5.24-Selected point in process steps of separation. . . . . . . . . . . . . . . 158

Figure 5.25-Stabilization of phase during the separation processes for the baseline and optimized cases of operation mode $1 \ldots$. . . . . . . . . . . . . .

Figure 5.26-Stabilization of phase during the separation processes for the baseline and optimized case of operation mode 2.

Figure 5.27-Stabilization of phase during the separation processes for the baseline and optimized case of operation mode $3 . \ldots 165$

Figure A.1 - Efficiency curves by variation of the load. . . . . . . . . . . . . . . . 185 
Figure A.2-Curves of exhausted gas mass flow rate by variation of the load. $\ldots . .186$

Figure A.3 - Curve of the exhausted gas temperature by variation of the load. . . . . 186

Figure A.4-The used worksheet to calculate RB211 off-designs. . . . . . . . . . . . 187

Figure A.5-The performance data of RB211 that regarding the variation of input parameters . . . . . . . . . . . . . . . . 187

Figure A.6 - Applied Gas turbine RB211 performance data in Aspen HYSYS. . . . . 188

Figure B.1-General scheme of proposed FPSO . . . . . . . . . . . . . . . . . . 189

Figure B.2-An overview of simulation of FPSO by Aspen HYSYS . . . . . . . . . . 190

Figure B.3 - Configuration of FPSO plant and utilities plant in this study. . . . . . . . . 191

Figure B.4-Vapor Recovery Unit (VRU) . . . . . . . . . . . . . . . . . . . . . . . . . 191

Figure B.5-Main Gas Compression Unit (MGC) . . . . . . . . . . . . . . . . . . . 192

Figure B.6-Gas Dehydration System and $\mathrm{CO}_{2}$ Removal Unit (GDS\&CO2RU) .. . 192

Figure B.7- $\mathrm{CO}_{2}$ Compression(CO2C) . . . . . . . . . . . . . . . . . . 193

Figure B.8-Exportation Gas Compression(EGC) . . . . . . . . . . . . . . . . . . . . 195

Figure B.9-Injection Gas Compression(IGC) . . . . . . . . . . . . . . . . . . . . 195

Figure B.10-Gas Turbine and Waste Heat Recovery Unit (GT\&WHRU) . . . . . . . . 195

Figure C.1-Couple ModeFrontier with ASPEN HYSYS . . . . . . . . . . . . . . . 197

Figure C.2-Work flow of ModeFrontier for an optimization procedure . . . . . . . . . 198

Figure C.3-Results of SS-ANOVA of hydrocarbon liquids recovery in ModeForntier . 199

Figure D.1 - Hybrid algorithm convergence curve for fuel consumption minimization-

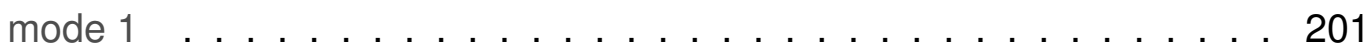

Figure D.2-Hybrid algorithm convergence curve for fuel consumption minimizationmode $2 \ldots \ldots \ldots \ldots$. . . . . . . . . . . . . . . . . . . . . .

Figure D.3-Hybrid algorithm convergence curve for fuel consumption minimizationmode $3 \ldots \ldots . \ldots . \ldots . . \ldots 202$

Figure D.4-Hybrid algorithm convergence curve for hydrocarbon liquids recovery maximization - mode $1 \ldots$. . . . . . . . . . . . . 203

Figure D.5-Hybrid algorithm convergence curve for hydrocarbon liquids recovery maximization - mode $2 \ldots \ldots . \ldots . . \ldots 203$

Figure D.6-Hybrid algorithm convergence curve for hydrocarbon liquids recovery maximization - mode $3 \ldots \ldots$. . . . . . . . . . . . . 204 


\section{LIST OF TABLES}

Table 2.1 - Summary of the main works on the thermodynamics analysis of oil and gas platforms. . . . . . . . . . . . . . . . 57

Table 2.2 - Summary of the main works on the performance of oil and gas platforms. 58

Table 2.3 - Summary of the main works on the performance of oil and gas platforms.

Table 2.4 - Summary of the main works on the process synthesis, sensitivity analysis and optimization of processing plants. . . . . . . . . . . . . . . 74

Table 2.5 - Summary of the main works on the process synthesis, sensitivity analysis and optimization of processing plants. . . . . . . . . . . . . .

Table 2.6 - Summary of the main works on the process synthesis, sensitivity analysis and optimization of processing plants. . . . . . . . . . . . 76

Table 4.1 - Crude oil composition of the three operational modes (molar fraction). . . 113

Table 4.2 - Input parameter ranges and their respective constraints . . . . . . . . . . 119

Table 5.1 - Mass flow rates of the three operational modes. . . . . . . . . . . . . 128

Table 5.2 - Operating pressures and temperatures of streams in the three operational modes. . . . . . . . . . . . . . . . . . . .

Table 5.3 - Operating pressures and temperatures of streams in the three operational modes.

Table 5.4 - Hypothetic conditions to perform separation efficiency . . . . . . . . . . . 135

Table 5.5 - Different pressure operating conditions to perform separation efficiency . 138

Table 5.6 - Input parameters and their respective operating ranges to be used in the maximization of hydrocarbon liquids recovery . . . . . . . . . . . . . . . 141

Table 5.7 - Input parameters and their respective operating ranges to be used in the optimization procedure of fuel consumption minimization . . . . . . . . .

Table 5.8 - Fuel consumption and power demand of the FPSO unit for the baseline and optimized case of operation mode $1 \ldots$. . . . . . . . . . . .

Table 5.9 - Input parameters for the baseline and optimized configurations of operation mode 1. . . . . . . . . . . . . . . . . . . . . . .

Table 5.10-Fuel consumption and power demand of the FPSO unit for the baseline

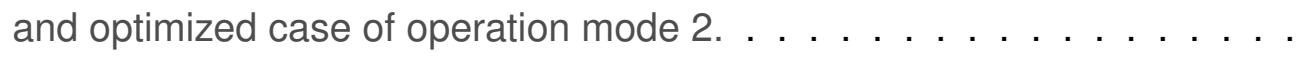

Table 5.11-Input parameters for the baseline and optimized configurations of operation mode $2 \ldots \ldots \ldots \ldots \ldots \ldots$. . . . . . . . . . . . . . . . . .

Table 5.12-Fuel consumption and power demand of the FPSO unit for the baseline

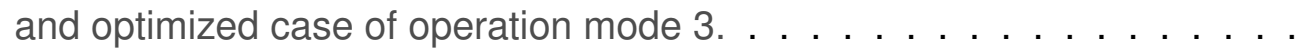

Table 5.13-Input parameters for the baseline and optimized configurations of operation

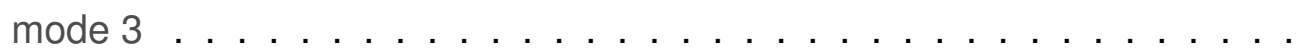


Table 5.14-Input parameters for the baseline and optimized configurations of operation mode 1- Hydrocarbon liquids recovery case. . . . . . . . . . . . . 159

Table 5.15-Exportation oil rate, separation performance of all separators and propane, butane and pentane percentage of exportation oil of the FPSO unit for the baseline and optimized case of operation mode 1 . . . . . . . . . . . . 162

Table 5.16-Input parameters for the baseline and optimized configurations of operation mode 2 - hydrocarbon liquids recovery case. . . . . . . . . . . . . 163

Table 5.17-Exportation oil rate, separation performance of all separators and propane, butane and pentane percentage of exportation oil of the FPSO unit for the baseline and optimized case of operation mode 2. . . . . . . . . . . . 164

Table 5.18-Input parameters for the baseline and optimized configurations of operation mode 3-Hydrocarbon liquids recovery case. . . . . . . . . . . . . 165

Table 5.19-Exportation oil rate, separation performance of all separators and propane, butane and pentane percentage of exportation oil of the FPSO unit for the baseline and optimized case of operation mode 3 . . . . . . . . . . 166

Table B.1 - Required injection pressure as a function of $\mathrm{CO}_{2}$ composition and gas flow. 194 


\section{ACRONYMS}

ABC Air Bottoming Cycle

AfilterSQP Adaptive Filter Sequential Quadratic Programming

ANOVA ANalysis Of VAriance

ANN Anime News Network

API American Petroleum Institute gravity

BSW Basic Sediment and Water

BG British Gas

BORC Basic Organic Rankine Cycle

BP British Petroleum

CAPEX CAPital EXpenditures

CCD Central Composite Design

CCS Carbon Capture and Storage

CMA-ES Covariance Matrix Adaptation Evolution Strategy

CONAMA Conselho Nacional do Meio Ambiente

CO2C $\mathrm{CO}_{2}$ Compression Unit

CO2RU $\mathrm{CO}_{2}$ Removal Unit

C3MR Propane precooled Mixed Refrigerant process

DEA Diethanolamine

DoE Design of Experiment

DOD D-optimal design

DMR Double Mixed Refrigerant

EA Evolutionary Algorithms

EGC Exportation Gas Compression unit 
EIA U.S. Energy Information Administration

EOR Enhance Oil Recovery

EOS Equation of State

FLNG Floating Liquefied Natural Gas

FPSO Floating, Production, Storage and Offloading

FWKO Free Water Knockout

GA Genetic Algorithm

GAMS The General Algebraic Modeling System

GDP Gross Domestic Product

GDS Gas Dehydration System

GHG GreenHouse Gas

GOR Gas-to-Oil Ratio

GT Gas Turbine

HE Heat Exchanger

HX Heat Exchanger

HDP Hyrocarbon Dew Point control system

HRVG Heat Recovery Vapor Generator

HVAC Heating, Ventilation and Air Conditioning

IGC Injection Gas Compression unit

IOGP The International Association of Oil \& Gas Producers

ISF Incremental Space Filler

KanGAL Kanpur Genetic Algorithms Laboratory

KBO Knowledge-Based Optimization

KSMR Korea Single Mixed Refrigerant

LEC Levelized Energy Cost

LH Latin Hypercube 
LHS Latin Hypercube Sampling

LHV Lower Heating Value

LNG Liquefied Natural Gas

MCD Coordinate Descent Methodology

MDEA Methyldiethanolamine

MF ModeFRONTIER ${ }^{\text {TM }}$

MGC Main Gas Compression unit

MILP Mixed-Integer Linear Programming

MINLP Mixed-Integer Non-Linear Programming

MIPSQP Mixed Integer Programming Sequential Quadratic Programming

MOPSO Multi Objective Particle Swarm Optimizer

MRC Mixed Refrigerant Cycle

NCS Norwegian Continental Shelf

NG Natural Gas

NGL Natural Gas Liquid

NLP NonLinear Programming

NNA Nearest Neighbor Algorithm

NMPC Non Linear Model Predictive Control

NSGA Non-dominated Sorting Genetic Algorithm

OAD Orthogonal Array Design

OPEX OPerating EXpenditure

ORC Organic Rankine Cycle

PSO Particle Swarm Optimization

PDF Probability Density Function

RORC Regenerative Organic Rankine cycle

RSM Response Surface Methodology 
PR Peng-Robinson

SG Specific Gravity

SQP Sequential Quadratic Programming

SS-ANOVA Smoothing Spline-ANalysis Of VAriance

ST Separation Train

St.Dev. Standard Deviation

TGC Technology Global Center

UD Uniform Design

ULH Uniform Latin Hypercube

VBA Visual Basic for Applications

VBScript Microsoft Visual Basic Scripting Edition

VRU Vapor Recovery Unit

WAG Water Alternating Gas

WHEN Work-Heat Exchanger Networks

WHRU Waste Heat Recovery Unit

WOR Water to Oil Ratio 
NOTATIONS

\begin{tabular}{|c|c|}
\hline \multicolumn{2}{|l|}{ Symbols } \\
\hline$C 1$ & Methane \\
\hline$C 2$ & Ethan \\
\hline$C 3$ & Propane \\
\hline$C 4$ & Butane \\
\hline$C 5$ & Pentane \\
\hline$C 6$ & Hexane \\
\hline$C 7$ & Heptane \\
\hline$C 8$ & Octane \\
\hline$h$ & Specific enthalpy \\
\hline$L H V$ & Lower heating value \\
\hline$\dot{m}$ & Mass flow rate \\
\hline$\dot{n}$ & Molar flow rate \\
\hline$P$ & Pressure \\
\hline$\dot{Q}$ & Heat \\
\hline$S G$ & Specific gravity \\
\hline$T$ & Temperature \\
\hline$\dot{W}$ & Power \\
\hline \multicolumn{2}{|c|}{ Greek symbols } \\
\hline$\kappa_{k}$ & Collinearity index \\
\hline$\eta$ & Efficiency \\
\hline$\nu$ & Volume \\
\hline \multicolumn{2}{|c|}{ Subscripts } \\
\hline air & Air \\
\hline$c c$ & Combustion chamber \\
\hline Feed & Feed crude oil \\
\hline$G T$ & Gas Turbine \\
\hline Heavy & Heavy Hydrocarbons \\
\hline in & Input \\
\hline$i$ & i-th component \\
\hline Light & Light Hydrocarbons \\
\hline Medium & Medium Hydrocarbons \\
\hline net & Net \\
\hline out & Output \\
\hline $\operatorname{sep}$ & Separation \\
\hline
\end{tabular}





\section{CONTENTS}

INTRODUCTION ..................... 31

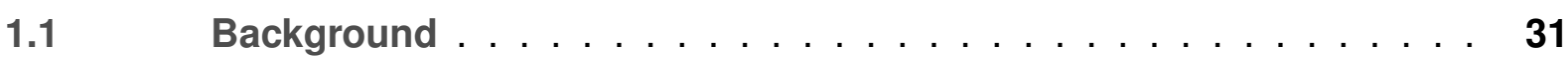

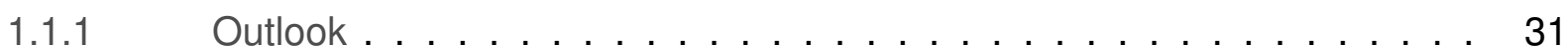

$1.2 \quad$ Primary (Petroleum) Processing Plant of FPSO . . . . . . . . . . 33

1.2.1 Primary Separation Train of Petroleum . . . . . . . . . . . . . . . . . . 34

1.2.2 Gas compression treatment, re-injection and exportation system . . . . . 35

1.2.3 $\quad \mathrm{CO}_{2}$ Removal, Compression and Injection System . . . . . . . . . . 36

$1.3 \quad$ FPSO operational modes . . . . . . . . . . . . . . 36

1.3.1 Operational mode $1 \ldots \ldots \ldots \ldots$

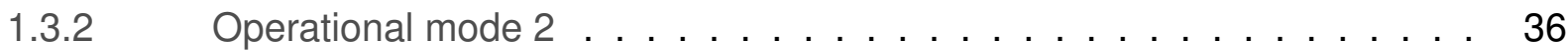

1.3.3 Operational mode $3 \ldots \ldots \ldots \ldots$

$1.4 \quad$ Motivation . . . . . . . . . . . . . . . 37

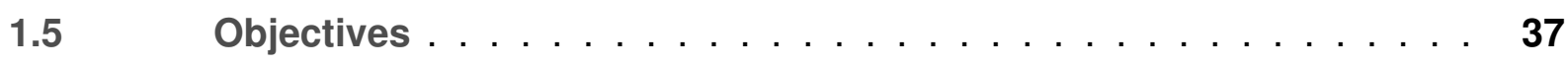

$1.6 \quad$ Outline of the Thesis . . . . . . . . . . . . . . 38

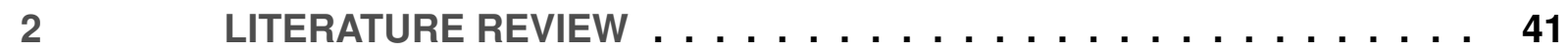

$2.1 \quad$ Primary Petroleum and Gas Processing Offshore Platform ～. . . . 41

2.1.1 Composition, Crude Oil, Gas and Reservoir Fluid . . . . . . . . . . . . 41

2.1.2 Offshore Platforms: Processes and Configurations . . . . . . . . . . . 45

2.1.3 Brazilian Reservoir, Offshore industry and FPSO Configurations . . . . . 48

2.1.4 Thermodynamics analysis of oil and gas processing plant . . . . . . . 52

$2.2 \quad$ Process Optimization in Processing Plants $\ldots \ldots$. . . . . . . 60

2.2.1 Process Synthesis and Modeling . . . . . . . . . . . . . . . . 60

2.2.2 Sensitivity analysis and Optimization of Industrial Processing Plant . . . 64

2.2.3 Sensitivity analysis and Optimization of Oil and Gas Processing Plants . 69

$2.3 \quad$ Overview . . . . . . . . . . . . . . . . . . . 77

3 THEORETICAL FOUNDATION . . . . . . . . . . . . . . . 79

$3.1 \quad$ Thermodynamic Analysis . . . . . . . . . . . . . . . 79

3.1.1 Separation Performance . . . . . . . . . . . . . . . . 80

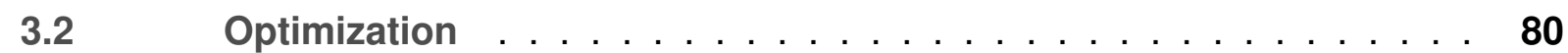

3.2.1 Algorithmic methods . . . . . . . . . . . . . . . . 83

3.2.2 Classification of algorithmic optimization methods . . . . . . . . . . . . 84

3.2.3 Screening Analysis . . . . . . . . . . . . . . . . 86

3.2.4 Design of Experiments $(\mathrm{DoE}) \ldots \ldots \ldots$. . . . . . . . . . . 86 
3.2.4.1 Latin hypercube sampling (LHS), Uniform Latin Hypercube (ULH) and Incremental Space Filler (ISF) . . . . . . . . . . . . . . . . . . . . . . 88

3.2.4.2 Smoothing spline ANOVA method (SS-ANOVA) . . . . . . . . . . . . . . . . . 90

3.2.5 Genetic Algorithms - Fundamentals . . . . . . . . . . . . . . . . . . 93

3.2.5.1 Non-dominated sorting genetic algorithm II (NSGA-II) . . . . . . . . . . . . . . . . 94

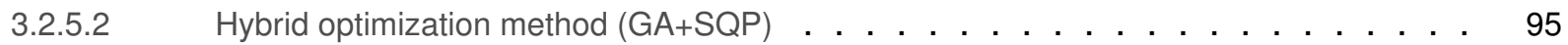

3.2.6 Sensitivity of the optimum . . . . . . . . . . . . . . . . . 96

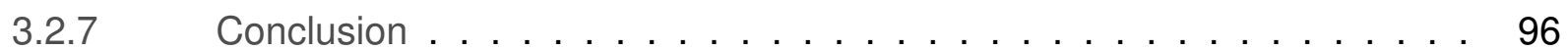

4 METHODOLOGY IMPLEMENTATION . . . . . . . . . . . . . . . . 99

$4.1 \quad$ System description and simulation $\ldots \ldots \ldots$

4.1.1 Separation Train . . . . . . . . . . . . . . . . . . . 100

4.1 .2 Gas Treatment Units . . . . . . . . . . . . . . . . . . . . . 102

4.1.2.1 Vapor Recovery Unit (VRU) . . . . . . . . . . . . . . . . . . . . . . . . . . 102

4.1.2.2 Main Gas Compression (MGC) . . . . . . . . . . . . . . . . . . . . . . . . . 103

4.1.2.3 Gas dehydration System (GDS) . . . . . . . . . . . . . . . . . . . . . 104

4.1.2.4 Hydrocarbons Dew Point Control System (HDP) . . . . . . . . . . . . . . . . . . 104

4.1.2.5 Exportation Gas Compression System (EGC) ． . . . . . . . . . . . . . . . . . . 105

$4.1 .3 \quad \mathrm{CO}_{2}$ Treatment Units . . . . . . . . . . . . . . . . . . . . . . 106

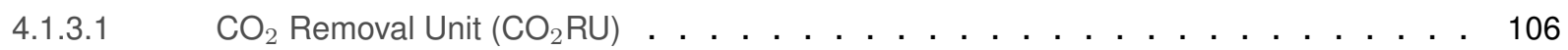

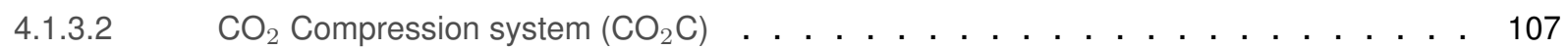

4.1.3.3 Injection Gas Compression unit(IGC) . . . . . . . . . . . . . . . . . . . . . . . 108

$4.1 .4 \quad$ Utility plants . . . . . . . . . . . . . . . . . . . 109

4.1.4.1 Power and heat generation unit . . . . . . . . . . . . . . . . . . . . . . 109

4.1.4.2 Hot Water system . . . . . . . . . . . . . . . . . . . . . . . . . 110

4.1.4.3 Cooling Water system . . . . . . . . . . . . . . . . . . . . . . . . 110

4.1.4.4 Water Injection System . . . . . . . . . . . . . . . . . . . . . . . . 110

4.1 .5 Operation Scenarios . . . . . . . . . . . . . . . . . . . . . . . . 111

4.2 Assumptions for modeling and simulation . . . . . . . . . . . . 113

$4.3 \quad$ Modeling, Simulation and optimization description . . . . . . . . . 114

$4.3 .1 \quad$ Strategy . . . . . . . . . . . . . . . . . . . . . 114

4.3.2 Simulation of FPSO in HYSYS . . . . . . . . . . . . . . . . . . . . . . . . . 115

4.3.3 Sensitivity analysis description . . . . . . . . . . . . . . . . . . . . 118

4.3.4 Optimization description . . . . . . . . . . . . . . . . . . . 123

4.3.4.1 Optimization in ModeFRONTIER ${ }^{\mathrm{TM}}$. . . . . . . . . . . . . . . . . . . . . . . . . . . . . . . . . 124

$5 \quad$ RESULTS AND DISCUSSIONS $\ldots \ldots \ldots \ldots \ldots \ldots \ldots$

5.1 Thermodynamic analysis results . . . . . . . . . . . 127

$5.2 \quad$ Sensitivity analysis results $\ldots \ldots \ldots \ldots \ldots \ldots \ldots$

5.2.1 Contribution of Input Parameters on Hydrocarbon Liquids Recovery . . . 131 


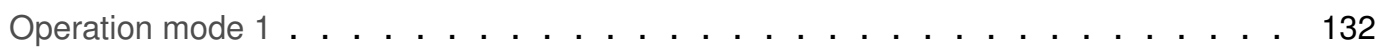

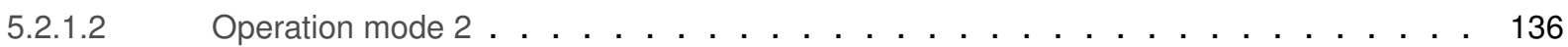

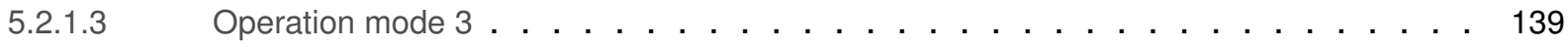

5.2.2 The contribution of Input Parameters To Fuel Consumption . . . . . . . . 141

5.2.2.1 Operation mode 1. . . . . . . . . . . . . . . . . . . . . . . . . 141

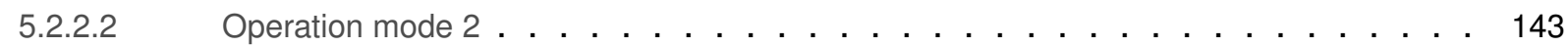

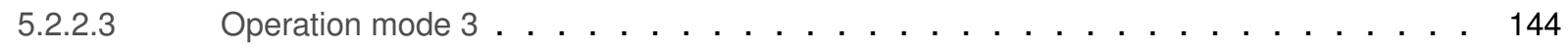

$5.3 \quad$ Optimization Results . . . . . . . . . . . . . . . . . . 146

5.3.1 Assessment of applied optimization methods . . . . . . . . . . . 146

$5.3 .2 \quad$ Fuel consumption . . . . . . . . . . . . . . . . . . 150

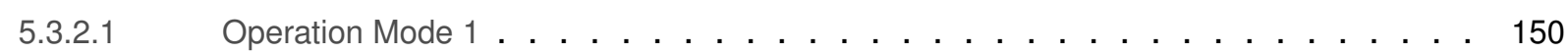

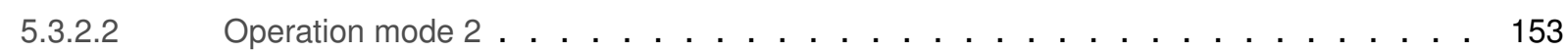

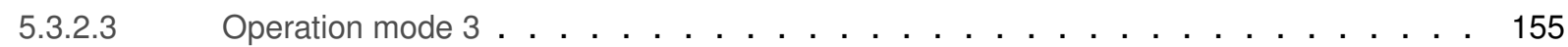

5.3.3 Hydrocarbon liquids recovery . . . . . . . . . . . . . . . . 157

5.3.3.1 Operation mode 1. . . . . . . . . . . . . . . . . . . . . . . . . . . 158

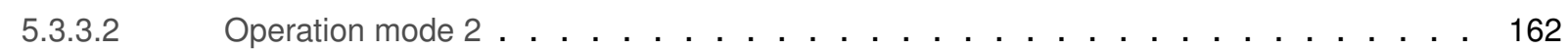

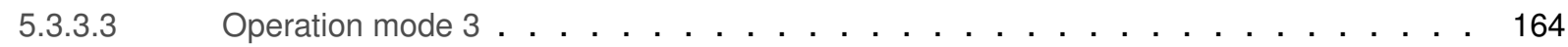

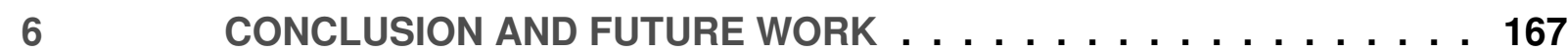

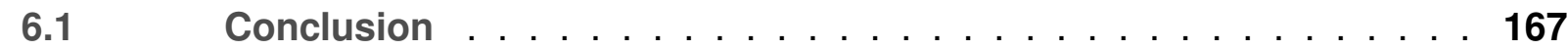

$6.2 \quad$ Future work . . . . . . . . . . . . . . . . . . 169

REFERENCES $\ldots \ldots \ldots \ldots \ldots \ldots \ldots \ldots \ldots \ldots$

APPENDIX A - MODELING AND SIMULATION OF RB211G62 DLE 6OHZ TURBINE IN GATECYCLE AND USING THIS DATA IN ASPEN HYSYS . . . . . . . . . . . . 185

APPENDIX B - MODELING AND SIMULATION SIMULATOR ILLUSTRATION OF FPSO BY ASPEN HYSYS $\ldots \ldots$. . . . . . . 189

APPENDIX C - COUPLING MODEFRONTIER WITH ASPEN HYSYS . 197 APPENDIX D - CONVERGED OBJECTIVE FUNCTIONS . . . . 201 



\section{INTRODUCTION}

\subsection{Background}

\subsubsection{Outlook}

Petroleum has been used since ancient times. About 4000 years ago, it was utilized in Babylon as a material for building walls and towers. Ancient Persian tablets also indicate medicinal and lighting applications of petroleum at the higher levels of society (Chisholm, Hugh, 1911). However, oil is important in the Energy Matrix, but it currently has an inevitable role across society, concerning environmental pollution, economy, geopolitics, and technology. After many decades, petroleum is still one of the most important fossil fuels. New resources, such as shale gas besides shale oil, tar sand, pre-salt oil and condensate and heavy oil are also of interest for exploitation. The increase in the world energy use is planned to reach $56 \%$ in the next three decades, which is considered mainly a result of population growth and rising prosperity in developing countries (U.S. ENERGY INFORMATION ADMINISTRATION, 2013). In the last annual report of EIA in 2018 (U.S. Energy Information Administration (EIA), 2018), the projected gross domestic product (GDP) of the world from 2017 is dependent on hydrocarbons fuel and natural gas accounts for the largest share of the total energy production.

According to the statistical report published in 2015 by British Petroleum, Brazil is the eighth largest energy consumer in the world and, behind the United States and Canada, it is the third largest in the Americas. Most of this energy consumption involves oil and other liquid fuels, followed by hydropower and natural gas. Due to the discovery of new Brazilian pre-Salt fields, the reservoirs have expanded from 15 billion barrels of oil in 2004 to more than 30 billion in 2009, making Brazil a top 10 liquid fuel producer in the world. In 2014, Brazil produced a large amount of oil, about 2.95 million barrels per day (b/d), representing a $9.5 \%$ increase as compared to 2013. Fossil fuels such as oil, natural gas and condensate production represent about $60 \%$ of the Brazilian energy matrix and increasing domestic oil and gas production has been a long-term objective of the Brazilian government. In turn, Brazil is identified as the world's 7th-largest emitter of greenhouse gases and as the third largest emitter after China and India among the developing countries. The oil and gas exploration and production industries emit a considerable percentage of greenhouse gases and are energy intensive. Some countries were therefore compelled to promote the mitigation of contamination and the common proposal is to lower the $\mathrm{CO}_{2}$ rate. The reduction in $\mathrm{CO}_{2}$ emissions is hence an important factor in industrial development (LOUREIRO et al., 2013)(PB, 2015)(Ministério de Minas e Energia, 2015). 
Hence, there are the following important challenges that need addressing for the energy strategy of any oil and gas industrial:

- Efficiency challenge (developing and improving the applied thermal systems in the oil and gas industry regarding crude oil compositions and operating conditions).

- Environmental impact and sustainability challenges (reduction in energy consumption and/or reduction in the environmental effect of oil and processing plants).

The first issue may be addressed by carrying out a precise system analysis to improve and to optimize the thermal efficiency and performance of diverse energy- consuming processes (power and heating).

The second one can be solved by first, mitigating $\mathrm{CO}_{2}$ emission in oil and gas processing, including $\mathrm{CO}_{2}$ content of oil and gas compositions. Second, reducing the required power demand leads to less total fuel consumption of an oil and gas processing plant.

This environmental purpose is a sustainability requirement of technological planning comprising both processing and utility plants. Note that sustainable proposals should also be developed for offshore processes, including security demands, reliability, besides the demands of size and weight increment, especially comparing offshore-type processes to onshore processes (REAY et al., 2013). Moreover, along with the two challenges considered, the profitability of the system, including increasing oil and gas production can play an effective role to encourage companies to mitigate the environmental impacts.

Oil and gas production and processing in offshore platforms are an important sector of the global oil industry. These platforms have been configured in two plants, which comprise processing plants and utility units. The main plant is responsible for separating oil from associated gas, water, salt, and for processing the desired production. The utility plants are where air, fuel gas, cooling and heating water are used.

A typical oil and gas offshore installation, may contain the following systems (NGUYEN et al., 2013):

- Production manifolds;

- Oil separation;

- Oil pumping and exportation;

- Re-compression and gas purification;

- Gas compression and exportation;

- Wastewater treatment;

- Sea water injection; 
- Power and heat generation unit;

- HVAC and other utilities.

The power generation unit is responsible for the consumption of the plant itself and a number of important considerations could involve diagrams and a power generation planning scheme in conjunction with the process scheme as follows: conditions and standards of the production process, available technologies, energy analysis methods, dynamic manufacturing process. Furthermore, utilities must seek the best options in terms of the arrangement, capacity, type and number of machines, to ensure an adequate economic/financial return and reasonable operation to meet efficient operation and the project requirements (BALESTIERI, 2002).

In addition to the indicated technological options available, many studies can be implemented in the production process of offshore platforms for sustainability. In some oil offshore platform processes, water is required and this process permits, for example, capturing the water contained in the gas combustion of a gas turbine. This is a potential water source for this type of applications (NGUYEN et al., 2013). Furthermore, in crude oil with considerable $\mathrm{CO}_{2}$ content, the separated $\mathrm{CO}_{2}$ should be stored (because of environmental issues) or injected into the well as EOR (enhance oil recovery) and for an offshore plant, the separated gas cannot be sent to the flare (ARAÚJO et al., 2017).

\subsection{Primary (Petroleum) Processing Plant of FPSO}

A wide variety of offshore installations have been used throughout the world, and the most suitable offshore plants for deep-water are floating platforms. The FPSO (Floating Production, Storage and Offloading) units have a technical advantage for the short-lived well exploration and the remote marginal field, whereby fixed offshore installations are impractical and whereby building a pipeline is cost-prohibitive (GEHLING et al., 1994) (KINNEY P.E., 2012). FPSOs are useful in oil regions, which do not have a pipeline infrastructure in that place and a storage tank does not need to be idle while a processing facility produces enough oil to fill it. In addition, the advantage of those FPSOs over the pipelines is that once an oil field has been exhausted, the vessel can be moved to another location. There are currently about 200 of such vessels operating worldwide. Figure 1.1 shows a typical FPSO on site.

In Brazil, petroleum is one of the main industries. The offshore exploration in Brazil is located in the Santos Basin in the south and the Espirito Santo Basin in the north, where the salt layer has a thickness ranging from 200 to $2000 \mathrm{~m}$. Because Brazilian reserves are characterized by their location in relation to the salt deposits, reserves placed above the salt layer are called Post-salt, and those below the salt layer are called Pre-salt. For this reason, Petrobras is the second operator with the largest number of FPSO units (about 12 owned and 14 operating), utilizing over $15 \%$ FPSOs of all those existing worldwide (SHIMAMURA, 
Figure 1.1 - Photo of the typical FPSO on site

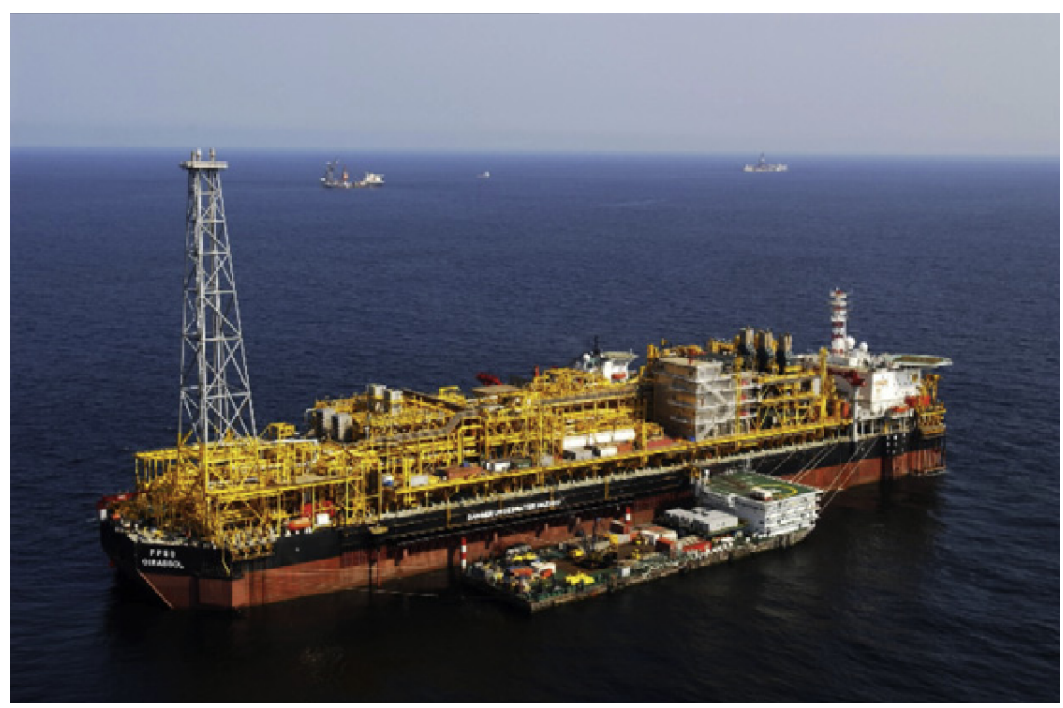

Source: (FONTAINE et al., 2013)

\section{2)(HALLIBURTON, 2014)(BARRERA et al., 2015).}

The concept of FPSO is for working as a floating unit, which can be used for the primary production of petroleum and gas. This allows storing the explored petroleum in a repository tank, besides being able to offload to another storage unit. A typical FPSO is described briefly in the next subsection.

\subsubsection{Primary Separation Train of Petroleum}

In a primary processing installation, the role of the processing plant is to separate the well fluid into three components. Thus, the crude oil comes into the separation train, which consists of several stages and separator types. For example, in a three-phase separator known as gravitational separator (Figure 1.2), Gas as a less dense fluid, is initially separated from liquids by the action of gravity and water with more density separates under oil.

The separated gas in the separator train is forwarded to the compression units of the platform, and water is sent to the produced water treatment system. Next, the processed oil goes through two sequences of heat exchangers, to raise its temperature to levels that facilitate separation in the subsequent part. The hot fluid of the first sequence of heat exchangers is the oil stream (as exportation oil) leaving the processing plant to the Cargo Tank and the hot fluid of the second heat exchanger is provided by the hot water from the Waste Heat Recovery Unit of gas turbines (MORAIS, 2013).

In the next steps of separation, there are two similar pairs of heat exchangers, called Degassers and Electrostatic treaters. Degassers are responsible for separating of the light 
Figure 1.2 - Gravitational Separator

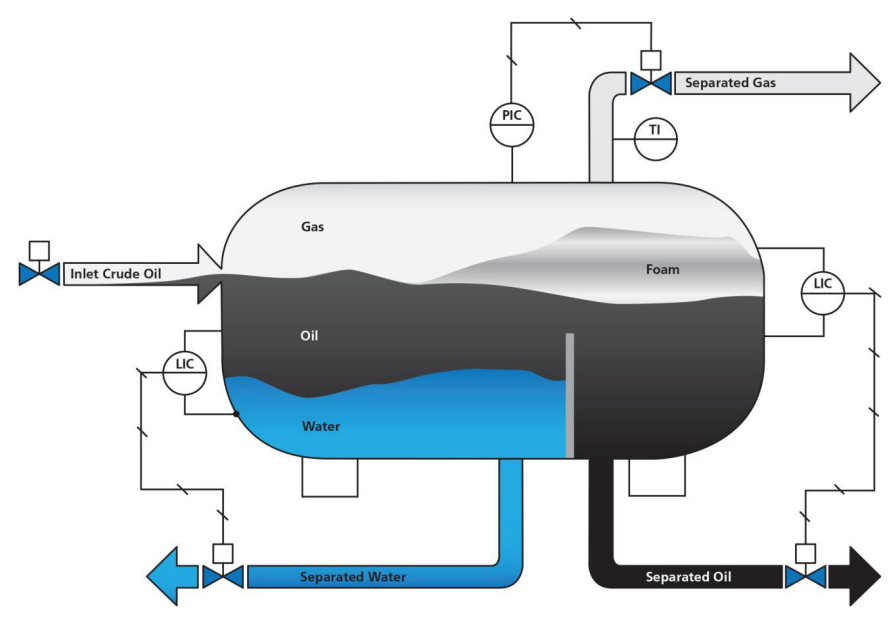

Source: (PROCESSONLINE.COM.AU, 2014)

hydrocarbon fractions in operating pressure of about eight bars. The output oil of that separator is forwarded to the Electrostatic treater. In that, water drops remain separate from the oil by electric polarized plates with the alternative current. The second pair of heat exchangers operates in the same way, but the pressure level is lower, in order to have an increment separation before transmitting oil to the cargo tanks (PETROBRAS, 2007).

\subsubsection{Gas compression treatment, re-injection and exportation system}

The phase of each treatment process is designed to achieve the necessary criteria to enable its appropriate destination. For gas, the targets are forwarding, exporting via pipeline and sometimes re-injecting them in a reservoir. Therefore, reducing the number of contaminants to acceptable levels, and achieving the proper initial pressure are important points.

After separation processes, there are three gas streams with different pressures; high, medium and low-pressure levels. High-pressure gas that comes from the main separator (gravitational separator) is forwarded directly to the main gas compression unit. Medium and low-pressure gases must go through an additional system, called Vapor Recovery Unit (VRU) to recover and to complete its pressure to the suction level of the main compressors. In the main compression unit, after the input gas goes through a scrubber vessel, there are three compressors and three gas-water coolers to remove the thermal load absorbed by the gas during compression.

The received gas with a low content of $\mathrm{CO}_{2}$, after $\mathrm{CO}_{2}$ removal, is sent to the compression system of exportation. There, the pressure of the gas stream is elevated up to about 250 bar, which is required from the pressure level for the pipeline to transport the gas (MORAIS, 2013). 


\subsection{3 $\mathrm{CO}_{2}$ Removal, Compression and Injection System}

The gas without water and heavy components enters the $\mathrm{CO}_{2}$ Removal system composed of membranes. The input gas has $\mathrm{CO}_{2}$ content ranging from $8-40 \%$ and after going through the membranes, one output that has $\mathrm{CO}_{2}$ content in its composition varies between $2-5 \%$ and another output varies between $30-50 \%$. This gas stream with greater $\mathrm{CO}_{2}$ content is routed to the $\mathrm{CO}_{2}$ compression unit, where its pressure is raised to a level of 250 bar (the initial feed pressure of re-injection compressors) and then it is re-injected with the pressure of 500 bar (MORAIS, 2013).

\subsection{FPSO operational modes}

Operating conditions are often determined by the features of the fluid reservoir, based on the composition of the hydrocarbons and on the amount of impurities in the oil content. According to the crude oil composition of pre-salt wells, the operating life of a reservoir fluid and consequently, the operational modes of FPSO are divided into three general modes: Mode 1, 2 and 3.

\subsubsection{Operational mode 1}

Operational mode 1 represents the typical early life condition and is applied when the crude oil has a high GOR (gas-oil ratio ) and all of the processed gas is assumed to be exported and the removed $\mathrm{CO}_{2}$ is injected into the wells. In this operational mode, the fuel gas is obtained from the treated gas after the $\mathrm{CO}_{2}$ membrane unit.

\subsubsection{Operational mode 2}

This operational mode is used when the crude oil contains $50 \%$ BSW. In operational mode 2 , $50 \%$ of the separated gas from the separation processes is injected in the $\mathrm{CO}_{2}$ removal unit in order to be exported and $50 \%$ of the bypassed gas is injected into the production wells at a pressure of 494 bar, approximately.

\subsubsection{Operational mode 3}

Operation Mode 3 is the end of life condition of an oil field and in that, all the gas separated from the crude oil with the maximum quantity of water/ $/ \mathrm{CO}_{2}$, is injected into production wells through a bypass located in the $\mathrm{CO}_{2}$ removal system. 


\subsection{Motivation}

A Floating Production Storage and Offloading (FPSO) plant is a high energy consumer (from a few to several hundreds of megawatts). The fuel consumption, power demand, and production of a typical FPSO change regarding the operating conditions and lifetime of a field. The possibility of improving for a FPSO plant configuration (in current operation) from early life to the end of life of reservoir by changing thermodynamic operating parameters through a formal optimization procedure has motivated the development of the current thesis. Moreover, applying a systematic and automation optimization procedure to increase the sustainability and profitability of a FPSO simultaneously, without adding any new technology and imposed costs, is necessary to address existing gaps. Finally, suggesting a new standardized design from the optimization configuration, for a Brazilian FPSO that meets the technical challenges related to pre-salt oil field and operating in offshore conditions, is considered in the objectives framework of the current research.

\subsection{Objectives}

The main objective of this thesis is the development and application of an optimization methodology, based on the thermodynamics analysis and sensitivity analysis, for proposing optimum and sustainable configurations of Primary Petroleum Platform of typical FPSO. Or rather:

- Implementation of thermodynamics analysis to find important operating parameters on energy consumption sources for the existing configuration of main and utility plants in a FPSO Primary Petroleum Processing using the real performance data of applied gas turbine;

- Application of a screening analysis to identify the main and interaction effects of thermodynamic parameters on fuel consumption, hydrocarbon liquids recovery and performance of separation (as one of the possible improvements) for specific scenarios related to a Brazilian FPSO operating on a pre-salt oil field;

- Application of an appropriate optimization procedure for fuel consumption minimization and maximization of hydrocarbon liquids stabilization and recovery as a step in the improvement of separation performance purposes, subject to several constraints, of a Brazilian FPSO for early life, mid-life and end of life of a pre-salt oil field.

- Integrating of Aspen Hysys as a robust simulator of chemical processes and ModeForntier as an automation process of screen analyzing and optimization procedure to achieve the presented item above. 


\subsection{Outline of the Thesis}

The thesis is divided into six chapters.

Chapter 1 introduces a brief outlook on the importance of the oil and gas industry in the global Energy Matrix, indicating the current and ahead energy and environmental challenges of this industry, along with the motivation, objectives, and outline of this thesis.

Chapter 2 sets the literature review of the offshore industry, including the role of crude oil type on processing and utility plants, Brazilian offshore and FPSO oil and gas industry, and thermodynamics analysis of these plants. Furthermore, this capture contains a brief revision of the system modelling methods, screening analysis, optimization procedures, and application of process optimization into oil and gas processing plants;

Chapter 3 describes the theoretical foundations and methodologies for determining major energy consumers, indicators of separation performance. Moreover, algorithmic optimization methods, statistical analysis methods, and focusing on Genetic algorithm techniques are presented in this chapter;

Chapter 4 shows the description and implementation of the modeling and simulation of the FPSO plants considering three operational modes and well-fluid compositions in its useful life. Additionally, the strategy of an integration of simulation and optimizer to perform automated sensitivity analysis and optimization is explained;

Chapter 5 demonstrates the obtained results from modeling, sensitivity analysis, and optimization procedures that meet the desired objectives of the current thesis.

Chapter 6 concludes the present thesis, summarize the main findings of this work and pinpoints the possibilities for future ones.

Figure 1.3 shows the generic steps to achieve the results of the current thesis. 
Figure 1.3-Outline

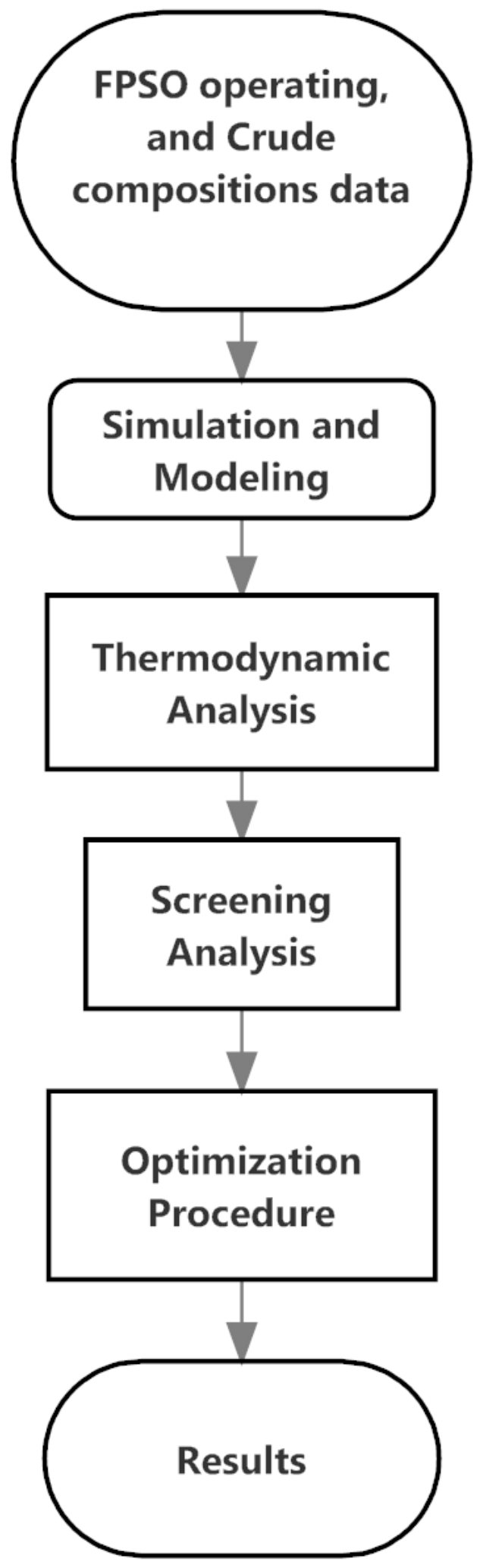





\section{LITERATURE REVIEW}

The last decades have witnessed the development and application of energy efficiency tools to various thermal systems and industrial applications. Several authors have studied thermodynamics, economic and environmental analysis of the oil and gas production base platforms in diverse operating condition ranges, reservoir fluids, processes and technologies. However, the oil and gas processing platforms are energy-intensive systems and many fulfilled works using thermodynamics analysis confirmed that, but, there are very few researches, which discussing the possibility of improving of a plant configuration (in current operation) through an optimization procedure in an offshore.

This chapter provides an overview of the most relevant research works, to discuss the state of art in the literature. The studies are divided into two main subjects, which are considered for this chapter content.

\subsection{Primary Petroleum and Gas Processing Offshore Platform}

To analyze a typical offshore, understanding relation among components and structure is essential. Thus, this section presents the generalized information of reservoir fluid and processing platforms in two first sub-section and eventually, a Brazilian standardized FPSO as the studied case is described.

\subsubsection{Composition, Crude Oil, Gas and Reservoir Fluid}

The main function of an offshore platform is to separate oil from reservoir fluid and associated gas. Reservoir fluid is a complex mixture contained within the hydrocarbons and a wide variety of other solution and chemical components. It is in liquid form at condition of underground reservoirs and remains a liquid when brought to the surface. The composition and properties of each well differ significantly from one reservoir to another. Petroleum derivations of the wells are produced from processing crude oil and other liquids, such as high-content heavy hydrocarbons, intermediate and volatile hydrocarbons, methane, light hydrocarbons and water at petroleum processing platforms.

The hydrocarbon in crude oil compounds belongs to one of the following subclasses (IUPAC, 1993)(ABDEL-AAL et al., 2015):

- Alkanes or paraffins which are saturated hydrocarbons with the general formula $\left(C_{n} H_{2 n+2}\right)$. They may be straight-chain or components in branched form, because of the production of high-octane gasoline, the latter are more valuable than the former; 
- Cycloalkanes or cycloparaffins (naphthenes) which are unsaturated hydrocarbons (examples are cyclopentane $\left(C_{5} H_{10}\right)$ and cyclohexane $\left.\left(C_{6} H_{12}\right)\right)$. The presence of large amounts of these cyclic compounds in the naphtha range is significant in the production of aromatic compounds;

- Aromatic hydrocarbons that only monomolecular component in the range of $C 6-C 8$ have gained commercial importance.

According to McCain et al. (2011), a reservoir fluid regarding some thermodynamic properties, such as pressure and temperature, and composition can be categorized into following main classifications:

- Dry Gas: All hydrocarbon components are in the gas phase in the reservoir or at the surface as shown in Figure 2.1. The word "dry" mentions that this gas hydrocarbons does not contain enough of the heavier components to form hydrocarbon liquid at the surface.

Dry Gas is basically methane with some intermediate. The pressure path line $(1 \rightarrow 2)$ does not pass from the phase envelope at reservoir and surface separator conditions and consequently, there is only dry gas. Point 1 presents in the reservoir condition and point 2 is at the surface (condition). Thus, theoretically, no hydrocarbon liquid is formed at the surface;

Figure 2.1 - Phase diagram of a typical dry gas reservoir with a line of reduction of reservoir pressure and surface conditions.

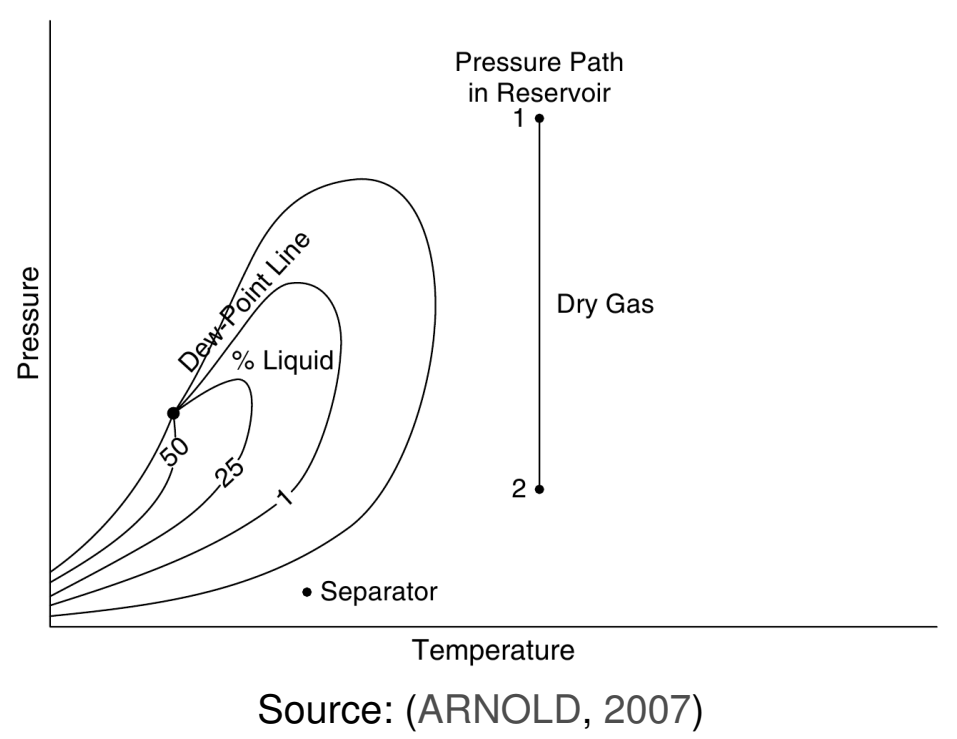

- Wet Gas: The majority of hydrocarbons are present in the gas phase in the reservoir and at the surface. However, a small fraction release as condensate at the offshore processing conditions (Figure 2.2). In fact, the reservoir fluid is normally saturated with water and the word "wet" does not mean that the gas is wet with water. But it refers to the hydrocarbon liquid, which in some condition at surface, gas can be condensate at surface conditions (ARNOLD, 2007). Wet Gas contains heavy components of intermediate hydrocarbons, such 
as propane and butane. However, some liquids tend to be formed in separation condition at the surface, and this liquid is normally called condensate. As it is shown in Figure 2.2, the pressure path does not enter the phase envelope, and thus no liquid is formed in the reservoir. Separator conditions lie within the phase envelope, causing some hydrocarbon liquid to be formed at the surface;

Figure 2.2 - Phase diagram of a typical wet gas reservoir with a line of reduction of reservoir pressure and surface conditions.

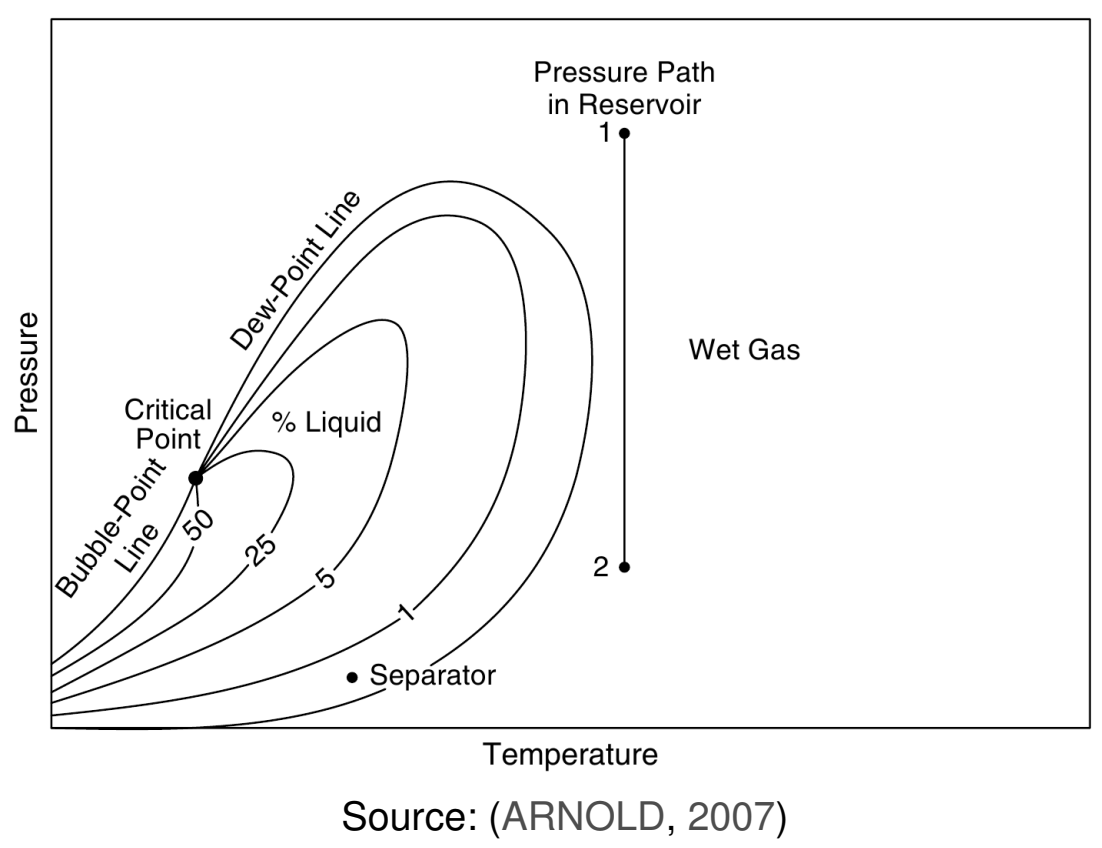

- Gas Condensate: The Gas Condensate reservoir is also known as a retrograde gas condensate reservoir. At the beginning, the condensate gas is totally gaseous in the reservoir (point 1 in Figure 2.3). Condensate Gas has a temperature (and pressure) more than the critical temperature (and pressure) of the fluid at reservoir condition that leads it to be in gas form. As Figure 2.3 shows, when the pressure reduces in the reservoir, the fluid enters in dew point and a large volume of liquid begins to condensate in the reservoir (Point 2). With further depleting and increasing the pressure drop, liquid condenses form as the free liquid (Point 3).

Gas condensate reservoirs proved an initial producing gas-oil ratio (GOR) between 3300 and 150,000 SCF/STB. With decreasing the pressure below the dew-point line (in two phase area), the production of gas condensate in gas-oil ratios will begin. However, according to type of high carbon components such as $\mathrm{C} 20+$, the position of critical point of condensate and wet gas can totally changes (KIM et al., 2014). The surface gas is very rich in intermediates and often is processed to remove liquid propane, butanes, pentanes, and heavier hydrocarbons. These liquids are called plant liquids (ARNOLD, 2007); 
Figure 2.3 - Phase diagram of a Condensate gas reservoir with a line of reduction of reservoir pressure and surface separation conditions.

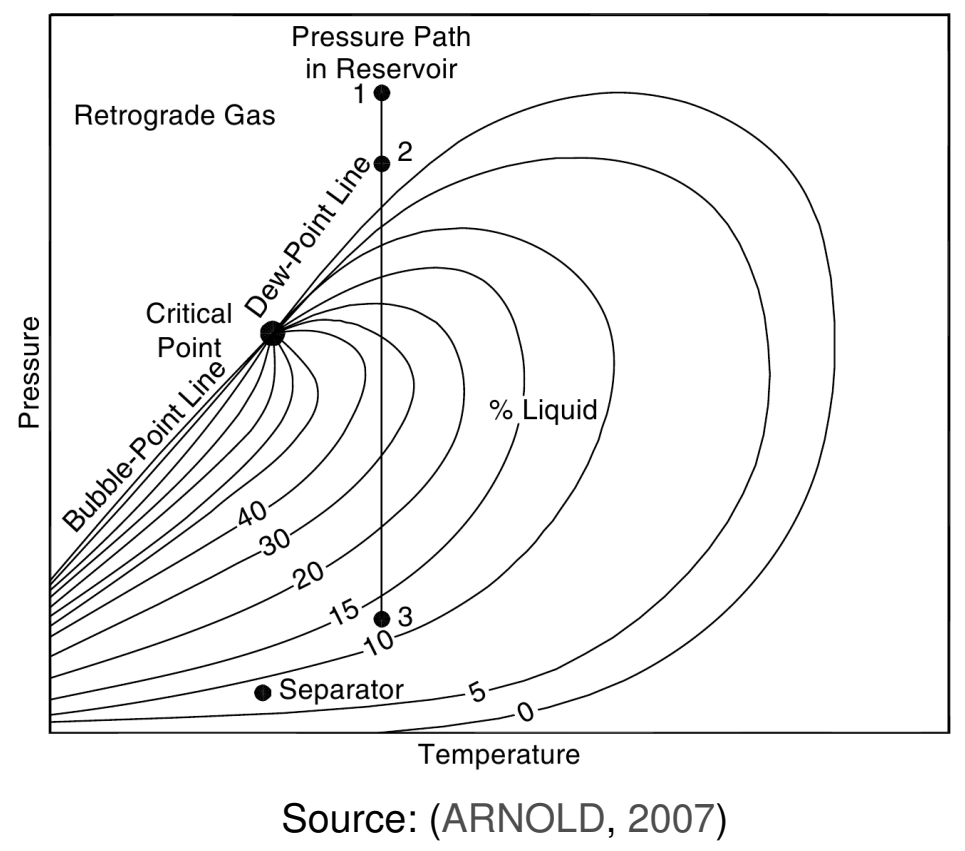

- Volatile Oil: It is rich of heavy components and more intermediate hydrocarbons than Black oil reservoirs. volatile oil mixed with associated gas is very similar to Condensate Gas with the difference that the reservoir temperature of volatile oil is lower than its critical temperature (Figure 2.4). Therefore, volatile oil can flash to more gas content with a small reduction in pressure below the bubble point;

Figure 2.4 - Phase diagram of a volatile oil reservoir with a line of reduction of reservoir pressure and surface separation conditions.

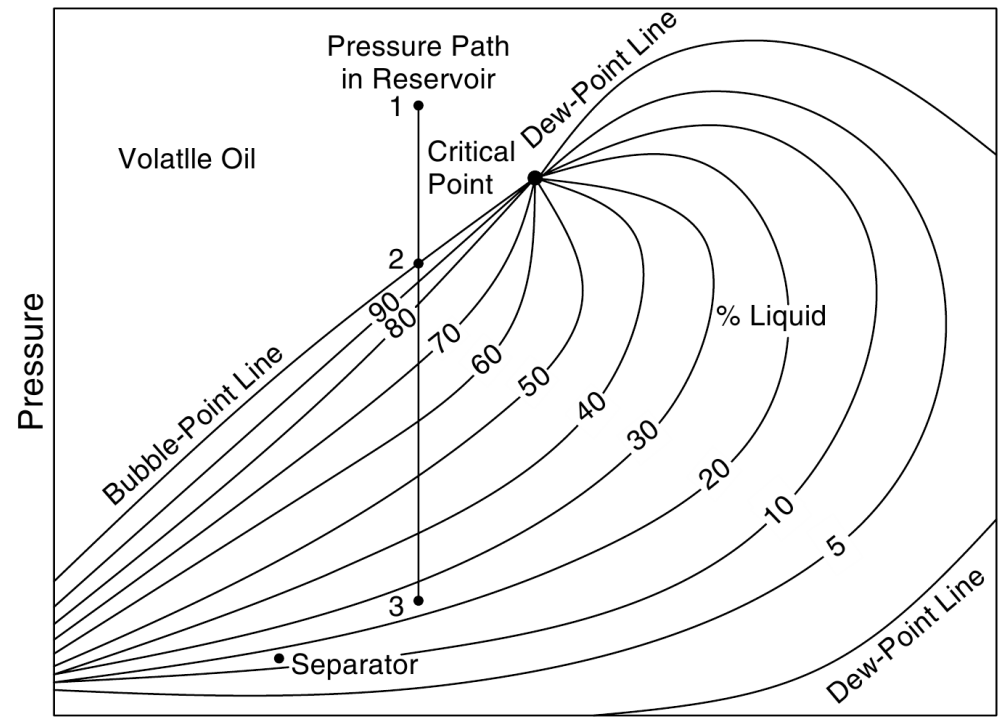

Temperature

Source: (ARNOLD, 2007) 
- Black Oil: Mostly large, heavy, and non-volatile hydrocarbons. When the reservoir pressure downs along line $1 \rightarrow 2$, the oil gets undersaturated and if presents more gas, it does dissolve more gas. No gas forms in the reservoir until the pressure reaches the bubble point, at which point it gets saturated and contains as much dissolved gas as it can hold (ARNOLD, 2007). At point 2, the oil is with the maximum content of the dissolved gas (Figure 2.5). Separator conditions lie well inside of the phase envelope, stating that a relatively large amount of liquid arrives at the surface (Point 3). The laboratory determined composition of heptane plus will be higher than 30 mole percent, thus indicating a large quantity of heavy hydrocarbons in black oils (ARNOLD, 2007).

Figure 2.5 - Phase diagram of a black oil reservoir with a line of reduction of reservoir pressure and surface separation conditions.

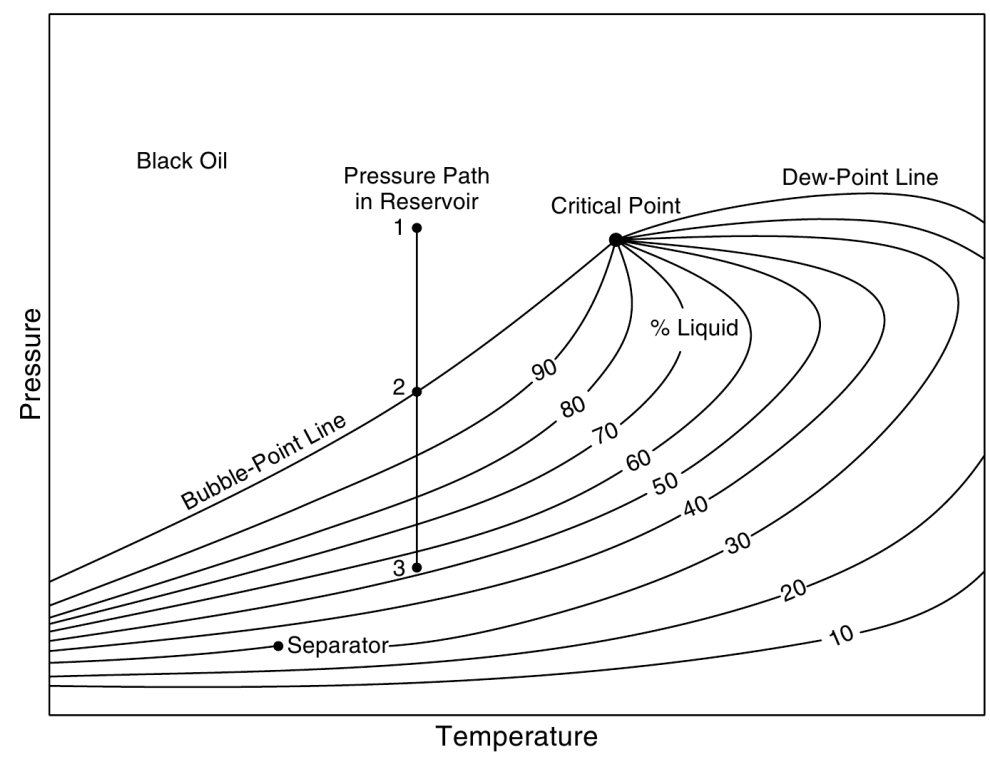

Source: (ARNOLD, 2007)

\subsubsection{Offshore Platforms: Processes and Configurations}

The present subsection reviews several configurations of main oil and gas processing plants in typical offshore platforms to address existing models that are similar to proposed FPSO (sections 1.2 and 4.1). Thus, the generic configurations of offshore plants in literature are explained and finally, the differences of them with FPSO platforms are discussed in order to understand the necessary processes.

Today's, the role of offshore plants in oil and gas industry is irrefutable, so that the offshore production is nearly $30 \%$ of global crude oil (and gas) production (U.S. ENERGY INFORMATION ADMINISTRATION, 2016). Therefore, oil and gas companies have been decided to increase the implementation of offshore in a more sustainable and efficient way to meet their 
operations and desired productions in short-term and long-term plans.

The primary design of an offshore is based on: first, the oceanic conditions such as site temperature and second, well conditions such as well composition, useful years of operation, distance from cost and depth from sea level. Note that the crude oil composition has a decisive role in the configuration of the main and utility systems of an offshore plant (ETA-OFFSHORE-SEMINARS, 1976).

Arnold \& Stewart (1998) in their book with title ofdesign of oil-handling systems and facilities explained that a typical offshore platform consists of several main plants where separation, compression, treatment and pumping processes are carried out, and utility plants are considered to provide the required power and heating for the main plants. Arnold \& Stewart (1998) also indicated that the performance of processing plant may be impacted by many key parameters, such as well-fluid flow rates, operating pressures and temperatures, well fluid properties, the final treatment of productions, among others.

BP (2004) in the third phase of Azari, Chirag \& Gunashli after finishing the Full Field Development about main plants of the petroleum platform reported that separation train is the most important unit for the oil and production. However, (BP, 2004) did not mention the details of the used crude oil composition and operating pressure of sequential separators, but there is some useful information from the general scheme of his report.

As shown in Figure 2.6, the production manifolds are responsible to receive reservoir fluid and to decrease pressure of crude oil before entering into separation train. The separation train consists of three and two-phase separators and coalescers that they are operating at different pressures depending on the conditions of the perforations in reservoir. Most of the associated gas from the reservoir fluid is obtained in the high pressure separator and the separated oil is processed in a coalescer to reduce the water content. Then, the produced oil is conveyed to the main line of oil pumping for exportation. The produced gas should be prepared before routing to other units. Thus, the separated gas is led to the gas treatment units where it is cooled and dehydrated to be compressed and exported. Gas can also be treated and then injected into the reservoir in order to enhance oil recovery (EOR). BP (2004) concluded that regarding the working conditions (conditions of labor) on the platform, temperatures and the pressures of the fluid reservoir, GOR (gas-to-oil ratio) and WOR (water-to-oil ratio), petroleum properties, oil recovery, and other additional considerations, there are different petroleum plant process configurations. 
Figure 2.6 - Offshore Production Process

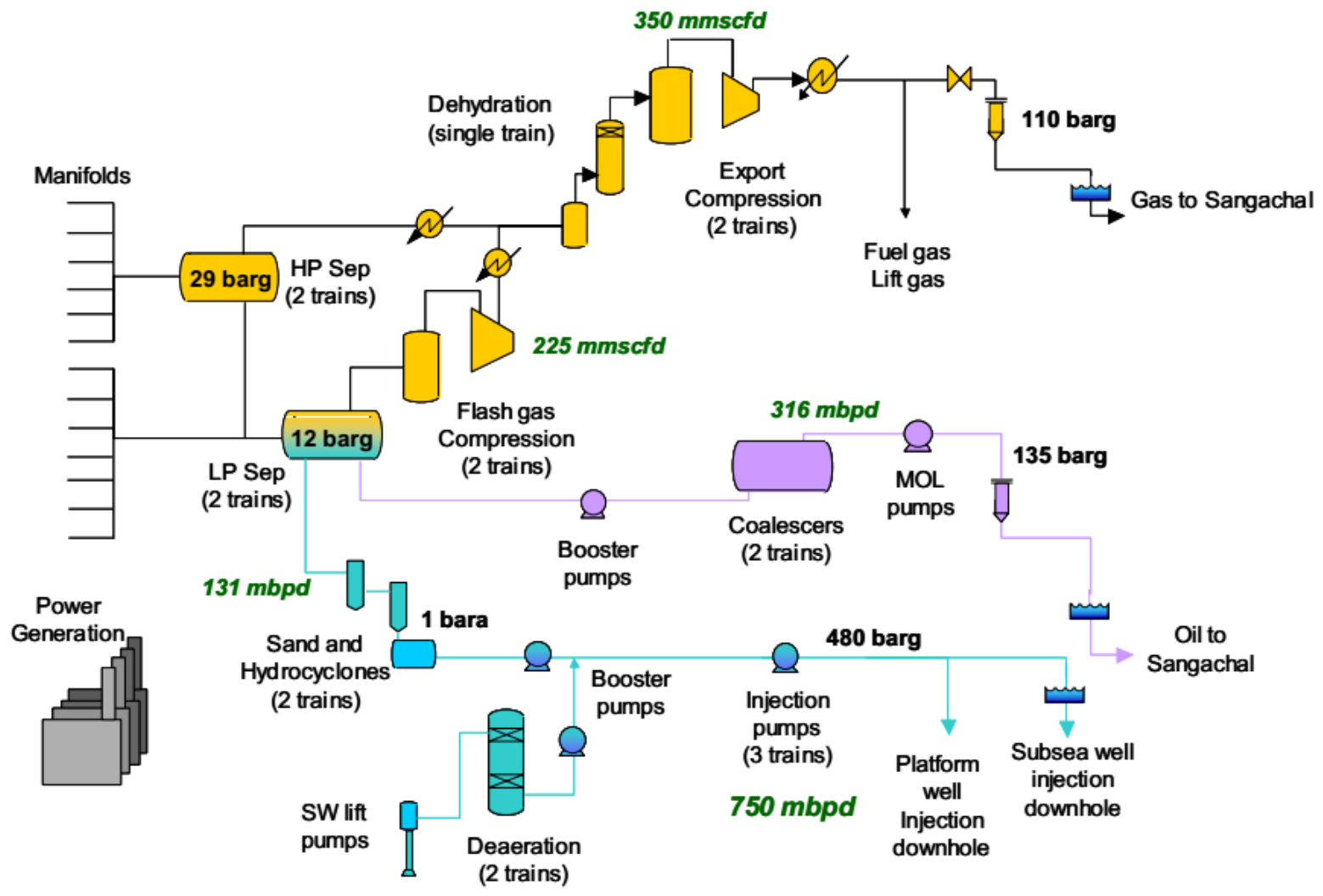

Source: (BP, 2004)

Devold (2006) published a detailed diagram of the main processes in the oil platform including chemical products and control instruments in Norsk Hydro Nyord that is a more completed work compared to BP (2004). He also indicated some details in configuration to ensure the quality of the separation products.

In a Brazilian reservoir case, Beltrao et al. (2009) explained that for a pre-salt crude oil composition with a considerable content of $\mathrm{CO}_{2}$, besides of $\mathrm{CO}_{2}$ separation from gas, it is necessary to design an injection unit of $\mathrm{CO}_{2}$. Then, they indicated that adding new required units is not an easy task in the limited space and condition of an offshore.

Nguyen et al. (2013) presented a division of the petroleum separation processing plant for a Norwegian offshore plant and then, explained the different units and processes of an offshore. The following items are some main and utility plants of a typical offshore:

- Transference of the reservoir fluid through pipes and production manifold;

- Depressurization of fluid in the strangler boxes;

- Separation of liquid and gas in the separation equipment; 
- Pumping systems to export oil, storage and final treatment to pump onshore;

- Treatment of produced gas in separation to remove water from it;

- Compression of produced gas to export it, to use in gas-lift or electricity generation unit(s);

- Treatment of the produced water in the separation to be returned to the sea or other purposes in the plant.

\subsubsection{Brazilian Reservoir, Offshore industry and FPSO Configurations}

Exploration of the discovered reservoir in 2007, confirmed a significant potential to develop petroleum resources in Brazil, especially the pre-salt areas. The pre-salt area is characterized by deepwater and ultra-deepwater oil field with water depth around $2200 \mathrm{~m}$ and a layer of salt that reaches about $2000 \mathrm{~m}$ in thickness (FORMIGLI, 2007). As shown in Figure 2.7, Brazil was the second-largest offshore producer in 2015 and by supporting small production, this increment continues in 2016 and 2017 (U.S. ENERGY INFORMATION ADMINISTRATION, 2016). Likewise, the recently published report of Ministério de Minas e Energia in 2017 confirmed the prevision of EIA and showed an increase of $3.2 \%$ compared to 2015 that it means an additional produced oil of 81 thousand barrels per day (Ministério de Minas e Energia, 2017). However, there is an increase of $7.9 \%$ (+7.6 million cubic meters per day) in natural gas production that should be considered as important as in oil production strategies.

Figure 2.7 - Global offshore crude oil production, 2005-15.

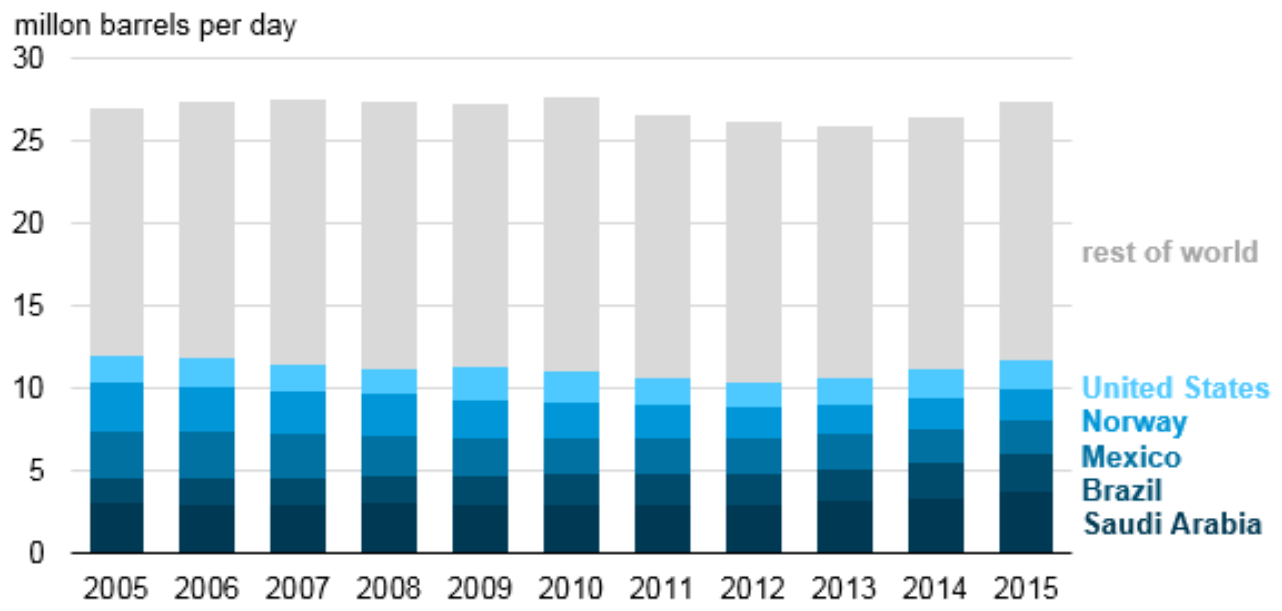

Source: (U.S. ENERGY INFORMATION ADMINISTRATION, 2016) 
The pre-salt areas are located in water depth ranging from 2,000 to 2,500m, spread over very large areas, around $300 \mathrm{~km}$ from the coast. Given these points, FPSOs can be the first option for pre-salt areas, mainly due to crude oil and natural gas storage capabilities. Thus, does not require the construction of long-length oil pipelines, and also because of other characteristics that allow a short-term completion with economic advantages. On the other word, FPSO plant is relocatable to other fields, its value retains and upfront investment and abandonment costs are less than fixed platforms. Therefore, FPSO structure and its development were chosen by Petrobras and partners for extraction, processing and exportation of oil and gas (Minerals Management Service Gulf of Mexico OCS Region, 2000) (BELTRAO et al., 2009)(ANDRADE et al., 2015).

Hence, Brazil is turned to use the FPSO facilities extensively as from 2009 to present. Only until 2013, US $\$ 174.4$ billion were investigated for pre-salt area in order to increase Brazilian oil production that allowed Brazil to have more than 63 new vessels and offshore platform. However, those investments have been affected in 2014, because of economic crises. Nevertheless, the production of liquid fuels in Brazil has increased due to the operation of five new FPSOs: P-62, P-58, P-57, Cidade de Ilhabela and Cidade de Mangaritiba (OFFSHORE CENTER DENMARK, 2009)(U.S. ENERGY INFORMATION ADMINISTRATION, 2015b).

A typical FPSO has almost the same main and utility plants of a fixed offshore. In that there are oil and gas processing and treatment, and the oil and water require for specific treatment operations to achieve the expected specifications of the productions. Gas is scrubbed, dehydrated and compressed in order to be used as fuel, to be injected as GOR and/or to be exported. Moreover, produced oil is separated from water, gas, etc., by the separation processes, and then the treated oil may be stored, transferred or exported. Separated water is prepared to remove the emulsified oil before disposal at sea. Hence, all oil and gas production operations are projected in a very complex and well-structured processing plant to attend different operating and demands of the production process (MUELLER; ROOBAERT, 2008). Therefore, due to increasing the benefit of a large machine such as FPSO, standardization is necessary. Because it can reduce risks of delay and poor performance in the equipment design and supply (PINTO et al., 2014).

Figure 2.8 shows a scheme of the basic production separation and treatment unit of a standardized FPSO unit. In that, subsea crude oil from the production manifold enters in the basic separation train, which has two separation stages: high and low pressure. Separated gas is routed to treatment processes (compression, dehydration and dew point control) in order to be injected or exported. Produced oil is sent to a set of more treatments such as oil stabilization that ensuring the final specifications. Separated water from separators is prepared to be discharged into seawater. Additionally, seawater can be injected into the wells. The optional units are shown as necessary for project-specific production requirements. 
Figure 2.8 - Standardized FPSO processing scheme includes basic production separation and treating systems.

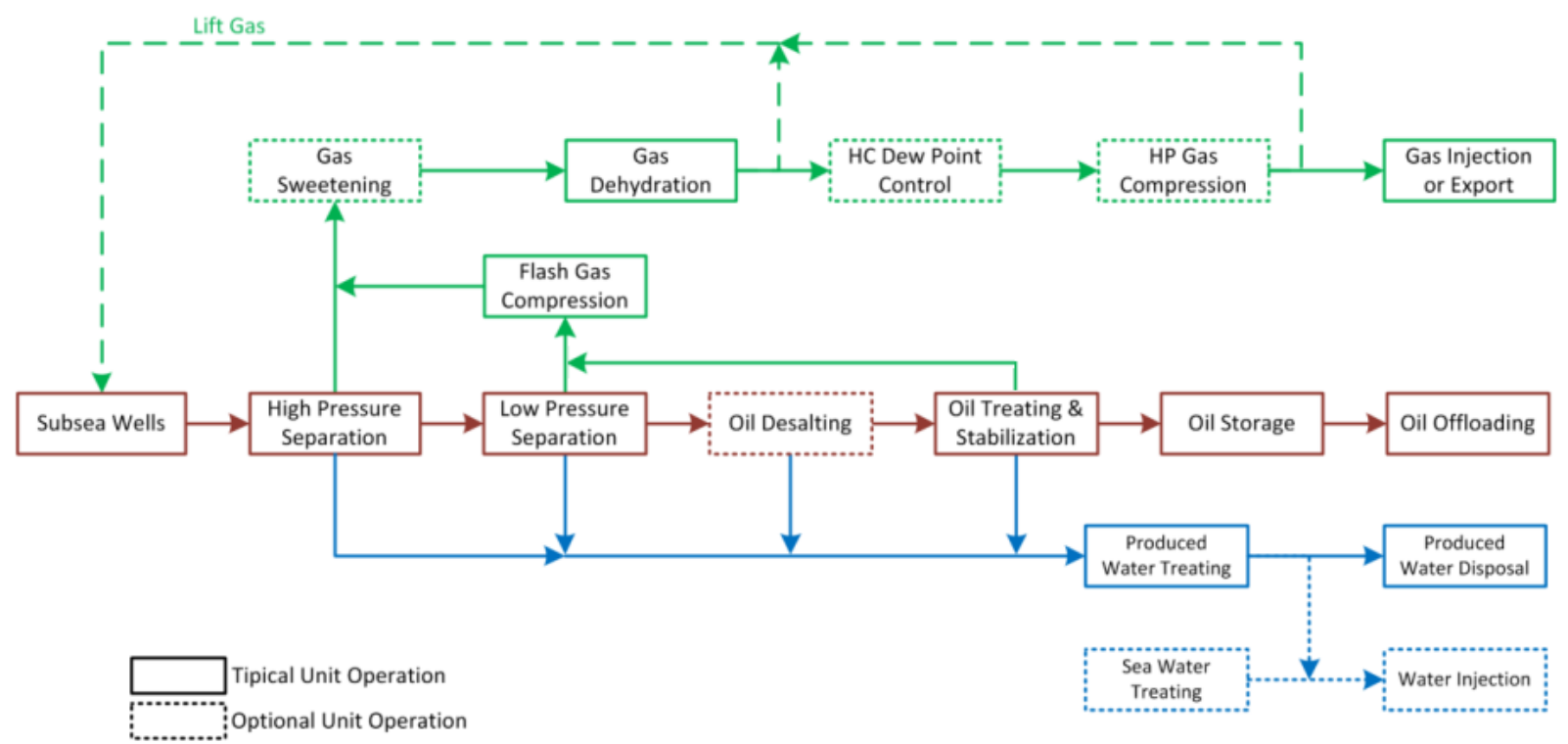

Source: adapted from (MUELLER; ROOBAERT, 2008)\&(SÁNCHEZ, 2017)

The FPSO plant size and design, required processing plants and consequently, the oil capacity, depend on the following parameters (PINTO et al., 2014):

- Amount of gas solubilized in the oil or GOR;

- $\mathrm{CO}_{2}$ content in produced gas;

- Need for $\mathrm{CO}_{2}$ removal;

- Water injection flow rate in order to pressure maintenance;

- Oil treatment difficulty.

For instance, the standard FPSOs are supposed to be used in Santos Basin pre-salt area, regarding crude oil composition, the presence of fluid contamination such as $\mathrm{CO}_{2}$ and $\mathrm{H}_{2} \mathrm{~S}$, GOR and area of well positions, besides what was discussed above, must have a nominal capacity of processing $150,000 \mathrm{bpd}$ of oil, $100,000 \mathrm{bpd}$ of produced water and $6,000,000$ $\mathrm{Sm}^{3} / \mathrm{d}$ of gas. In addition, concerning the gas plant, the main concept of FPSO consists in the following steps (ANDRADE et al., 2015):

- A molecular sieve for gas dehydration unit;

- A hydrocarbon dew point control unit;

- A single stage of $\mathrm{CO}_{2}$ removal membrane with maximum $3 \%$ of $\mathrm{CO}_{2}$ content in exported gas. 
Moreover, an electric power unit is designed to provide the demand of $75 \mathrm{MW}$ as utility plant. A simplified overall scheme of the Standards FPSO process facilities with its operating pressure and temperature is presented in Figure 2.9. As can be seen, there are four processes plant: a separation train with three operating pressures and temperatures (Pre-heater and heater); gas treatment and compression with vapor recovery unit; $\mathrm{CO}_{2}$ removal and; compression and water treatment.

Figure 2.9 - Overall scheme of a standards FPSOs topside process facilities.

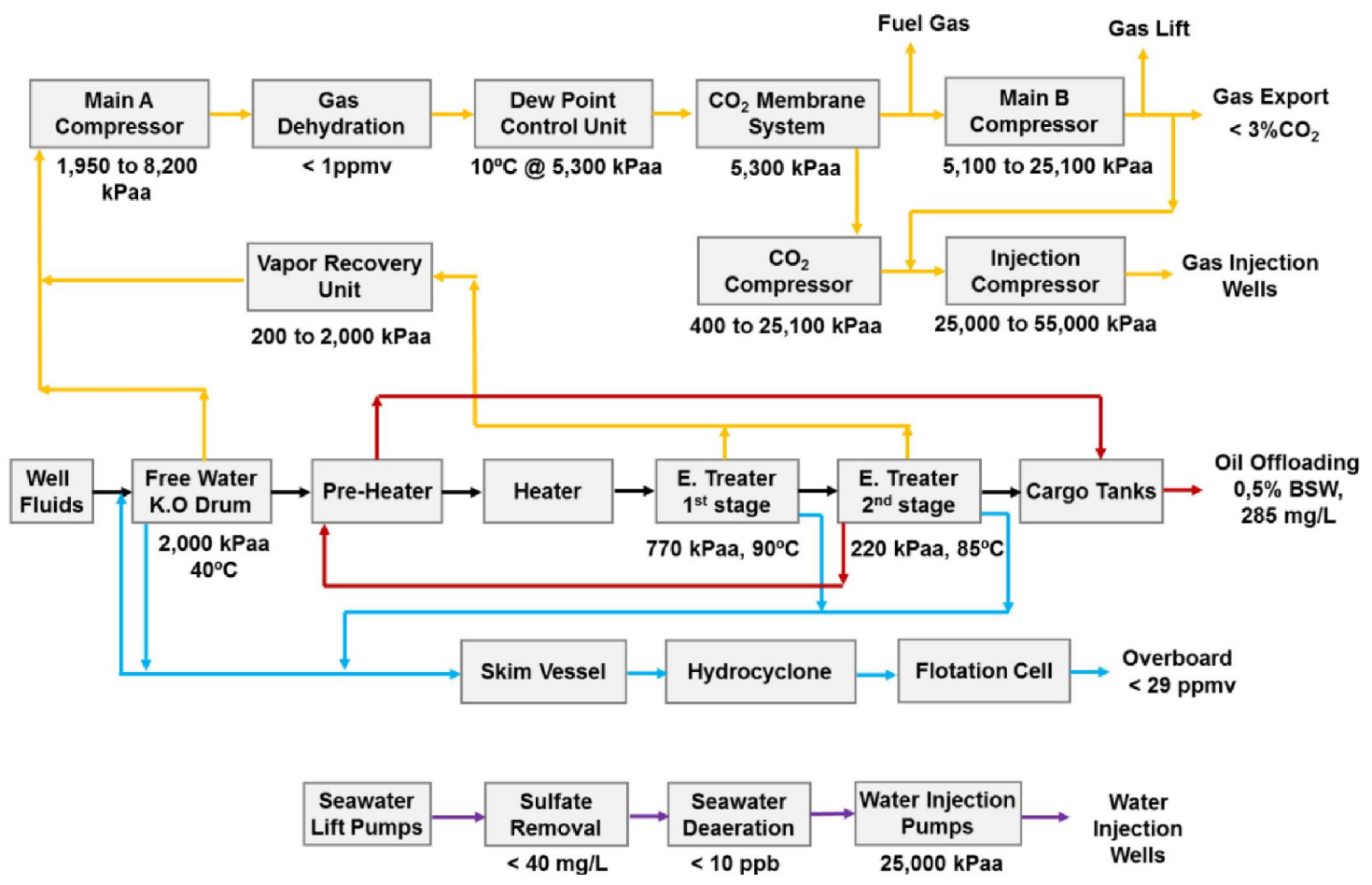

Source: (ANDRADE et al., 2015)

Although, the approach for connecting the pre-salt reservoirs to existing infrastructures, or existing units retrofitting were evaluated. In some cases, those strategies cannot provide by existing FPSO to address the requested capacities. Therefore, new units are designed and called "Replicant." The replicant units are constructed specifically to meet all that discussed above for a standard FPSO. Hence, the discovery of the pre-salt gave Petrobras the opportunity to extend the usage of this standard concept and to contract eight new units (P-66, P-67, P-68, P-69, P-70, P-71, P-72, P-73) (PINTO et al., 2014)(ANDRADE et al., 2015)(NUNES et al., 2016).

Figure 2.10 shows a Replicant FPSO module, which is 22 kton of topside dry weight. There are 17 processing plants for an oil production of $150 \mathrm{kbpd}$ including $\mathrm{CO}_{2}$ removal unit. 
Figure 2.10 - Modules of a Replicant FPSO.

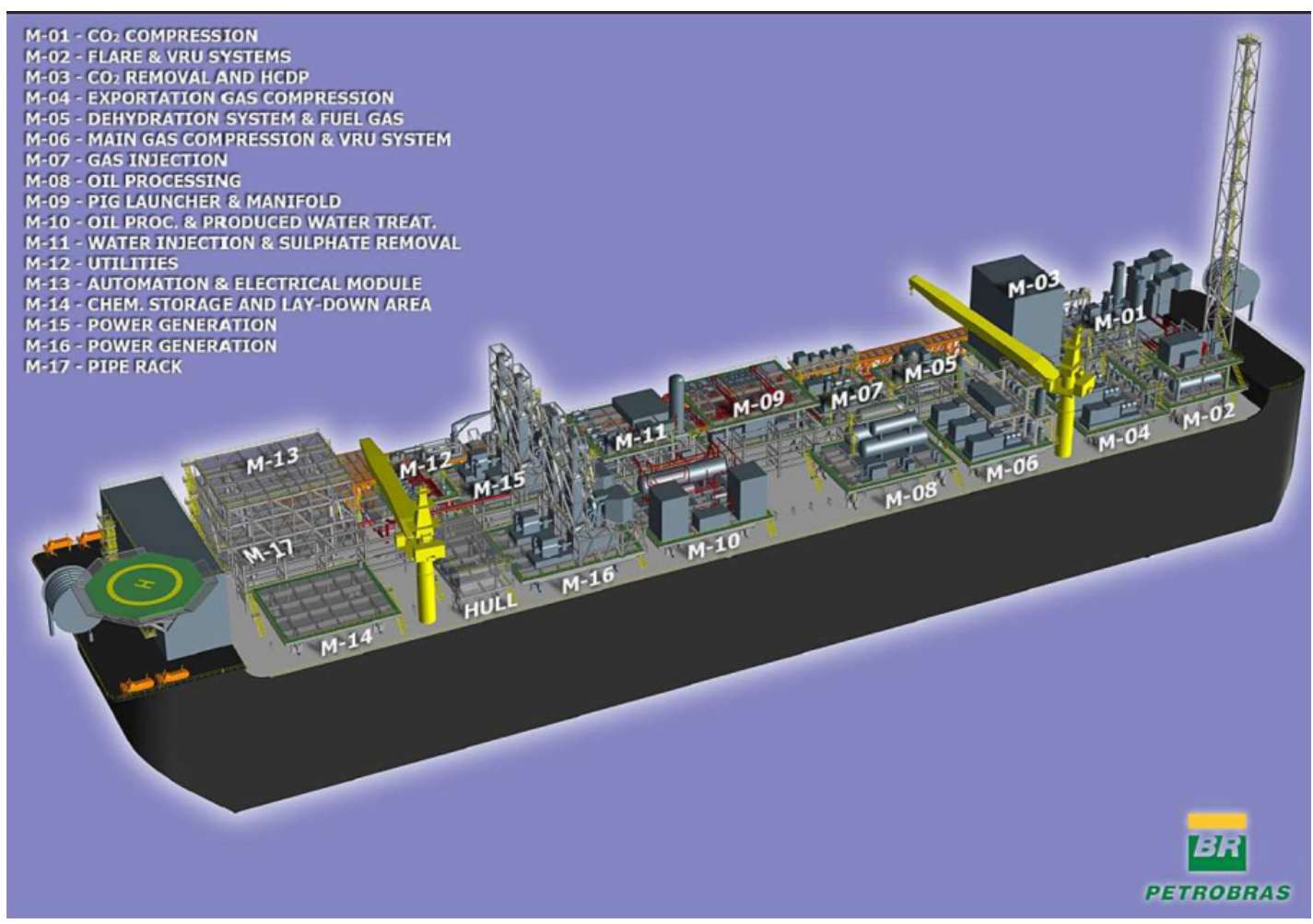

Source: (NUNES et al., 2016)

\subsubsection{Thermodynamics analysis of oil and gas processing plant}

In the last decades, several tools based on the First and Second Laws of thermodynamics have been developed for defining indicators to assess the performance of chemical and industrial processes including oil and gas processing plant. This subsection lists several evaluation of performance articles in oil and gas processing platforms. Energy is the first indicator approached in most of the papers on performance evaluation of various industrial processes. Although, other concepts, such as environmental impact and exergy are also presented.

Manning \& Thompson (1995) as one of the primary researches, performed an energy analysis to indicate the major users and consumers in an oil field processing with three-stage separation train.

The first work on the thermodynamic performance of oil and gas offshore processing was the exergy analysis by Oliveira-Jr. \& Hombeeck (1997). They analyzed a Brazilian case facility, where petroleum is extracted at low temperature and exported to ashore, along with gas. The plant was modeled and simulated in HYSIM (HYPROTECH-LTD, 1991). The process plants were composed of a separation process as the most inefficient process, comprising an oil heater and three-phase separators, a gas compression process for exporting, which 
has four stages with cooling and liquid separation, an oil pumping process with two pumps, and the crude oil heating that is the most exergy-destruction one.

Svalheim \& King (2003) presented an energy performance study based on the survey of field data from the Norwegian Continental Shelf (NCS). They pointed the compression, exportation and injection (gas or seawater) are the reasons of the large energy demand of processes and discussed the advantages that resulted from applying energy efficiency measures (e.g. operating gas turbines at high load and reducing flaring practices). However, they did not evaluate the effect of utility system, but it was emphasized that the interest of this indicator is limited, because as each oil field has different natural characteristics. The study by (SVALHEIM; KING, 2003) also analyzed other indicators based on environmental impact studies and conventional energy assessments. In order to have a more complete assessment of energy consumption concentrating on utility units, Bothamley et al. (2004) examined the offshore processing options for oil platforms and compared the processing schemes of the platforms in the Gulf of Mexico with offshore in the North Sea regions. The results showed the heating demand was mainly related to the crude oil stabilization, the cooling demand for the oil and gas handling processes, and the power demand for the gas compression.

In addition, Vanner (2005) concentrated on the energy usage over the lifetime of an offshore facility and illustrated that changes in main field, have an impact on the energy intensity of the oil product. The general trend is a higher energy intensity with time, because of the variations in the gas-and water-to-oil ratios, as well as the use of operating scenarios, such as gas lift and water injection, which are employed to enhance the production.

Voldsund et al. (2010) performed an exergy analysis in an oil and gas processing platform in the North Sea. The plant analysis included the power plant and the process plants. The studied separation train is composed of three separators and the separated gas is compressed in three stages with a cooling and liquid separation for each stage which is similar to the most used offshore todays. Their simulations were performed by using Aspen HYSYS and the Peng-Robinson EoS to estimate the properties of mass and energy flows. Figure 2.11 shows a simplified overview of delimiting inputs, outputs and boundaries of a typical offshore in North Sea that was used in (VOLDSUND et al., 2010). The results of this study showed the compression process for injection besides being the highest power consumption, has the highest irreversibility of the plant (66\%), followed by the separation process (20\%) and the re-compression process (11\%) and, finally, the oil pumping process $(3.1 \%)$. 
Figure 2.11 - Simplified overview of an offshore oil and gas platform

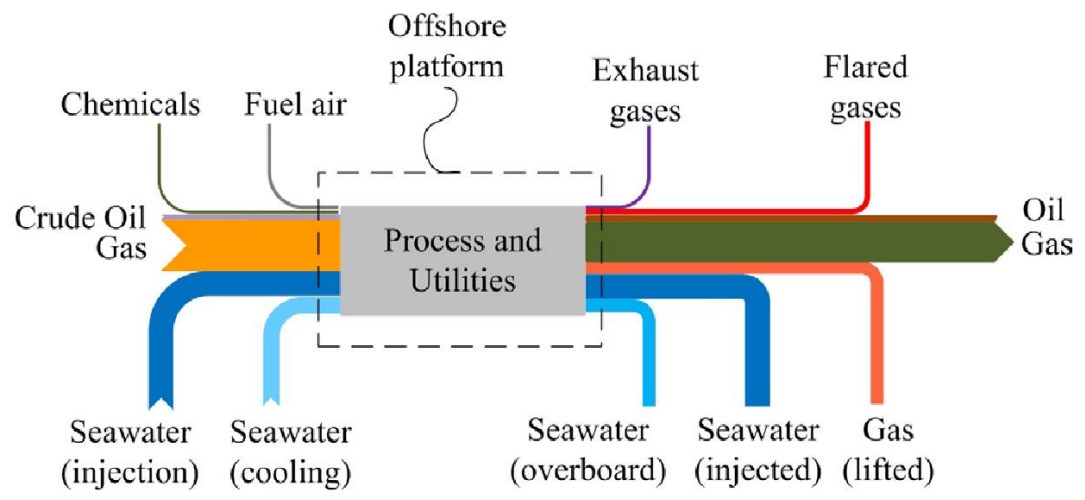

Source: (VOLDSUND et al., 2010)

The International Association of Oil and Gas Producers (IOGP) collected environmental data from its member companies as an annual review in the last 13 years of activity before 2012 . They indicated that in the activities of oil and gas industry, energy planning includes a review of the energy consumption inventory and the variables which affect energy consumption and the selected performance indicators. The report of (IOGP, 2012) proposed several energy indicators accepted by IOGP member companies in order to improve the environmental effects, inducing gaseous emission, energy consumption and flaring as principal issues. In addition, the report showed that the average energy consumption in 2012 was 1.4 GJ (Giga Joules) of energy for each tonne of hydrocarbon produced and Green-House-Gas /GHG emission rates were 160 tonnes of GHG per 1000 tonnes of hydrocarbon production in 2011.

The studies above and others, such as (VOLDSUND et al., 2012),(VOLDSUND et al., 2013b) and (NGUYEN et al., 2013) are the major works in the field of thermodynamics analysis and its application to offshore platforms. It can be noted that the number of studies has increased in the last five years and that environmental law and carbon dioxide emissions are aspects that have promoted a greater amount of research on this topic.

Voldsund et al. (2012), in another work, used three cases to perform thermodynamics analysis. First and second cases with and without anti-surge system, the third case using an increased efficiencies compressor. Additionally, the work included the fuel gas treatment process and the anti-surge control of the compressors. Their result are achieved using Aspen HYSYS and it was shown that the processes with more destroying of exergy are compression and cooling of gas for injection. Voldsund et al. (2013b) detailed the exergy assessment of Voldsund et al. (2010) by adding exergy rate and physical exergy parameters.

Next, Nguyen et al. (2013) described a generic model of an offshore in six cases to simulate the processes and utilities plant, operating conditions and different reservoir fluid 
compositions using Aspen Plus ${ }^{\circledR}$. Their work showed that the highest destroyed exergy occurs in the combustion chamber reaction such as in gas turbine. They compared their results with results obtained by Oliveira-Jr. \& Hombeeck (1997) and concluded that offshore platforms located in different regions may considerably differ due to their process and characteristics.

Nguyen et al. (2014a) and Nguyen et al. (2014b) analyzed the life efficiency of an offshore platform to study the pinch and exergy-based methods to find the destroyed exergy of the system and component. Their results indicated that according to the changes in the flow rate of production and treated fluids, the power and energy demands of systems and subsystems (both main and utilities) also alter for the plateau, mid-decline and end-life cases. Moreover, Voldsund et al. (2014) applied exergy destruction to sub-systems of four different platforms. Their results established that gas treatment and manifold production processes have a major contribution to exergy destruction.

For a Brazilian FPSO, Carranza-Sánchez et al. (2015) performed a thermodynamics analysis of three operation modes in a FPSO. They reported that the highest power consumption of the main compressor was observed for the crude oil composition with high GOR, including condensate components. The authors also concluded that $4.3 \%$ of the gas produced is used for both power and heat generation unit. Next, Nguyen \& Oliveira-Jr (2017) investigated a process synthesis of an oil and gas platforms over different production profiles and feed compositions for a Brazilian pre-salt oil field. In that, the effects of operating parameters assessed on oil and gas production. Their results highlighted that however, oil production and stabilization slightly change the power and heat demands, but they are strongly correlated to gas production.

In a gas condensate processing plant, Mehrpooya et al. (2016) simulated an ethane recovery process in South Pars of Persian Gulf by Aspen HYSYS to find the large sources of irreversibility using exergy analysis. They showed that the operating temperature of compressors can increase irreversibility, and pressure drop as a key parameter can affect the cycle efficiency.

In addition, Gallo et al. (2017) investigated another thermodynamic analysis of a Brazilian FPSO to analyze the performance of a compression system, by considering the operating of components under off-design conditions. They performed the simulation for three conditions of the well fluid composition and mass flow during 25 years (Figure 2.12a). The researchers identified that the major sources of irreversibility and power load variation in each operating year, which can offer opportunities for energy saving. Figures $2.12 \mathrm{~b}$ shows the highest production rate and power demand is between 5th and 10th year of operation. 
Figure 2.12 - (a) Field production along time and (b) Power load variation along time

(a) Field production along time

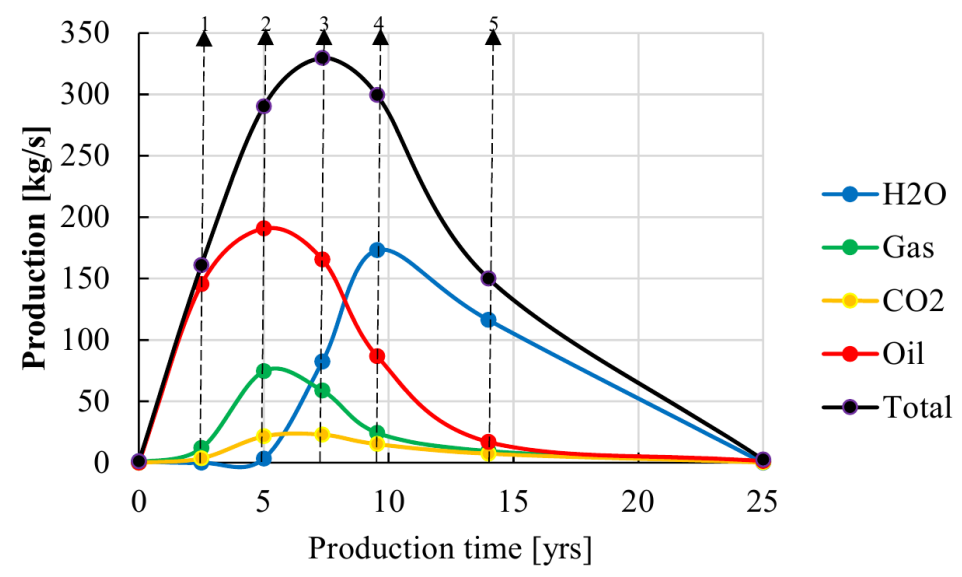

(b) Power load variation along time

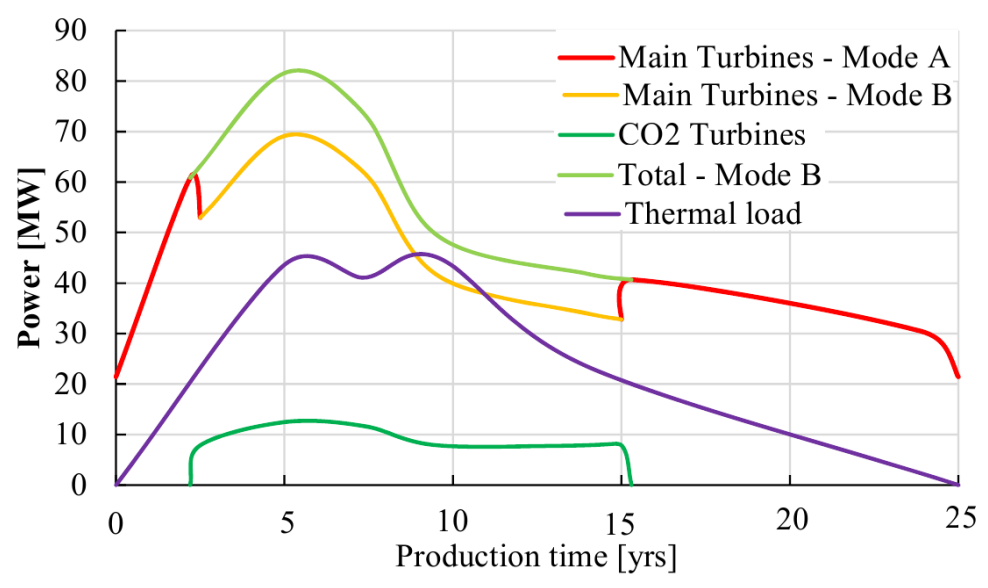

Adapted from (GALLO et al., 2017)

As shown above, the major consumer of energy such as compressors are also the biggest exergy destructive beside of heating processes. Moreover, the heat and generation unit such as gas turbine due to combustion reactions is another exergy destroyer. Note that the gas turbines are responsible for power generation (combustion) and hot water (provided by heat recovery). Thus, it significantly affects energy and exergy consumption alongside production processes such as oil stabilization. The state of the art of indicated researches from these sections are showed in Tables 2.1, 2.2 and 2.3. 


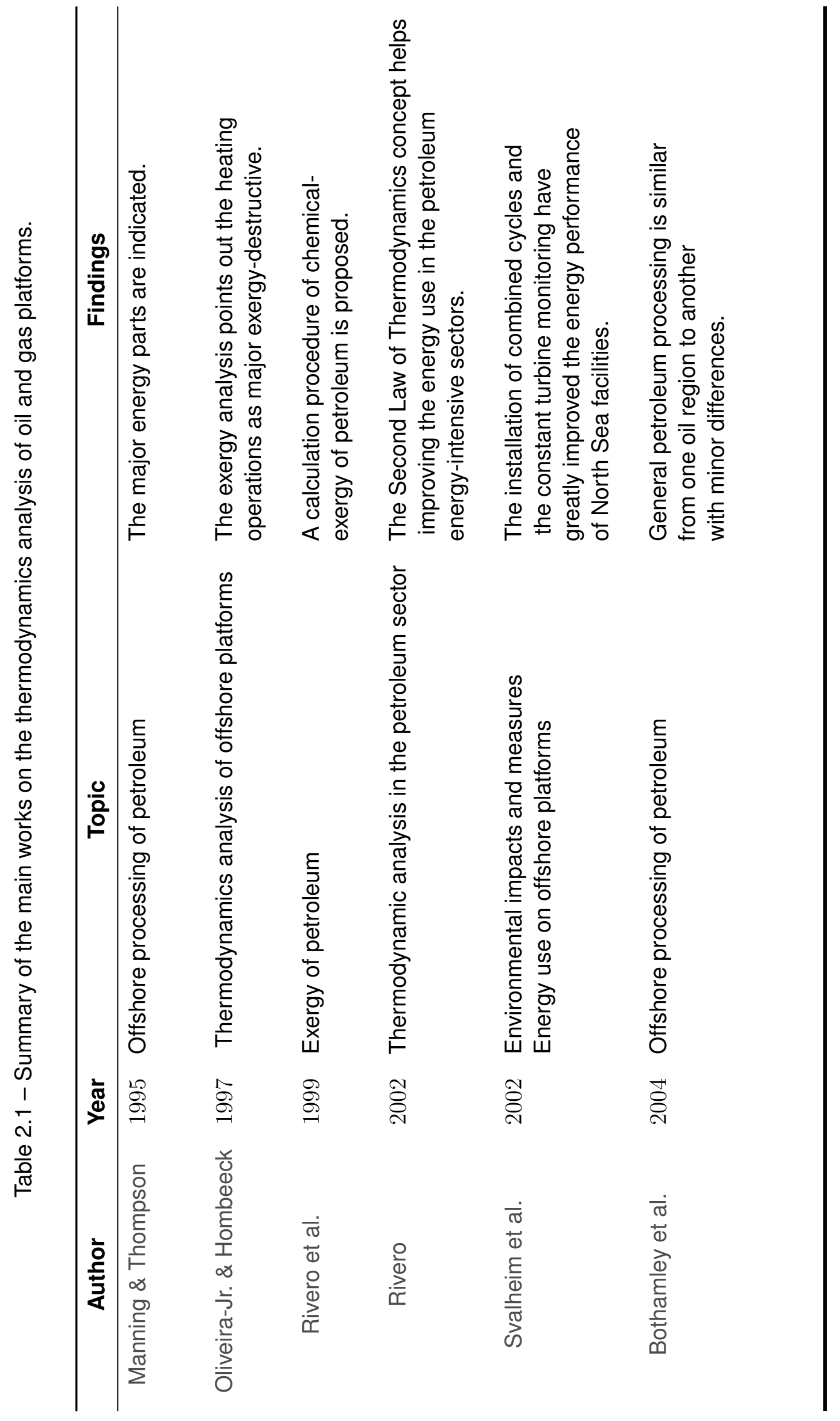




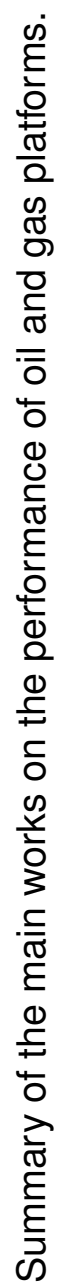

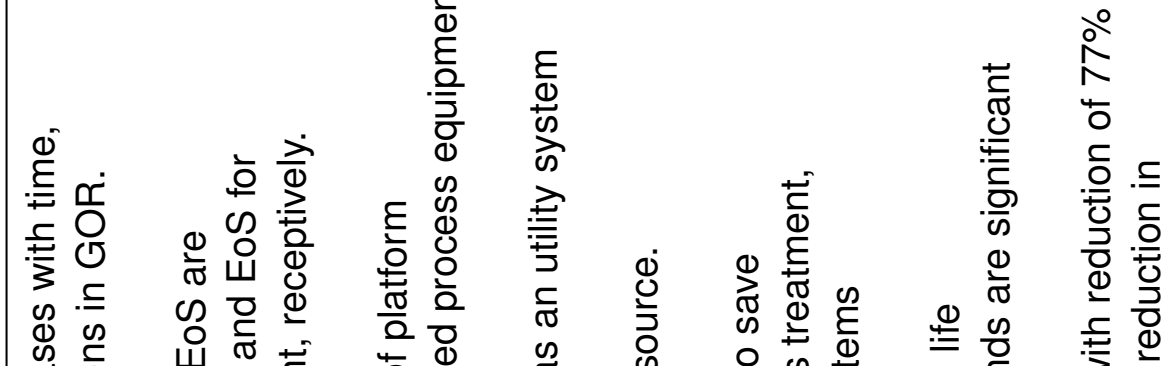

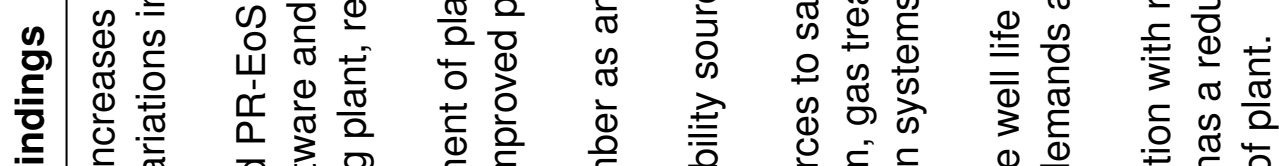

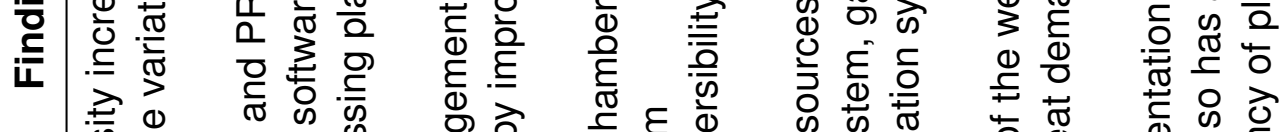

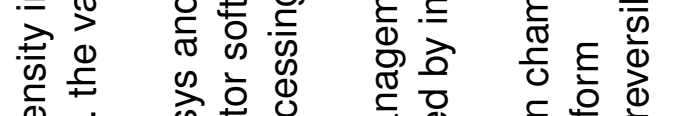

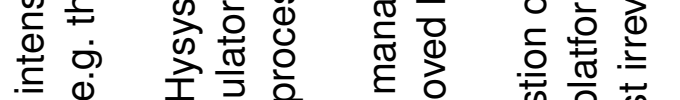

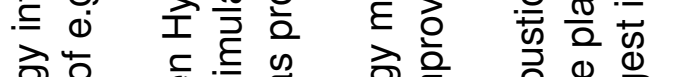

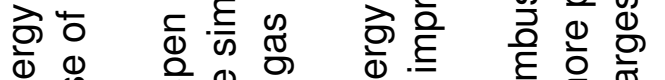
ब ब ঠ్ర

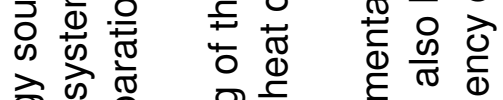

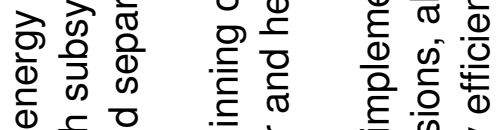
๑ั

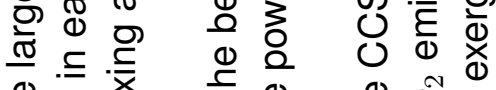

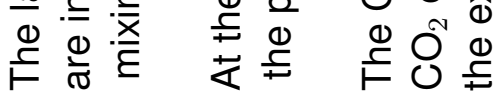

$\stackrel{\infty}{\frac{\infty}{0}}$

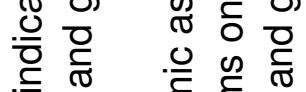

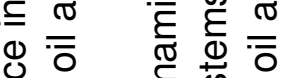

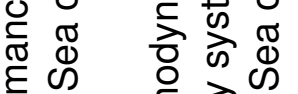
하 ठำ

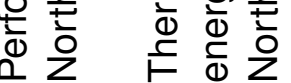

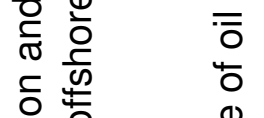
垔 क 


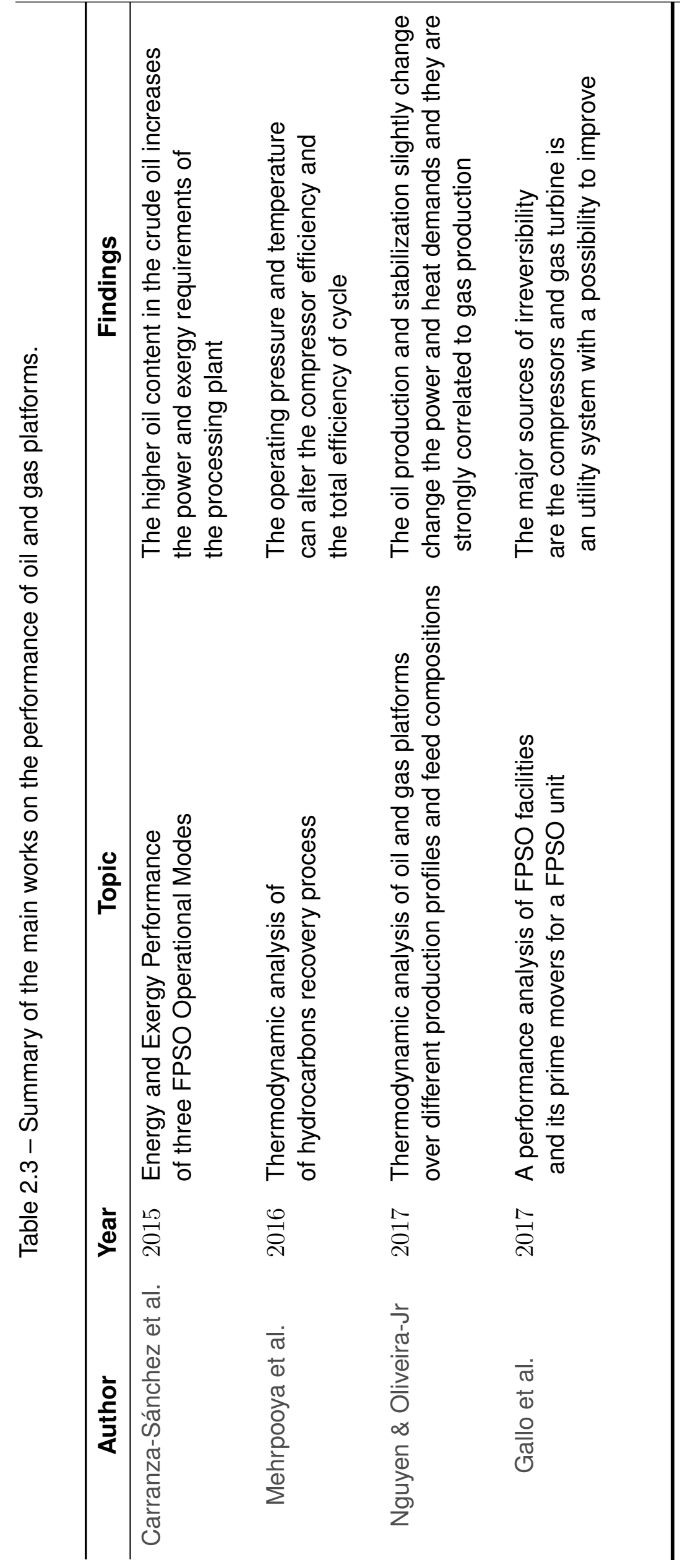




\subsection{Process Optimization in Processing Plants}

Process optimization means adjusting or modulating a process so as to optimize some specific set of variables without violating some constraints. The minimization of cost and energy consumption, and maximization of throughput and/or efficiency are the main purposes. By the same token, process optimization is the final and most important task, and is essential for improving the performance of the process. Hence, it has attracted the attentions of engineers and researchers.

In this section of the literature review, a large number of researches and techniques of process synthesis, modeling and optimization of the various industrial processes, including liquefied natural gas and oil and gas processing plants are investigated. However, there are very few optimization studies concentrating on a thermal system of an offshore, particularly on FPSO operation range, scale and its limitations.

Accordingly, in general, Process Optimization is divided into two main parts: 1- Process synthesis and modeling, and 2- Optimization challenges related to optimal scheduling of the different unit tasks to perform the overall process objective (or objectives).

\subsubsection{Process Synthesis and Modeling}

Process Synthesis is a research area which interest of it has grown over the last decades for chemical processes. However, very few works deal with the systematic synthesis of an entire oil and gas platform. In this subsection, a review of the existing literature of process synthesis and its importance for modeling chemical processes is detailed.

A few decades ago, Rudd et al. (1973) explained that processing systems are characterized by two distinct features. The first one is chemical and physical properties of process components and interconnections between components, and the second one is the capacities and operating conditions of these process components. Nonetheless, to synthesize of a process due to finding an optimal configuration, a directed search over the feasible alternative configurations as well as over the design variables is necessary.

In the importance of process synthesis in system design, Bradley et al. (1977) also stated that the engineer must formulate the problem and the constraints imposed, and then highlight the results of the model in the light of gained experiences and intuitions, recognizing the model restrictions. After reviewing of 190 papers of process synthesis, Nishida et al. (1981) explained that Process Synthesis is the first step in process plant design by the systematic generation of alternative process flow sheets and the selection of one or few configurations and parameters to optimize a given objective(s) function.

In the design of chemical processes by process synthesis, Colmenares \& Seider (1989) showed that the performance of the utility system directly influences the operating cost and 
efficiency of a process.

Additionally, Smith (2005) mentioned that the overall purpose of process synthesis is to design the processes with the suitable physical and/or chemical transformations (in main or/and utility systems), which are necessary to achieve the desired outputs and productions. It can either be performed by designing new facilities or modifying existing ones (retrofit).

After all, recently, the process synthesis has been the inseparable part of optimization procedures in chemical processes of the most researches and works. Then, the mathematical modeling is used to set the complexes structure and chemical process to calculate the objective functions based on real variables and constraint in a nonlinear programming formats (ADJIMAN et al., 1998). In the following, the implemented process synthesis methods in various studied cases of industrial cycle focusing on oil and gas processing plants are raised.

Next, Lee et al. (2002) proposed a novel method to select the refrigerant compositions based on the combination of nonlinear programming (NLP) and thermodynamic analysis. They developed a systematic synthesis method as a tool for completing the design of a mixed-refrigerant cycle. Their case studies demonstrated up to $25 \%$ saving in the shaft works power demand compared to the commercial process. Although, the complex procedures were time-consuming.

To develop a nonlinear model to connect the superstructure and process components, the process synthesis should be detailed in a methodology framework. Wang et al. (2012) presented a new methodology for process synthesis of LNG liquefaction to minimize energy consumption. A procedure using MINLP (mixed-integer non-linear programming) model is developed for a C3MR LNG plant and it is solved in GAMS by a solver called LINDOGlobal. The synthesis model comprise of mass and energy balances employed in the optimization model. They claimed that their methodology reduced energy consumption by approximately $13 \%$ and as shown in Figure 2.13, the validation thermodynamics properties and numerical solution results were examined by Aspen Plus ${ }^{\circledR}$. 
Figure 2.13 - The presented methodology framework in Wang et al. (2012)

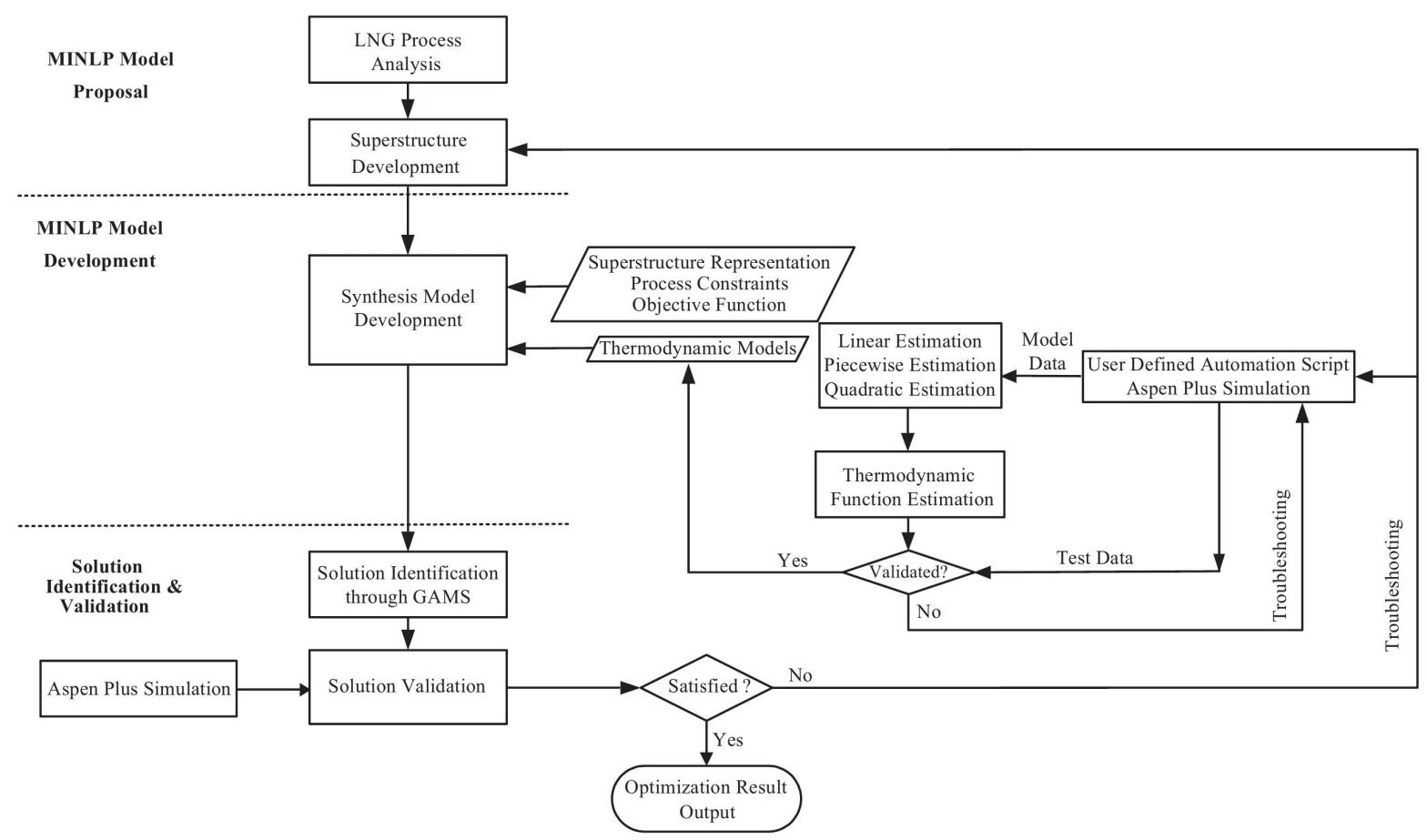

Source: (WANG et al., 2012)

Willersrud et al. (2013) studied the application of methods to maximize the total oil production of an offshore in the short time scale and by a process synthetic.In this work, it was employed two methods of nonlinear model predictive control (NMPC) in the optimization model. First, they used unreachable set-points method to maximize oil production with a constant GOR in the wells and then, exact penalty function and infeasible soft-constraints. The infeasible soft-constraints method provided fewer tuning parameters in an easier principle set up. They showed that the total oil export could increment by around $70 \mathrm{Sm}^{3} /$ day, corresponding to a yearly increased revenue of $16 \mathrm{M} \$$. However, their results proved how pressure control as a decision variable could increase oil production without optimization.

Furthermore, Wang et al. (2014a) and Wang et al. (2015) performed synthesis and design optimization of thermal power for a LNG mixed refrigerant processes. They modeled a MINLP based on the relevant superstructures systems and global optimal system configuration, to improve performance and energy conversion system.

The study by Silva et al. (2015) is another attempt to maximize the production with multiple routing decisions, pressure constraints and lift-gas distribution based on an FPSO platform.They developed a nonlinear model with automatic well-manifold routing; the problem was formulated as a Mixed-Integer Linear Programming (MILP) using piecewise-linear models to approximate the nonlinear functions for production optimization. 
Nguyen et al. (2016f) focused on the development of integrated and intensified petroleum processing plants, including the process steps, transformations and interconnections of relevance as a synthesis of preliminary system designs for offshore oil and gas production (Figure 2.14). They formulated three types of petroleum processing plants as a starting point and coupled their developed model in Aspen Plus with Matlab to perform multi-objective optimization routines and uncertainty assessments by considering the technical, energy and economic criterias. In all the cases, the results showed that the system performance were strongly depended on the level of mass integration within the platform; the recovery of the light and heavy hydrocarbons were impacted by the number of separation stages and the additional heat exchangers. In this work, the effects of separated liquid streams from each separation train on other stages and recovered volatile oil to separation train are not mentioned. In addition, the influence of gas compression steps on pumping is not clarified. Finally, understating the magnitude of each variable on the objectives can be a very important step in process synthesis of any chemical processing plant.

Figure 2.14 - Generic superstructure of an oil and gas processing plant. $\mathrm{S}_{1-2}, \mathrm{~S}_{3-6}$ and $\mathrm{S}_{7-14}$ indicate separation configurations in one, two and three stages, while the numbers indicated for the other steps, e.g. $R_{1}, C_{1}, T_{1}$ and $P_{1}$, are the number of re-compression, compression, treatment and pumping stages, respectively.

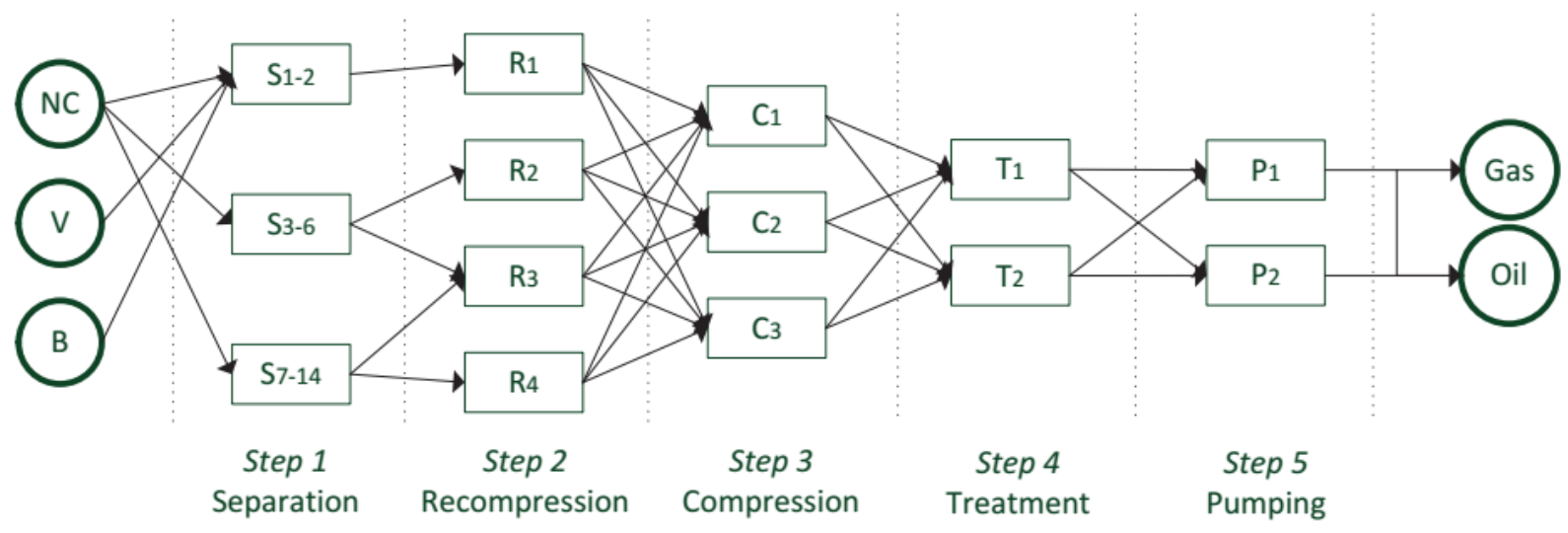

Source: (NGUYEN et al., 2016f)

Finally, Diban \& Foo (2017) presented a process integration method of an applied heating utility system for an offshore oil platform. A revised Nearest Neighbor Algorithm (NNA) was used for designing the heating utility system. The authors indicated that in the case of no available data of heat exchangers, this approach is useful for a preliminary design. 


\subsubsection{Sensitivity analysis and Optimization of Industrial Processing Plant}

To join the nature of the industrial process system and oil and gas process, this subsection is a review of existing papers about sensitivity analysis and optimization in an industrial process. The origin of any industrial optimization study stands in a presumption that the feasible improvements can be produced in a controllable system. The first point for solving a problem, is to assess all the facts about a problem considering their interactions and restrictions. Aute (2014) defined that the optimization of thermal systems is usually performed with a mixture of technology decisions and the optimization of specific properties of selected components.

Screening or sensitivity analysis is often a preliminary step in any optimization procedure that uses a large number of input parameters as decision variables. The main objective of the screening analysis is to identify the most important contributors to increase/decrease an output value. Hence, to have an intelligent optimization process, using sensitivity analysis is unavoidable. In this subsection, sensitivity analysis and some of the optimization methods in industrial processing plant are indicated.

Sensitivity analysis is a generalized methodology to identify and localize influential variables for a conceptual process design or optimization process under uncertainty. Similarly, it is divided to a methodology into three steps: process design and sensitivity analysis to identify the effective variables, elimination of non-influential input variables, determination and regionalization of critical variables or/and performing optimization procedure with these given steps (LUCAY et al., 2015).

Global sensitivity analysis has been applied to different chemical processes in order to provide a quantitative ranking of critical parameters (VERMA et al., 2017). There are also many studies which applied sensitivity analysis as a tool to evaluate the process structures and operational behaviors of chemical and industrial cycles (HATCHER et al., 2012; JIANG et al., 2012; CHU; HAHN, 2013; SEPÚLVEDA et al., 2014). Additionally, Sensitivity analysis provides the important parameters that should be considered in input variables of an optimization process and a responses surface of output values ( indirect optimization). For example, Gao et al. (2010) proposed nitrogen expansion liquefaction process with propane pre-cooling. The authors performed the sensitivity analysis of several parameters on the liquefaction process to find the space solution, without performing a systematic optimization of the process.

Optimization of LNG plants and their related subsystems is one of the objectives that there are several works related to it are published. AS the LNG is an energy-intensive process, the most of works used screening analysis for operating parameters to minimize the power and energy consumption of an LNG plant.

For a mixed-refrigerant LNG process, Hatcher et al. (2012) proposed a systematic analysis of optimization formulation of the cycle. They used refrigerant flow rate, outlet pressures 
of expansion and compression, and outlet temperatures of heat exchangers for natural gas stream as input variables. Moreover, they implemented sensitivity analysis to show which parameter is most effective on energy minimization. Chu \& Hahn (2013) also used existing global sensitivity analysis techniques to illustrate the influence of selected variables on the optimal experimental design of process systems. In addition, Jiang et al. (2012) studied the sensitivity of the methane gas production rate during depressurization from hydrate reservoirs by the operation parameters using a numerical model of of reservoir fluid, thermodynamic and chemical relations. However, varying of output by changing the input parameters is simply considered as a sensitivity method that, it could not be a robust strategy.

In another optimization research of LNG processes, Hwang et al. (2013) used the hybrid optimization method (GA+SQP) to find the optimal operating condition of a DMR cycle at LNG FPSO. The implementation of SQP method increased the convergence of GA. The required power at the obtained minimum condition decreased by $34.5 \%$ compared with the patent, and by $1.2 \%$ compared with the conditions obtained from the relevant baseline.

Moreover, in an optimization of capital cost and energy consumption, Wang et al. (2014b) used a sensitivity analysis in C3MR and DMR processes. Their objectives were to identify the effect of the variation of the operating and cost parameters on reducing energy consumption and total capital expenditure (CAPEX) including operating expenditure (OPEX) using PR-EOS. Then, they performed an optimization procedure using box methodology and controlled elitist GA to improve the exploration of the design space.

There are two researches of using sensitivity analysis in the different applications of industrial processing. Xia et al. (2014) examined the effect of some key parameters on the system performance of a solar-powered transcritical $\mathrm{CO}_{2}$ cycle with $\mathrm{LNG}$ as a heat sink based on the recovery of cryogenic energy of LNG. Parametric sensitive analysis is carried out to identify the contributors of key parameters on the performance. Then, the parametric optimization is carried out to find the optimal performance using GA. Sepúlveda et al. (2014) also analyzed the impact of statistical distributions of input variables data on the resultant distribution by global sensitivity analysis in mineral processing circuits.

In a $\mathrm{N}_{2}-\mathrm{CO}_{2}$ expander LNG process, Khan et al. (2014a) minimized the total compression energy requirement for $N G$ liquefaction. The operating pressures (suction and discharge) of $\mathrm{N}_{2}$ cycle, $\mathrm{CO}_{2}$ degree of super-heating and $\mathrm{N}_{2}$ flow rate were selected as variables to analyze compression shaft work of $\mathrm{N}_{2}$ compressor, $\mathrm{CO}_{2}$ compressor and $\mathrm{N}_{2}$ expander. $\mathrm{A}$ mono-objective optimization algorithm made by in-depth process analysis integrated with a simulation to fulfill $\mathrm{N}_{2}-\mathrm{CO}_{2}$ expander process. The optimization results revealed energy savings of $15 \%$ compared to the base case and $7 \%$ increase in the refrigerator exergy efficiency. However, the operating and capital cost is not considered in the optimization algorithm. 
Moreover, Khan et al. (2014b) developed a novel liquefaction of NG cycle. Then, the separation of $\mathrm{NG}$ liquids was performed using energy efficient thermally coupled distillation schemes. They optimized the energy consumption of compressors by altering the refrigerant composition and operating pressures using knowledge-based optimization (KBO) methodology. Their results demonstrated an improvement of $9 \%$ in plant energy requirement of as compared to the baseline. However, it would be more interesting, if the implemented method was compared with some convenient method such as GA to explain the advantage and disadvantage of KBO method.

Figure 2.15 shows, a systematic optimization procedure using commercial simulator (Aspen HYSYS) and GA (by Matlab) that was presented by (HE; JU, 2014).

Figure 2.15 - The framework of the process optimization with GA.

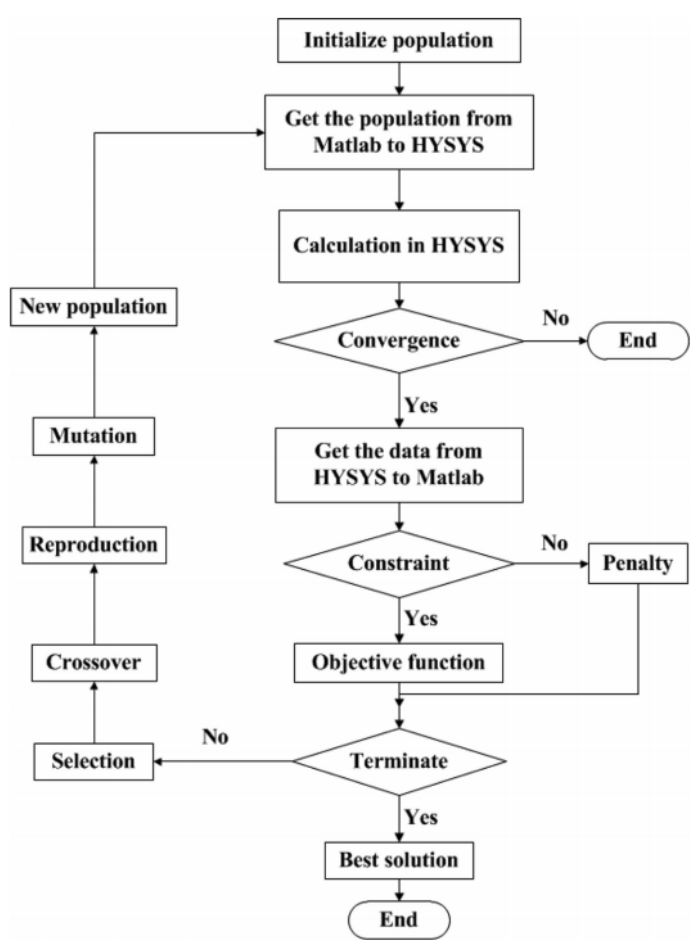

Source: (HE; JU, 2014)

They modelled a novel mixed refrigerant cycle with NGL recovery unit. In their paper, the calculated energy consumption was considered as the objective function and optimization process performed just after the convergence of numerical simulation (Figure 2.15). In addition, He \& Ju (2015) proposed four configuration strategies of expansion liquefaction cycle for distributed-scale LNG plant to evaluate the liquefaction cycles and exergy analysis for distributed scale LNG plant. They configured sixteen feasible liquefaction cycles to maximize FOM (figure of merit) as an objective function for optimal synthesis. To solve the optimization problem, GA is selected. They coupled again Aspen HYSYS as a simulator with MATLAB as an optimizer. Their results showed a case with two cycles, namely R410A. The 
pre-cooling cycle and the parallel nitrogen expansion cycle formed the optimized liquefaction cycle.

The effective variables on objective function, such as the pressures and the molar flow rate of the mixed refrigerant, the inlet temperature of the demethanizer, the inlet temperature of the demethanizer, and the inlet pressure of the deethanizer were considered. Their results were established by a $9.64 \%$ reduction in energy consumption as compared with the baseline, and $11.68 \%$ in molar flow rate of mixed-type refrigerants. Likewise, Soffiato et al. (2015) used Matlab to run optimization method and to couple with simulator. In that, the problem formulation was performed by EES and SQP was carried out as the gradient-based optimization method in a two-stage ORC. They defined the system efficiency as objective function subject to technical constraints. Moreover, Mosaffa et al. (2017) investigated four configurations: three single-stage ORC (simple, recuperative and regenerative) with $\mathrm{n}$-pentane as the working fluid and one two-stage ORC with n-pentane/n-butane as the fluid combination. However, the fluid optimization was performed with a parametric optimization of six effective parameters. They used the DIRECT algorithm in the EES software for optimization.

Feng et al. (2015) performed a thermo-economic analysis for RORC and BORC using multi-objective optimization by using the Non-dominated Sorting Genetic Algorithm (NSGA-II). Next, they applied the Pareto Frontier solution with bi-objective to compare the corresponding solutions of a single-objective. The thermodynamic performance, exergy efficiency, and levelized energy cost (LEC) for BORS and PORC are assumed objective. The constraints were defined based on pinch-point temperature difference and energy balance. In this study, the key parameters, such as evaporator outlet temperature, condenser temperature, degree of superheat, pinch point temperature difference, and degree of supercooling are selected as the decision variables. In accordance with the results presented; RORC had $8.1 \%$ higher exergy efficiency and LEC was about $21.1 \%$ more than in other cycles.

To find the optimum refrigerant composition and operating pressures for compression energy requirement, and application to LNG plant, Park et al. (2015a) used the modified Coordinate Descent Methodology (MCD) (a derivative-free optimization algorithm). They expressed that in Korean Single Mixed Refrigerant (KSMR) process, energy requirement of the compression is strongly a function of refrigerant composition and its operating pressures. Thus, optimization of these parameters can reduce the power consumption significantly. Their results of optimization showed $40 \%$ and $11 \%$ in energy savings, compared with the representative base cases. In addition, they compared the suitability, calculation time and ease of implementation of the MCD algorithm with PSO (Particle Swarm Optimization) and NSGA-II that are the advantage of their methodology. Furthermore, in a process synthesis to make a new design Park et al. (2015) evaluated a novel natural gas liquid (NGL) recovery process with lower equipment numbers that are suitable for floating applications such as 
offshore by comparing several representative patented NGL recovery processes. They carried out the techno-economic analysis to evaluate a variety of processes for comparison with the proposed novel one. Their steady-state simulations were used to screen the alternatives and to develop a process with better heat integration and better separation efficiency.

A configuration design to satisfy the profitability and reliability of chemical processes was the subject of few researches in the last decade. Getu et al. (2015) made an optimal decision to investigate the risk of natural gas liquid (NGL) recovery processes. They analyzed the performance with respect to the uncertainty from the plant inlet of six representative NGL recovery processes. They simulated cases by ASPEN HYSYS and formulated the optimization models for each process scheme using General Algebraic Modeling System (GAMS). They considered the important topic of uncertainty and its incorporation in the optimization of natural gas liquid (NGL) recovery plant designs. Their optimal decision was made based on the profitability and reliability of holding the process constraints. Mehrpooya et al. (2016) also performed a sensitivity analysis in a gas processing plant and showed that pressure drop is a significant permanent parameter with a great effect on energy consumption and performance of the process. I addition, the exergy analysis was used to increase the reliability of system.

Next, Fergani et al. (2016) performed an evolutionary multi-objective optimization by the MOPSO (Multi Objective Particle Swarm Optimizer) algorithm that uses multi-criteria exergy to optimize an Organic Rankine Cycle in cement industries. The Pareto front of a multi-objective optimization is used for the range of optimal point and Particle swarm optimization method. They considered three working fluids for ORC, the turbine inlet pressure, the pinch point temperature difference in the evaporator, the pinch point temperature difference in the condenser, and the inlet temperature of the heat transfer fluid as decision variables. Cyclohexane had the best exergoeconomic results and benzene was the best from the exergo- environmental point of view for cycle turbine.

With the increase of capability in computing power of computers, simulators and optimizers, the number of selected operating parameters as input variables in studies have also increased. Moreover, the systems with more complexity have been investigated ((HE; JU, 2014), (KHAN et al., 2014a), (KHAN et al., 2014b), (HE; JU, 2015), (FENG et al., 2015), (PHAM et al., 2016), (YAO et al., 2016) and (KWAK et al., 2018)). Among them, some of works have conducted the novelty in applied simulation or optimization which are detailed and discussed in down.

Pham et al. (2016) examined the effects of the flow rates of the each refrigerant components and the working pressures on the liquefaction efficiency. It was also investigated the interpreting the geometric pattern of the temperature difference (in a plot) between the hot and cold composite curves for a heat exchanger of cryogenic cycles. The minimization of 
energy compression consumption on modified single mixed refrigerant cycle of natural gas liquefaction targeted for offshore applications is elected as objective function. They carried out a process to choose the impossible "perfect" beginning point and a search sequence of variables for the coordinate descent algorithm using a multivariate Coggin's algorithm. The results presented a $21.9 \%$ and $18 \%$ reduction in compression energy compared to two selected base cases.However, the authors in abstract indicated that "knowledge-inspired hybrid optimization approach with a robust convergence" has been use, but There is no any information or graph about them in the paper.

Yao et al. (2016) also applied NSGA-Il to obtain maximum thermodynamic efficiency by a novel integration cooling, heating, and power system from a small-scale compressed air energy. They also studied the effect of key parameters on system performance by sensitivity analysis. They concluded that the incremental of inlet temperature, the pressure of the turbine and effectiveness of the heat exchangers enhance the thermodynamic performance and $52.51 \%$ of exergy efficiency can be achieved.

An optimization process for a boil-off gas (BOG) re-liquefaction cycle applied by (KWAK et al., 2018) in LNG fuelled ship. They used Unisim to simulate and GA to find optimal operating conditions of proposed process. BOG compressors of baselines were reported as one of the key design issues, and optimization procedure found $11.4 \%$ and $20.8 \%$ reduction in total power consumption of proposed compressors. Sensitivity analysis is also fulfilled to understand the contributions of BOG operating pressures, composition, and re-liquefaction rate on BOG re-liquefaction processes. However,the implemented sensitivity analysis is performed after optimization procedure and it is a screening of the effect of input parameters on optimal results.

As it was observed, the sensitivity analysis can show more effective parameters in order to remove not influential parameters. Moreover, due to complexity of industrial processes and supporting all type of variables in GA, it is a more commonly used method in optimization in these processes. In conclusion, there are many works that because of using the same process, method and illustrated system are not indicated here.

\subsubsection{Sensitivity analysis and Optimization of Oil and Gas Processing Plants}

The growing demand of gas-petroleum and the maturing of existing oil fields have compelled its operators to invest in new researches and technologies to optimize their production processes. Thus, the oil and gas industries have spent a lot of time and effort in optimizing of processing plant in order to improve the efficiency of applied thermal systems and desired productions. Similarly, the optimization techniques have been applied to almost all aspects of the oil and gas industry. The selection of appropriate objective functions, key decision variables, optimization method and constraints are the principal structure of any optimization 
work.

In this subsection, the applications of optimization techniques in various objectives, such as power and energy consumption with environmental effect and production system design and operations are reviewed. Consequently, the decision to perform appropriate steps for the present thesis is made in 2.3 overview section.

Production optimization has been a topic of interest in academic and industry for many years. The main endeavor was to model the mathematical tools which can help decision-makers to select the best production plan. the maximization of liquid hydrocarbons production by stabilization of volatile components is under attention recently.

For those reasons, an accurate methodology for optimizing the separator pressure in a crude oil production unit was suggested by (BAHADORI et al., 2008). They explained that due to different temperatures in winter and summer seasons, the dissolved gases at high pressure tend to come out from liquid phase at low pressures. To address this issue, they used flash calculations to determine optimum pressures of separators at different stages of separation and consequently to optimize the operating conditions without installing any additional equipment. They performed a hierarchical optimization method to minimize GOR sequentially with the stage order of the separators using Aspen HYSYS. However, they did not explain clearly the procedure of finding the optimum pressures. Willersrud et al. (2013) also reported that pressure control can be used to increase oil production without any optimization procedure using the active constraint of the pressure at inlet separator. The authors reported an increase in oil production of $143 \mathrm{Sm}^{3} /$ day compared to no control.

Next, Ghaedi et al. (2014) implemented an optimization procedure for operating pressures of separators in multistage production units. They used Genetic Algorithm to optimize the separator pressure for a crude oil production unit with four separation stages and gas condensate production unit with three separation stages. Compared to a baseline condition, their results showed the improvement of $2.4 \%$ and $8.6 \%$ for crude oil and gas condensate productions, respectively.

In a Korean case of offshore plant, Kim et al. (2014) used Aspen HYSYS and CMA-ES (Covariance Matrix Adaptation Evolution Strategy) as a stochastic optimization in the production for an oil offshore platform. The Peng-Robinson EoS model was used in this work as a suitable one for predicting the phase equilibrium and thermodynamic properties for a High GOR ceude oil. They determined the design variables for the crude oil separation process simultaneously, to achieve the maximized profits. They concluded condensate recycling increases the profits more than increasing the number of separation stages. Kylling (2009), also optimized the profits in terms of oil sales and compression cost based on equation-based modeling and brute force optimization with a simpler method.

In the modeling of an oil and gas processing offshore, one Norwegian filed with volatile oil, 
and two Brazilian fields, Nguyen \& Oliveira-Jr (2016g) studied three petroleum feeds by different content of gas and carbon dioxide contents. Then, they carried out an optimization process using GA by coupling Matlab and Aspen Plus. The operating temperatures and pressures beside the number of separation stages are considered as variables. Their results indicated the integration of a three stage separation train and the heaters are very beneficial for separation performance of hydrocarbons. However, the authors did not explain the function and sequences of the separation stages.

Allahyarzadeh-Bidgoli et al. (2017b) optimized the fuel consumption of a Brazilian FPSO for petroleum composition with maximum oil and gas content by using NSGA-II. The optimal operational parameters found by the optimization procedure presented a reduction of $4.6 \%$ in fuel consumption and indirectly increased the hydrocarbon liquids by $1.95 \%$ as compared to the baseline operational condition of a Brazilian FPSO plant.

As it was shown in the literature review of optimization in desire production, the new power demand and energy in optimization model are not investigated. While, optimized operating pressure and temperature in order to increase production, regarding operating condition of compressors, pumps, and heat exchangers could lead to more energy consumption.

In optimization of an equipment and the effect of improvement on all of cycle, Pierobon et al. (2013) investigated a multi-objective optimization to improve design and working fluid in an organic Rankine cycle offshore. Thermal efficiency, compactness, and net present value are studied by employing Genetic Algorithm. They calculated the waste heat recovery of gas turbine. Their results showed almost $4 \%$ increase in thermal efficiency by using acetone as the working fluid and the net present reached from 17.7 to $19.8 \mathrm{M} \$$ and for Cyclopentane 27-28.1\% thermal efficiency and 19.7-20.1 M\$ for net present value. Next, Pierobon \& Haglind (2014a) presented two new design to recover exhaust heat from offshore platforms. Then, it were considered maximizing the economic revenue, the power production, and the compactness of air-bottoming cycle for waste as multi-objective. They used the Genetic Algorithm and the theory of power maximization to obtain the optimal point. More than 15 variables such as turbine inlet pressure, Pinch point recuperator, the Inner diameter of the tubes in HEs, etc were applied in optimization process. They found about $16 \%$ more boosting for the power of gas turbine (SGT-500) and an increase of $5.2 \%$ in thermal efficiency. In another research, Pierobon et al. (2014b) performed a study to assess three waste heat recovery (WHR) units for a gas and oil offshore platform. They compared Air bottoming cycle power, Rankine cycle and organic Rankine cycle as WHR. The objective functions are net present value, weight and annual carbon dioxide emissions. They assumed the combined cycle to have a turbine gas and a bottoming cycle unit. The multi-objective optimization used a controlled elitist GA for 16 variables to analyze such as inlet pressure of gas turbine, Pinch point recuperator, Inner diameter of the tubes in HEs, etc to select simultaneous optimization solutions. This work showed that combined cycle design-point and part-load present better 
thermal efficiency.

Several authors have performed optimization of the thermal system of offshore oil and gas processing plants focusing on efficiency enhancement of offshore plants by using new technologies that made imposed costs. Nguyen et al. (2016a) performed a study to assess several technologies for improving energy efficiency and reductions in $\mathrm{CO}_{2}$-emissions as a second indicator in a Norwegian offshore. They applied some suggestions in re-designing the processing plant and performed an optimization problem for each. They included:

(i) the installation of the production manifolds with multiple pressure levels, (ii) a very simple energy and process integration, (iii) the multiphase expanders, (iv) the gas recirculation around the compressors, ( $v$ ) the exploitation of low-temperature heat from the gas cooling steps, (vi) the downsizing or replacement of the existing gas turbines, and (vii) the use of the waste heat from the power plant. A multi-objective optimization was performed applying the GA developed for complex integrated energy systems. They showed the integration scale can decrease the external heating demand, but this would result in an additional operating issue. In addition, installing a waste heat recovery in a small gas turbine leads to a more efficient power generation system and, consequently, to lower $\mathrm{CO}_{2}$ emission. Their results revealed that reaching up to $15-20 \%$ energy saving and reduction in $\mathrm{CO}_{2}$-emissions. However, the thermoeconomic and cost estimates for alternative configuration and technology were not considered for each plant in this work.

Next, Nguyen et al. (2016e) assessed another solutions based on the energy-efficient and environmentally friendly to reduce $\mathrm{CO}_{2}$-emissions in the oil and gas offshore sector. They suggested some new installation to apply such as (i) waste heat recovery, (ii) the $\mathrm{CO}_{2}$-capture unit and (iii) the platform electrification in a North Sea platform as a case study for analyzing and comparing the minimization of the external energy demands and associated operating costs. The model was formulated as a MILP (Mixed Integer Linear Programming) problem. Their indicators were thermodynamic, economic and environmental as a multi-objective optimization routine based on Genetic Algorithm. The modeling and analysis were performed by Aspen Plus which represented the physical and chemical processes in oil and gas processes, as well as in $\mathrm{CO}_{2}$-capture systems. The optimal system configurations were illustrated under the form of a Pareto optimal frontier, which separates the research domain into the feasible, but sub-optimal solutions, the feasible and Pareto-optimum solutions, and the infeasible ones. They revealed the integration of the steam network in waste heat recovery can be profitable, with an increase of the power generation capacity of up to $8 \mathrm{MW}$ and a greater gas export of up to $16 \%$.

In addition, Liu et al. (2016) also investigated a theoretical analysis for the thermal performance of the waste heat recovery system for FPSO facilities. They used the ideal air standard Brayton cycle for a diesel engine to analyze thermal performance. The energy efficiency and thermo-economic index were performed with the diesel system fan and without the fan. 
They showed the fan can increase efficiency and energy saving; however, with regards to the limited space in FPSO, it can be removed.

Finally, Reis \& Gallo (2018) explored some alternatives for waste heat recovery in an FPSO platform using an organic Rankine cycle (ORC) to meet a required heat demand for two different cycle configurations. The authors used the Genetic Algorithm for electric power demand minimization. They reported a significant average reduction of $22.5 \%$ in fuel consumption during the lifetime of the FPSO.

The state of the art of indicated researches from these sections are showed in Tables 2.4, 2.5 and 2.6. 


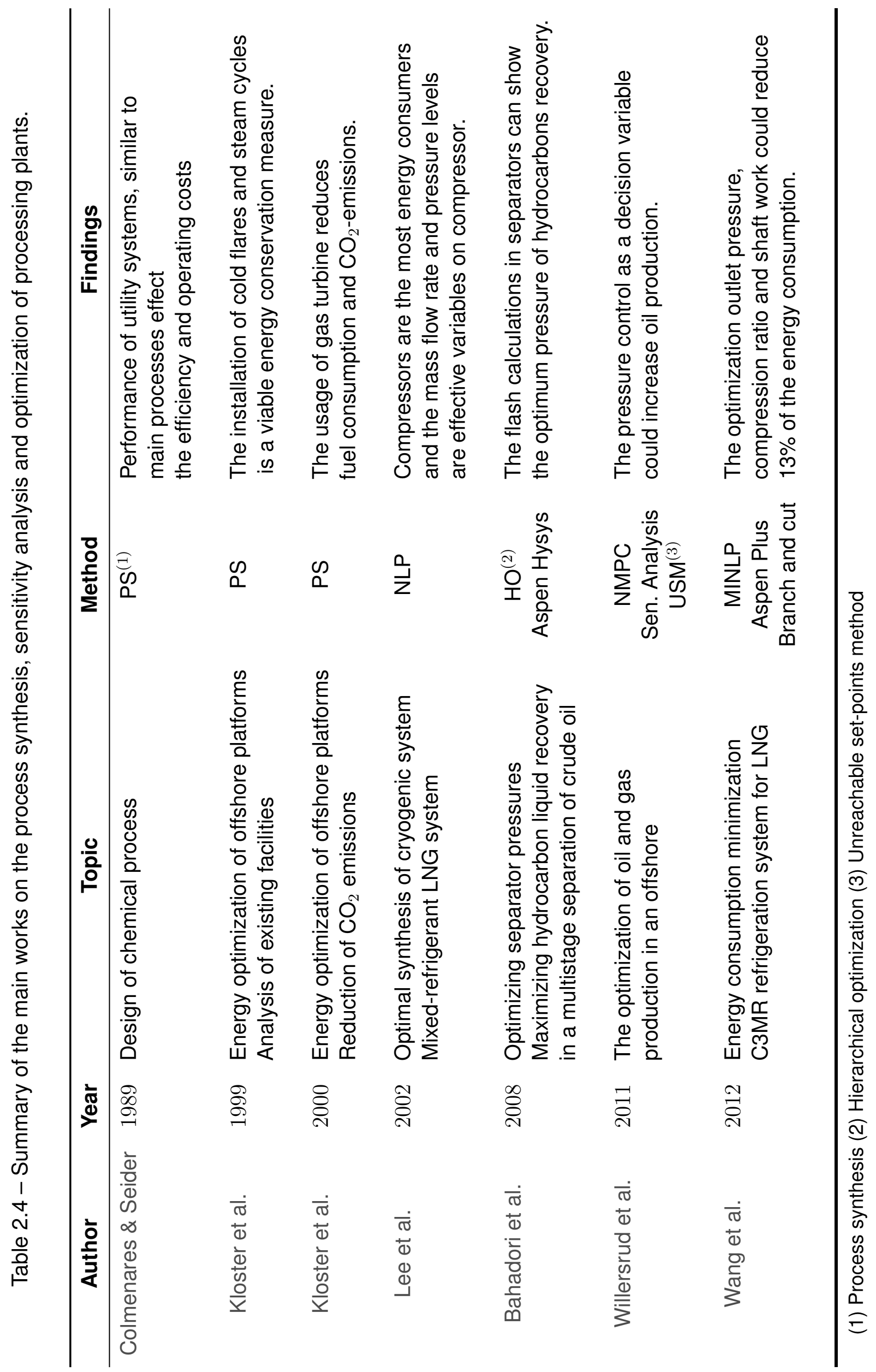




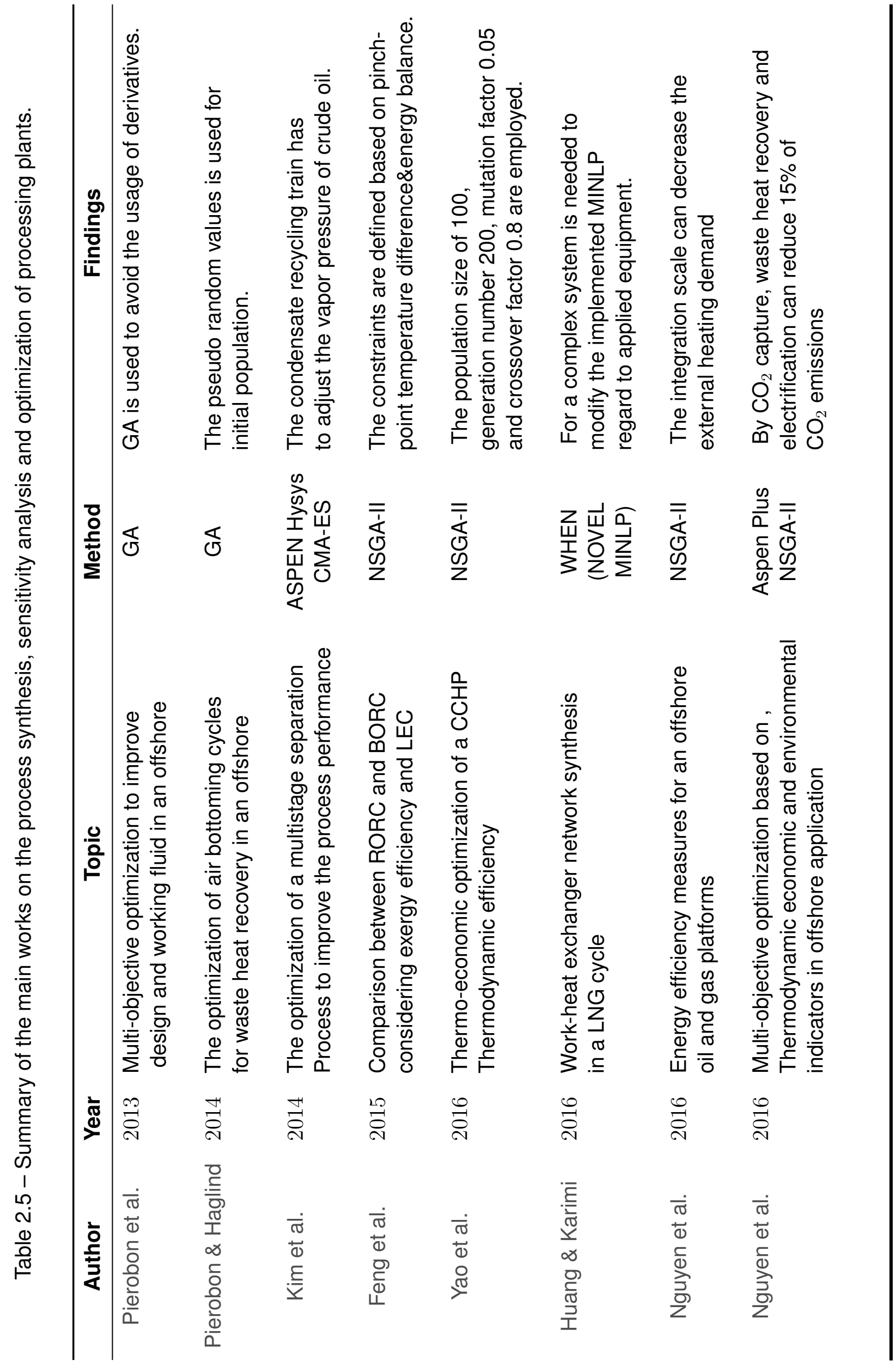




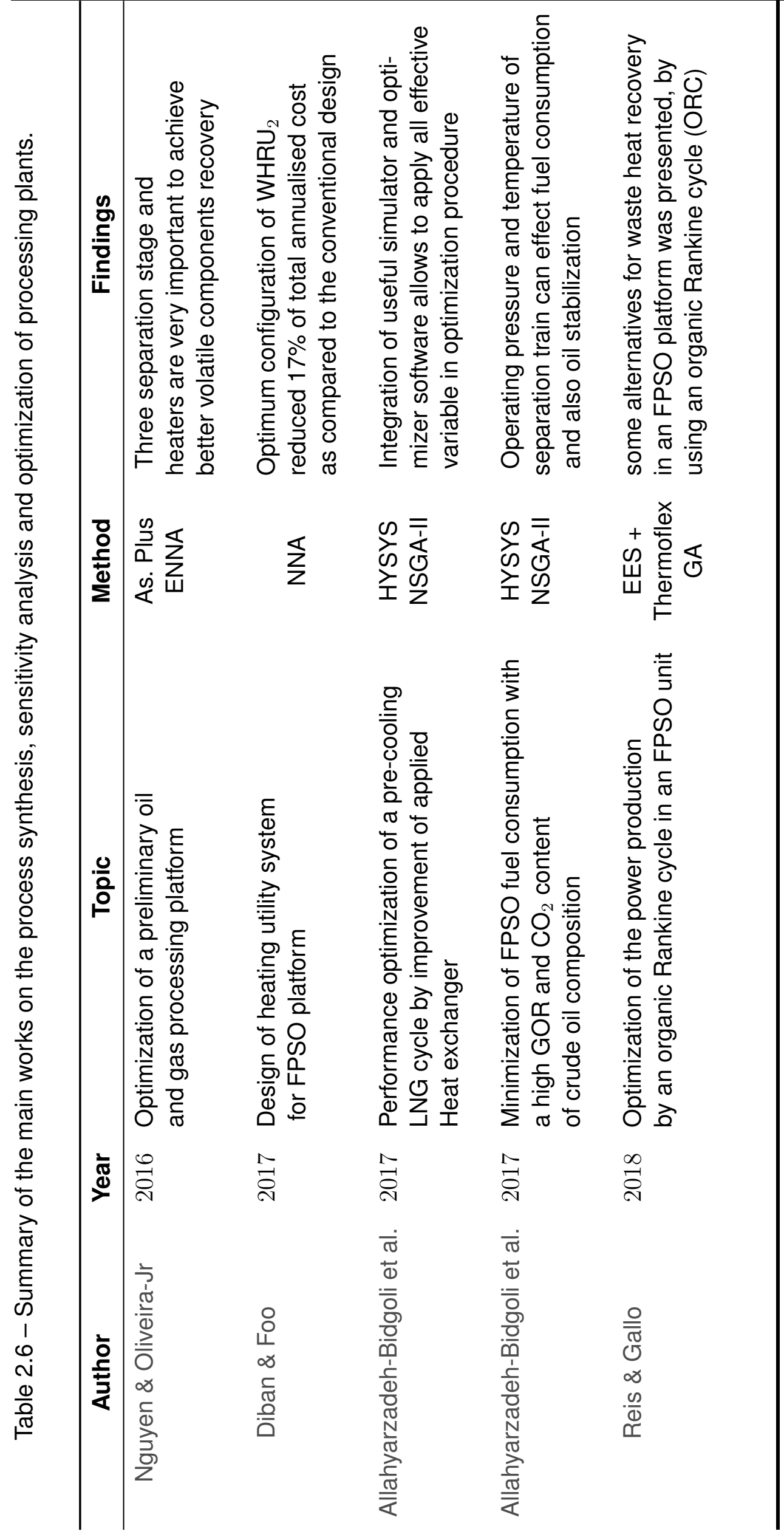




\subsection{Overview}

The literature review showed that for various reservoir fluids and operating conditions, there are different types of offshore installations operation. In addition, Aspen Hysys and Aspen Plus are the most used simulators for chemical processing, including oil and gas processing plants. These softwares are normally integrated with optimization softwares such as Matlab and Excel. However, ModeFrontier is the most comprehensive optimization software, but, from the literature review, there is not any coupling of Aspen Hysys or Aspen Plus with ModeFrontier.

On the other side, GA is the most chosen optimization method for the researches on oil and gas processing plants. This is because of the complexity of oil and gas processes, variety of applied equipment, number and types of existing design variables, and avoiding the calculation of derivatives in optimization.

Moreover, key operating parameters for performance of thermal systems and desired productions are operating pressures and temperatures of the separation process, heaters, compressors, and pumps. As shown in the literature review, these operation parameters are many, thus, to understand their effects on objectives and to reduce less effective parameters, a sensitivity analysis is necessary. However, in all works with a sensitivity analysis section, it is just a sensitivity of optimum solution that shows how the input parameters change the optimum results.

Finally, the literature review did not present any research discussing the possibilities for the improvement of a Brazilian FPSO plant configuration (in current operation) by changing thermodynamic parameters. Furthermore, none of the published researches addressed the potentiality of the optimization procedure to reduce fuel consumption and to improve the separation performance to enhance oil production and yield products with higher value.

In the next chapter, the theoretical foundations of thermodynamic analysis and optimization methods and their advantages/disadvantages will be discussed. 



\section{THEORETICAL FOUNDATION}

This chapter is structured in terms of theoretical and conceptual frameworks. It consists of concepts and, together with their definitions and references to relevant scholarly literature, the existing theories used for thermodynamic assessment, Optimization methods, such as statistical analysis (screening analysis) methods, optimization algorithms, and sensitivity of optimum.

\subsection{Thermodynamic Analysis}

The First Law of Thermodynamics states that energy can be neither created nor destroyed, but it only can be transformed from one form to another. Therefore, it gives indications on the processes in which energy is converted, lost and dissipated. For a control volume, in steady-state conditions and steady-flow processes, the energy balance is written as:

$$
\dot{Q}-\dot{W}=\sum \dot{m}_{\text {out }} h_{\text {out }}-\sum \dot{m}_{\text {in }} h_{\text {in }}
$$

where $\dot{Q}$ and $\dot{W}$ are heat rate and power, respectively, $\dot{m}$ is the mass flow rate of the material stream and $h$ is the specific enthalpy.

The mass balance and thermal efficiency of the applied gas turbine are calculated as follows:

$$
\begin{gathered}
\dot{m}_{\text {out_GT }}=\dot{m}_{\text {air }}+\dot{m}_{\text {Fuel }} \\
\eta=\frac{W_{\text {net }}}{\dot{m}_{\text {Fuel }} \times L H V}
\end{gathered}
$$

where $\dot{m}_{\text {out_GT }}$ is the output mass flow of the gas turbine, $\dot{m}_{\text {air }}$ is the input mass flow rate of the air in the combustion chamber, and $\dot{m}_{F u e l}$ is the consumed fuel mass flow in the combustion chamber. $\eta, W_{\text {net }}$ and $L H V$ are thermal efficiency, shaft power from the gas turbine and lower heating value, respectively.

The calculations of the physical (e.g. density) and thermodynamic properties of each substance require information, such as the pressure, volume and temperature $(P, \nu \& T)$. These properties are predicted using chemical thermodynamic models, which are based on either equations of state (EoS). Peng \& Robinson (1976) Equation of State (PR-EoS) is most commonly used EoS in offshore simulation works (GALLO et al., 2017; REIS; GALLO, 
2018). Since the equations of state for hydrocarbons such as C20+ are not directly available from the software component list, specific correlations were applied. Thus, the PR-EoS provides the calculation of the thermodynamic properties of $\mathrm{C} 20+$ according to its molecular weight and density. In addition, the prediction values of the liquid volume and density for $\mathrm{CO}_{2}$-rich fluids by PR-EoS are compared with some experimental works (SANTOS et al., 2017; WIESBERG et al., 2016; LUCAS et al., 2016). Results from PR-EoS were considered in a good agreement with the experimental data. The implementation of PR-EoS will be explained in the next chapter (subsection 4.3.2).

\subsubsection{Separation Performance}

One of the main purposes of any oil and gas processing plant is to maximize the recovery of each group of hydrocarbons. Light hydrocarbons, such as methane, ethane, and propane should be separated into the exported gas. Butane is dependent on the separation pressure (it can be gas or liquid) and pentane plus should be mixed into (exportation)the oil stream. Therefore, to perform the separation, according to three types of hydrocarbons, the separation efficiency is calculated as follows:

$$
\begin{gathered}
r_{\text {light }}=\frac{\sum_{C 1}^{C 4} \dot{n}_{i, \text { Separated_gas }}}{\sum_{C 1}^{C 4} \dot{n}_{i, f e e d}} \\
r_{\text {medium-heavy }}=\frac{\sum_{C 5+} \dot{n}_{i, \text { Separated_oil }}}{\sum_{C 5+} \dot{n}_{i, \text { feed }}}
\end{gathered}
$$

where $r_{\text {light }}$ and $r_{\text {medium-heavy }}$ are separation efficiency of light hydrocarbons and medium with heavy hydrocarbons, respectively, $\dot{n}_{i}$ is the molar flow, $C_{1}$ is methane, $C_{4}$ is butane, and $C_{5+}$ are pentane plus components.

Finally, the separation efficiency $\eta_{s e p}$ is given:

$$
\eta_{\text {sep }}=r_{\text {light }} \times r_{\text {medium-heavy }}
$$

\subsection{Optimization}

The process of designing systems is developed over the centuries to make more accurate predictions and better conception. The design of a system can be formulated as optimization problems in which a performance measure is optimized while all other requirements are satisfied. Many optimization methods have been developed and used to design better systems. 
An "optimization problem" is the problem of finding the best possible solution from feasible solutions space. Thevenin \& Janiga (2008) presents the optimization's meaning in the following words: " the design and operation of a system or process to make it as good as possible in some defined sense."

Mathematical optimization methods are created to allow recognizing the constraints and best suitable solution, but have not permeated all engineering purposes yet. As an engineer, the ultimate propose in conception, construction, and even maintenance of engineering system is to minimize the required attempts or to maximize the desired benefits. Since the effort required or the benefit desired can be mathematized as a function of certain decision variables and constraints in any practical situation, optimization can be defined as the process of finding the conditions that give the maximum or minimum value of a function (RAO, 2009).

The classical methods of optimization are applied to find the optimum solution of continuous and differentiable functions. These methods are analytical and the differential techniques that are used to locate the optimum points. For instant, consider $f(x)$ as one function variable that has a relative or local minimum at $x=x^{*}$ if $f\left(x^{*}\right) \leq f\left(x^{*}+h\right)$ for all sufficiently small positive and negative values of $h$. Likewise, a point $x^{*}$ is called a relative or local maximum if $f\left(x^{*}\right) \geq f\left(x^{*}+h\right)$ for all values of henough close to zero. On the other side, a function $f(x)$ has a global minimum at $x^{*}$ if $f\left(x^{*}\right) \leq f(x)$ for all $x$, and not just for all $x$ close to $x^{*}$, in the domain over which $f(x)$ is defined. Similarly, a point $x^{*}$ will be a global maximum of $f(x)$ if $f\left(x^{*}\right) \geq f(x)$ for all $x$ in the domain. Figure (3.1) shows the difference between the local and global optimum points and the value of $x=x^{*}$ is to be found in the interval $[a, b]$ such that $x^{*}$ minimizes $f(x)$.

Figure 3.1 - Relative and global minima.

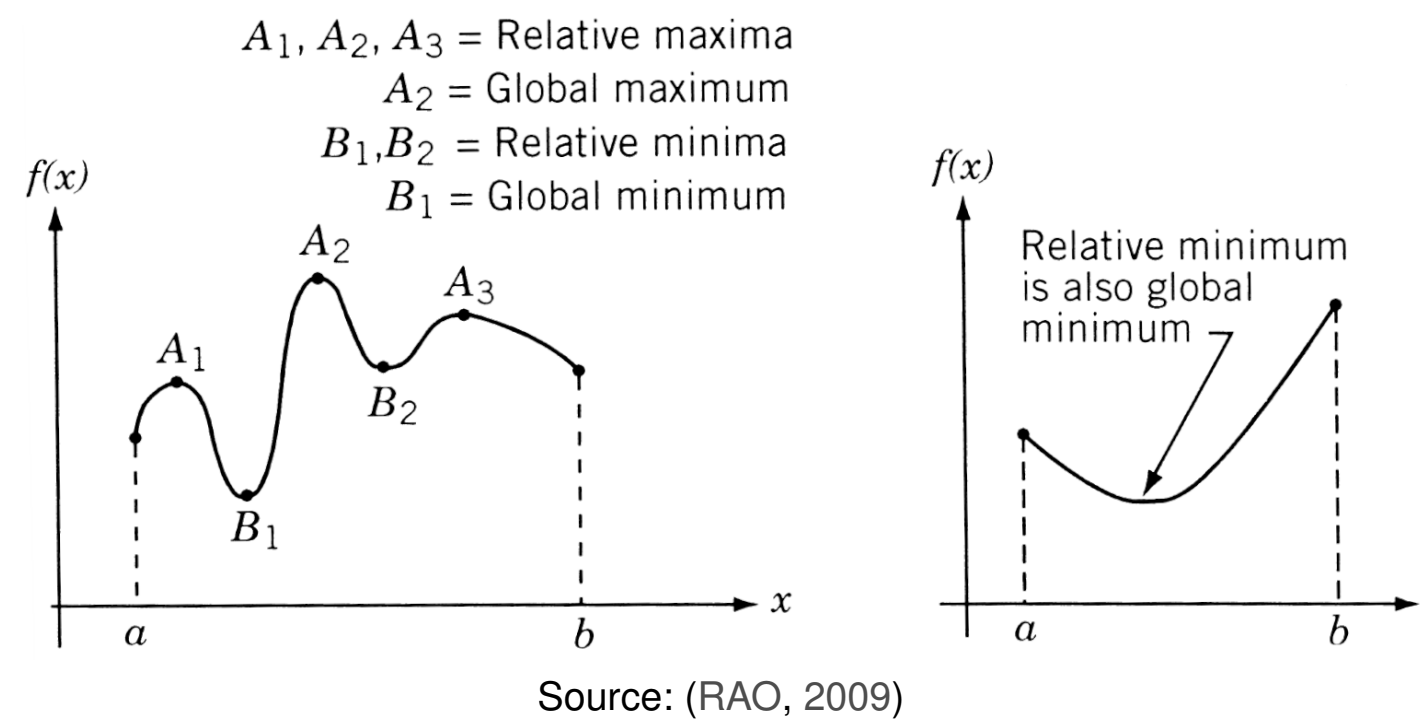


An optimization problem can involve the equality and inequality constraints. Thus, a mathematical programming problem or optimization procedure can be stated as follows:

$$
\text { Find } X=\left\{\begin{array}{l}
x_{1} \\
x_{2} \\
\vdots \\
x_{n}
\end{array}\right\} \text { which minimizes } f(X)
$$

and $f(x)=C=$ Constant

subject to the constraints

$$
\begin{gathered}
g_{j}(X) \leq 0, j=1,2,3, \ldots, m \\
g_{j}(X) \geq 0, j=1,2,3, \ldots, p \\
l_{j}(X)=0, j=1,2,3, \ldots, q
\end{gathered}
$$

where $X$ is an $\mathrm{n}$-dimensional vector called the design vector, each value of $C$ corresponds to a different member of a family of surfaces, $f(X)$ is the objective function, and $g_{j}(X)$ and $l_{j}(X)$ are known as inequality and equality constraints, respectively.Figure 3.2 shows a design space where the infeasible region is indicated by hatched lines.

Figure 3.2 - Contours of the objective function in the constraint surfaces in a design space .

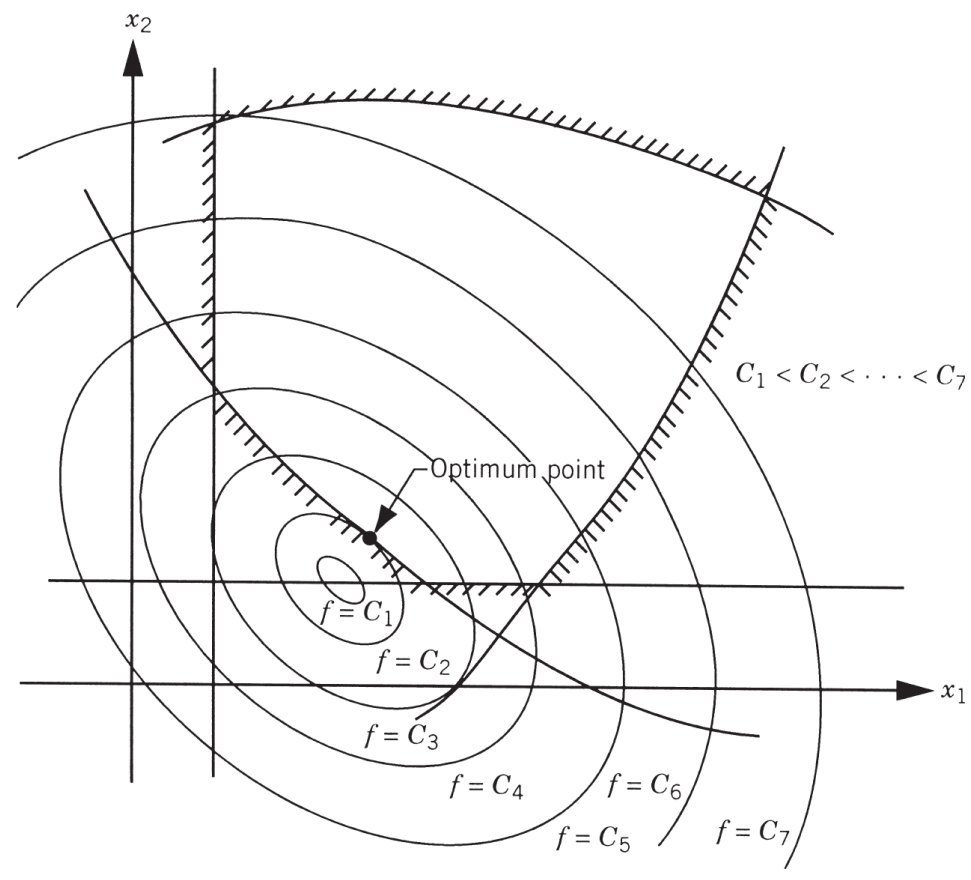

Source: (RAO, 2009)

Global optimization is distinguished from regular optimization by its focus on finding the maximum or minimum overall input values, as opposed to finding the local minima or maxima. Optimization problems can be divided into two major categories depending on whether the 
variables or functions are continuous or discrete, on whether direct and indirect methods are employed. As shown in Figure 3.3, the function can be continuous, continuous with discontinuity on the derivative, discontinuous and discrete or a combination of all of them.

Figure 3.3 - Input variable types in optimization.
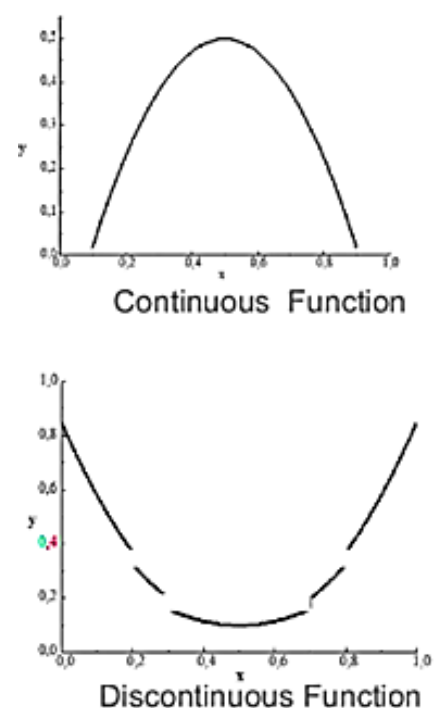

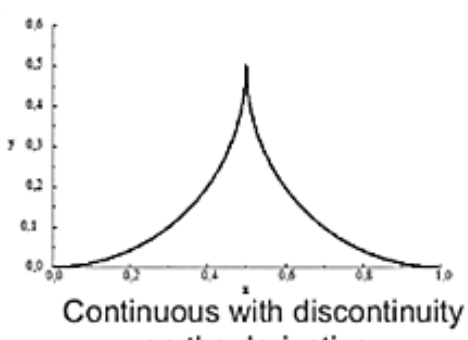
on the derivative

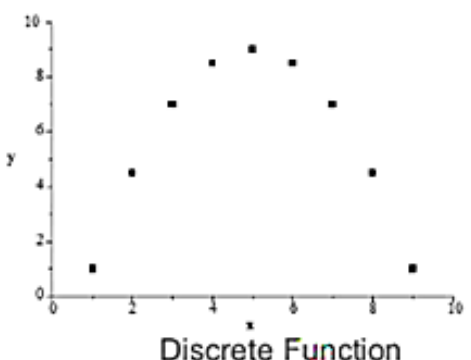

Source: www.esss.com.br

\subsubsection{Algorithmic methods}

Industrial Developments are dependent on computational abilities, including solving the problem in a systematic procedure. Formulating the synthesis of a processing flowsheet as an optimization problem is also proposed to be the main purpose of the algorithmic approach. Thus, it provides a more systematic framework to handle a variety of problems and very carefully accounting for features, such as interactions between components of a plant (GROSSMANN, 1985).

However, there is no guarantee that the converged solutions correspond to a global optima in the higher computational effort, but the optimal solution by algorithmic methods is only acceptable in terms of the alternatives that have been initially considered when building the search space. In many cases, to find a "better" local solution can be considered enough. In the worst case, unfeasible or unpractical solutions can be obtained (PAPOULIAS; GROSSMANN, 1983).

Even though, due to the advantages of the optimization of mixed integer nonlinear problems, the interest in the algorithmic methods is still rapidly increasing; it is widely chosen for the widespread utilization of commercial process simulators and modeling systems. The performance of algorithmic methods must be satisfactory for all reasonable choices of 
the initial variables. Therefore, reliable algorithmic methods could be robust and accurate, although the desired state uses less computer time and storage. On the other hand, it should be able to identify a solution without being overly sensitive to truncation or to round off errors (GROSSMANN, 2013; GONG, 2004).

\subsubsection{Classification of algorithmic optimization methods}

Since specific methods are available for the efficient solution of a particular class of problems, from a computational and methodological point of view, the classification of the optimization problems is important. At the first level, the numerical algorithms can be classified into mathematical programming (deterministic) and stochastic methods (SMITH, 2005). Mathematical programming requires the assessment of mathematical properties of the objective function and constraints, including objective function evaluations and calculation of gradients or even the second order of derivation, without involving any random steps. Deterministic methods can be further classified according to the type of problems that must deal with, e.g. linear or nonlinear (including sequential approaches, i.e., a successive solution of approximate sub problems), constrained or unconstrained, single or multi-variable problems, and discrete or continuous (or mixed) variable problems. The most traditional deterministic methods based on derivatives of the objective and constraint functions are the calculation methods, e.g. the Lagrange multipliers method. Elimination methods (dichotomous search) and pattern search methods (Nealder Mead simplex method, univariate search) are iterative techniques used to proceed from an initial guess towards the optimum without requiring the calculation of derivatives or making any assumption in the form of the objective function (HAFTKA; GÜRDAL, 2012).

Search methods are used for solving unconstrained non-linear problems and they can be divided into the zeroth (e.g. Powell conjugated directions), first (gradient-based) and second (Hessian-based) order methods. First and second order methods are based on calculating the first and second derivative of the function from a chosen starting point and then moving this point to the location.It causes the largest reduction in the objective function (if it is a minimization problem) following a step length criterion required by each method (HAFTKA; GÜRDAL, 2012).

For example, in the steepest descent first order method, the search direction is along the direction of the gradient of the objective function which is calculated from the current point. Gradient and Hessian matrices can be obtained numerically rather than analytically; therefore, multimodel functions could make the method unstable (JALURIA, 2007).

Among the methods are used for solving constrained optimization problems, more specifically those in which the objective function, as well as the constraints, are linear functions of the independent variables (linear programming), the simplex method is a widely used efficient 
scheme. This method searches along the boundary of the feasible domain from one vertex, given by the constraints, to the next until the optimum is obtained (JALURIA, 2007).

In the case of nonlinear constrained optimization problems, generalized reduced gradients and projected gradient methods, as well as the feasible direction methods, can be used. In these methods, the initial guess or starting point is in the feasible domain. Then, the search is moved to the constraint and obtains a point in the constraint. From this point, the search is moved tangentially to the constraint. Since by doing this, the trial point violates the constraint, the next step is used to bring the point back to the feasible region (JALURIA, 2007).

However, for the common, complex problems encountered in industrial and chemical plant flowsheets, those techniques are usually ineffective. Other approaches take advantage of the robust optimization tools, such as the mixed integer linear and non-linear programming, Mixed-integer linear programming (MILP), and Mixed integer nonlinear programming (MINLP) with Branch and Bound or Outer Approximation approaches that avoid the exhaustive evaluation of the integer variables by using relaxation models and a decision tree. Moreover, MIPSQP (Mixed Integer Programming Sequential Quadratic Programming) is a single-objective gradient-based optimization algorithm for solving non-linear mixed discrete-continuous problems. MIPSQP splits variables into relaxable (discrete ordered), non relaxable (discrete unordered) and continuous, and applies different strategies to each type. Therefore, the decision tree produces the partial and potential solutions through connection nodes. Nevertheless, these types of approaches still face two major challenges when are applied to process design: (a) existing non-convex models that conduct to local optima and (b) combinatorial explosion leading a difficulty to solve industrial processes (GROSSMANN, 1985; SMITH, 2005; INUTECH-GMBH, 2007; ANANTHARAMAN, 2011; XUE et al., 2012).

Thus, there has been considerable development in the use of optimization methods in process synthesis that include stochastic techniques and evolutionary algorithms, such as GA, which uses the random points from an initial population to orient the search direction or can take the search in a deterioration side of the objective function. This method can be useful and handle the problems when the calculation of the derivatives would be complex and make deterministic methods to fail (such as the objective functions of this research) (SMITH, 2005). The GA is detailed in the next subsection (subsection 3.2.5). Even though both mixed integer non-linear programming and genetic algorithms operate in a specified superstructure, genetic algorithms have the advantage of revealing more than one near-optimal configuration, so the designer may apply additional criteria to select the preferable one (FAZLOLLAHI et al., 2015). Another stochastic method one that is called global search metaheuristic method, uses the simulated annealing method, with an ability to find the global optimum from created the random search procedures. These random search processes evaluate the objective function at different points of the sample space that the points are chosen by a set of heuristics combined with the generation of random numbers (ANTONIN et al., 2005a). 
However, heuristics and genetic algorithm do not guarantee that the found optimum point is actuality a global optimum solution, since the optimum configuration may be one that is currently unknown. They also do not provide a common framework for synthesizing a variety of different types of major system components or even operating conditions, but there are several genetic algorithmic approaches that have been widely applied in process synthesis and optimization works. Hence, the genetic algorithm is selected for complex systems using the crossover and mutation operators very close to 1.0 which allow a broad capability of exploration and exploitation of the solution space.

For optimization problems, simulations can be approximated by optimizing metamodels as surrogates for the costly simulation response functions. Local metamodels can be used within an iterative optimization strategy, developed or updated as the optimization progresses. Its run times is generally shorter than the original simulation design and its response is deterministic (BARTON; MECKESHEIMER, 2006). On the other words, the metamodeling process includes basically four steps: (1) choosing a Design of Experience (3.2.4) or the manner to consistently conduct the (numerical) experiments and generate data; (2) selecting a model (of the simulation model) to represent the data; (3) fitting the model and; (4) validating the model from the observed data that are obtained in the first step. There are many selections for each of these steps and combination among them also can be considered to develop many metamodeling techniques. The most frequently used methods include response surface methodology (RSM), inductive learning, artificial neural network, and Kriging models.

\subsubsection{Screening Analysis}

Screening Analysis (sensitivity analysis) is a tool to evaluate the process structures and operational behaviors of chemical and industrial cycles. Thus, an analysis of sensitivity is to determine the rate at which the objective function changes while one of the decision variables is changed. Therefore, cost, power and energy consumption minimizations, and the performance, profits maximizations are important objectives in all design efforts. Despite several progress made in the last few decades, many challenging problems relating to oil and gas processing are still not addressed. In the current context, the sensitivity analysis methods are described and discussed.

\subsubsection{Design of Experiments (DoE)}

Design of Experiments (DoE) is a methodology that maximizes the knowledge gained from experimental data. It is originated in 1920 by a British scientist, Sir R. A. Fisher, (FISHER et al., 1937) to provide a strong tool to design and to analyze experiments.

The assumption of a random error exists in a laboratory experiment; it is fundamental and 
different between its stems. The classic use of DoE techniques assumes technical discipline in field experiments of some randomness and non-repeatability. The classic DoE methods, such as full-factorial design, central composite design (CCD), Box-Behnken, and D-optimal design (DOD) are developed for arranging laboratory experiments, with the consideration of reducing the effect of random error (GIUNTA et al., 2003).

The laboratory experiments (physical experiments) are not the only way to study physical processes. Nowadays, computational experiments are performed as an essential tool for any research areas and due to increasing and accessibility of computing potential, they are considered as conventional steps in design engineering.

The underlying model in a computer experiment is deterministic and given, but it is often very complicated to manage and analyze. One of the goals of computer experiments is to find an approximate model that is much simpler than the original one (FANG et al., 2005).

Similarly, modern DoE methods can also help to discover the key parameters which influence the objectives of design variables and the success of the optimization. A designed experiment is a purposeful sequence of assays. Before changing the input variables of a process, they are arranged in a way that allows identifying some observations, corresponding to changes in the output response (ALAGUMURTHI et al., 2006). DoE methodologies are used for different applications:

- To create sampling for screening analysis in a way to identify which input variables most affect the experiment.

- To create a set of stochastic points for robustness evaluation and reliability analysis.

-To generate an appropriate set of support points for response surface approximation.

- To provide the optimization algorithms with an initial population of designs.

Simply, It is possible to say:

The objective of using DoE is selecting the point where the response should be evaluated.

Modern DoE methods such as Latin Hypercube Sampling (LHS), Orthogonal Array Design (OAD), Uniform Design (UD), and Incremental Space Filler (ISF) were developed for deterministic computer experiments without the random error, as occurs in laboratory experiments (GIUNTA et al., 2003).

In this work, Latin Hypercube Sampling (LHS) and Incremental Space Filler (ISF) have been chosen as the DoE methods to generate samples randomly for identifying which input variables most affect the computer experiment, and to provide an initial population of designs for the optimization algorithm. Their details will be presented as follows. 
3.2.4.1 Latin hypercube sampling (LHS), Uniform Latin Hypercube (ULH) and Incremental Space Filler (ISF)

Latin hypercube design (MCKAY et al., 2000), can be viewed as an N-dimensional extension of the traditional Latin square design (MONTGOMERY, 1997). It is a type of stratified sampling that can be applied to multiple variables. In other words, it is a method for ensuring that each probability distribution in its model is evenly sampled. The concept behind LHS is not overly complex. Variables are sampled using an even sampling method, and then randomly combined sets of those variables are used for one calculation of the target function. It thus works by controlling the way in which random samples are generated for a probability distribution.For instance, in two-dimensional design (Latin square), the property is equivalent to each row and each column containing exactly one design point. If a regular grid sampling is considered, Latin hypercubes are constructed to avoid the collapsing property of grids: No two LH design points share the same value for any parameters (Figure 3.4) (URBAN; FRICKER, 2010).

Figure 3.4-Example of the regular grid (left) and Latin square (right) designs for two-dimensional design with 9 member ensemble.

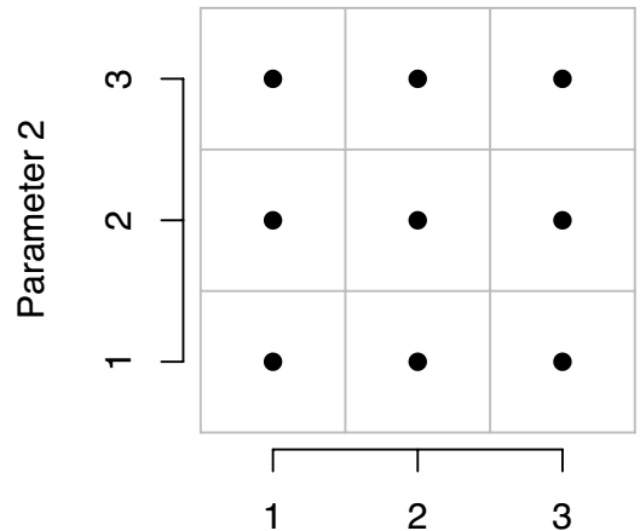

Parameter 1

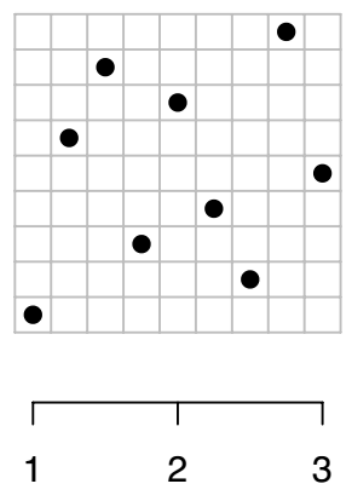

Parameter 1

Source: Urban \& Fricker (2010)

For a same size of $\mathrm{N}_{s}$, that can be constructed by dividing the range of each input variable into $\mathrm{N}_{s}$ strata of equal marginal probability $1 / \mathrm{N}_{s}$ and sampling once from each stratum.

While LHS represents an improvement over unconstrained stratified sampling, it can provide sampling plans with very different performance in terms of uniformity measured. Figure 3.5 illustrates this shortcoming which the LHS plan in Figure 3.5 (c) is significantly better than that in Figure 3.5 (a). 
Figure 3.5 - LHS designs with significant differences in terms of uniformity.

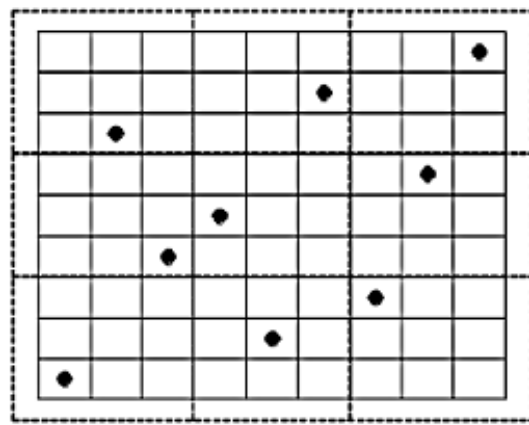

(a)

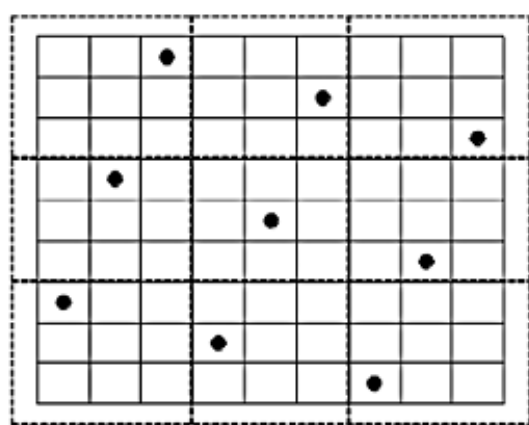

(b)

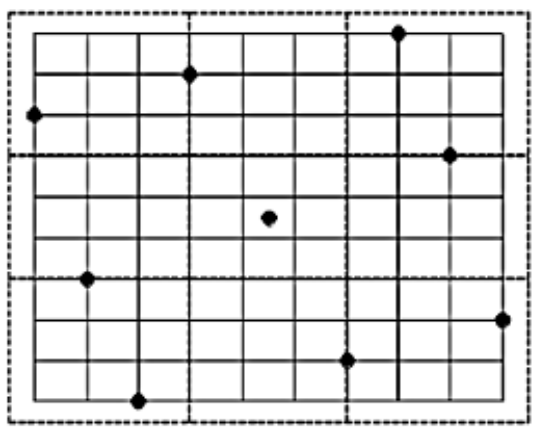

(c)

Source: Leary et al. (2003)

The advantage of the Latin hypercube sample appears when the output is dominated by a few of input components. This method makes sure that each of those components is represented in a fully stratified manner, no matter which components might turn out to be important. The $\mathrm{N}$ intervals in the range of each input component are combined to form $N^{K}$ cells, which cover the sample space of input variables. These cells, which are labeled by coordinates corresponding to the intervals, are used when finding the properties of the sampling plan (MCKAY et al., 2000).

In addition, this design has all locations lying along a single line and has no data points in the remainder of the design space. Hence, a secondary criterion is used to ensure that the design selected does indeed have good space-filling properties. Two common choices for ensuring good space-filling and for an LHS (or other possible design construction strategies) are (MYERS et al., 2016):

Minimax: A minimax design is one that minimizes the maximum distance between any location in the design space and its nearest design point. This criterion directly targets the objective of allowing a design point to never be too far away from a new location where it can be predictable.

Maximin: A maximin design is one that maximizes the minimum distance between any two design points. This criterion forces neighboring points to be as far apart as possible, thereby filling the space. It also has the advantage of allowing comparing distances just to involve the design points, instead of all the possible locations in the design space.

The Uniform Latin Hypercube (ULH) It is another type of $\mathrm{LH}$ that generates random numbers regarding a Uniform Distribution. It is a stochastic DoE algorithm which generates the random points are conforming to a Uniform Distribution and has particular adjustments for optimization by genetic algorithms and RSM. ULH is an advanced version of Monte Carlo Random Sampling with more precisely in constrained Monte Carlo (i.e. random) sampling 
scheme. The constraint refers to the way each variable is sampled: the uniform statistical distribution is splitted into $n$ intervals with the same probability, and then a random value is selected within each interval. Therefore, the points are relatively uniformly distributed over the variable range (Esteco SPA, 2017). In addition, even though the Latin Hypercube generally gives correct distributions only in case of continuous input variables (i.e. bases equal to zero), ULH is able to generate proper uniform distributions in case of discrete variables.

Incremental Space Filler (ISF) It is an augmenting algorithm which adds the new design to existing points in the database (previously generated designs). It can add new points in order to fill the space in a uniform way with maximizing the minimum distance from the existing points as shown in Figure 3.6. It is normally recommended to avoid generating the initial database with a suitable DoE for statistical analysis, but rather with another space filler such as ULH. One of the useful distribution of ISF is when it algorithm type samples according to Genetic Algorithm. Thus, the maxmin criterion is optimized with a genetic algorithm, which is rather a fast and robust method.

Figure 3.6 - Incremental Space Filler (ISF). Existing points in the database (previously generated designs) (a), New points are added to fill the space uniformly (b).

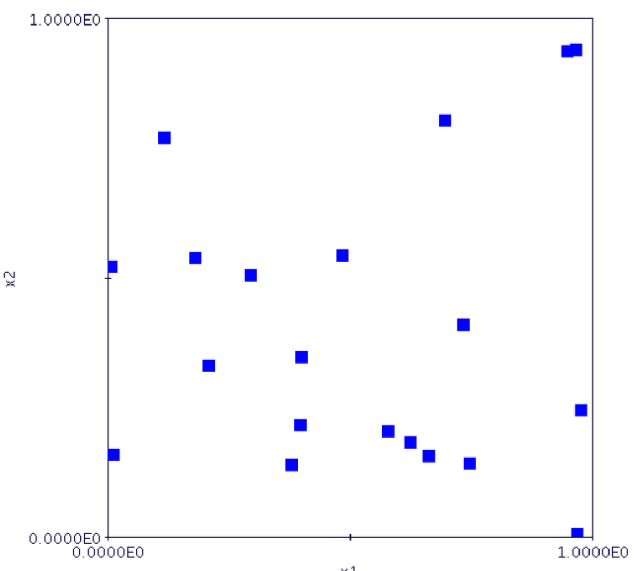

(a)

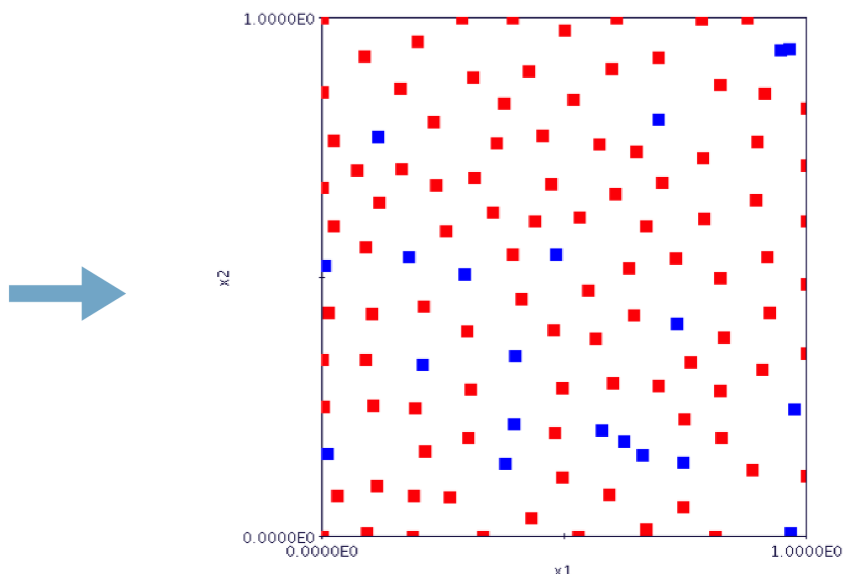

(b)

Source: ESTECO WEBINAR SERIES (2016)

\subsubsection{Smoothing spline ANOVA method (SS-ANOVA)}

Analysis of variance (ANOVA) is a set of statistical assumption and their related procedure are used to analyze the difference between group averages (ANSCOMBE, 1948). Borgonovo (2017) in Sensitivity Analysis book and in the importance of ANOVA mentioned that:

"The functional ANOVA expansion of a multivariate mapping is a fundamental result in statistics." 
It originated from the work of Fisher \& Mackenzie (1923) and Hoeffding (1948), and has been firmly established in Efron \& Stein (1981). The importance of this result in statistical models is evidenced by the several alternative proofs available in the literature(SOBOL; KHAARA, 1969; SOBOL, 1993; OWEN, 2003).

To analyze different effects or elements in ANOVA, i.e., main effects and interaction effects, Kherad-Pajouh \& Renaud (2010) advocated a sequential approach: first assigned the section of the explained variance to the main effects (one after another), then to the interactions (two-way) and then to an increasing extent higher-order interactions, if present. However, in a case with a set error according to a Gaussian distribution, it does not likely hold in many applied datasets. In that, the necessary conditions for a parametric ANOVA analysis are not satisfied, at least as a check for the parametric test.

In the current context, the Smoothing Spline ANOVA (SS-ANOVA) model is implemented for the analysis of variance. It can be used for both univariate and multivariate statistical modeling. This method is a statistical modeling algorithm based on functional decomposition similar to the classical analysis of variance (ANOVA) decomposition and is associated with notions of the main and interaction effects. For these reason, the interpretability of the results is an important additional benefit over standard parametric models. The selected parameters are the inputs in which their variations can cause variance in the responses (outputs) (WAHBA, 1978; WAHBA et al., 1995; GU, 2013).

The framework for SS-ANOVA is a general multiple nonparametric regression model with $\mathrm{d}$ independent variables $\left(x_{1}, x_{2}, \ldots \ldots, x_{d}\right)$, continuous or discrete, and response variable $y_{i}$ (RICCO et al., 2013).

$$
y_{i}=f\left(x_{1 i}, x_{2 i}, \ldots, x_{d i}\right)+\varepsilon_{i}, i=1,2, . ., n
$$

Through the SS-ANOVA decomposition, an unknown mean function is decomposed as a sum of the main effects $\left(f_{k}\left(x_{k}\right)\right)$ and interaction effects $\left(f_{i, j}\left(x_{i}, x_{j}\right)\right)$. For example:

$$
E\left(x_{1}, x_{2}, \ldots, x_{d}\right)=f\left(x_{1}, x_{2}, \ldots, x_{d}\right)=\mu+\sum_{k=1}^{d} f_{k}\left(x_{k}\right)+\sum_{i<j} f_{i j}\left(x_{i}, x_{j}\right)+\ldots
$$

SS-ANOVA is an extremely flexible class of additive models which allows selecting a parsimonious model from a large class of non-parametric additive models. It can also include linear terms for discrete variables (equivalent to ordinary ANOVA), linear or smooth terms for continuous variables, and interaction terms between continuous and discrete variables.

There is a practical aspect of suing with high dimensional space that is necessary to take into account. This unpleasant reality that is also called curse of dimensionality, affects every different side of multivariate analysis and is an unavoidable thing with the effects of the 
sparsity of the space. On the other words, when the number of input variables(dimensionality) increases, the volume of the space also grows up very fast and the available data scatters: in order to obtain a statistically significant and reliable result, the amount of needed sampling data increases exponentially with the dimensionality.

A major consequence of dimensionality on SS-ANOVA model has a very fast growing in the number of parameters (degrees of freedom) that would be required by the introduction in the model of higher-order effects in a high-dimensional space. So considering only main effects in building the model-or possibly adding at most only interaction effects-helps in tackling the curse of dimensionality. If $N$ is the number of input variables, the number of main effects terms is clearly equal to $N$, while the number of interaction effects is equal to $N(N-1) / 2$, so the growth rate of second order models goes as $O\left(N^{2}\right)$. For this reason, in practical data analysis in a high-dimensional space, usually, only the main effects are included. Interaction effects are taken into account only if the relevant computational demand is affordable.

A useful diagnostics tool for assessing the model quality is represented by the collinearity indices, $\kappa_{k}$ (RIGONI; RICCO, 2011). Defining the pxp cosines matrix $\mathrm{C}$ as

$$
C_{i j}=\frac{\left(\Gamma_{i}^{*}, \Gamma_{j}^{*}\right)}{\left\|\Gamma_{i}^{*}\right\| \cdot\left\|\Gamma_{j}^{*}\right\|}
$$

Finally, the diagonal elements of $C_{k k}^{-1}, \kappa_{k}$, is

$$
\kappa_{k}=\sqrt{C_{k k}^{-1}}
$$

The ideal situation of all $\kappa_{k} \approx 1$ holds only in the case that all the $\Gamma_{i}^{*}$ are nearly orthogonal to each other. In case of two or more $\Gamma_{i}^{*}$ are highly (linearly) correlated - a phenomenon referred to as concurvity (or as identifiability problem) - can be detected since the relevant collinearity indices will be much greater than unity. This unfortunate situation occurs when the chosen model decomposition is inadequately supported on the sampling points domain. There can be many causes to this pathology: dependent input variables, bad sampling points, too low sample points, etc (DEZAN, 2015).

Beside of the collinearity indices indicator, there is another statistical chart tool that called Scatter matrix chart, to show the measure of association between pairs of selected (input) variables computed using the Pearson correlation. It has an additional scatter chart for each variable pairs and Probability Density Function charts for each single variable (on the main diagonal) to check any linear dependence (correlation) between two variables and the nature and the strength of such correlation. The application detail of this tool is expressed in the next chapter (subsection 4.3.3). 


\subsubsection{Genetic Algorithms - Fundamentals}

Genetic Algorithm (GA) is a direct, parallel, stochastic method of optimization, which is based on Darwin's theory of natural selection. GA belong to the class of Evolutionary Algorithms (EA). Evolutionary algorithms use the three main principles of natural evolution: reproduction, natural selection (crossover) and diversity of the species (mutation), accomplished by the differences of each generation from the previous one. Genetic Algorithms generate a set of individuals, representing possible solutions of the design (population) as initial population. The crossover is implemented by using a criterion, giving an evaluation for the individual regarding the desired solution. The best-suited individuals create the next generation. The large variety of problems in the engineering, as well as in other fields, needs the use of algorithms from different types, characteristics and settings such GAs(ARORA, 2016).

The main ingredients of GA are divided into major parts:

- Chromosomes:

For the genetic algorithms, the chromosomes act as a set of genes, which code the independent variables. Every chromosome represents a solution of the given problem. Individual and vector of variables will be used as other words for chromosomes. A set of different chromosomes (individuals) forms a generation (Figure 3.7). By means of evolutionary operators, such as selection, recombination and mutation an offspring population is created (POPOV, 2005).

Figure 3.7 - The Genes, chromosomes and genetic operations.

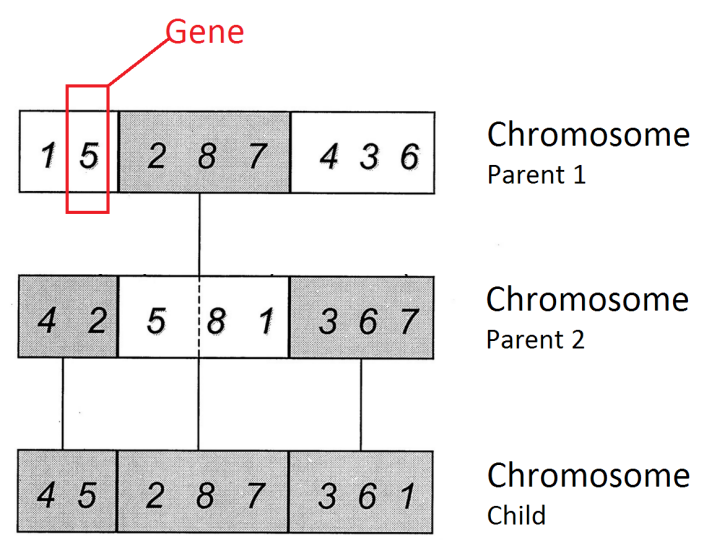

Source: https://protosity.wordpress.com/ 


\section{- Selection:}

In EA the selection of the best individuals is based upon an evaluation of the fitness function or fitness functions. Examples for such fitness function are the sum of the square error between the wanted system response and the real one; the distance from the poles of the closed-loop system to the desired poles, etc. If the optimization problem is a minimization one, individuals with a small value of the fitness function will have greater chances for recombination and, respectively for generating offspring (POPOV, 2005).

- Recombination:

The first step in the reproduction process is recombination (crossover). In it, the genes of the parent areas are used to form an entirely new chromosome. The GA's recombination is an operation seeking two parents, however plan (design) with more parents area can also possible. Two of the most widely used algorithms are Scattered Crossover and Blending Crossover (OYAMA, 2000).

After Recombination, the crossover operator changes the combination of the offspring by exchanging part of the parents strings and hence creates new strings. Crossover is also created stochastically by a suitable crossover probability. The mutation is necessary to generate a point in the vicinity of the current point, thereby performing a local search around the current solution that is not normally possible by reproduction and crossover. Mutation increases the variability of the population. In a binary alphabet of GA for representing a chromosome, mutation provides variation in the population by altering a bit of the string from 0 to 1 or vice versa with small mutation probability (MISHRA et al., 2009).

In the genetic algorithm method, populations of strings (the analogy of chromosomes) are created to represent a set of parameters (e.g. temperature, pressure or concentration), so that basic elements of natural genetics (i.e., reproduction, crossover and mutation) can be used in the genetic search procedure. Thus, differently from deterministic approaches, genetic algorithms move from one set of points (population) to another set of points. Other stochastic methods include the simulated annealing method, which has the ability to find the global optimum due to the random search procedures that only evaluate the objective function at different points of the search space (FRANGOPOULOS et al., 2002). The points are chosen by using a set of heuristics combined with generation of random numbers; that is why they are also called global search metaheuristic methods (ANTONIN et al., 2005b) .

\subsubsection{Non-dominated sorting genetic algorithm II (NSGA-II)}

The Non-dominated Sorting Genetic Algorithm II (NSGA-II) was developed by Prof. Deb et al. (2002) at the Kanpur Genetic Algorithms Laboratory (KanGAL). In NSGA-II, the first parent population and the offspring population created by parents using genetic operators, are combined to form a new population. Then, the points of the new population are sorted in 
different non-dominated fronts, according to their level of non-dominance. After that, the new parent population is created by points of the fronts. Slots in the new population are filled up starting with the first front, the second one and so on (DEB et al., 2002).

NSGA-II is a fast and elitist multi-objective evolutionary algorithm. Its main features are (Esteco SPA, 2017):

- A fast non-dominated sorting procedure is implemented. Regarding the level of non-domination, sorting the individuals of a given population is a complex task, thus, non-dominated sorting algorithms are generally computationally expensive for a given large population sizes. Hence, the solution adopted carries out an intelligent sorting strategy.

- NSGA-II applies the elitism for objective search, using an elitism-preserving approach. Elitism stores all non-dominated solutions discovered so far, beginning from the initial population and enhances the convergence properties.

- Since NSGA-II adopts a suitable parameter-less niching approach, diversity and spread of solutions are guaranteed without using sharing parameters. Moreover, the applied crowding distance, guides the selection process towards a uniformly spread Pareto frontier.

- NSGA-II allows both continuous and discrete design variables.

\subsubsection{Hybrid optimization method (GA+SQP)}

Hybrid method is an optimization algorithm combining a steady-state genetic algorithm with a Sequential Quadratic Programming (SQP) optimizer (in the ModeFrontier). The SQP algorithm does not merely perform a local refinement around the quasi-optimal designs found by the genetic algorithm. On the contrary, the two algorithms cooperate and exchange information in several ways during the entire run. Hybrid is suitable for solving both single and multi-objective problems. The genetic algorithm implemented in Hybrid promotes an efficient use of computational resources by combining the steady-state evolution with a controlled elitism procedure. The designs are ranked by applying the non-dominated sorting and crowding distance methods. The initial population size of Hybrid corresponds to the size of the DOE dataset. However, the population can grow up to two times the size of the initial population, but it cannot shrink. Hybrid performs a periodic ranking of the evaluated designs each time it receives the number of designs corresponding to the size of the previous population. If the number of points lying on the first Pareto front is larger than the number of points of the previous generation, all those points become the next parent population. If the number of points lying on the first Pareto front is smaller than the number of points of the previous population, all first front points are included in the next parent population plus the points taken from other fronts in a geometrically decreasing percentage (controlled elitism). Furthermore, Hybrid also performs an on-the-fly update of the parent population. As soon as a design is evaluated, Hybrid checks whether it is dominated by the parents: if this is 
not the case, it becomes immediately part of the parent population. The SQP algorithm is inserted in the run of the genetic algorithm as an extra operator, in addition to the classic GA operators (mutation and crossover). SQP runs, therefore, in parallel with the genetic algorithm and the user can decide the percentage of designs generated by this algorithm in the total number of designs. However, SQP is a sequential algorithm, so it performs only one evaluation at a time. All designs generated by SQP are added to those generated by the genetic algorithm, and sorted and ranked at each periodic update. The SQP algorithm starts from a single randomly selected non-dominated design of the GA parent population, which thus enhances its search efficiency.

\subsubsection{Sensitivity of the optimum}

The industrial processes are often subjected to variable environmental conditions. Thus, the system must be able to operate with variable feedstock and among other concerns. Thus, a process found to be optimum for certain conditions could not be reliable when operating under different specifications. Accordingly, an analysis of sensitivity of the optimum is recommended to determine the rate at which the objective function changes while one of the decision variables is changed. Such perturbations may also show the sensitiveness of the objective function, allowing determining the required control precision or the penalty for failure to control the variable within the imposed restrictions. The selection of the adequate design variables and objective/constraint functions is important for the success of the optimization process. As the number of independent variables increases, the computational effort for solving the optimization problem increases substantially, particularly for thermal systems, because of their complicated non- linear characteristics (BIEGLER et al., 1997).

Therefore, it is important to focus only on the dominant variables rather than considering all that might affect the solution. Additionally, a performed sensibility analysis considering constraints could indicate that which flexible constraint would be advantageous for reducing/ increasing the value of the objective function. Finally, it must be noticed that, even though a single component optimization can be applied in a preliminary optimization stage to some important components that dominate the cost picture, conditions that optimize a single component dose not usually optimize the total plant (GROSSMANN, 1985) (LAZZARETTO; TSATSARONIS, 2006).

\subsubsection{Conclusion}

Aspen HYSYS as one of the most popular process simulation softwares and preferred for applications related to the oil and gas industry is chosen to simulate all operating conditions and crude oil compositions with an adequate EoS in this work.

The PR-EoS is the most common used EoS in offshore simulation works and its predictions 
of the liquid volume and density for $\mathrm{CO} 2$-rich fluids are compared to some experimental works. According to the existing literature, the results from PR-EoS are in a good agreement with the experimental data. Hence, it is chosen as EoS of the current research.

Moreover, in order to obtain more precise results, using the real data of offshore is essential. Therefore, beside utilizing operating scenarios of a Brazilian FPSO that works on a pre-salt field (from early life to end of life of the reservoir), a real performance data of an aero-derivative gas turbine (RB211G62 DLE 60Hz turbine) with offshore application (as principal utility system of plant) from GATECYCLE ${ }^{\text {TM }}$ commercial software (General Electric , 2013) is applied here.

Furthermore, regarding the quantity of existing operating parameters as variables to evaluate the objectives, it is necessary to create an automated process (integration) to couple a simulator-modeler software with a statistical analyzer-optimizer tool to cut the development time between simulation models and outputs. ESTECO ModeFRONTIER ${ }^{\mathrm{TM}}$ is a powerful integration platform for a multi-objective and multi-disciplinary optimization that enables the automation of the design simulation process and facilitates analytic decision making. In the current thesis, for the first time, Aspen HYSYS is coupled with ModeFRONTIER to make an automated optimization process.

On the other hand, the complexity of the calculating derivatives of objective functions for this research can cause deterministic methods to fail. Therefore, the chosen method should be well suited for practical optimization problems, even difficult black-box problems possibly with discrete decision variables. Non-dominated Sorting Genetic Algorithm II (NSGA-II) with Controlled Elitism as a stochastic method and one of the most used evolutionary method is selected to be implemented here. Moreover, GA is a robust optimization algorithm that has the advantage of avoiding stalling problem, since it is able to find the global maximum/minimum in a problem with multiple local optima. Although, the existing hybrid methods based on NSGA-II will be verified in terms of convergence. The process synthesis based on evolutionary method needs stochastic techniques using random choice to guide the search. Therefore, the entire DOE table is used as a sequence of initial points for different local optimization problems and screening analysis that will be provided by the Uniform Latin Hypercube (ULH) DoE algorithm and Incremental Space Filler (ISF) (according to collinearity induces and Probability Density Pearson (PDF)) by the ESTECO ModeFRONTIER ${ }^{\mathrm{TM}}$ software.

The details and algorithm of the problem formulation for Aspen Hysys 9.0 and ESTECO ModeFRONTIER ${ }^{\mathrm{TM}} 2017$ and their coupling in the optimization process will be shown in the next chapter. 



\section{METHODOLOGY IMPLEMENTATION}

The description of the implemented of mathematical modeling and methodologies used in the current thesis is presented in this chapter.

\subsection{System description and simulation}

The Floating, Production, Storage and Offloading (FPSO) unit is an offshore installation used for petroleum productions and operations. Basically, FPSO uses the same equipment of a fixed installation. However, the great advantage of FPSO is that it can be used for operation in remote regions and its storage capability for the processed crude oil. Figure 4.1 shows the schematic of a typical FPSO unit.

Figure 4.1 - Top view of a typical FPSO unit and all related processes.

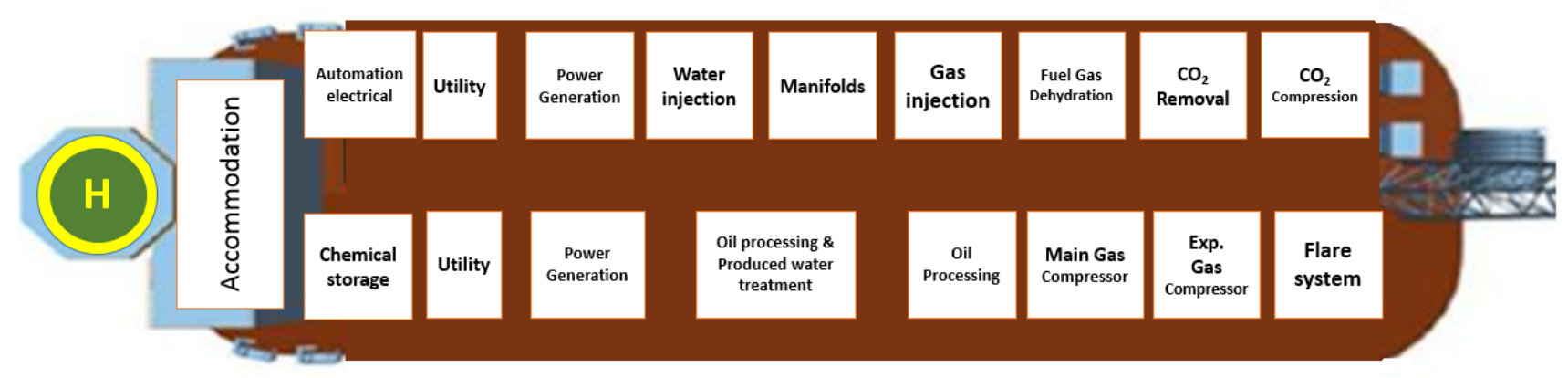

Source: Author

The process configuration and floating type of an offshore facility depend on the properties of the fluids and geological characteristics. The number of stages in the multistage separation train depends on reservoir compositions, GOR, the operating pressure, $\mathrm{CO}_{2}$ and salt content of crude oil. Accordingly, high API gravity oil fluid with high GOR operating under high pressure (200- 700 psi or $20-200$ psi) requires three to four separation stages. A four-stage gas and oil separation train is not usually economically attractive, because the recovery of oil increases only by $8 \%$ by adding one more stage (ABDEL-AAL et al., 2015). Therefore, because of the limitation of the space for an FPSO installation with GOR variation, three sequential phase separators are normally used for crude oil processing.

Operating conditions are often determined by the features of the fluid reservoir, based on the composition of the hydrocarbons and the amount of impurities in the oil content. Both 
pressure and temperature in multistage crude oil separation processes play an important role in improvement of the separation performance, specifically in the recovery of volatile components.

Figure 4.2 shows the combination among main and utility plants in a standardized FPSO used in pre-salt field.

Figure 4.2 - The general scheme of a FPSO

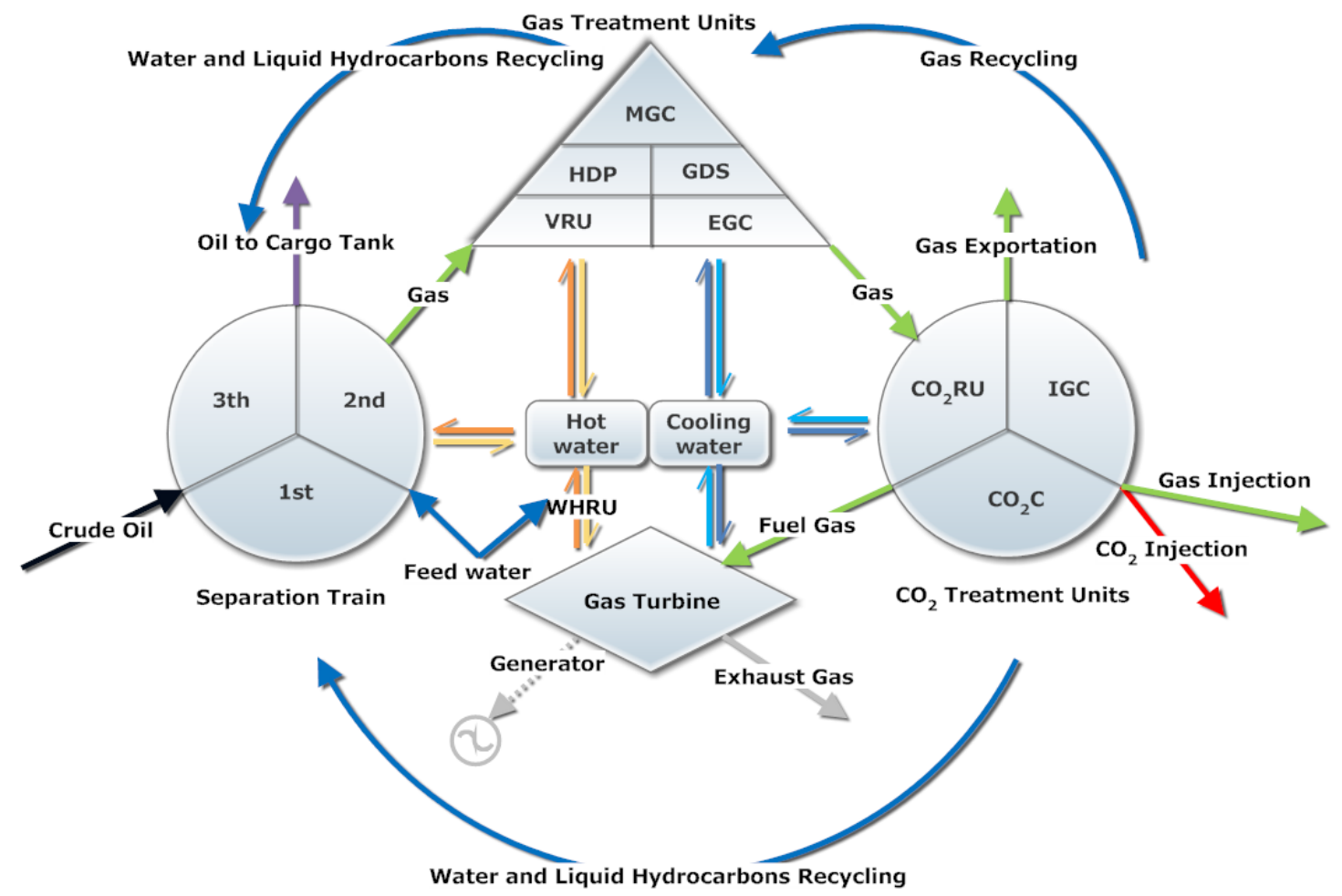

Source: Author

The simulation description of proposed FPSO with all related processes are explained in the following subsections:

\subsubsection{Separation Train}

Separation train is the main processing stage in an offshore installation, so that the separation of production from reservoir fluid occurs in this unit.

A three-stage separation train including oil pre-heating, oil heating, degassers and electrostatic treatment was simulated as shown in Figure 4.3. A free-water knockout (FWKO) separator type is used and it is responsible for separating associated gas and water from crude oil in the first stage of the separation train, with a processing capacity of 150,000 barrels per day, 
at the nominal pressure range of $2000 \mathrm{kPa}$ and temperature range of $20-30^{\circ} \mathrm{C}$. As shown in Figure 4.3, the crude oil from the well(s) enters the production manifold. Then, the oil, gas and water are separated by a three-phase separator (gravitational separator) at the first stage of separation. The separated gas in the first stage is sent directly to the MGC compressors and the separated water is forwarded to water treatment unit or water injection system.

At each stage, the operating pressures and temperatures are adjusted by valves and oil heaters, respectively. Thus, the pressure of the separated oil at the end of the first stage decreases by the first pressure valve and then, its temperature increases by pre-heating and finally, by heating (heat exchangers). In pre-heater, the heating of hot fluid (of this heat exchanger) is recovered by the oil stream (as exportation oil) leaving the processing plant. The hot water (hot fluid of heater) is provided by the WHRU and the oil is heated up to about $90^{\circ} \mathrm{C}$ by the heater (Figure 4.3).

In the second stage of separation, there is a pair of separator tanks called degassers and electrostatic coalescers. Degassers provide the separations of light and medium hydrocarbon fractions in operating pressures of about $800 \mathrm{kPa}$, and the output oil from that separator is forwarded to the electrostatic coalescer device (Figure 4.4). The gas separated at this stage is routed to the second stage of the VRU to be compressed. At this step, water drops remain separate from the oil by electric polarized plates and send to the production manifold. Moreover, the dilution water is mixed with the oil at the output of the second stage of separation (after second pressure valve and in pressure of about $440 \mathrm{kPa}$ ) to remove the salt concentration (Figure 4.3). In addition, as temperature affects water droplet settling by its effect on oil viscosity (ABDEL-AAL et al., 2015), the dilution water is heated (about $60^{\circ} \mathrm{C}$ ) before it mixes with the processed oil. As shown in Figure 4.3, the hot fluid of dilution water heat exchanger (water heater) also supplied by from the WHRU.

Figure 4.3 - Simplified scheme of three-stage separation train.

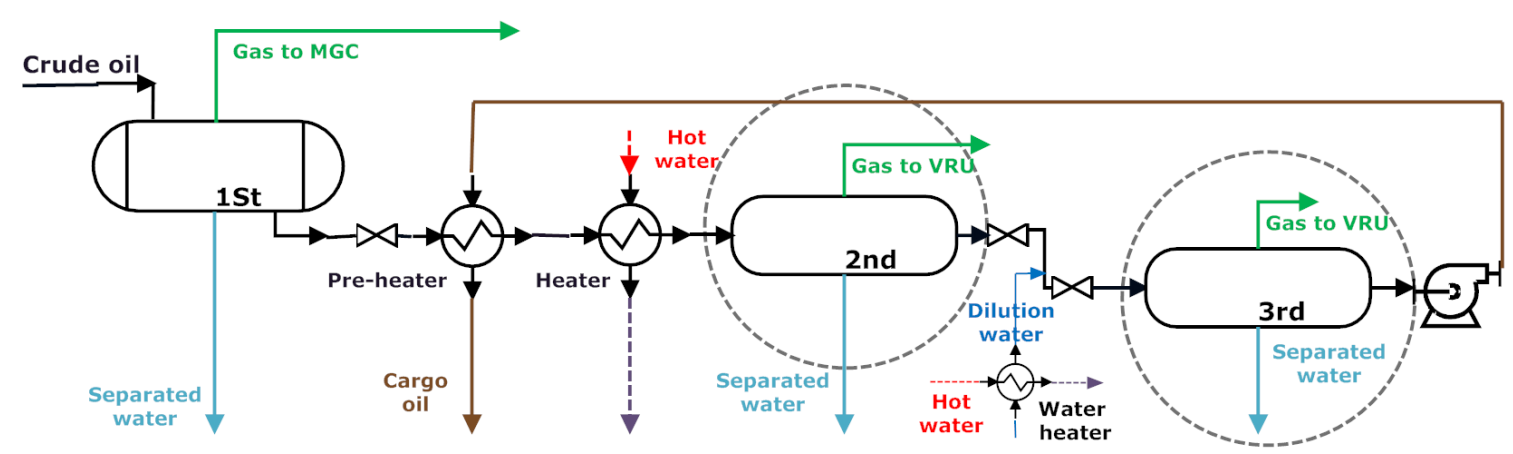

Source: Author 
Figure 4.4 - Simplified scheme of degasser and electrostatic treatment.

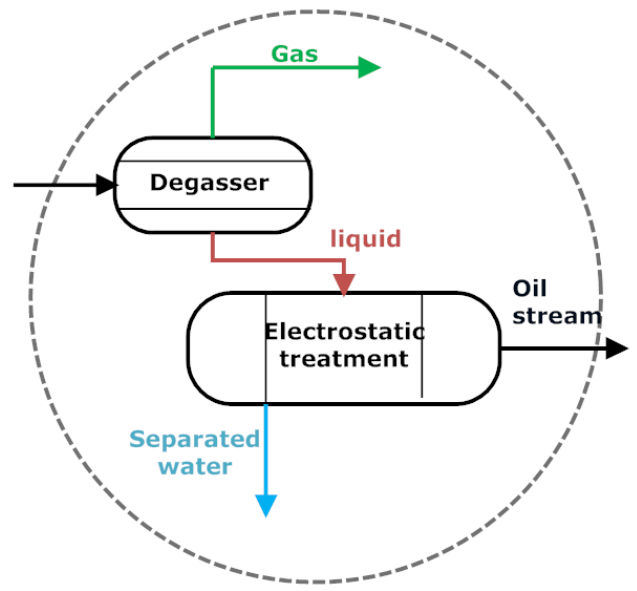

Source: Author

The output stream of the second stage of the separation train loses its pressure again in third pressure valve and then enters in the third stage. The second pair of the separator tank (Figure 4.4) in the third stage operates in the same way. However, the pressure level is lower (about $240 \mathrm{kPa}$ ) in order to provide an incremental separation before transmitting oil to the cargo tanks. The pressure at the third stage of the separator can be reduced to atmospheric pressure (around $100 \mathrm{kPa}$ ) so that the heavy gas components can boil out. In some processes in which the initial temperature is low, it may be necessary to heat the liquid again before flashing to achieve high separation performance of the heavy components (DEVOLD, 2006). The separated gas is sent to the first stage of the VRU, then to the second stage of VRU and finally, is directed to the MGC (Figure 4.5). The separated water in this stage is oriented to discharge.

\subsubsection{Gas Treatment Units}

Gas processing includes treatment and compression processes to meet operational and delivery specifications. Gas treatment units are divided into five systems: Vapor recovery unit (VRU), Main gas compression (MGC), Gas dehydration system (GDS), Hydrocarbon dew point control system (HDP) and Exportation gas compression (EGC).

\subsubsection{Vapor Recovery Unit (VRU)}

Regarding gas treatment units, the separated gas in the first separation stage (high-pressure gas) is sent directly to the MGC, as depicted before in Figure 4.3. As shown in Figure 4.5, the separated gas from the second stage (medium-pressure gas) is compressed in the second stage of VRU and similarly, the third separation stage gas (low-pressure gas) goes to the first stage of VRU to compress at the initial feed pressure of MGC. There are a cooler heat 
exchangers and scrubber in each stage of VRU and separated liquid streams are routed in ST as illustrated in Figure 4.5. Scrubbers are two-phase separators that used to recover liquids condensed in coolers. Gas from the third stage of the ST enters the VRU at about $85^{\circ} \mathrm{C}$ and $236 \mathrm{kPa}$, and the gas from the second stage is recovered at approximately 90 ${ }^{\circ} \mathrm{C}$ and $770 \mathrm{kPa}$. The temperature for the cooling stages is $40^{\circ} \mathrm{C}$. The final compression pressure is $1550 \mathrm{kPa}$ and the gas is cooled at $40{ }^{\circ} \mathrm{C}$ and directed to the MGC.

Figure 4.5 - Simplified scheme of Vapor Recovery Unit.

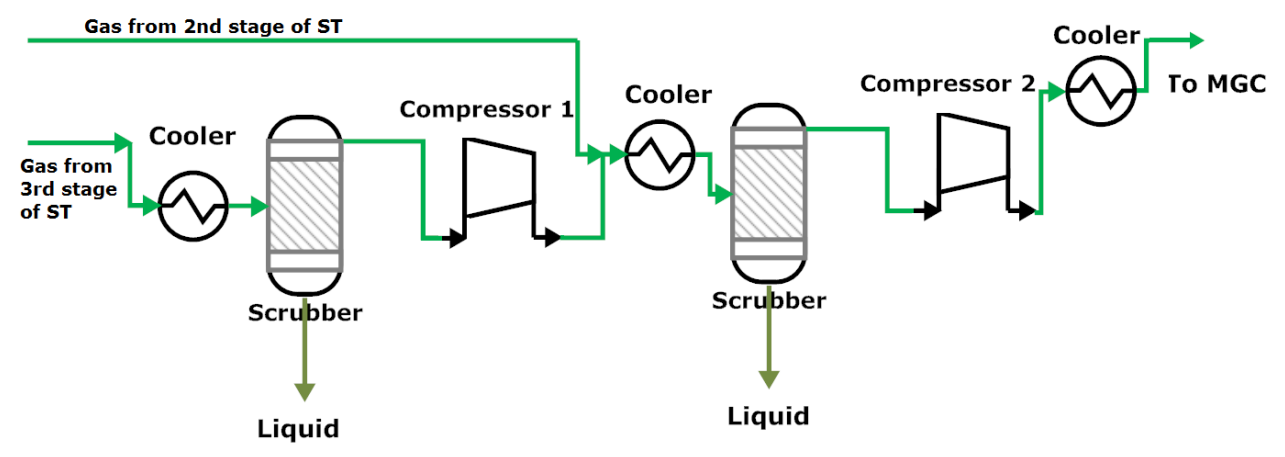

Source: Author

\subsubsection{Main Gas Compression (MGC)}

A simplified scheme of the MGC is shown in Figure 4.6. The treated and compressed gas from VRU's stages is passed from the scrubbers to remove any liquid content. The discharge pressure of these compressors (three compressors) depends on operating mode and it is varying from 7018 to $8500 \mathrm{kPa}$, and then, the compressed gas is routed to the coolers in a temperature of $40{ }^{\circ} \mathrm{C}$. At the last step, there is a separator to remove the liquid fraction (Figure 4.6).

Figure 4.6 - Simplified scheme of Main Gas Compression unit.

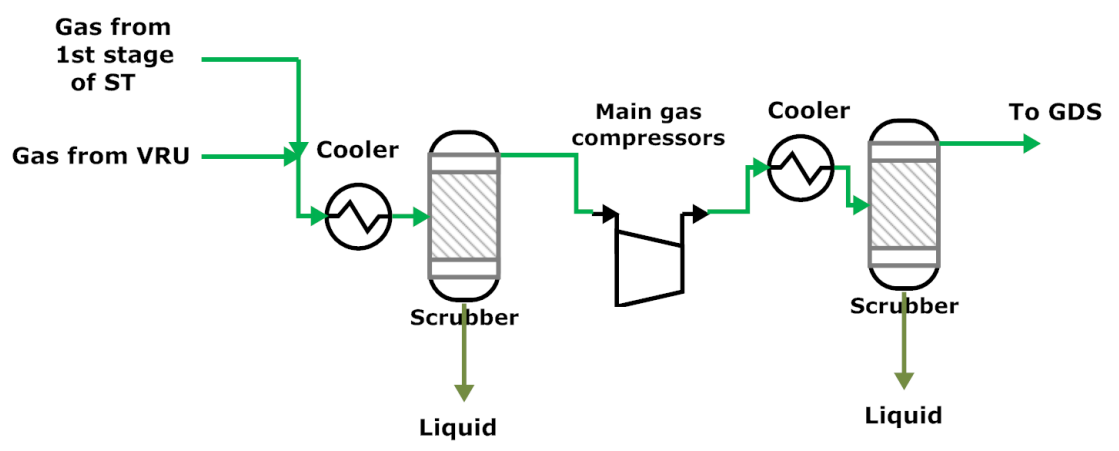

Source: Author 


\subsubsection{Gas dehydration System (GDS)}

The Gas Dehydration system is part of the Gas Treatment units required to meet the specifications in the produced natural gas from the production wells prior to be used as fuel gas, gas-lift, exportation gas and injection gas. The gas stream from MGC at $40^{\circ} \mathrm{C}$, is cooled down in the gas cooler until $26^{\circ} \mathrm{C}$, resulting in a mixed phase flow at about $26^{\circ}$. This mixed phase stream is the feed of the GDS. The pressure of this mixed phase stream may vary from 6500 up to $8100 \mathrm{kPa}$. As Figure 4.7 shows, the dehydrated gas stream from GDS shall comply with water content less than 1 ppmv and will be routed to HDP. The GDS consists of 3 (three) vessels containing the desiccant bed for water (molecular sieves). Upstream the vessels, there is a gas coalescer filter in order to remove condensate. However, in present simulation, this dehydration processes by desiccant bed for water were not modeled in detail and a splitter unit was used instead. A simplified scheme of the GDS is presented in Figure 4.7.

The modeling of molecular sieves was simulated and simplified regarding technical reports and its efficiency. Thus, a splitter unit was used to perform the adapted output compositions and conditions.

Figure 4.7 - Simplified scheme of Gas Dehydration System.

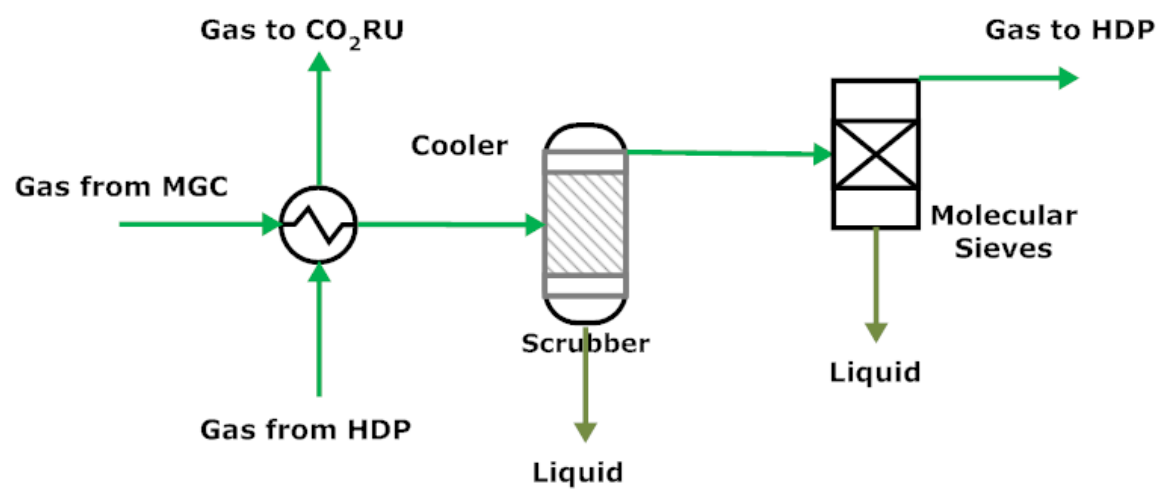

Source: Author

\subsubsection{Hydrocarbons Dew Point Control System (HDP)}

The Hydrocarbon Dew Point Control System is required to achieve a dew point of $10^{\circ} \mathrm{C}$ and $5350 \mathrm{kPa}$ and consists of the following equipment as shown in Figure 4.8:

- Gas/Liquid exchanger;

- Cold separator and;

- Coalescer filter.

The gas stream from GDS is directed to Gas/Liquid exchanger, where it is cooled down to $21^{\circ} \mathrm{C}$. This stream is directed to the Cold separator passing through the Joule-Thomson 
valve (V1), which drops the gas pressure from about 7517 to $5350 \mathrm{kPa}$ and, consequently, drops the temperature down to $10^{\circ} \mathrm{C}$. The gas stream enters the Cold separator where the condensate is separated in order to guarantee the dew point specification at $5350 \mathrm{kPa}$ and $10^{\circ} \mathrm{C}$. As shown in Figure 4.8, the condensate stream flows from the bottom of the separator and through Valve V3, which drops the condensate pressure from 5350 to 870 $\mathrm{kPa}$ and, consequently, drops the stream temperature down to $-25^{\circ} \mathrm{C}$. This stream is directed to Gas/Liquid exchanger to cool down the gas inlet stream and goes to VRU.

The temperature in the Cold Separator is controlled by TC installed in the gas stream outlet, which regulates $V_{1}$ pressure drop. In case of high pressure in the gas inlet stream (Pc), the gas outlet stream temperature control will be switched to LC, which will actuate in V2, directing part of the gas outlet stream to the condensate stream in order to guarantee the dew point temperature in case of insufficient condensate as displayed in Figure 4.8 .

Figure 4.8 - Simplified scheme of Hydrocarbons Dew Point Control System.

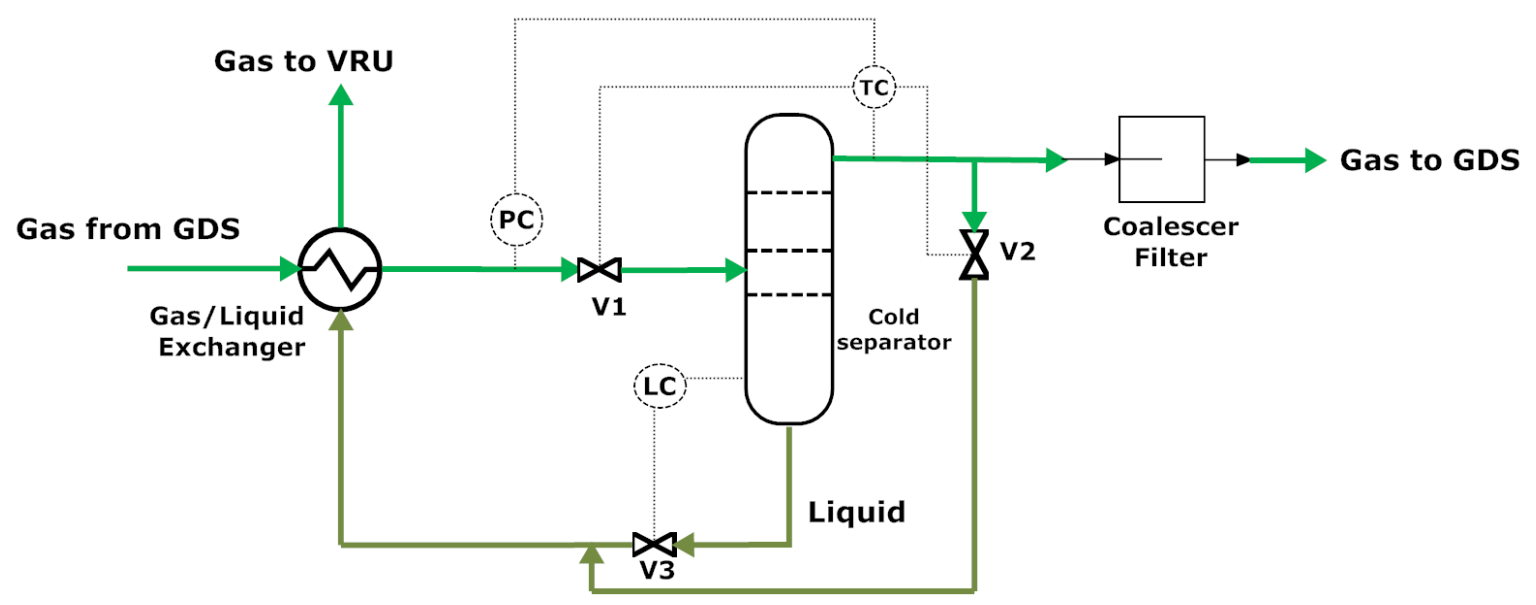

Source: Author

\subsubsection{Exportation Gas Compression System (EGC)}

In some operation modes (will be detailed in subsection 4.1.5), treated gas stream from $\mathrm{CO}_{2} \mathrm{RU}$ is directed to $\mathrm{EGC}$ and, then, to Gas-Lift or IGC. Thus, the gas from $\mathrm{CO}_{2} \mathrm{RU}$ is totally or partially by-passed and the untreated gas stream is also directed to EGC and, then, to IGC. This system is composed of a two-stage compression unit, a 1 st and 2 nd stages scrubbers and a 1st and 2nd stages coolers. The Gas stream enters the EGC at around $5322 \mathrm{kPa}$ (max.) and $38^{\circ} \mathrm{C}$. The gas stream is directed to EGC 1st stage suction scrubber that promotes the separation of condensate in order to guarantee that no liquid will be sent to the gas compressor. This gas stream is then routed to EGC 1st stage. This unit consists of a two-stage compressor, an oil separation and circulation system. The 1st stage discharge pressure will be around $10,981 \mathrm{kPa}$ and shall be confirmed by the supplier. 
As shown in Figure 4.9, the 1st stage compressed gas outlet stream is directed to EGC 1st stage cooler to be cooled down to $40^{\circ} \mathrm{C}$ (max) and then to 2nd stage scrubber. EGC 2 nd stage suction scrubber operates at $11,480 \mathrm{KPa}$ and promotes the separation of condensate. The gas stream is routed to EGC 2nd stage, where it is compressed to a pressure of 25,070 $\mathrm{kPa}$ and, then, to EGC 2nd stage cooler to be cooled down to $40^{\circ} \mathrm{C}$ ( $\max$ ). The gas flow is directed to gas exportation / gas-lift or gas injection (Figure 4.9).

Figure 4.9 - Simplified scheme of Exportation Gas Compression unit.

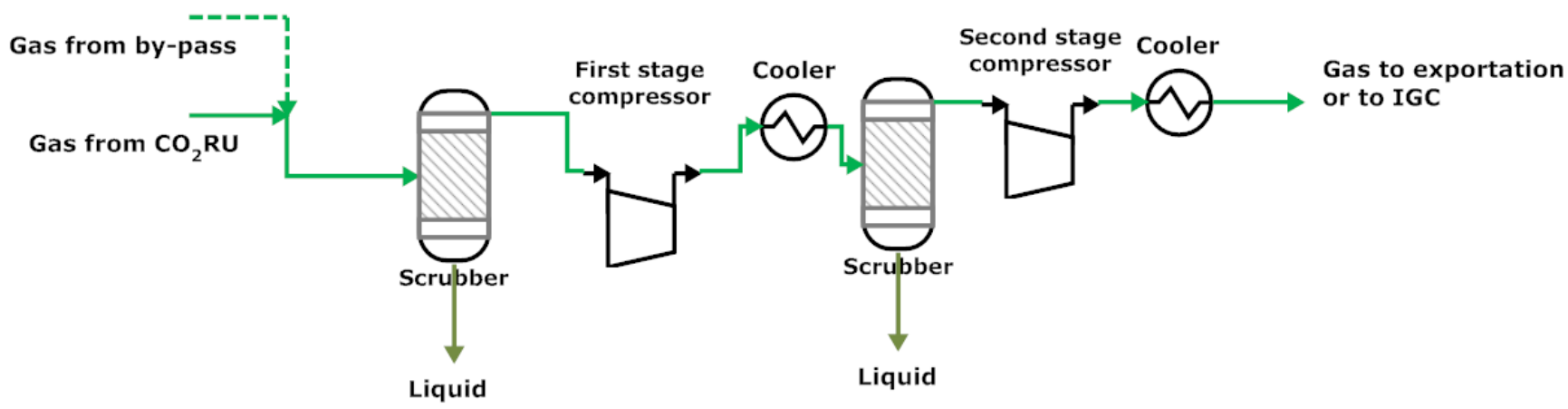

Source: Author

\subsection{3 $\mathrm{CO}_{2}$ Treatment Units}

$\mathrm{CO}_{2}$ treatment units required to meet the specifications in the produced natural gas and $\mathrm{CO}_{2}$ preparation for injection. It consists of three units: $\mathrm{CO}_{2}$ removal unit (CO2RU), $\mathrm{CO}_{2}$ compression $\left(\mathrm{CO}_{2} \mathrm{C}\right)$ and Injection gas compression (IGC).

\subsubsection{1 $\mathrm{CO}_{2}$ Removal Unit $\left(\mathrm{CO}_{2} \mathrm{RU}\right)$}

The $\mathrm{CO}_{2}$ Removal Unit is part of the $\mathrm{CO}_{2}$ Treatment. Since the $\mathrm{CO}_{2}$ content of the gas treatment inlet may vary from $3 \%$ to $60 \%$, a $\mathrm{CO}_{2} \mathrm{RU}$, based on membrane technology, was selected to achieve the required specification of $3 \%$. The $\mathrm{CO}_{2} \mathrm{RU}$ will handle $6.0 \mathrm{MMm}^{3} / \mathrm{d}$ (max.) of produced natural gas and it will be installed downstream the gas pretreatment systems to provide the gas inlet conditions required for this unit, as following (Figure 4.10):

- Dehydration Unit: to achieve 1 ppmv water content at outlet stream;

- Hydrocarbon Dew Point Control Unit: to achieve a dewpoint of $10^{\circ} \mathrm{C}$ and $5350 \mathrm{kPa}$.

The gas stream enters the Unit at 5,300 $\mathrm{kPa}$ and $30-35^{\circ} \mathrm{C}$ and is directed to a pre-heater and then the superheat gas is routed to pre-membrane section. At this stage, $\mathrm{CO}_{2}$ is removed and the residual gas produced, feeds the membrane intermediate heater to guarantee the level of gas superheat primary to be routed to second membrane section. 
The residual stream (hydrocarbon rich gas) from $\mathrm{CO}_{2} \mathrm{RU}$ shall comply with the $\mathrm{CO}_{2}$ content less than $3 \%$ molar and will be routed to exportation, re-injection. The permeate stream $\left(\mathrm{CO}_{2}\right.$ rich gas) from $\mathrm{CO}_{2} \mathrm{RU}$ will be routed to compression aiming the $\mathrm{CO}_{2}$ re-injection and oil recovery improvement. As the lack of efficiency data and the membrane details, the $\mathrm{CO}_{2}$ membrane is considered as a black box using a splitter in the simulation.

Figure 4.10 - Simplified scheme of $\mathrm{CO}_{2}$ Removal Unit.

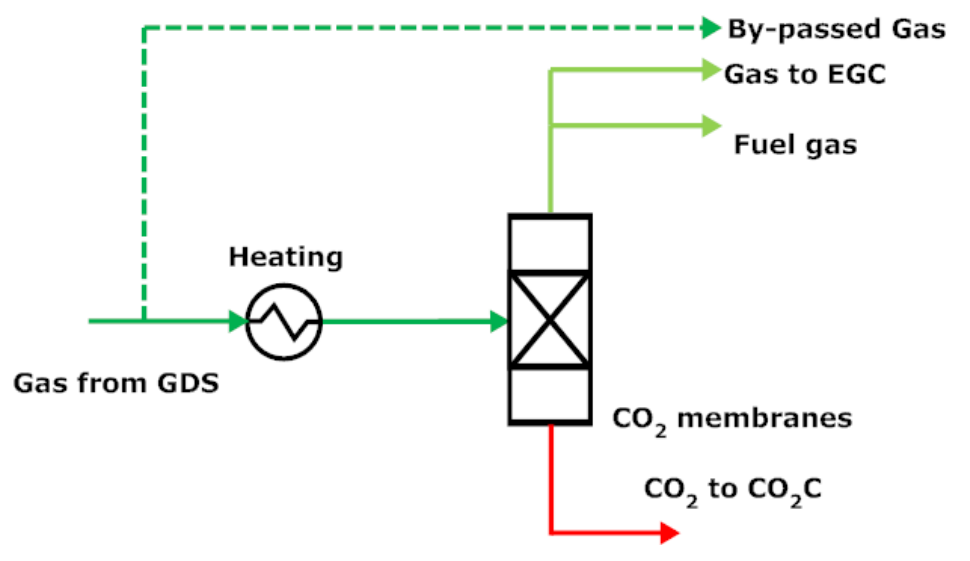

Source: Author

\subsubsection{2 $\mathrm{CO}_{2}$ Compression system $\left(\mathrm{CO}_{2} \mathrm{C}\right)$}

The permeate gas stream from $\mathrm{CO}_{2} \mathrm{RU}$ is directed to $\mathrm{CO}_{2} \mathrm{C}$ System. This system is composed of a four-stage turbo-compression unit, a 1st, 2nd, 3rd and 4th stage suction scrubbers and a 1st, 2nd, 3rd and 4th stage discharge coolers as can be seen in Figure 4.11.

The permeate stream enters the $\mathrm{CO}_{2} \mathrm{C}$ at $400 \mathrm{kPa}$ and $40^{\circ} \mathrm{C}$. This $\mathrm{CO}_{2}$ rich gas stream is directed to $\mathrm{CO}_{2}$ Compressor 1st stage. Each stage gas inlet stream is first directed to the $\mathrm{CO}_{2}$ Compression 1st / 2nd / 3rd / 4th stages suction scrubbers. One for each stage, which promotes the separation of condensate, in order to guarantee that no liquid will be sent to the compressor. The $\mathrm{CO}_{2} \mathrm{C}$ 1st stage suction pressure is $400 \mathrm{kPa}$ and 4 th stage discharge pressure will be $25,110 \mathrm{kPa}$.

Each stage compressed gas outlet stream is directed to $\mathrm{CO}_{2} \mathrm{C} 1 \mathrm{st} / 2 \mathrm{nd} / 3 \mathrm{rd} / 4$ th stages discharge coolers, to be cooled down to $40^{\circ} \mathrm{C}(\max )$. The gas flow in each $\mathrm{CO}_{2} \mathrm{C}$ Unit is directed to gas injection compression (Figure 4.11).

Note that the required injection pressure is adjusting regarding the percentage of $\mathrm{CO}_{2}$ content in the gas stream and gas flow rate as shown in Table B.1. 
Figure 4.11 - Simplified scheme of $\mathrm{CO}_{2}$ Compression Unit.

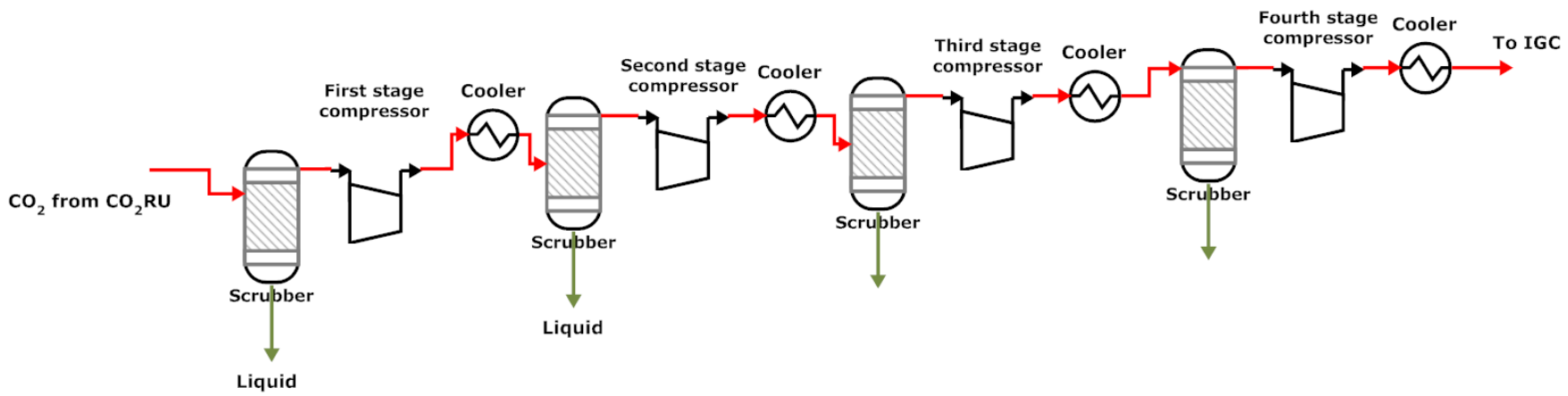

Source: Author

\subsubsection{Injection Gas Compression unit(IGC)}

Injection Gas Compression System receives treated gas from EGC and/or $\mathrm{CO}_{2}$ rich compressed gas from $\mathrm{CO}_{2} \mathrm{C}$, or in case of $\mathrm{CO}_{2} \mathrm{RU}$ total or partially bypass, untreated gas from gas exportation unit, depending on the operation mode. The gas stream is compressed and directed to $\mathrm{CO}_{2}$ injection wells. The gas stream enters the IGC at 25,061 kPa (max.) and $40^{\circ} \mathrm{C}$ depending on gas composition and is directed to IGC suction scrubber that promotes the separation of condensate in order to guarantee that no liquid will be sent to the gas compressor as shown in Figure 4.12.

This gas stream is then directed to Injection Gas Compression Unit that consists of a single stage compressor. The discharge pressure varies between 55,000 and 31,125 kPa in accordance with gas stream composition. The compressed gas outlet stream is directed to IGC Cooler, to be cooled down to $40^{\circ} \mathrm{C}$ (max). The gas flow goes to each compressor gas discharge line and directed to $\mathrm{CO}_{2}$ injection headers (Figure 4.12).

Figure 4.12 - Simplified scheme of Injection Gas Compression Unit.

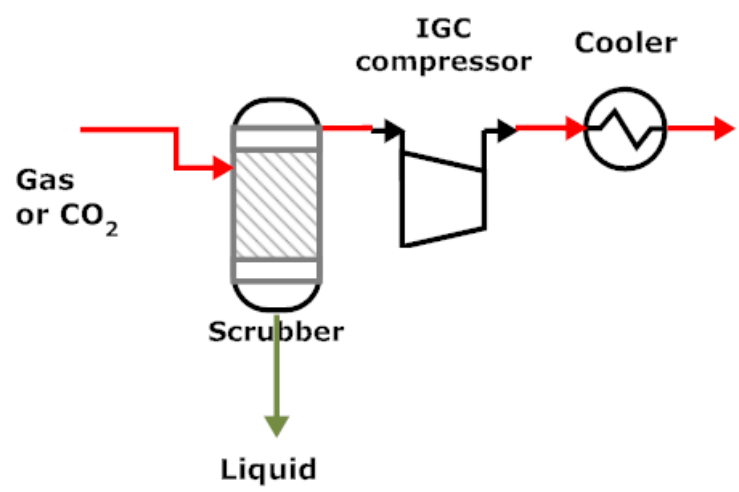

Source: Author 


\subsubsection{Utility plants}

The utilities consist of a power and heat generation unit, which includes one or several gas turbine (GT) units combined with a Waste Heat Recovery Unit (WHRU), and the cooling system, which is a heat exchanger network where seawater is pumped on-site. For heating fluid such as high-pressure liquid, glycol or water is used to transfer heat from one process to another (is not considered in this simulation).

\subsubsection{Power and heat generation unit}

As regards the power and heat generation unit, a real performance data of an aero-derivative gas turbine (RB211G62 DLE $60 \mathrm{~Hz}$ turbine) with offshore application is obtained from GATECYCLE ${ }^{\text {TM }}$ commercial software (General Electric , 2013). The gas turbine produces a power of $27.90 \mathrm{MW}$ with an exhaust temperature of $549^{\circ} \mathrm{C}$ at full load and site temperature. As described in Figure 4.13, the exhaust gas is routed to the WHRU to heat the hot water system. Then, the heated water is sent to heat exchangers of the separation train and other consumers. The hot water and cooling water systems are simulated as an integration heat system in order to provide heating fluid for the heat exchangers and to remove heat for the proposed units, respectively. The feed water supplies the necessary mass flow from seawater.

Figure 4.13 - Simplified scheme of Gas Turbine and WHRU.

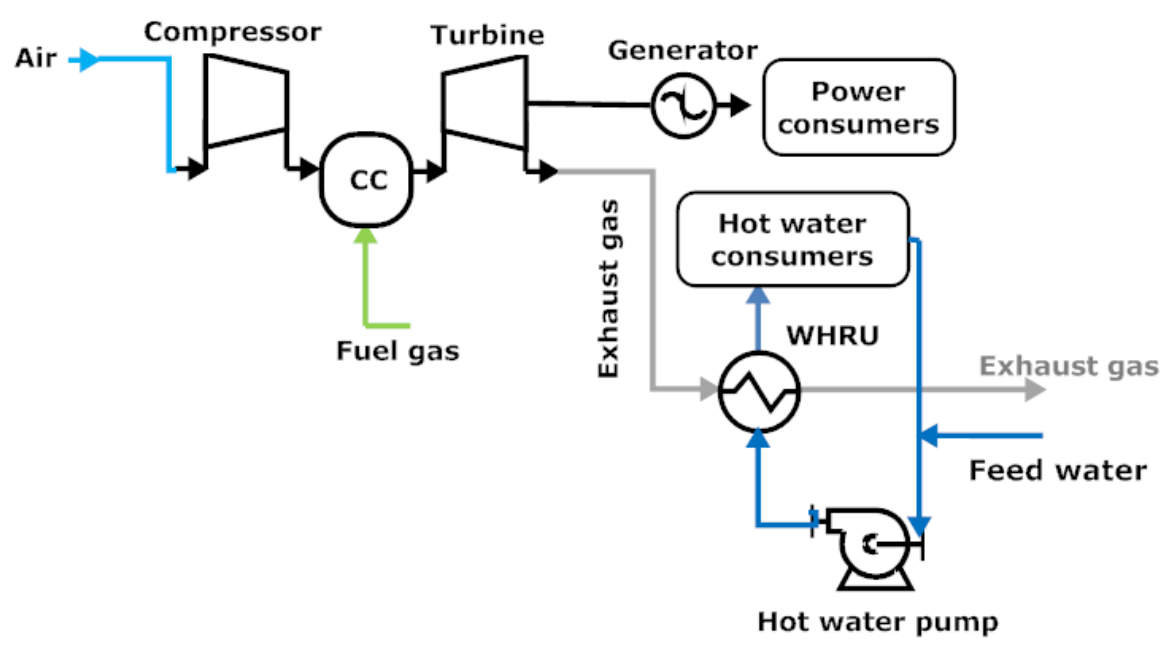

Source: Author

According to site temperature of FPSO and demand of electric power (inputs), GATECYCLE ${ }^{\text {TM }}$ calculates the output temperature of gas exhaust, mass flow rate of gas exhaust and thermal efficiency of the selected gas turbine. Thus, these performance data are extracted from GATECYCLE $^{\text {TM }}$ and as a function of inputs and are inserted in ASPEN HYSYS V9.0. Therefore, regarding the variation of the power demand (and site temperature in specific 
condition), ASPEN HYSYS provides required information of heat water temperature, water mass flow and efficiency of gas turbine. The simulation of aero-derivative gas turbine (RB211G62 DLE 60Hz turbine) in GATECYCLE ${ }^{\mathrm{TM}}$ and using this performance data is explained in Appendix A .

\subsubsection{Hot Water system}

The Hot Water System is a closed system that uses pressurized fresh water. Heat is recovered from the gas exhausted from each gas turbine (WHRU- Waste Heat Recovery Unit). Hot Water at $100^{\circ} \mathrm{C}$, is pumped by the hot water circulation pump, through the Turbo generator Waste Heat Recovery Unit, where it is heated up to $130^{\circ} \mathrm{C}$. Hot Water leaves the consumers at $100^{\circ} \mathrm{C}$ (min.). Make up water is fed to the system by Hot Water Make up pumps from seawater (Figure 4.13).

\subsubsection{Cooling Water system}

The cooling water system consists of a closed fresh water system, indirectly (heat exchanger) cooled by sea water. The seawater at $25^{\circ} \mathrm{C}$ is pumped into the heat exchanger, where cooling water is cooled down from 55 to $35^{\circ} \mathrm{C}$, to the consumers and, then, back to pump suction. The seawater at around $45^{\circ} \mathrm{C}$ is rejected to the sea. In fact, the last destination of free water will be the sea, according to the CONAMA 303/07 legal standard; the maximum concentration of oils and grease that can be disposed in water is $29 \mathrm{mg} / \mathrm{L}$.

In the first stage of the water treatment system, a series of small vessels, called hydrocyclones, is included. The stream accelerated by the continuous decrease in the vessel diameter makes a centrifugal force separate heavier components such as water and solids from the wall and oil rejects on the opposite side of the water output, in the central portion.

Finally, the floater vessel removes the remaining oil parcels agglomerated in water, with the aid of an upward air stream. If the analyzer finds a value above that allowed by law, the flow is redirected from the sea output to specific tanks or it will be injected into a reservoir through injection wells.

\subsubsection{Water Injection System}

Treated sea water is pumped by booster injection water pumps through injection water heat exchanger to main injection water pump and, then, to be injected in WAG (water alternating gas) injection wells for EOR at a pressure of $25000 \mathrm{kPa}$ and $44^{\circ} \mathrm{C}$. 


\subsubsection{Operation Scenarios}

Figure 4.14, 4.15 and 4.16 show the simplified schemes of the FPSO processes for operation modes 1, 2 and 3, respectively. Mode 1 corresponds to the maximum amount of oil/gas in crude oil; Mode 2 refers to basic sediment and water (BS\&W), comprising $50 \%$ of the stream and; Mode 3 indicates the maximum quantity of water/ $\mathrm{CO}_{2}$. Mode 1 represents the typical early life condition and Mode 3 the end of life condition of an oil field. As can be seen in Figure 4.14, 4.15 and 4.16, a part of the gas produced offshore is usually used as a fuel for the power generation and waste heat recovery unit in gas turbines.

For the operation mode 1, all dehydrated gas passes from $\mathrm{CO}_{2} \mathrm{RU}$ and then is directed to exportation and the separated gas is compressed in the exportation gas compressors. The separated $\mathrm{CO}_{2}$ is compressed by a section of the injection unit as shown in Figure 4.14. For the operation mode 2, a fraction of the dehydrated gas is sent to the $\mathrm{CO}_{2}$ membrane, while the remaining gas is processed directly through a section of the gas exportation unit, and then directed to a section of the $\mathrm{CO}_{2}$ compressors for further compression and injection in the wells (Figure 4.15). The treated gas in the $\mathrm{CO}_{2}$ membrane is mainly used for exportation purposes. Finally, as illustrated in Figure 4.16, in the operation mode with maximum water $/ \mathrm{CO}_{2}$ content (Mode 3 ), all the gas is proposed to be injected into the wells in order to EOR, except the amount required by the gas turbine. After the gas dehydration unit, gas is bypassed the membranes of the $\mathrm{CO}_{2}$ removal unit and is directly sent to the two gas injection steps to reach the required pressure for injection purposes.

Figure 4.14 - The general scheme of oil and gas processing in FPSO - operational mode 1.

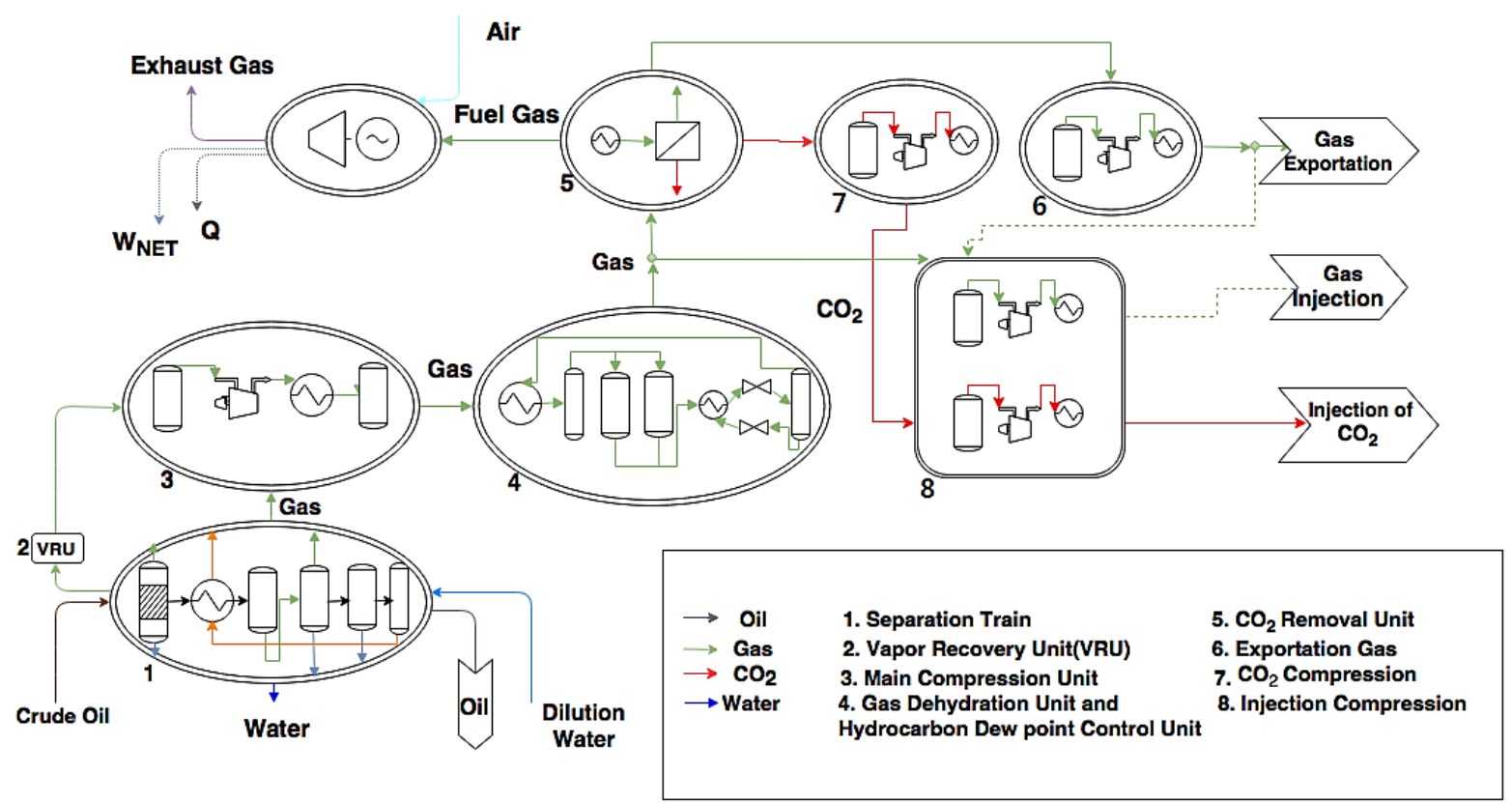


The pressure and temperature condition of each operation mode regarding the used crude oil compositions are different to each other. Because, as explained before, the stabilization and separation of hydrocarbon components vary during each operation mode.

Figure 4.15 - The general scheme of oil and gas processing in FPSO - operational mode 2.

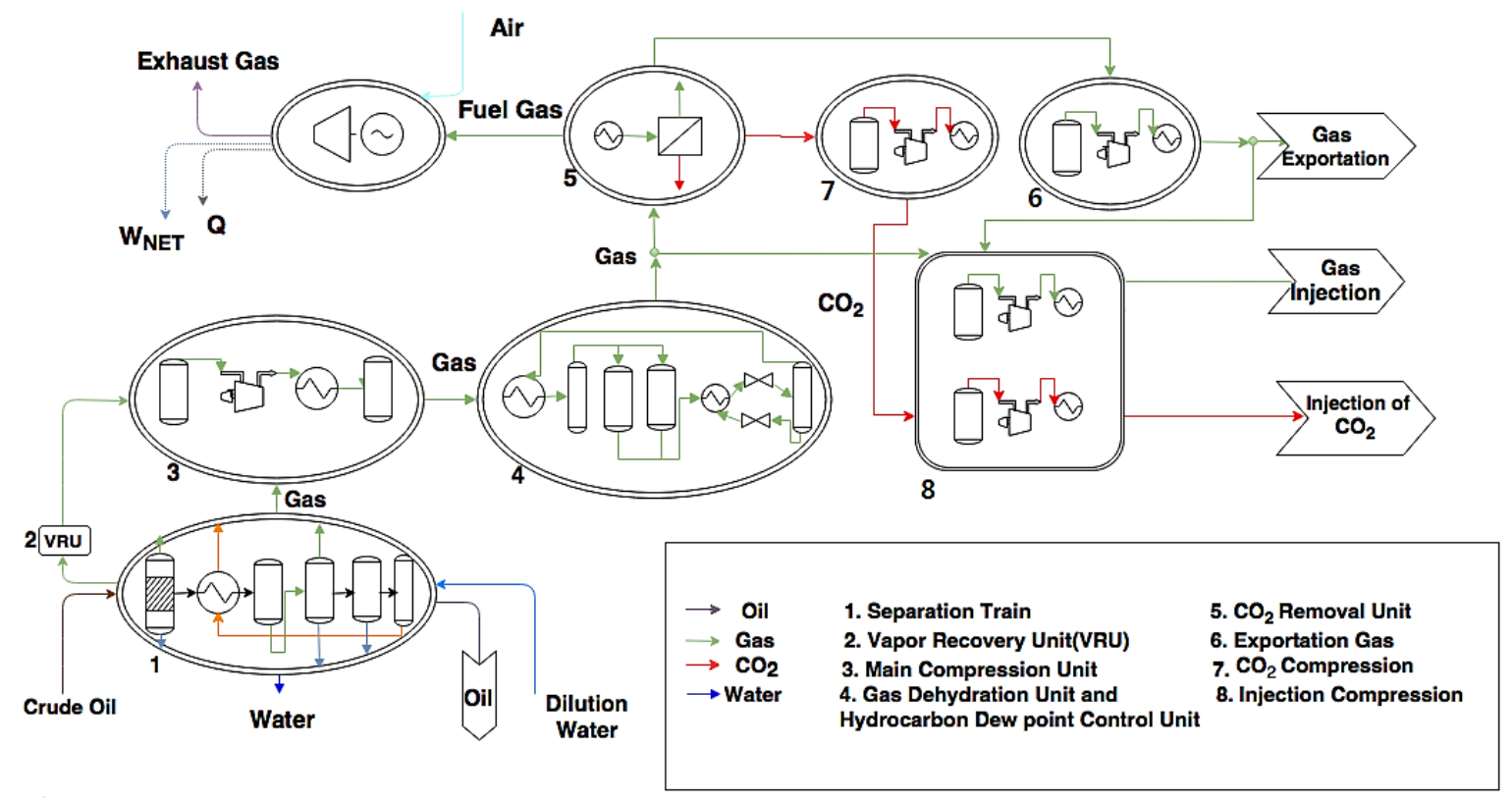

Source: Author

Figure 4.16 - The general scheme of oil and gas processing in FPSO - operational mode 3.

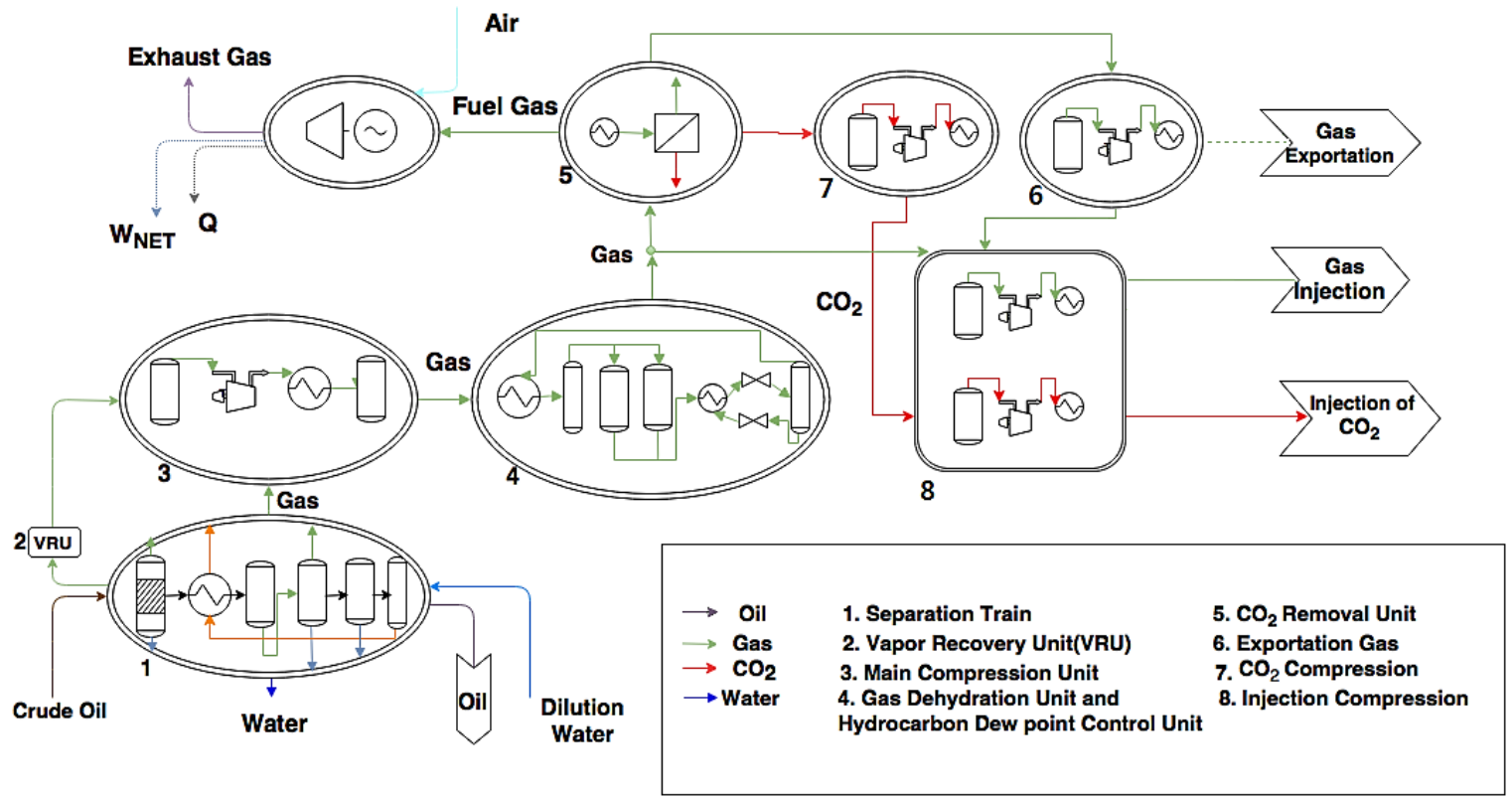

Source: Author 


\subsection{Assumptions for modeling and simulation}

The proposed FPSO is modeled and simulated by using the software ASPEN HYSYS ${ }^{\circledR}$ V9.0 (ASPENTECHNOLOGYINC., 2016), which is based on the Peng \& Robinson Equation of State (PR-EoS)(PENG; ROBINSON, 1976). As shown before, it is most commonly used EoS in offshore simulation works. The convergence of its numerical calculation in the phase envelope was checked by provided data from the current thesis. In the present research, the simulation are performed for the crude oil composition displayed in Table 4.1 and based on the following assumptions:

Table 4.1 - Crude oil composition of the three operational modes (molar fraction).

\begin{tabular}{cccc}
\hline Components & Mode 3 (Max water/CO $\left.\mathrm{CO}_{2}\right)$ & Mode 2 $(50 \%$ BSW) & Mode 1 (Max oil/gas) \\
\hline $\mathrm{H}_{2} \mathrm{O}$ & 0.89774 & 0.83360 & 0 \\
$\mathrm{~N}_{2}$ & 0.00023 & 0.00083 & 0.00490 \\
$\mathrm{CO}_{2}$ & 0.05438 & 0.03009 & 0.16000 \\
$\mathrm{C}_{1}-\mathrm{C}_{4}{ }^{*}$ & 0.03550 & 0.08990 & 0.63820 \\
$\mathrm{C}_{5}{ }^{*}-\mathrm{C}_{12}$ & 0.00569 & 0.02374 & 0.10290 \\
$\mathrm{C}_{13}-\mathrm{C}_{19}$ & 0.00237 & 0.00982 & 0.04670 \\
$\mathrm{C}_{20+}{ }^{* *}$ & 0.00409 & 0.01202 & 0.04730 \\
& & &
\end{tabular}

${ }^{*} \mathrm{C}_{4}$ and $\mathrm{C}_{5}$ includes the $\mathrm{nC}_{4}$ and $\mathrm{iC}_{4}$, and the $\mathrm{nC}_{5}$ and $\mathrm{iC}_{5}$ hydrocarbons, respectively.

** $\mathrm{MW} \mathrm{C}_{20+}$ is 581 and $\mathrm{SG} \mathrm{C}_{20+}$ is 0.9587 .

- The operating pressure and temperature, and the number of stages of the separation train are simulated according to a typical FPSO condition and according to the category of the crude oil composition for separation processes with $\mathrm{GOR}, \mathrm{CO}_{2}$, water and salt content (ABDEL-AAL et al., 2015; GALLO et al., 2017);

- Crude oil production of 150,000 barrels per day, which $4.0 \%, 2.0 \%$ and $0.8 \%$ of the crude oil mass flow rate were considered for dilution water in mode 1, 2 and 3, respectively;

- Temperature and pressure references are $298.15 \mathrm{~K}$ and $101.3 \mathrm{kPa}$, respectively;

- For the separation efficiencies of the dehydration and $\mathrm{CO}_{2}$ separation systems, Gas Dehydration Unit is projected to remove $99 \%$ of the water contained in the gas stream and maximum $3 \% \mathrm{CO}_{2}$ content in gas for the $\mathrm{CO}_{2}$ Removal Unit;

- Adiabatic efficiency of $75 \%$ is assumed for all centrifugal compressors;

- Separators, pumps, mixers, splitters and gas turbine are considered adiabatic;

- The pressure for the exported gas is $25 \mathrm{MPa}$ and for injection, $55 \mathrm{MPa}$. 


\subsection{Modeling, Simulation and optimization description}

In this section, the procedure of modeling, simulation and optimization with their details are explained and discussed as Strategy title:

\subsubsection{Strategy}

The structure of this methodology for optimization consists of:

- System modeling using flow sheeting tools as much as possible using real operating and performance data;

- Applying evaluation methods for system analysis;

- Applying a robust statistical method of screening analysis (SS-ANOVA) to identify the most important contributors to values of outputs and;

- System optimization based on powerful evolutionary algorithms and heuristics search such as GA (and/or a hybrid method of GA in order to improve the accuracy).

The fundamental programming base on VBscript is provided during simulation and modeling to couple the integration softwares such as GATECYCLE in order to use real performance data of power and heat generation unit and ESTECO modeFRONTIER as a statistical analyzer and multidisciplinary optimizer of design process software.

Thus, as an initial step, after simulating the proposed system via Aspen HYSYS ${ }^{\circledR}$ and Aspen Simulation Workbook (Aspen Technology, Inc, 2014) (step1), the major energy and power consumers, and details of the desired production are analyzed (step 2) as shown in Figure 4.17 .

Then, the important operating parameters from the literature and evaluation methods are extracted from modeling and the data of model simulation are categorized and transferred through VBScript to be used by a preliminary screening analyzer software (via the Smoothing Splice ANOVA method) to reduce the number of input variables for the optimization procedure (step 3).

As described in Figure 4.17, after that, only the most relevant input parameters are delivered to the optimization procedure and Hybrid GA method (via ModeFRONTIER ${ }^{\mathrm{TM}}$ (Esteco SPA, 2017)) was performed by modifying the value assigned to the input variables as decision variables and analyzing the outputs as defined objectives. Finally, data such as temperatures, pressures, oil production and etc, are extracted from the process models (post-processing) and sent to the next computing step to justify the obtained results (step 4). 
Figure 4.17 - Conceptual structure of the general methodology and computational steps to perform an optimization procedure for proposed FPSO.

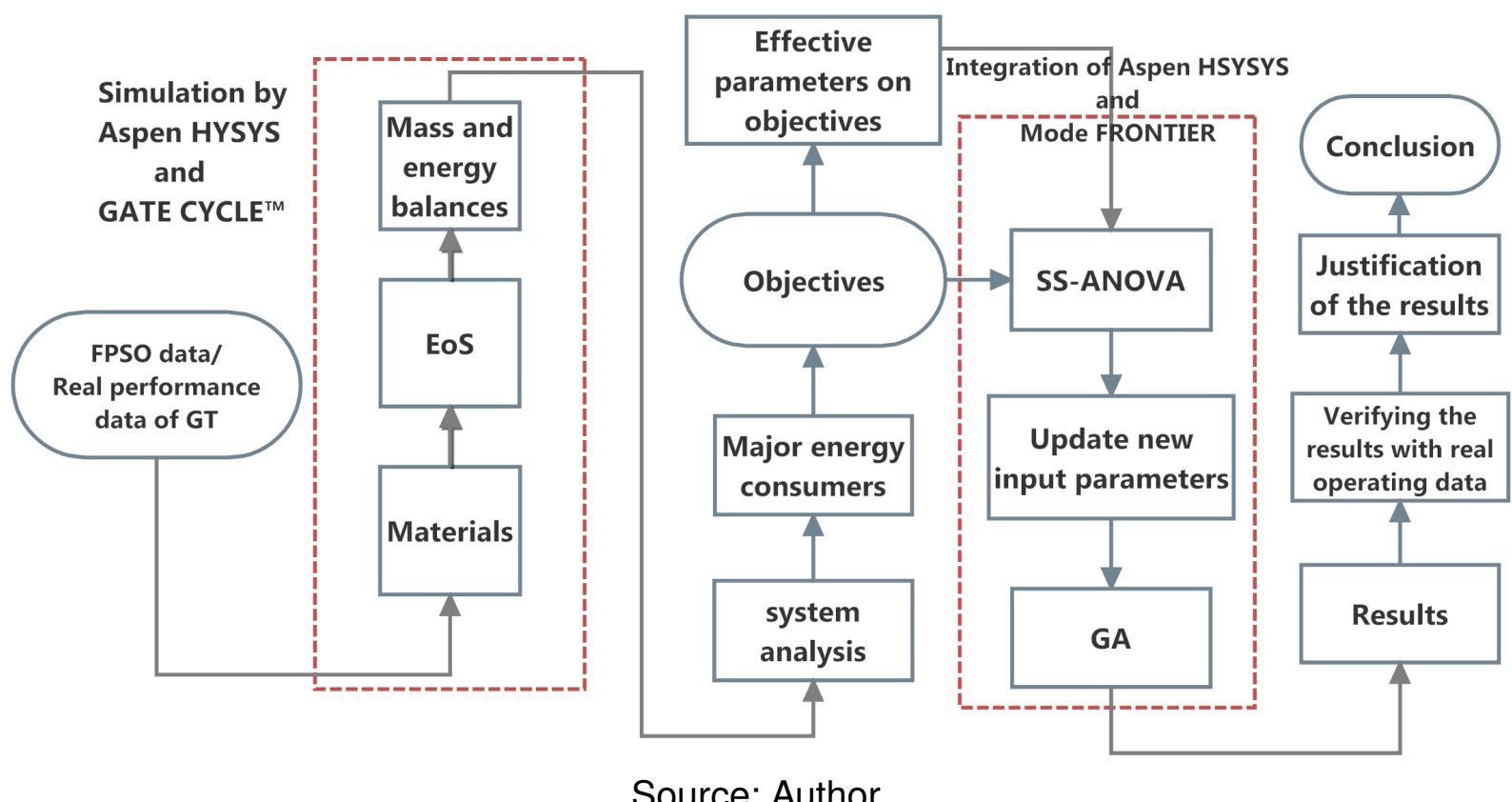

\subsubsection{Simulation of FPSO in HYSYS}

The mass and energy balances for the process of interest are performed using the Aspen HYSYS ${ }^{\circledR}$ software. It is a powerful software used both in the academic and industrial contexts for modeling systems involving complex chemistry, such as carbon dioxide capture, and for predicting the energy demand of chemical processes.

The simulation of the system under study (appendix) is conducted according to the description in sections 4.1 and 4.2 and the details are presented in Appendix $B$ and Figure B.2.

As mentioned, thermodynamic models are used to represent the phase equilibrium behavior and energy level of pure compound and mixture systems by Aspen Hysys (Figure 4.18). 
Figure 4.18 - How Aspen HYSYS Works

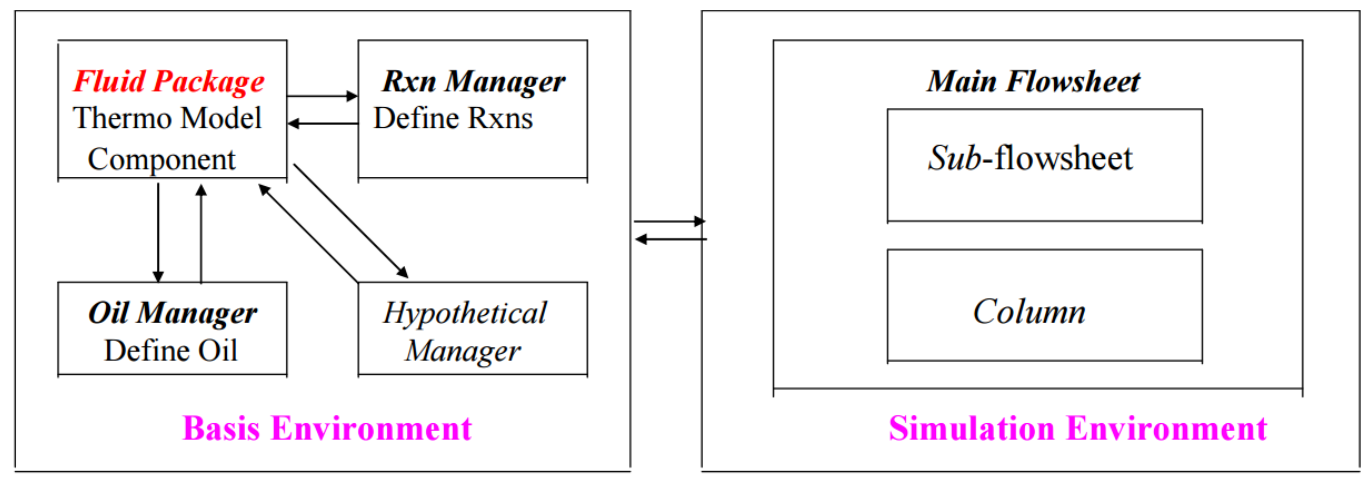

Source: (GUERRA, 2006)

Solving equations of state (EOS) allows calculating the specific volume of a pure component or a mixture of chemicals at a specified temperature and pressure and designing a chemical plant. By knowing this specific volume, it is possible to determine other thermo- dynamic properties. HYSYS offers the enhanced Peng-Robinson (PR) equations of state. The Peng-Robinson equation of state supports the widest range of operating conditions and the greatest variety of systems to generate all required equilibrium and thermodynamic properties directly. The PR package contains enhanced binary interaction parameters for all library hydrocarbon-hydrocarbon pairs (HAMID, 2007).

Peng and Robinson introduced the following EOS (PENG; ROBINSON, 1976):

$$
p=\frac{R T}{V_{m}-b}-\frac{a}{-b^{2}+2 b V_{m}+V_{m}^{2}}
$$

where:

$$
\begin{gathered}
a=\frac{0.457235 R^{2} T_{c}^{2}}{p_{c}} \alpha(T) \\
b=0.07780 \frac{R T_{c}}{p_{c}} \\
\alpha(T)=\left[1+\kappa\left(1-\left(\frac{T}{T_{c}}\right)^{\frac{1}{2}}\right)\right]^{2} \\
\kappa=0.37464+1.54226 \omega-0.26992 \omega^{2}
\end{gathered}
$$

Translated into polynomial form:

$$
\begin{gathered}
A=\frac{a p}{R^{2} T^{2}} \\
B=\frac{b p}{R T} \\
Z^{3}-(1-B) Z^{2}+\left(A-2 B-3 B^{2}\right) Z-\left(A B-B^{2}-B^{3}\right)=0
\end{gathered}
$$


when dealing with multi-component mixtures, mixing rules can be implemented as:

$$
\begin{gathered}
\text { amix }=\sum_{i=1}^{N} \sum_{j=1}^{N} x_{i} x_{j} A_{i j} \\
\text { bmix }=\sum_{i=1}^{N} x_{i} b_{i}
\end{gathered}
$$

where:

$$
A i j=A j i=\left(a_{i} a_{j}\right)^{\frac{1}{2}}\left(1-k_{i j}\right)
$$

The experimental parameters provided by the literature are $T_{c}$ (critical temperatures), $P_{c}$ (critical pressures), $R$ (universal gas constant), $\omega$ (acentric factor), and $k_{i j}$ (binary interaction parameters). The component molar compositions are $x_{i}$ and is $N$ in length.

When an acentric factor $>0.49$ is present, HYSYS uses following corrected form for $\mathrm{k}$ :

$$
k=0.379642+(1.48503-[0.164423+0.016666 \omega] \omega) \omega
$$

For the Peng-Robinson Equation of State, the enthalpy and entropy departure calculations use the following relations:

$$
\begin{gathered}
\frac{H-H^{I D}}{R T}=Z-1-\frac{1}{2^{1.5} b R T}\left[a-T \frac{d a}{d t}\right] \ln \left(\frac{V+\left(2^{2.5}+1\right) b}{V-\left(2^{2.5}-1\right) b}\right) \\
\frac{S-S_{0}{ }^{I D}}{R T}=\ln (Z-B)-\ln \frac{P}{P^{o}}-\frac{A}{2^{1.5} b R T}\left[a-T \frac{d a}{d t}\right] \ln \left(\frac{V+\left(2^{2.5}+1\right) b}{V-\left(2^{2.5}-1\right) b}\right)
\end{gathered}
$$

where:

$\mathrm{H}^{I D}=$ Ideal Gas Enthalpy basis used by HYSYS changes with temperature according to the coefficients on the TDep tab for each individual component. ID indicates Ideal Gas and ${ }^{o}$ indicates reference state,

$$
\begin{gathered}
a=\sum_{i=1}^{n} \sum_{j=1}^{n} x_{i} x_{j}\left(a_{i} a_{j}\right)^{0.5}\left(1-k_{i j}\right) \\
b_{i}=0.077796 \frac{R T_{c}}{P_{c}}
\end{gathered}
$$




$$
\begin{gathered}
a_{i}=0.457235 \frac{\left(R T_{c}\right)^{3}}{P_{c}} \alpha_{i} \\
\sqrt{\alpha_{i}}=1+m\left(1-T_{r}^{0.5}\right) \\
m=0.37464+1.5226 \omega-0.26992 \omega^{2}
\end{gathered}
$$

Energy is transformed from one form to another and transferred between systems, but can neither be created nor destroyed. The energy rate balance at steady state is:

$$
0=\dot{Q}-\dot{W}+\sum_{\text {in }} \dot{m}_{\text {in }}\left(h_{\text {in }}+\frac{1}{2} V_{\text {in }}^{2}+g z_{\text {in }}\right)-\sum_{\text {out }} \dot{m}_{\text {out }}\left(h_{\text {out }}+\frac{1}{2} V_{\text {out }}^{2}+g z_{\text {out }}\right)
$$

where $\dot{Q}$ and $\dot{W}$ account for the net rates of energy transfer by heat and work; $m$ represents the mass flow rate at an inlet or outlet port; $h$ denotes the specific enthalpy of a stream of matter; $V, g$ and $z$ stand for the velocity, the gravitation constant and the height, respectively.

\subsubsection{Sensitivity analysis description}

Screening analysis is often a preliminary step in any optimization procedure that uses a large number of input parameters. The main objective of the screening analysis is to identify the most important contributors to increase/decrease an output value. This preliminary step is performed by a method named Smoothing Spline ANOVA (SS-ANOVA) model, already implemented in the ESTECO modeFRONTIER software. As both pressure and temperature in multistage crude oil separation processes play an important role in the efficiency of thermal systems and separation of hydrocarbons, the input variables are selected from possible operating parameters to range and then are submitted to SS-ANOVA analysis are: output pressure of the first control valve (P1), output pressure of the second stage of the separation train before mixing with dilution water (P2), input pressure of the third stage of separation train (P3), input pressure of dilution water (P4), output pressure of the main gas compressor $(\mathrm{Pc})$, output petroleum temperature in the first heat exchanger (T1), output petroleum temperature in the second heat exchanger (T2) and dilution water temperature (T3).

Furthermore, technical constraints of the whole plant are assessed during the screening analysis, in order to avoid: (i) unfeasible separators performances; (ii) temperature cross in heat exchangers and; (iii) the decrease of the volume of the oil and gas production( exportation). 
The selected pressure parameters influence the separation performance, which can result in exportation oil with varying quantities of dissolved gas that tends to come out of the storage liquid. Another consequence of pressure variations is the quantity of intermediate-heavy hydrocarbons that entrains the gas flows, changing the compressor shaft work. On the other hand, the variation of the selected pressure parameters and temperatures can change the required heat demands of the separation train. Finally, these parameters were chosen after a linear dependency check.

Table 4.2 shows the eight input parameters, the operating ranges and constraints used for the screening analysis and Figure 4.19a and 4.19b show the location of each input parameter in the FPSO plant. As explained in the previous sections, a three-stage separation train has been simulated and modeled for: oil pre-heating, oil heating, degassers and electrostatic treatment. Moreover, the output pressure of MGC's compressor is another considered input parameter.

Table 4.2 - Input parameter ranges and their respective constraints

\begin{tabular}{ccc}
\hline Input parameters & Operational range & Constraints \\
\hline & & \\
$\mathrm{P}_{1}$ & $101.5-2000(k P a)$ & $\mathrm{P} 1>\mathrm{P} 2$ \\
$\mathrm{P}_{2}$ & $101.5-1050(k P a)$ & $\mathrm{P} 2>\mathrm{P} 3$ \\
$\mathrm{P}_{3}$ & $101.5-1050(k P a)$ & - \\
$\mathrm{P}_{4}$ & $101.5-1050(k P a)$ & - \\
$\mathrm{P}_{C}$ & $7018-8500(k P a)$ & - \\
$\mathrm{T}_{1}$ & $30-80\left({ }^{\circ} \mathrm{C}\right)$ & - \\
$\mathrm{T}_{2}$ & $37-110\left({ }^{\circ} \mathrm{C}\right)$ & - \\
$\mathrm{T}_{3}$ & $80-110\left({ }^{\circ} \mathrm{C}\right)$ & - \\
\end{tabular}

The flowchart in Figure 4.20 shows the steps to perform the screening analysis as explained in subsection 4.3.1. Design space, based on the operating range of the selected input parameters and constraints, is first generated by the Uniform Latin Hypercubes (ULH) algorithm and then if it is necessary, it filled by Incremental Space filler (ISF). From an initial distribution, Aspen HYSYS $\AA$ software is the solver and provides the numerical simulations. After the initial sampling points are selected, the maximum collinearity index is checked until this index is close to the unity. If not, new sampling points are added to the design space and the process continues until the collinearity index criteria is achieved. 
Figure 4.19 - Sketch of the proposed FPSO with.

(a) Three-stage separation train

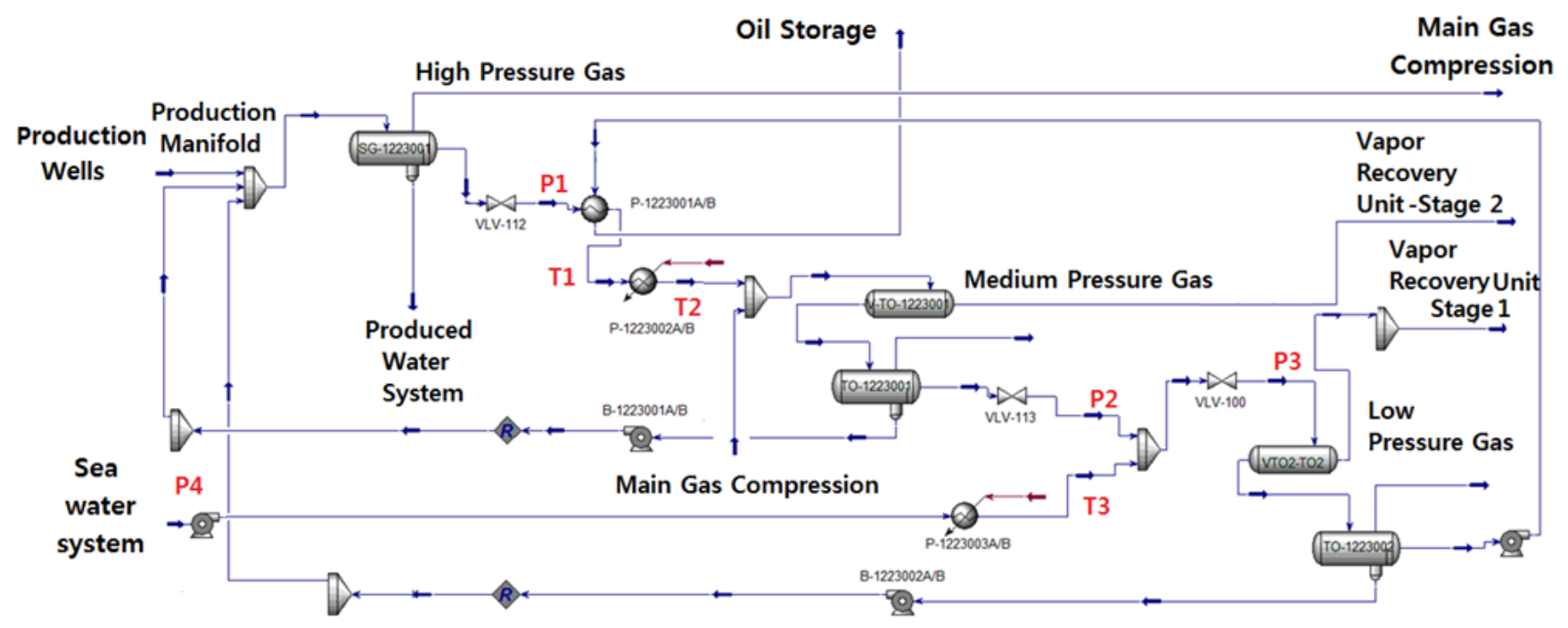

(b) main gass compression unit

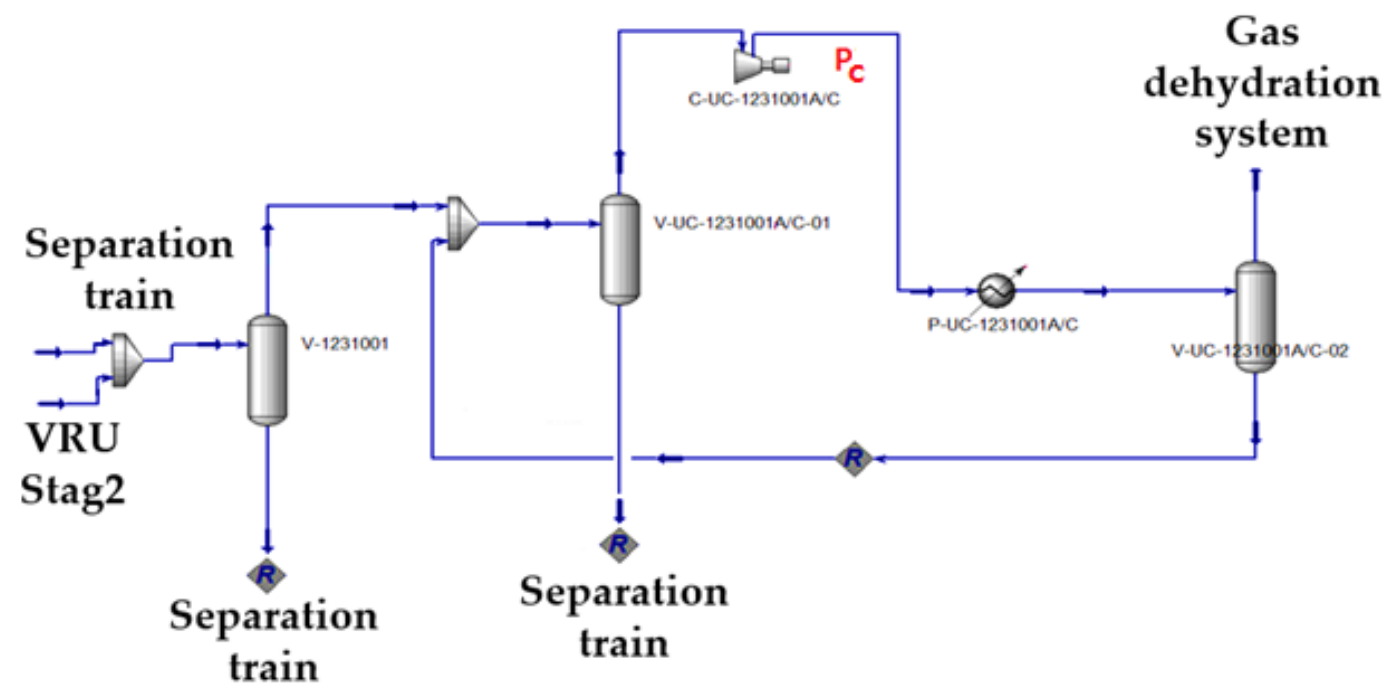

Source : Auhtor 
Figure 4.20 - Flowchart indicating the processes for screening analyses

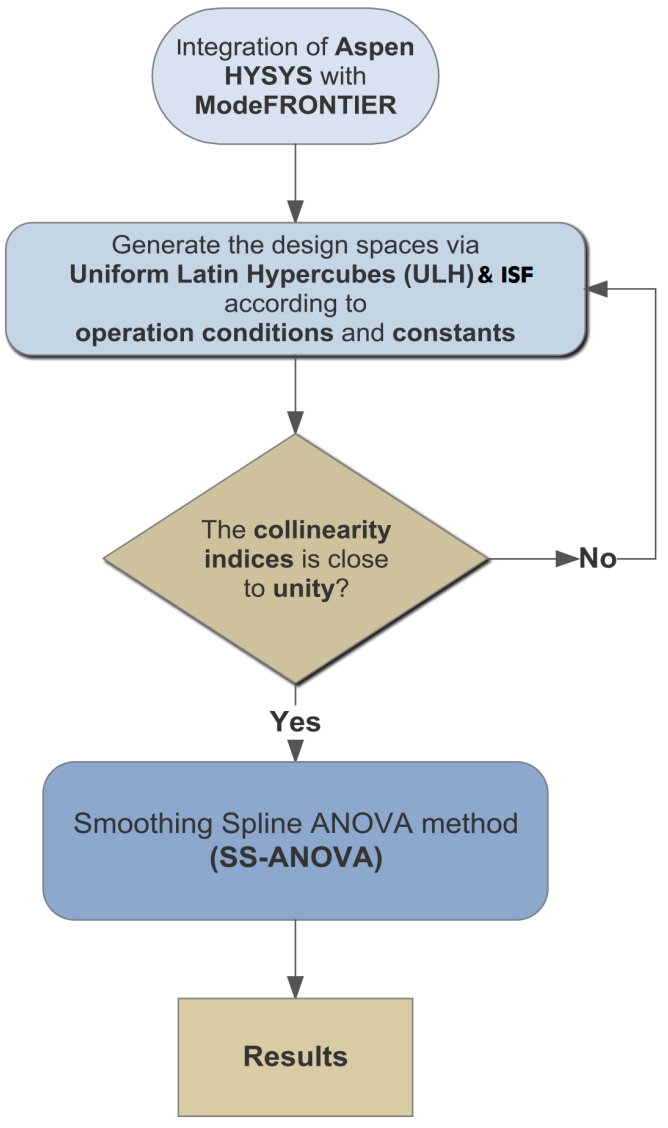

Source: Author

There is a tool to verify if the sample distribution of the selected input variables in design space was good sufficient. Scatter matrix chart is to see all the pairwise scatter plots of the input variables, linear dependence (correlation) between all the input variables and the Probability Density Function chart for each input variable. For example, Figure 4.21a shows the scatter matrix chart of the selected input parameter's distribution on the total fuel consumption of proposed FPSO. In that, each column and row represent a parameter. Squares with the number show the correlation of each variable and the other displays distribution points.

The Probability Density Function is practically uniform for all input variables and no correlation between the eight input variables is observed. Therefore, the design space can be considered satisfactory to perform the screening analysis of those eight input parameters.

Figure 4.21b shows an example distribution design point between P2 and P4. The green line represents the regression line that it is not observed any correlation between two input parameter P2 and P4. After ensuring from the initial distribution, Aspen HYSYS ${ }_{\cap}$ software is 
responsible to provide the numerical simulations. Then, the initial sampling of points is check by the maximum collinearity index until this index gets close to the unity. If not, new sampling points are added to the design space and the process continues until the collinearity index criteria is achieved.

Figure 4.21 - Initial population designs checking by statistical tools.

(a) Scatter matrix chart for screening analysis of the input parameters.

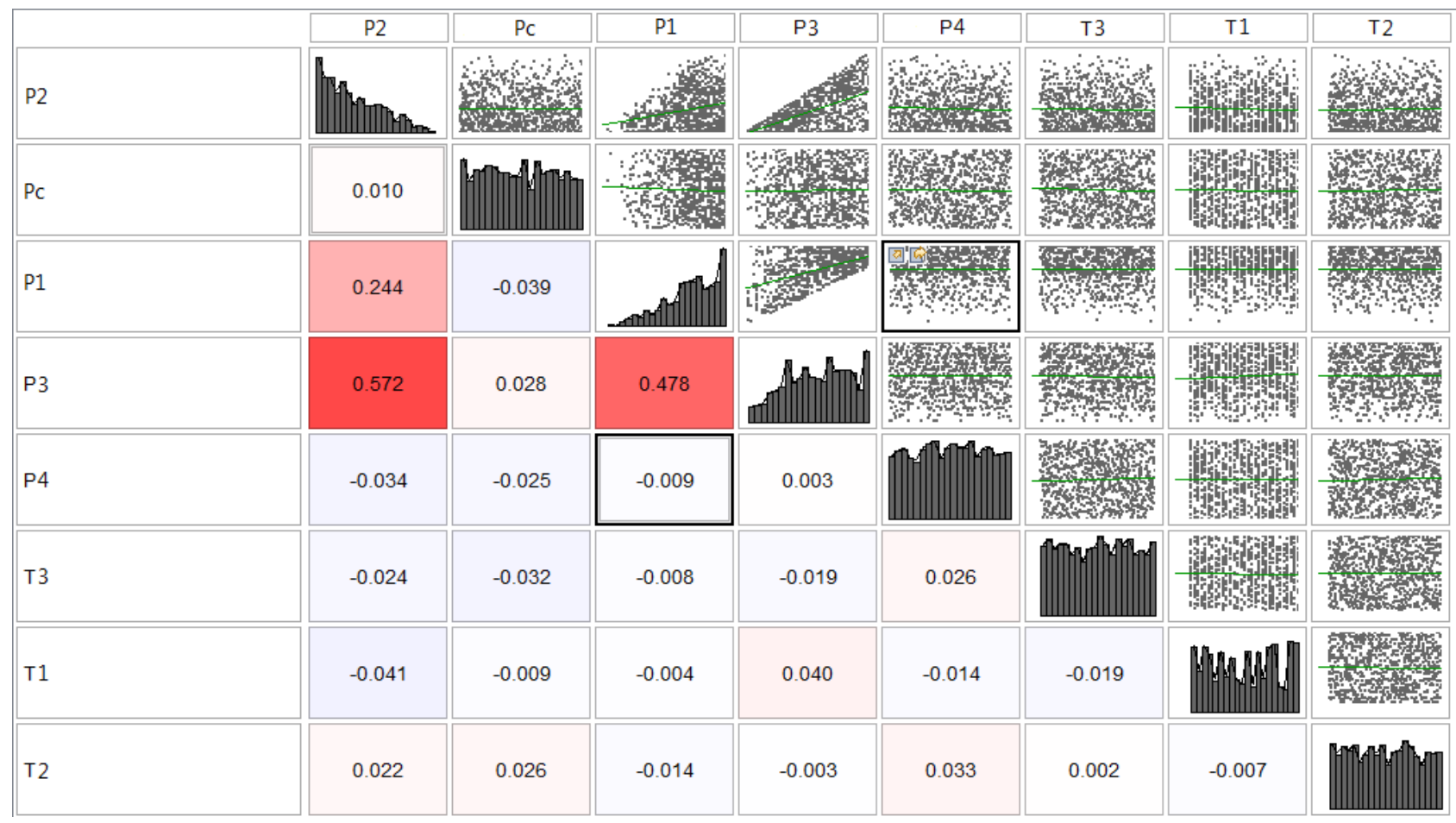

(b) linear dependence (correlation) between P2 and P4

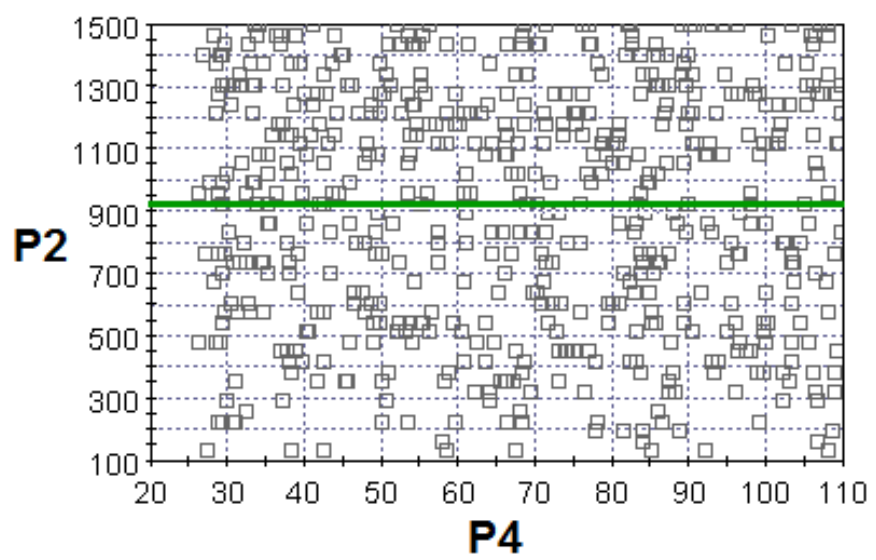

Source : Auhtor 


\subsubsection{Optimization description}

Optimization aims at defining the system configurations that, for example, simultaneously minimize the energy consumption or environmental impacts, while maximizing the thermodynamic performance (MARÉCHAL; KALITVENTZEFF, 1999).

The optimization problem is based on the decision variables, which can be changed in practice (for example, the output pressure of separator or the design temperature at the outlet of a heat exchanger), and on separation performance (subsection 3.1.1).

The objective function and design variables are firstly defined for the optimization procedure. The design space is then built by using Latin Hypercube Sampling and ISF (if necessary). In the next step, the Integrator software (modeFRONTIER) calls the thermodynamic solver (ASPEN HYSYS), which solves energy and mass balances for each component of the FPSO unit. After the thermodynamic convergence is achieved for the whole plant, the technical constraints are assessed. If the technical constraints are satisfied, values of Obj are picked up by integrator software. The optimization procedure is sought out by using NSGA-II. At the end of the optimization process, the Min or Max value of Obj calculates, if the convergence criteria fail, new points are generated and the procedure is restarted. The overall optimization procedure using the two programs (HYSYS and ModeFRONTIER ${ }^{\text {TM }}$ - details in Appendix C) is shown in Figure 4.22.

The objective function defined for the optimization procedure is a function of input parameters, their operating ranges and constraints:

$$
\text { Obj }=\text { Function }(\text { Input parameters })
$$

For example:

$$
\begin{gathered}
\text { Fuel Consumption }=f(\text { Input parameters }) \\
\text { Hydrocarbon liquids recovery }=f(\text { Input parameters })
\end{gathered}
$$

According to Rao (2009), defining a design vector $I$, the optimization problem can be stated as follows:

$$
I=\{\text { Inputs }\}
$$

Find $I=\{\ldots\}$ which minimizes/maximizes $O b j$ subject under constraints

$$
P_{i}>P_{i+1} \quad i=1,2
$$

It is important to note that all indicated technical constraints in the sensitivity analysis are also considered in the optimization procedure. The design of experiment of initial population 
of optimization procedure is also is verified by scatter matrix chart and collinearity induces.

Figure 4.22 - General flowchart of the optimization procedure

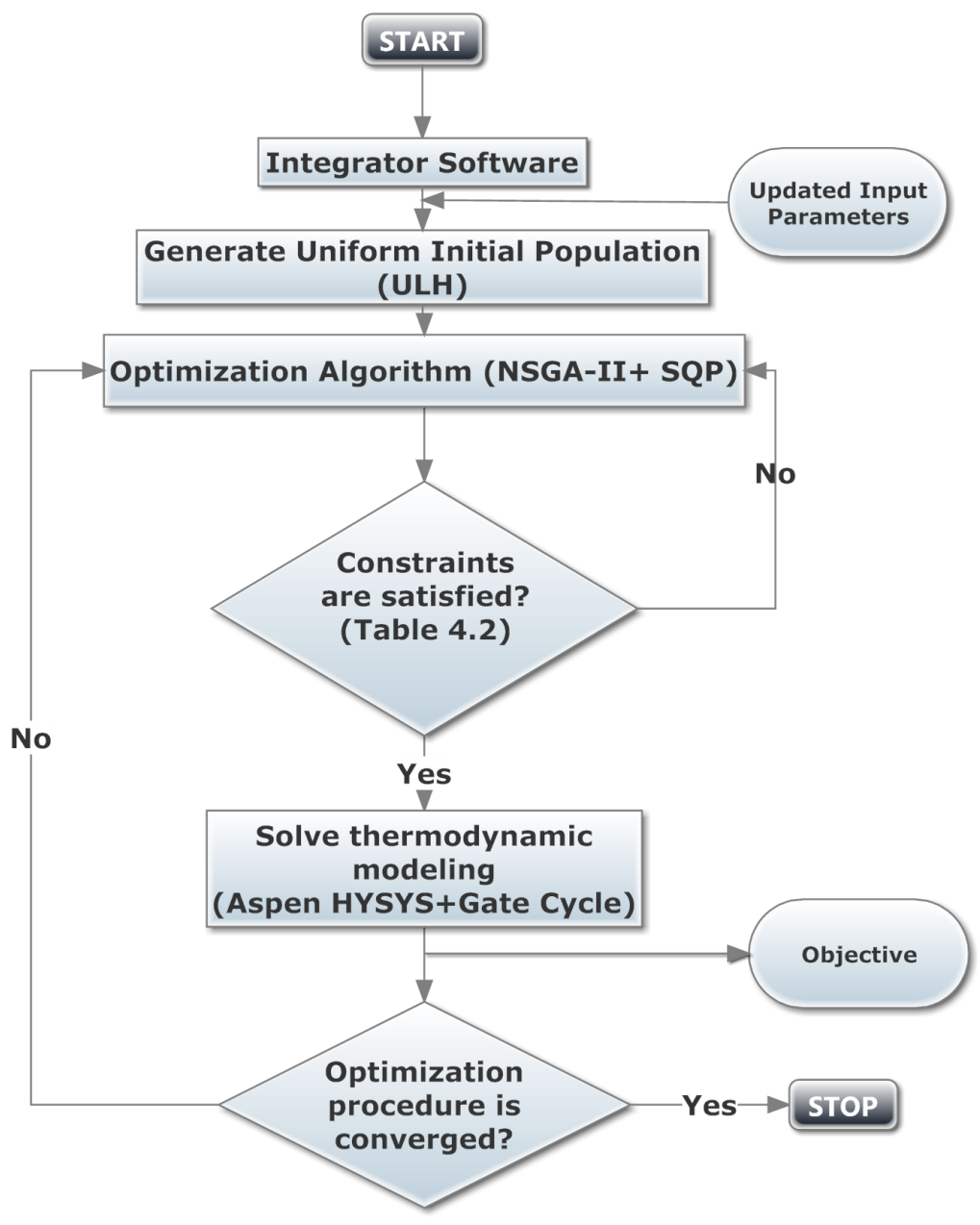

Source: Author

\subsubsection{Optimization in ModeFRONTIER ${ }^{\mathrm{TM}}$}

The ModeFRONTIER ${ }^{\text {TM }}$ is a multi-objective and multi-disciplinary optimization platform and design environment. Its workflow environment allows formalizing and managing all the logical steps composing an engineering process. The ModeFRONTIER ${ }^{\text {TM }}$ can be coupled with the popular engineering solvers or style tools available for building a bridge between ModeFRONTIER $^{\text {TM }}$ and any commercial or in-house codes. Figure 4.23 illustrates the procedure of optimization in ModeFRONTIER ${ }^{\mathrm{TM}}$. 
Figure 4.23 - The procedure of optimization in ModeFRONTIER ${ }^{\mathrm{TM}}$

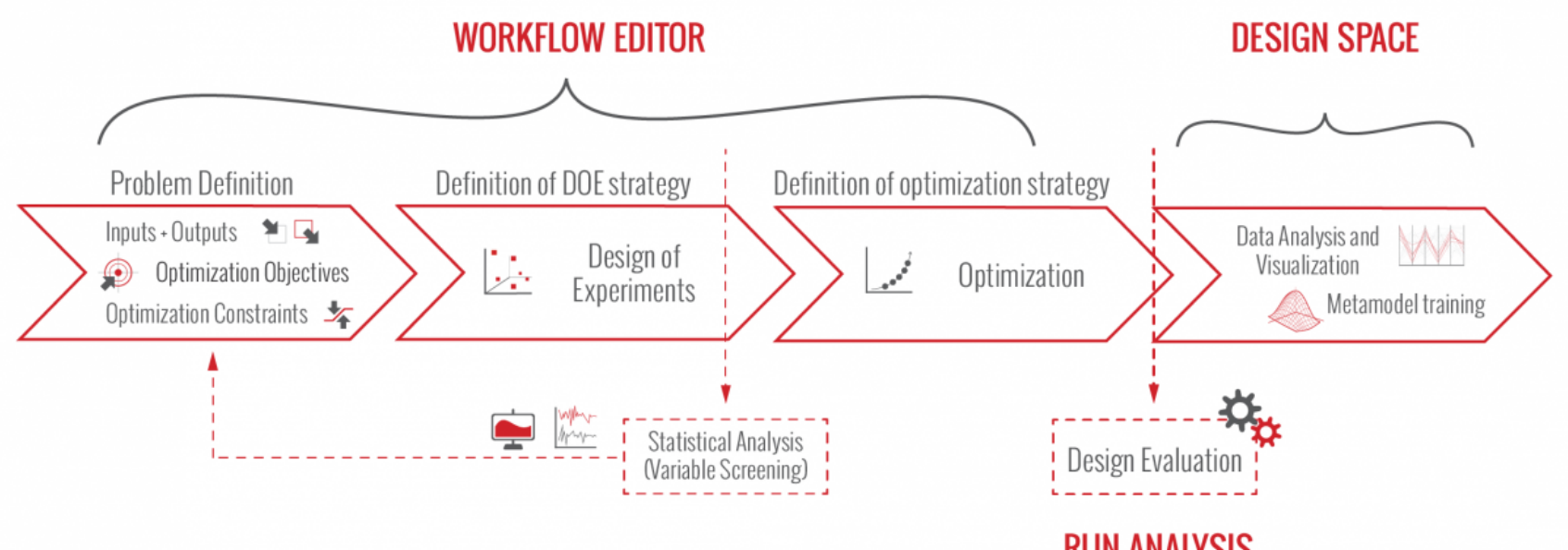

Source : https://academy.esteco.com/academy/training

ESTECO ModeFRONTIER ${ }^{\text {TM }}$ commercial code uses the Uniform Latin Hypercube algorithm that allows a regular equally-spaced sampling throughout the design space to attain the most information out of the points analyzed with the minimum number of design evaluations and it maximizes the minimum distance between neighboring points. One advantage of this software is that it uses a stochastic simulated annealing algorithm to generate a large number of candidate LH designs and chooses the one which best satisfies the maximum distance criterion.

Design of experiments based on the Latin Hypercube samplings and ISF is considered in this approach, one random generator seed as initial population. The some of considered assumptions are indicated for the optimization algorithm:

- The NSGA-II algorithms;

- Probability of 1 for Crossover;

- The mutation probability for real-coded vectors and for binary string is 1 ;

- Simple Crossover type for binary-coded variables.

The procedure will be run to converge the selected variable. The preliminary results are calculated according to the methodology implementation and are presented in the next chapter. 



\section{RESULTS AND DISCUSSIONS}

In this chapter, the obtained optimization results for a primary oil and gas processing plant in FPSO are presented and discussed. So that, a synthesis plant of typical Brazilian FPSO with three scenarios of operation is modeled and simulated by Aspen Hysys to calculate thermodynamic properties. The under investigation operation modes in the present research are: Mode 1, Maximum oil/gas content; Mode 2, 50\% BS\&W oil content and; Mode 3 , Maximum water $/ \mathrm{CO}_{2}$ oil content. The thermodynamic modeling and simulation of the proposed FPSO plant are performed using the real performance data of a commercial gas turbine (RB211G62 DLE 60Hz).

In addition, several thermodynamic parameters are submitted to a screening analysis via the Smoothing Splice ANOVA method to reduce the number of input variables for the optimization procedure. Finally, the main results from the optimization procedure via hybrid method for proposed objective functions are discussed and compared with a baseline configuration.

\subsection{Thermodynamic analysis results}

The FPSO plant model was used to investigate three operational modes shown in the Figures $4.14,4.15$ and 4.16, in order to obtain the required values of parameters, such as net oil, gas, water and $\mathrm{CO}_{2}$ production flows (mass balance) and energy balance. Table 5.1 shows the mass flow rates of the three studied operation modes. In that, as it was expected, the operation mode with more gas and oil (mode 1) has more (mass flow) production resulting in more required fuel for heat and power generation unit. Next, the operation mode 3 with more amounts of gas for injection than Mode 2, is in second place of the calculated electric power demand. Although, these two values are very close. Note that mode 1 has the highest gas mass flow rate and mode 2 has the lowest one, regarding the gas content of each crude oil and operating condition of separation train.

Moreover, as Mode 3 is maximum water $/ \mathrm{CO}_{2}$ oil content, the input mass flow rate of dilution water is the lowest and as Mode 1 is almost without water content, it has the highest of the necessary input mass flow rate of dilution water (Table 5.1). 
Table 5.1 - Mass flow rates of the three operational modes.

\begin{tabular}{cccc}
\hline Stream & $\begin{array}{c}\text { Mode 1 } \\
\text { mass flow }(t / h)\end{array}$ & $\begin{array}{c}\text { Mode 2 } \\
\text { mass flow }(t / h)\end{array}$ & $\begin{array}{c}\text { Mode 3 } \\
\text { mass flow }(t / h)\end{array}$ \\
\hline Crude Oil & 639.5 & 875.9 & 963.2 \\
Dilution Water & 48.7 & 22.4 & 9.9 \\
Air (Gas turbine) & 263.4 & 263.5 & 263.4 \\
Oil (Exportation) & 442.1 & 334.5 & 148.6 \\
Gas (Exportation) & 116.9 & 24.4 & - \\
Gas (Injection) & - & 27.5 & 76.1 \\
CO (Injection) & 63.2 & 37.3 & 9.1 \\
Gas for Fuel & 4.9 & 3.1 & 3.2 \\
Gas Exhaust & 268.4 & 266.6 & 266.6 \\
\hline
\end{tabular}

Both pressure and temperature in multistage crude oil separation processes play an important role in the efficiency of thermal systems and the separation of performance for processing plants. Table 5.2 and 5.3 show the operating pressures and temperatures of some streams in three operational modes. Because on injection, pressures of gas and $\mathrm{CO}_{2}$ injection processes are the highest of the overall process. Inlet of VRU has the minimum pressure (outlet of 1st of the separation process), while the outlet of MGC system refer to the compressed gas before the cooling and dehydration process.

Figure 5.1 shows the power demands for an FPSO plant corresponding to the energy consumed by pumps used for both water and oil recirculation and compressors for gas and $\mathrm{CO}_{2}$, for operation mode 1. In that, Main Gas Compression (MGC) has the highest power consumption due to the volume of gas and $\mathrm{CO}_{2}$ components to be compressed. Since all the gas produced is exported by Exportation Gas Compression (EGC), it is the second highest power consumption. The power consumption of $\mathrm{CO}_{2} \mathrm{C}$, which is responsible for preparing all the $\mathrm{CO}_{2}$ separated for injection, is the third highest power consumption. In the Vapor Recovery Unit (VRU), which is composed of two compressors at each stage, the compressor's shaft power is a function of the gas mass flow rate besides P1 and P3. Note that the power used in the separation train corresponds to the power consumed in the pumps used for water and oil. 
Table 5.2 - Operating pressures and temperatures of streams in the three operational modes.

\begin{tabular}{|c|c|c|c|c|c|c|c|}
\hline & & & Dilution & & & VRU & MGC \\
\hline & & Crude & Water & Oil & Water (cooling) & (inlet) & (outlet) \\
\hline & $\left({ }^{\circ} \mathrm{C}\right)$ & 19 & 28 & 73 & 20 & 85 & 40 \\
\hline & $(k P a)$ & 2300 & 600 & 441.4 & 900 & 243 & 8196 \\
\hline & $\left({ }^{\circ} \mathrm{C}\right)$ & 20 & 28 & 70 & 20 & 85 & 40.0 \\
\hline & $(k P a)$ & 2300 & 600 & 441.4 & 900 & 243 & 7944 \\
\hline & $\left({ }^{\circ} \mathrm{C}\right)$ & 20 & 28 & 70 & 20 & 85 & 40 \\
\hline 1VI0ue & $(k P a)$ & 2300 & 600 & 441.4 & 900 & 243 & 7288 \\
\hline
\end{tabular}

Table 5.3 - Operating pressures and temperatures of streams in the three operational modes.

\begin{tabular}{|c|c|c|c|c|c|}
\hline & & $\begin{array}{c}\text { Gas } \\
\text { (export) }\end{array}$ & $\begin{array}{c}\text { Gas } \\
\text { (injection) }\end{array}$ & $\begin{array}{l}\text { Gas } \\
\text { (fuel) }\end{array}$ & $\begin{array}{c}\mathrm{CO}_{2} \\
\text { (injection) }\end{array}$ \\
\hline \multirow{2}{*}{ Mode 1} & $\left({ }^{\circ} \mathrm{C}\right)$ & 40 & - & 37 & 40 \\
\hline & $(k P a)$ & 25047 & - & 4752 & 49500 \\
\hline \multirow{3}{*}{ Mode 2} & $\left({ }^{\circ} \mathrm{C}\right)$ & 40.0 & 40 & 37 & 40.0 \\
\hline & & & & & \\
\hline & $(k P a)$ & 24950 & 49500 & 4752 & 49450 \\
\hline \multirow{3}{*}{ Mode 3} & $\left({ }^{\circ} \mathrm{C}\right)$ & - & 40 & 37 & 40.0 \\
\hline & & & & & \\
\hline & $(k P a)$ & - & 49500 & 4752 & 49450 \\
\hline
\end{tabular}

Finally, MGC is responsible for $41 \%$ of the overall power consumption, followed by EGC is with $30 \%, \mathrm{CO}_{2} \mathrm{C}$ with $22 \%$, VRU with $4 \%$, Injection Gas Compression (IGC) with $1 \%$ and Separation Train (ST) with $1 \%$. 
Figure 5.1 - Power consumption [kW] and percentage for FPSO systems[\%] for operational mode 1.

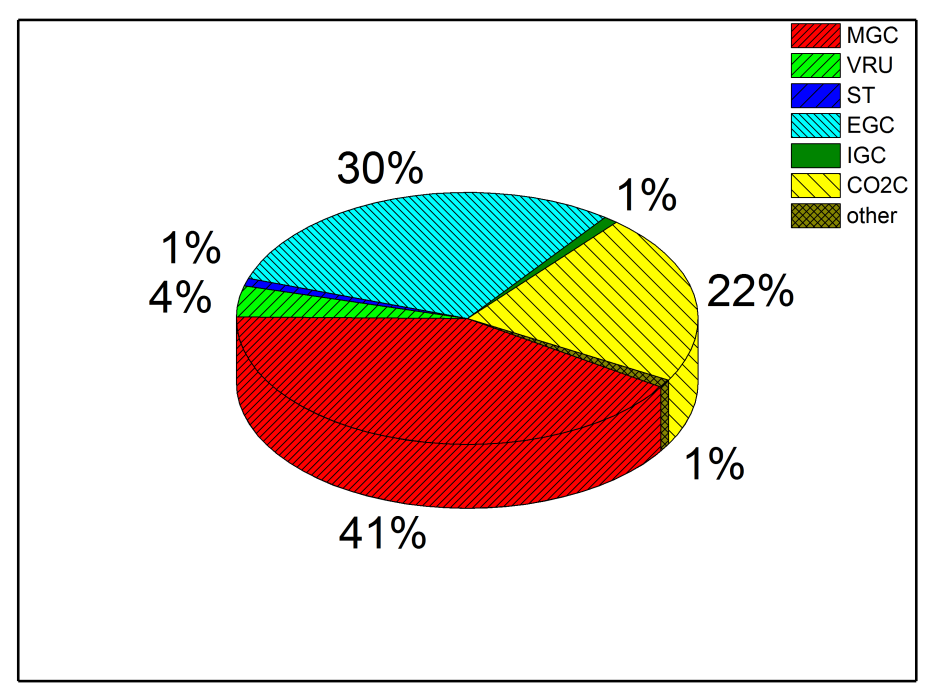

Source: Author

Figure 5.2 shows the power consumption for operation mode 2. In that, $36 \%$ of total power consumption is related to MGC, $10 \%$ to EGC, $24 \%$ to IGC. $23 \%$ to $\mathrm{CO}_{2} \mathrm{C}$, less of $1 \%$ to ST, $3 \%$ to $\mathrm{VRU}$ and more than of $3 \%$ to other systems. In this operation mode, $50 \%$ of the gas produced is injected; the total gas injection power requirement (both power demand in IGC section and in $\mathrm{CO}_{2} \mathrm{C}$ ) is thus higher than the power consumption of EGC (Figure 5.2).

Figure 5.2 - Power consumption [kW] and percentage for FPSO systems[\%] for operational mode 2.

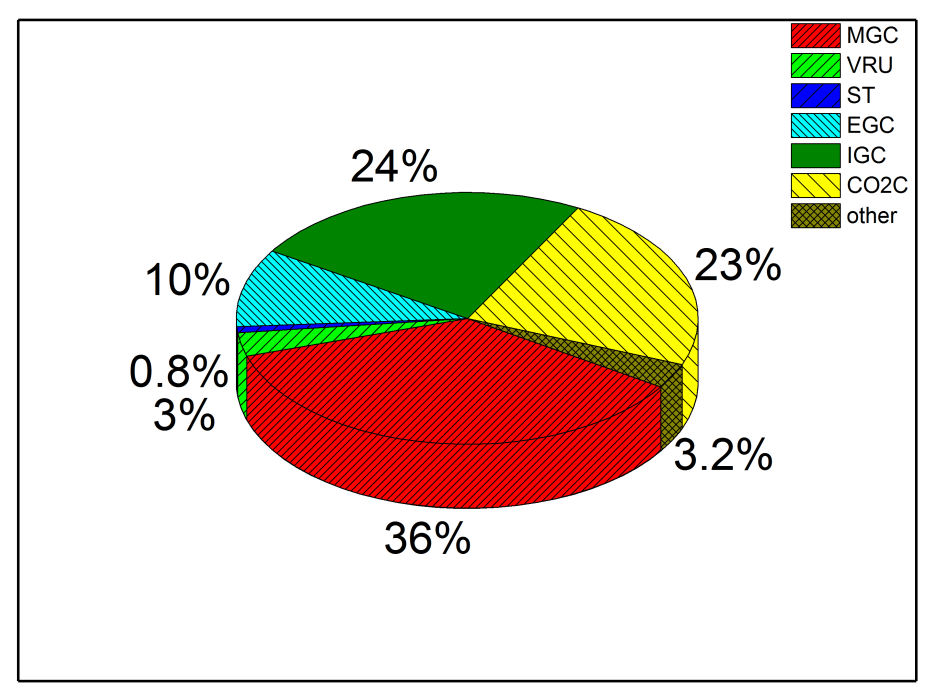

Source: Author 
For operation mode 3 , a great portion of the gas produced and all of the $\mathrm{CO}_{2}$ are injected. As shown in Figure 5.3, the effect of injection and compression (both natural gas and $\mathrm{CO}_{2}$ components) is greater. Therefore, $59 \%$ of total power consumption is for IGC (and $\mathrm{CO}_{2}$ ), $34 \%$ for MGC and $7 \%$ for the other sections.

Figure 5.3 - Power consumption [kW] and percentage for FPSO systems[\%] for operational mode 3.

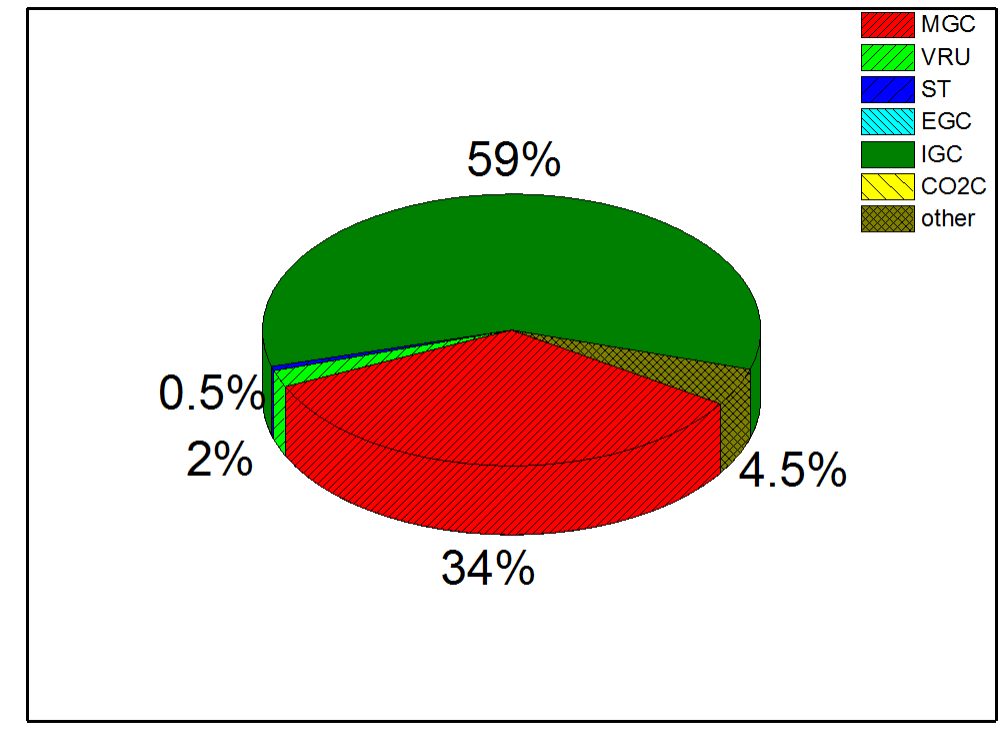

Source: Author

\subsection{Sensitivity analysis results}

The initial distribution of sampling points in design spaces was 50 for each operation mode. The number of designs was generated by ULH and ISF to meet the collinearity index criteria. The sampling points were increased regarding to each operation mode that is explained in their subsections. Thus, a sensitivity analysis of operating parameters in the recovery of hydrocarbon liquids and fuel consumption for the three different operation modes are performed to identify the most influential parameters.

\subsubsection{Contribution of Input Parameters on Hydrocarbon Liquids Recovery}

The primary objective of separation processes is to maximize recovery of hydrocarbon liquids that might otherwise flow into the gas stream and to remove dissolved gases from hydrocarbon liquids, increasing liquids production as well as its API gravity. On the other hand, stabilizing some hydrocarbon components such as pentanes plus in separation processes is necessary to reduce the volatility of crude oil and condensate. 
The most common manner used to remove the light components from hydrocarbon liquids before entering the liquid cargo tank is stage separation. It can be accomplished over simple stages of separation under suitable operating conditions. In this way, the hydrocarbon crude or condensate oil stream outs from the separator that usually flows through additional stages of separation or treatment before arriving at the sales point. In each of these stages, the liquid achieves near equilibrium at a different condition of pressure and temperature, thus to extent some "stabilizing" the crude or condensate (ARNOLD, 2007)\&(ABDEL-AAL et al., 2015).

\subsubsection{Operation mode 1}

As observed from Table 4.1, the fluid reservoir for operation mode 1 is a crude oil with maximum oil/gas content, which means that it has the highest oil production and fuel consumption as compared to the other operation modes. On the other hand, according to McCain et al. (2011), the crude oil composition of operation mode 1 is a volatile oil that has a lot of condensate components that are greatly influenced by the operational conditions, so that small variations in pressure and temperature may lead to the phase change of hydrocarbon components. Any change in the mass flow of exportation products, whatever gas phase products or liquid products, can affect the fuel consumption, leading to a difference in shaft work of pumps and compressors.

Furthermore, McCain et al. (2011) asserted that the type of reservoir fluid could be defined according to the composition of Heptane plus from a sample of reservoir fluid. Thus, according to data of operation mode 1 shown in Table 4.1, the Heptane plus that comprises $17.4 \%$ of the total composition should preferably be present in the oil stream. In addition, the available $\mathrm{C20}+$ in this reservoir fluid is a pseudo-component with the highest molar fraction in the exportation oil for all operation modes, which makes it a very important hydrocarbon component of Brazilian reservoirs.

For operation mode 1, 300 designs are generated by ULH and ISF considering the collinearity index criteria. Figure 5.4 shows the scatter matrix of input parameters for operation mode 1. In that, it can be seen the Probability Density Function is practically uniform for all input variables and it is not observed any correlation between 8 input variables. However, scatter matrix show a relation among $\mathrm{P} 1, \mathrm{P} 2$ and $\mathrm{P} 3$, but it is because of the considered constraints. As sequential pressure parameters must be lower than previous one, thus, regression line has sensed a connecting between these pressure. Nevertheless, the design space can be considered satisfactory to perform the screening analysis of those 8 input parameters in terms of fuel consumption and the recovery of hydrocarbon liquids. 
Figure 5.4 - Scatter matrix chart for screening analysis of the input parameters of operation mode 1.

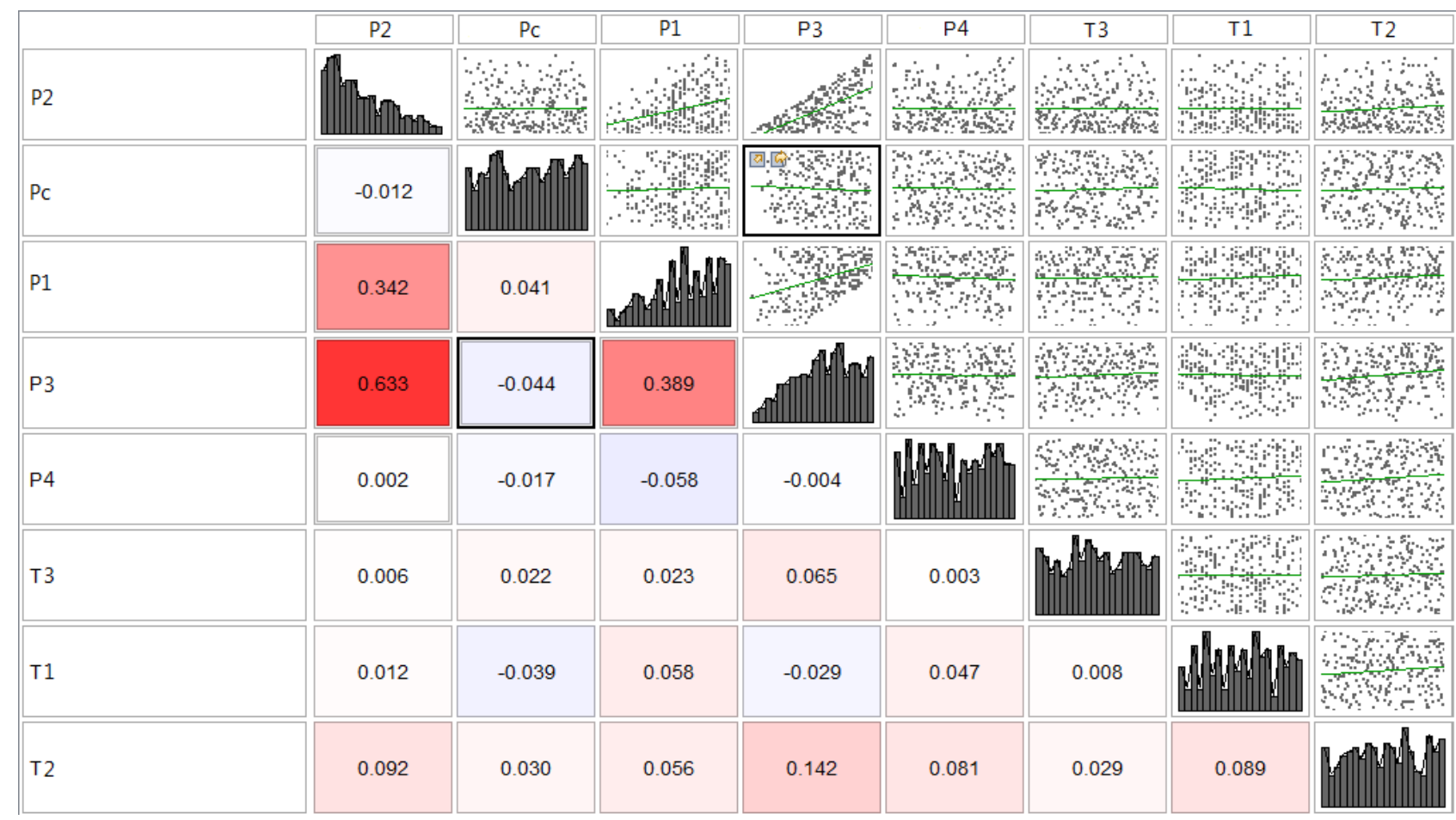

Source: Author

Figure 5.5 shows the effect of the input parameters on the hydrocarbon liquids recovery with maximum gas/oil content (operation mode 1). P3 is the most important contributor $(68 \%)$ to hydrocarbon liquids recovery, followed by P1 (13\%) and T2 (5\%). Moreover, a weak interaction effect is related to $P 1{ }^{*} \mathrm{P} 3$. The importance of $\mathrm{P} 3$ is related to the separation of heavy and pseudo-component hydrocarbon. Because of the proposed configuration of the separation train, the operating pressure of the third stage is also an important parameter to stabilize the volatile components in the liquid phase at a previous separation stage. P1 and T2 are important parameters for being related to the removal of light hydrocarbons from the crude oil and the recovery of intermediate hydrocarbons in the processed oil. However, as the petroleum composition is volatile, the impact of T2 compared to the pressure parameters is negligible. Therefore, the adequate condition for pressure plays a major role in determining the liquid amount of the recovered volatile hydrocarbons.

Consequently, these four input parameters (P1, P2, P3 and T2) correspond to 95\% of the total contribution to the recovery of hydrocarbon liquids for modes 1 . Thus, an optimization procedure for hydrocarbon liquid recovery maximization could be run with only these four input parameters, which reduces the time and computational resources, when compared to an optimization procedure with those eight initial input variables. 
Figure 5.5-Contribution of the input parameters to hydrocarbon liquids recovery for operation mode 1.

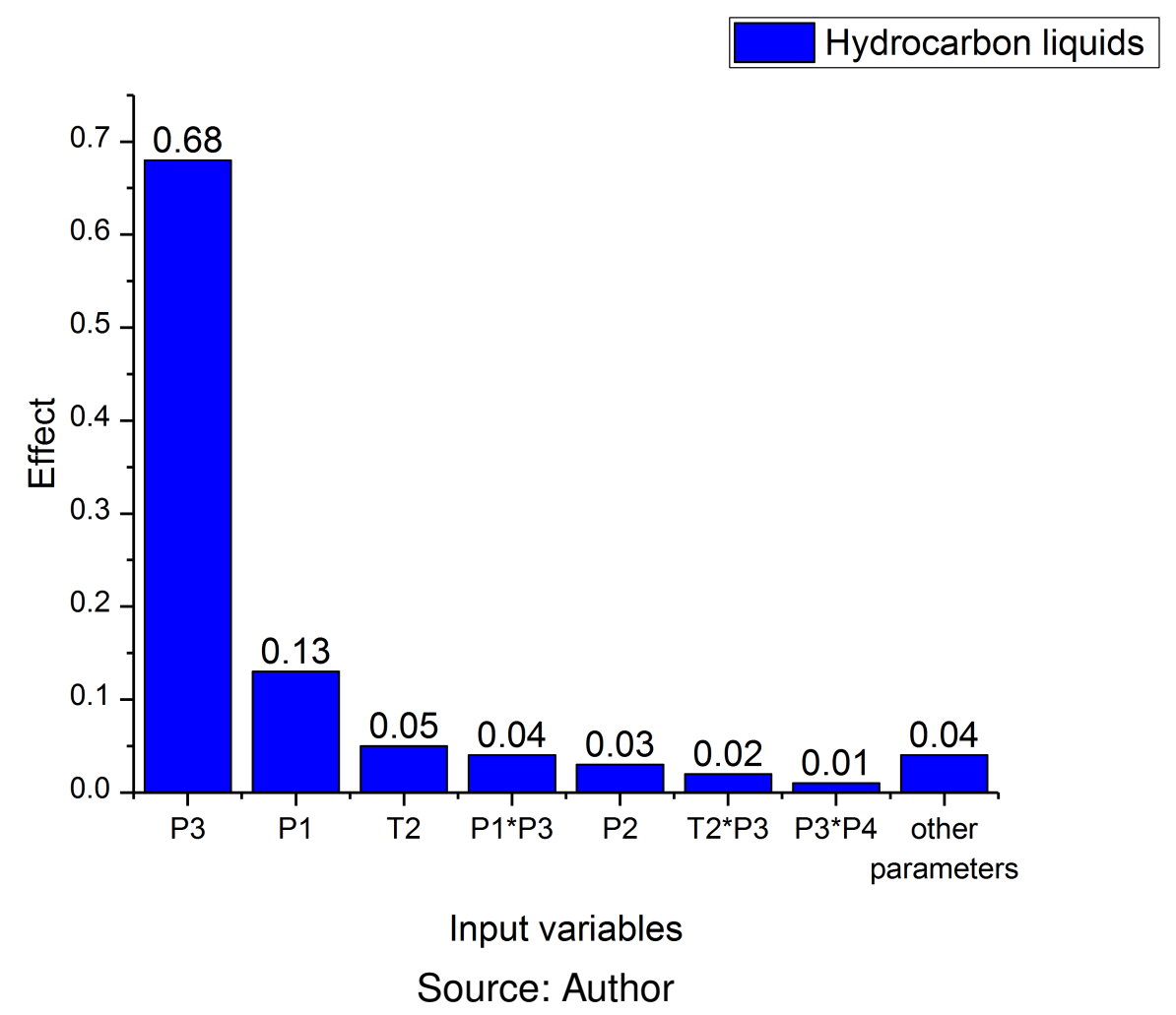

Figure 5.6 shows the separation efficiency for all stages of the separation train, in the conditions indicated in Table 5.4. The values of P1 and P3 presented in Table 5.4 are those that satisfy the processes constraints. The separation efficiency shows the significant effect of P3 as compared to other parameters. For condition $1\left(\mathrm{P} 1_{\max }=1999 \mathrm{kPa}, \mathrm{P} 3_{\max }=1050\right.$ $\mathrm{kPa})$ and condition $3\left(\mathrm{P}_{\text {max }}=1999 \mathrm{kPa}, \mathrm{P} 3_{\min }=101.5 \mathrm{kPa}\right)$, changing $\mathrm{P} 3$ from the upper limit to the lower limit makes the separation efficiency increase by more than $16 \%$.

On the other hand, for condition $3\left(\mathrm{P} 1_{\max }=1999 \mathrm{kPa}, \mathrm{P} 3_{\min }=101.5 \mathrm{kPa}\right)$ and condition 7 $\left(\mathrm{P} 1_{\text {min }}=605 \mathrm{kPa}, \mathrm{P} 3_{\text {min }}=101.5 \mathrm{kPa}\right)$, changing $\mathrm{P} 1$ from the upper limit to the lower limit affects the separation efficiency in less than $2 \%$. The influence of P3 is also analyzed as compared to the condition $1\left(\mathrm{P} 1_{\max }=1999 \mathrm{kPa}, \mathrm{P} 3_{\max }=1050 \mathrm{kPa}\right)$ to condition $2\left(\mathrm{P} 1_{\max }\right.$ $\left.=1999 \mathrm{kPa}, \mathrm{P} 3_{\text {design }}=243 \mathrm{kPa}\right)$, and condition $2\left(\mathrm{P} 1_{\text {max }}=1999 \mathrm{kPa}, \mathrm{P} 3_{\text {design }}=243 \mathrm{kPa}\right)$ to condition $5\left(\mathrm{P} 1_{\text {design }}=1300 \mathrm{kPa}, \mathrm{P} 3_{\text {design }}=243 \mathrm{kPa}\right)$.

The comparison of these operating conditions can help the design of the used separators, requirement of any additional heating for treatment, and storage tank. The storage tank from two terms can be evaluated (regarding the type of production). First one, weathering in a stock tank and second one, oil storage condition in case of operating issue and flashing critic components, such as propane and butane. 
Figure 5.6 - Total separation efficiency for the conditions shown in Table 5.4.

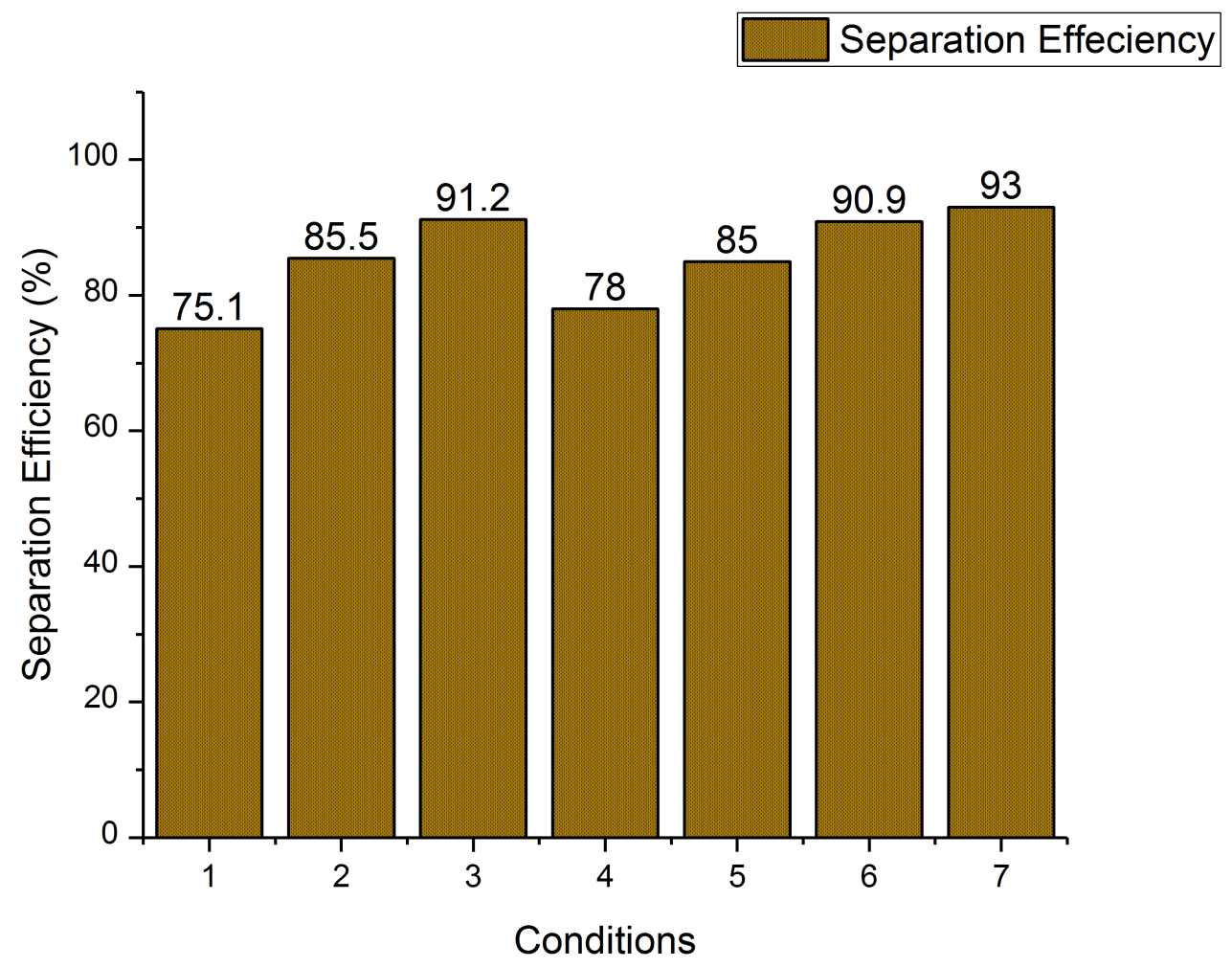

Source: Author

Table 5.4 - Hypothetic conditions to perform separation efficiency

\begin{tabular}{ccc}
\hline Conditions & $\mathbf{P 1}(\mathbf{k P a})$ & $\mathbf{P 3}(\mathbf{k P a})$ \\
\hline 1 & 1999 & 1050 \\
2 & 1999 & 243 \\
3 & 1999 & 101.5 \\
4 & 1300 & 790 \\
5 & 1300 & 243 \\
6 & 1300 & 101.5 \\
7 & 605 & 101.5 \\
\hline
\end{tabular}

Figure 5.7 shows the molar fraction of $\mathrm{C} 7+$ in the exportation oil for the conditions shown in Table 5.4. Similarly to Figure 5.6, the effect of P3 on C7+ is evident, yet the best condition for separation efficiency is not necessarily the best condition (condition 6) for the maximum stabilization of $\mathrm{C} 7+$. Considering the effect of the operating conditions on the intermediate hydrocarbon separation that occurs at the second stage of separation, it is possible to state that $\mathrm{P} 1=1300 \mathrm{kPa}$ is a better operating pressure for all phase of products rather than 605 $\mathrm{kPa}$. 
Figure 5.7 - C7+ molar fraction in exportation oil for the conditions shown in in Table 5.4.

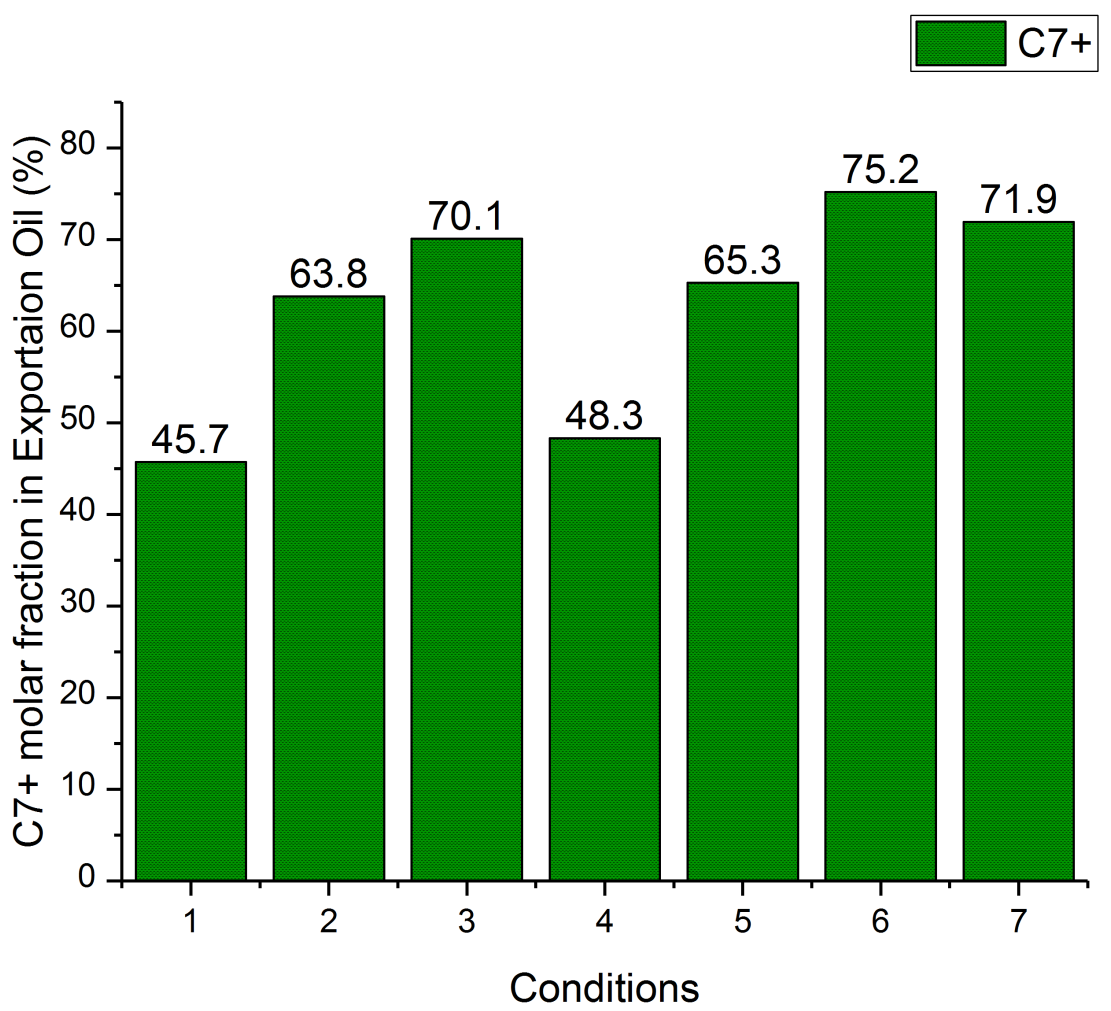

Source: Author

\subsubsection{Operation mode 2}

For operation mode 2, 860 designs are generated by ULH and ISF considering the collinearity index criteria. Figure 5.8 shows the scatter matrix of operation mode 2 for input parameters. In that, it can be seen in that the Probability Density Function is practically uniform for all input variables and it is not observed any correlation between the 8 input variables excepted $\mathrm{P} 1, \mathrm{P} 2$ and $\mathrm{P} 3$ that it is because of considered constraints (as also explained for mode 1).

According to Figure 5.9, for operation mode 2, the major contributor to hydrocarbon liquids recovery is also parameter $\mathrm{P} 3$ (about $50 \%$ of the total contribution), followed by T2 (37\%) and P2 (6\%). According to McCain et al. (2011), the composition of operation mode 2 is within the wet gas group $\left(0.5<Z_{C 7+}<4.5\right)$. Wet gas is a special two-phase flow (gas-liquid), which is often encountered in the oil and gas industry with the presence of hydrocarbons heavier than ethane, such as wet natural gas extraction from a condensate field (WANG, 2009). Moreover, the term "wet gas" is sometimes referred to gas condensate, which is sensitive to both operating pressure and temperature in separation conditions (TERRY; ROGERS, 2014; BAHADORI et al., 2013). Therefore, it can be concluded that parameters P3 and T2 are responsible (both main and interaction effects) for about $90 \%$ of the overall effect on hydrocarbon liquids recovery for operation mode 2. 
Figure 5.8 - Scatter matrix chart for screening analysis of the input parameters of operation mode 2.

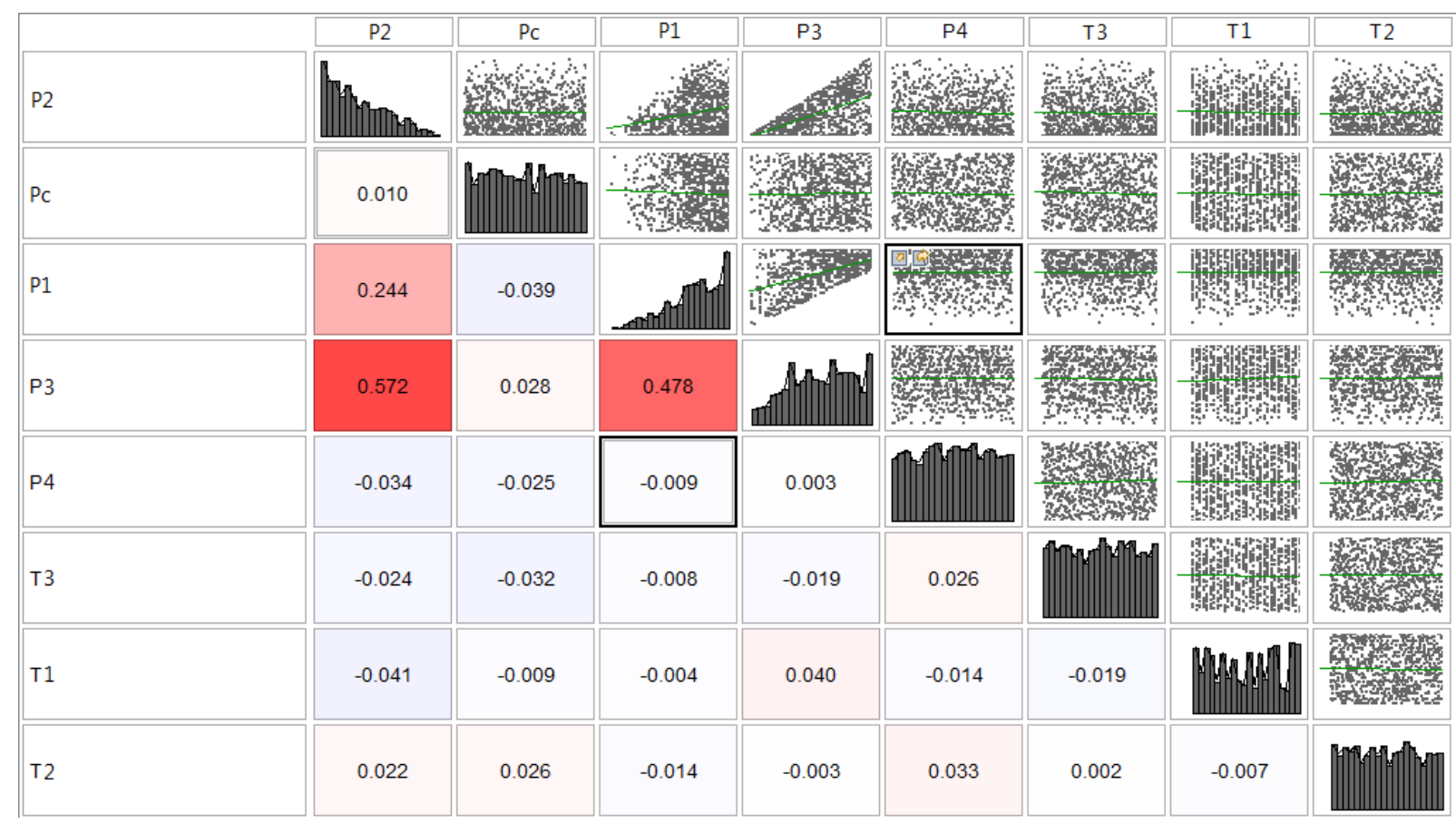

Source: Author

Figure 5.9-Contribution of the input parameters to hydrocarbon liquids recovery for operation mode 2.

Hydrocarbon liquids

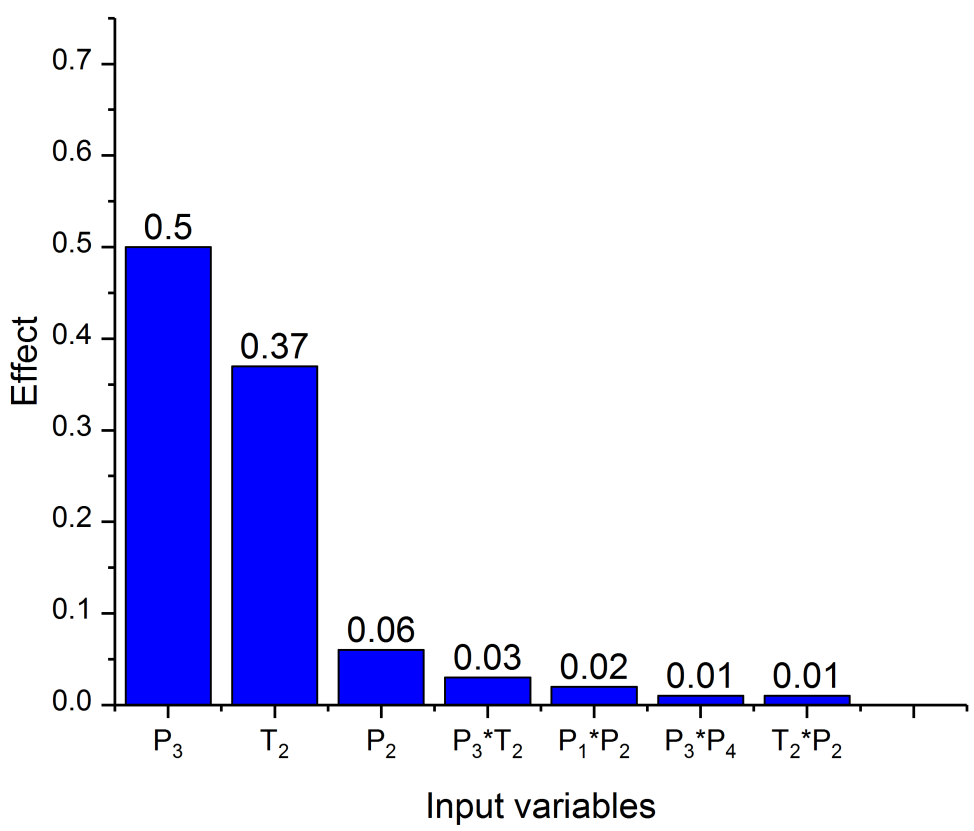

Source: Author 
The effect of P3 on the separation process is due to the presence of (C3-C5), C6+ and $\mathrm{C} 20+$ in the crude oil composition, and to the stabilization of those hydrocarbons in the last stage of the separation train. The effect of T2 can be attributed to the importance of the temperature variation in separation processes for a wet gas composition (as also a basic information was indicated in Figure 2.2).

In Table 5.5, different pressure operating conditions are investigated to understand the effects of those parameters on separation efficiency, when parameter $\mathrm{T} 2$ is varied from $80^{\circ} \mathrm{C}$ to $110^{\circ} \mathrm{C}$. Parameter $\mathrm{P} 1$ was kept constant and equal to $1650 \mathrm{kPa}$ in the four conditions. The results of the separation efficiency, based on the pressure conditions shown in Table 5.5, are reported in Figure 5.10. The separation efficiency in condition 1 is about $30 \%$ higher than that presented for condition 2 at a temperature of $80^{\circ} \mathrm{C}$, while from condition 2 to condition 4 , the separation efficiency is almost independent of the P2 variation. When T2 changes from $80^{\circ} \mathrm{C}$ to $110^{\circ} \mathrm{C}$, at the same pressure operating condition, a separation efficiency increase of $3.7 \%, 5.6 \%, 7.1 \%$ and $5.6 \%$ is observed for conditions $1,2,3$ and 4 , respectively.

Table 5.5 - Different pressure operating conditions to perform separation efficiency

\begin{tabular}{cccc}
\hline Conditions & P1(kPa) & P2(kPa) & P3(kPa) \\
\hline 1 & 1650 & 1050 & 1040 \\
2 & 1650 & 1050 & 101.5 \\
3 & 1650 & 1050 & 605 \\
4 & 1650 & 440 & 101.5 \\
\hline
\end{tabular}

Figure 5.10 - Total separation efficiency for the conditions shown in Table 5.5.

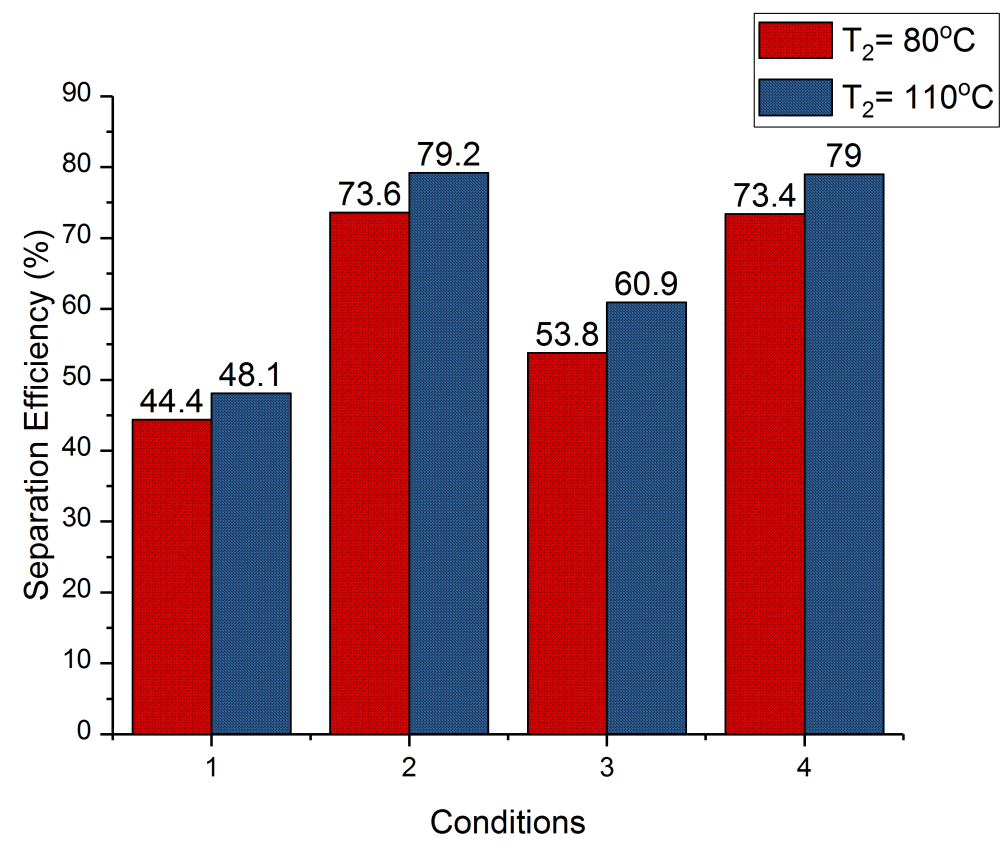

Source: Author 


\subsubsection{Operation mode 3}

For operation mode 3, 1500 designs are generated by ULH and ISF. Figure 5.11 shows the scatter matrix of operation mode 3 for input parameters. The Scatter Matrix chart shows whether there is any linear dependence (correlation) between two variables and the nature and the strength of such correlation, how data is dispersed and if any anomalies are present, and whether data has some kind of a recognizable distribution. The appeared red color, is also because of the defined constraints between input parameters.

Figure 5.11 - Scatter matrix chart for screening analysis of the input parameters of operation mode 2.

\begin{tabular}{|c|c|c|c|c|c|c|c|c|}
\hline & P2 & Pc & P1 & P3 & P4 & T3 & $\mathrm{T} 1$ & T2 \\
\hline P2 & & 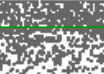 & & 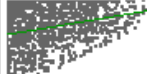 & 是: & , & 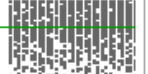 & \\
\hline Pc & -0.006 & & & $4 x$ & & 竞 & $y^{3}$ & \\
\hline P1 & 0.478 & 0.003 & & & & & 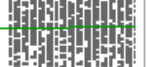 & \\
\hline P3 & 0.264 & -0.022 & 0.567 & 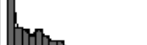 & & & & \\
\hline P4 & 0.001 & -0.025 & -0.015 & -0.005 & & & & \\
\hline T3 & 0.018 & 0.024 & -0.009 & -0.014 & 0.042 & & & \\
\hline T1 & -0.005 & 0.010 & 0.005 & -0.011 & 0.020 & -0.011 & MH & \\
\hline T2 & 0.000 & 0.011 & 0.020 & 0.040 & 0.041 & -0.000 & 0.003 & \\
\hline
\end{tabular}

Source: Author

Figure 5.12 shows the effects of the input parameters on hydrocarbon liquids recovery for operation mode 3 (Maximum water/ $\mathrm{CO}_{2}$ oil content). As can be seen, the composition of hydrocarbon components for this operation mode 3 is similar to the operation mode 2; thus, the contribution of each parameter for operation mode 3 is very close to which is presented for the operation mode 2. In this case, only P3 and T2 present a significant effect on hydrocarbon liquids recovery, reaching about $87 \%$ of the total effect. The $85 \%$ of total effect is from main effect and $2 \%$ is coming from interaction effect.

The effect of operating temperature (T2) as a specific treatment for wet gas composition is shown in results of Figure 5.12. In addition, as Figure 5.12 displays, the importance of 
operating pressure of the last separation stage (P3) on the recovery and stabilization of volatile components of wet gas is about half of the total effect.

Figure 5.12 - Contribution of the input parameters to hydrocarbon liquids recovery for operation mode 3.

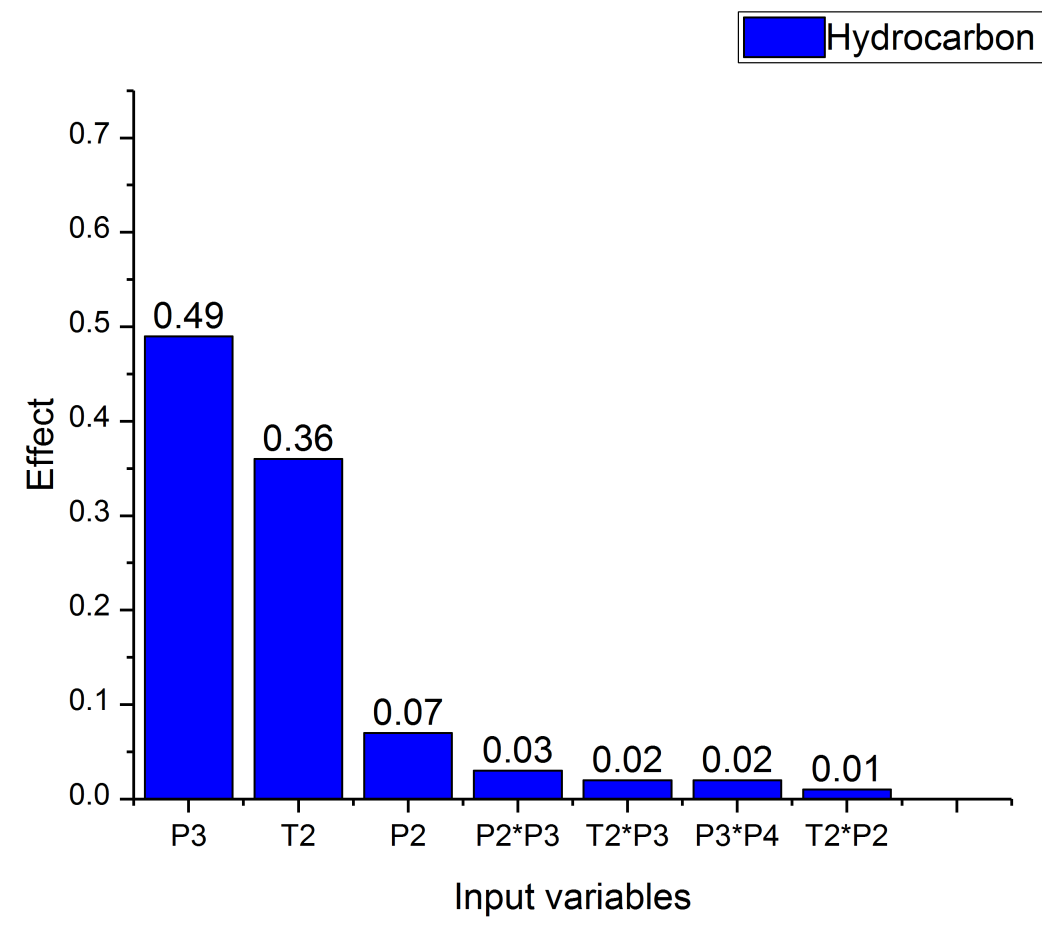

Source: Author

Overall, the comparison of the three operation modes indicates that P3 is the most important contributor to hydrocarbon liquids recovery and its order of magnitude is quite different for mode 1 , mode 2 and 3 . The second most important contributor for hydrocarbon liquids recovery is $\mathrm{P} 1$ for mode 1 and T2 for modes 2 and 3 . In terms of P3 and T2 parameters, the contribution of P3 is about $63 \%$ higher than T2 for mode 1 and $13 \%$ higher for modes 2 and 3. It is also important to note that the contribution of P2 can be considered negligible for mode 1, but important for modes 2 and 3 (for these 2 modes there are small interactions between P2 and other parameters which must be added to the overall contribution).

From the previous discussion on the maximization of hydrocarbon liquids recovery in separation stages, based on the proposed operating conditions, the parameters that should be taken into account for an optimization procedure are: P3 and T2 (for all operation modes), P1 (only for operation mode 1) and P2 (for operation modes 2 and 3). Thus, by reducing the number of input parameters, the optimization procedure becomes simpler and faster, which is an important advantage to save time and computational resources (Table 5.6). 
Table 5.6 - Input parameters and their respective operating ranges to be used in the maximization of hydrocarbon liquids recovery

\begin{tabular}{ccc}
\hline & $\begin{array}{c}\text { Input } \\
\text { parameter }\end{array}$ & $\begin{array}{c}\text { Operation } \\
\text { range }\end{array}$ \\
& $P 1$ & $101.3-2000 \mathrm{kPa}$ \\
Mode 1 & $P 2$ & $101.3-1050 \mathrm{kPa}$ \\
& & $101.3-1050 \mathrm{kPa}$ \\
& T2 & $30-110^{\circ} \mathrm{C}$ \\
& & \\
\hline & & $101.3-450 \mathrm{kPa}$ \\
& P3 & $101.3-1050 \mathrm{kPa}$ \\
& P2 & $30-110^{\circ} \mathrm{C}$ \\
& T2 & \\
\hline & & $101.3-450 \mathrm{kPa}$ \\
& P3 & $30-110^{\circ} \mathrm{C}$ \\
\hline
\end{tabular}

\subsubsection{The contribution of Input Parameters To Fuel Consumption}

As explained previously, regarding reservoir hydrocarbon composition, operation mode 1 is the highest fuel consumption mode. Thus, due to its importance as compared to the other operation modes, a suitable value of input parameters of operating conditions affect the total fuel consumption and eventually lead to a more profitable system. Firstly, to explain the results from this section, it is necessary to analyze the effect of input parameters on the power demand. Mass flow and output pressure of compressors and pumps are parameters that increase the power demand and fuel consumption whereas their isentropic efficiencies remain slightly constant.

\subsubsection{Operation mode 1}

Figure 5.13 shows the main and interaction effects contribution of input parameters on fuel consumption for operation mode 1 . The results indicate that parameters P3, P1 and Pc are the major contributors to power consumption and, consequently, to fuel consumption, corresponding to about $81 \%$ of the total effect(their main effects). Parameter P3 is the major contributor and its main effect on fuel consumption is $10 \%$ higher than that of P1 and Pc. P2 is also an important parameter that impacts fuel consumption since its main effect is about $6 \%$ of the total variance. Moreover, the main effects were more significant than the interaction effects on fuel consumption, corresponding to $87 \%$ of the overall variance, similarly to that 
also observed for hydrocarbon liquids recovery.

Figure 5.13 - Contribution of input parameters to fuel consumption in mode 1.

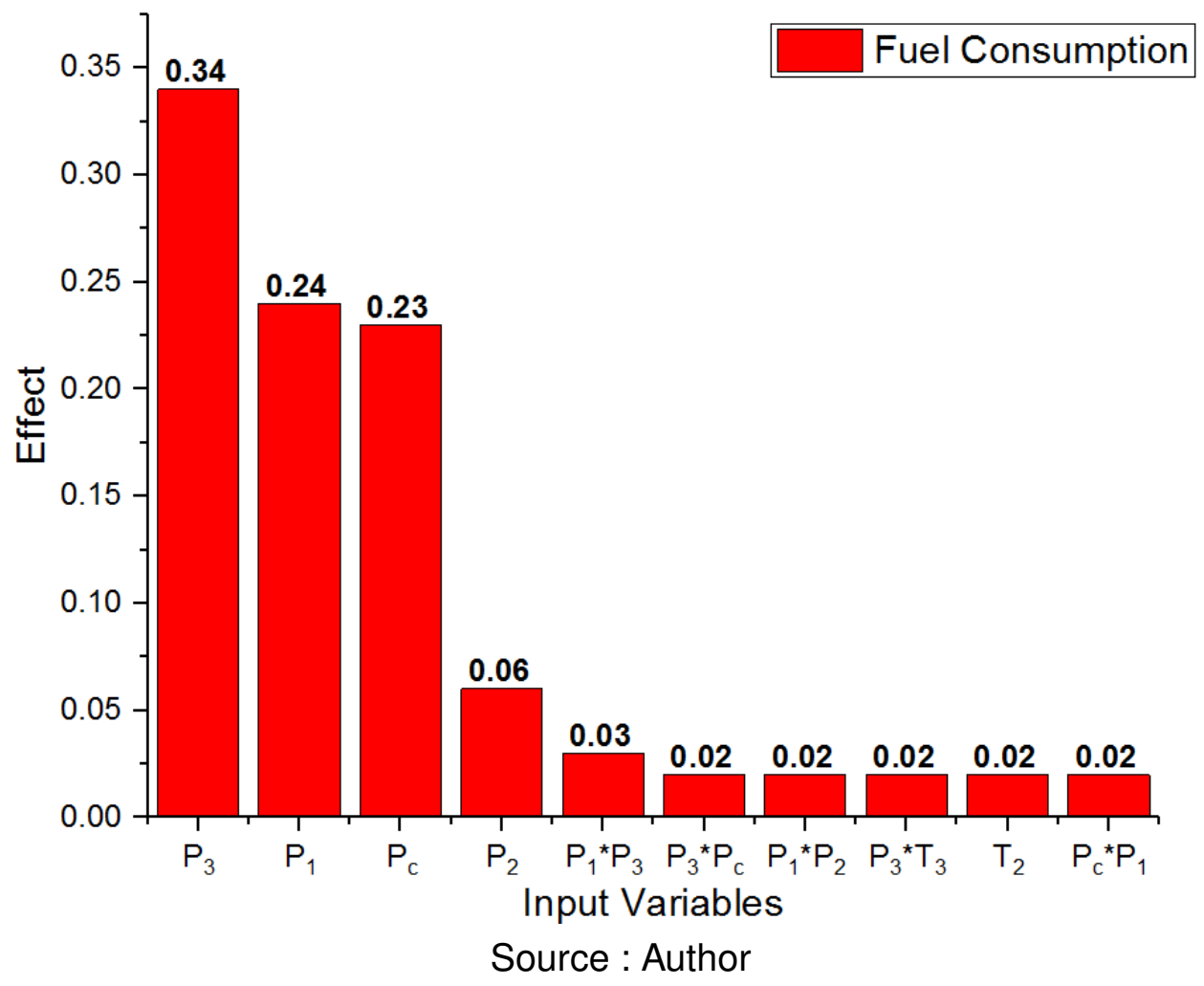

The effect of both P1 and P3 on compressors of the VRU could be explained by a greater pressure difference between the compressor sides, which leads to a greater power consumption due to the compressors of the VRU. However, from Figure 5.1, the power demand of VRU is only $4 \%$ of all the power consumption (for a middle-range condition); thus, the difference between the pressure on the permeate and retentate sides could not be the single reason for their effect on fuel consumption. On the other hand, as the quantity of light hydrocarbon components in crude oil is high for this operation mode, P1 is expected to be the most important parameter in fuel consumption. Therefore, the input parameters of the second stage of the separation train can impact the gas phase separation and, consequently, more gas is sent to the first stage of VRU, increasing the fuel consumption of compressors. However, from the results, its main effect is responsible for only $24 \%$ of the total effects. Besides the impact on the compressor shaft work of the second stage of the vapor recovery unit (output pressure effect), P3 also affects the mass flow of exportation oil because of the recovery of hydrocarbon liquids and the stabilization of the resultant phases (gas and liquid) of production leaving the third separation train (Figure 5.5, Figure 5.6 and Figure 5.7). Hence, P3 is the most important contributor to fuel consumption. The next influential parameter is $\mathrm{P} 1$, which affects the separation efficiency, as well as the compressor power of 
the first stage of the vapor recovery unit. Note that besides the output pressure, both mass flows from the separation train to VRU and then from the VRU compressors to MGC (as the highest power consumption unit) have a great impact on the required power. Therefore, if the maximum recovery of hydrocarbon liquids is not accomplished, a larger amount will be lost to the processed gas and sent to gas treatment sections resulting in an increase of power consumption. For inlet parameter $\mathrm{Pc}$, it is obvious that when $\mathrm{Pc}$ increases, a higher compressor shaft work is required to achieve the necessary output pressure. The influence of $2 \%$ on fuel consumption due to T2 is small and can be explainable by its influence on the stabilization of volatile components. Finally, P1, P3 and Pc are responsible for about $88 \%$ of the total input parameters impact on fuel consumption.

\subsubsection{Operation mode 2}

In operation mode 2, $50 \%$ of the gas produced is injected; the total gas injection power requirement (both power demand in IGC section and in $\mathrm{CO}_{2} \mathrm{C}$ ) is thus higher than the power consumption of EGC as shown in Figure 5.14. The influence of input parameters on fuel consumption for operation mode 2 is presented in Figure 5.14. The contribution of input parameter P3 is the highest (63\%) followed by Pc (17\%), T2 (16\%) and P2 (4\%). The effect of P3 on fuel consumption is $45 \%$ higher than Pc and T2. Relevant interaction effects between the input variables are not observed. The T2 effect, as explained before, is due to the separation process for intermediate and heavy hydrocarbons in the stabilization phase of wet gas (Figures 5.9, 5.10).

Figure 5.14 - Contribution of input parameters to fuel consumption in mode 2.

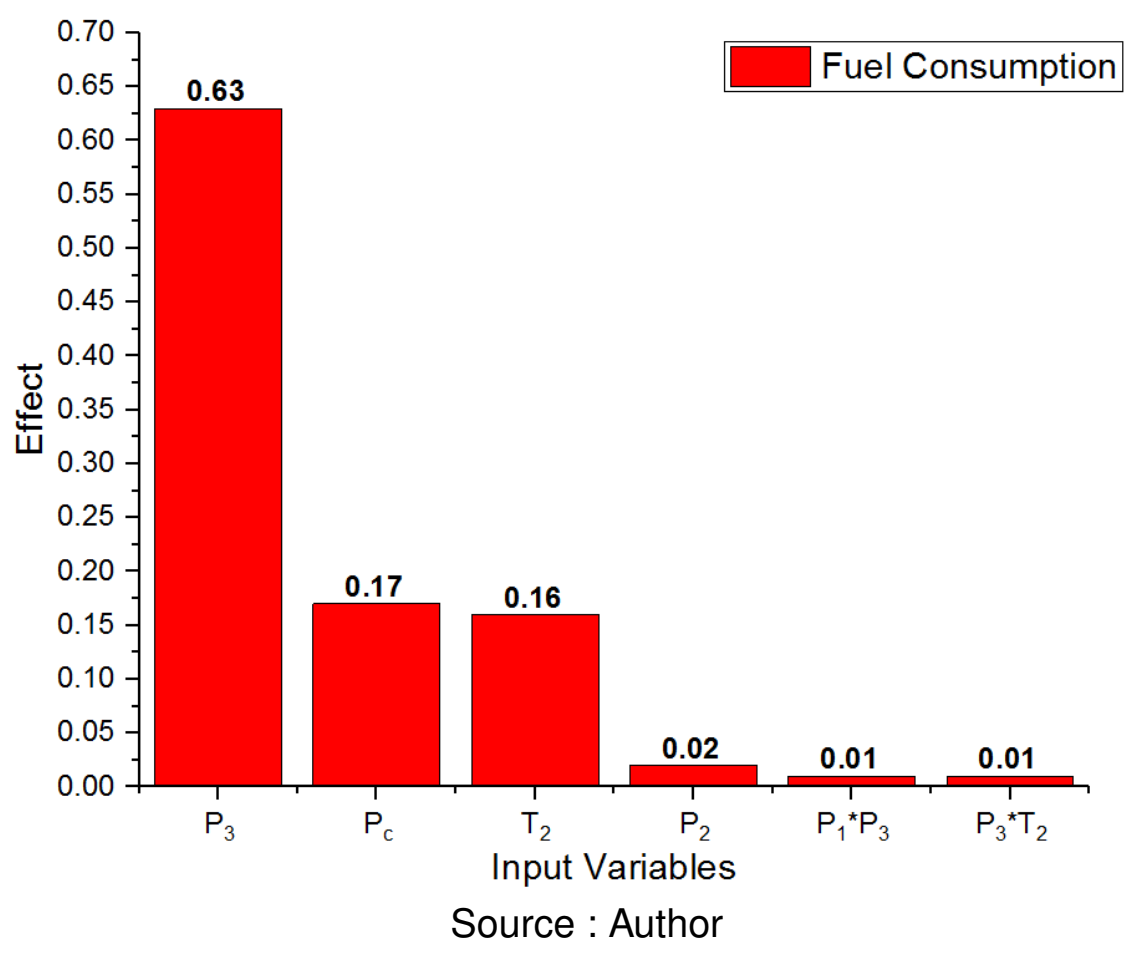


The effect of Pc on fuel consumption is smaller than that of operation mode 1 due to the lower concentration of $\mathrm{CO}_{2}$ and light hydrocarbon components (Figure 5.13). The effect of $\mathrm{P} 3$ is due to the separation of $\mathrm{C} 7+$ components and intermediate hydrocarbons (as shown in Figure 5.10) and also its effect on the VRU compressor. The contribution of both P3 and PC to fuel consumption is about $80 \%$ of the total impact for mode 2 . As the gas content is not high in mode 2, parameter $\mathrm{P} 1$ is relevant only for operation mode 1 and can be considered negligible for operation mode 2 . Hence, P3, Pc and T2 are responsible for $97 \%$ of the total impact.

\subsubsection{Operation mode 3}

Figure 5.15 shows that Pc, P3 and T2 are the most important contributors to fuel consumption for operation mode 3. The effect of the Pc input parameter is verified to be about $50 \%$ of the total variance due to the highest $\mathrm{CO}_{2}$ mass flow for this mode. Parameter T2 is relevant for both modes 2 and 3 and its contribution is practically the same for both operation modes. Finally, an unusual interaction is observed between T2* P3, corresponding to $10 \%$ of the total variance. This interaction is due to the recovered wet gas components through the third stage of the separation train (P3).

Figure 5.15 - Contribution of input parameters to fuel consumption in mode 3.

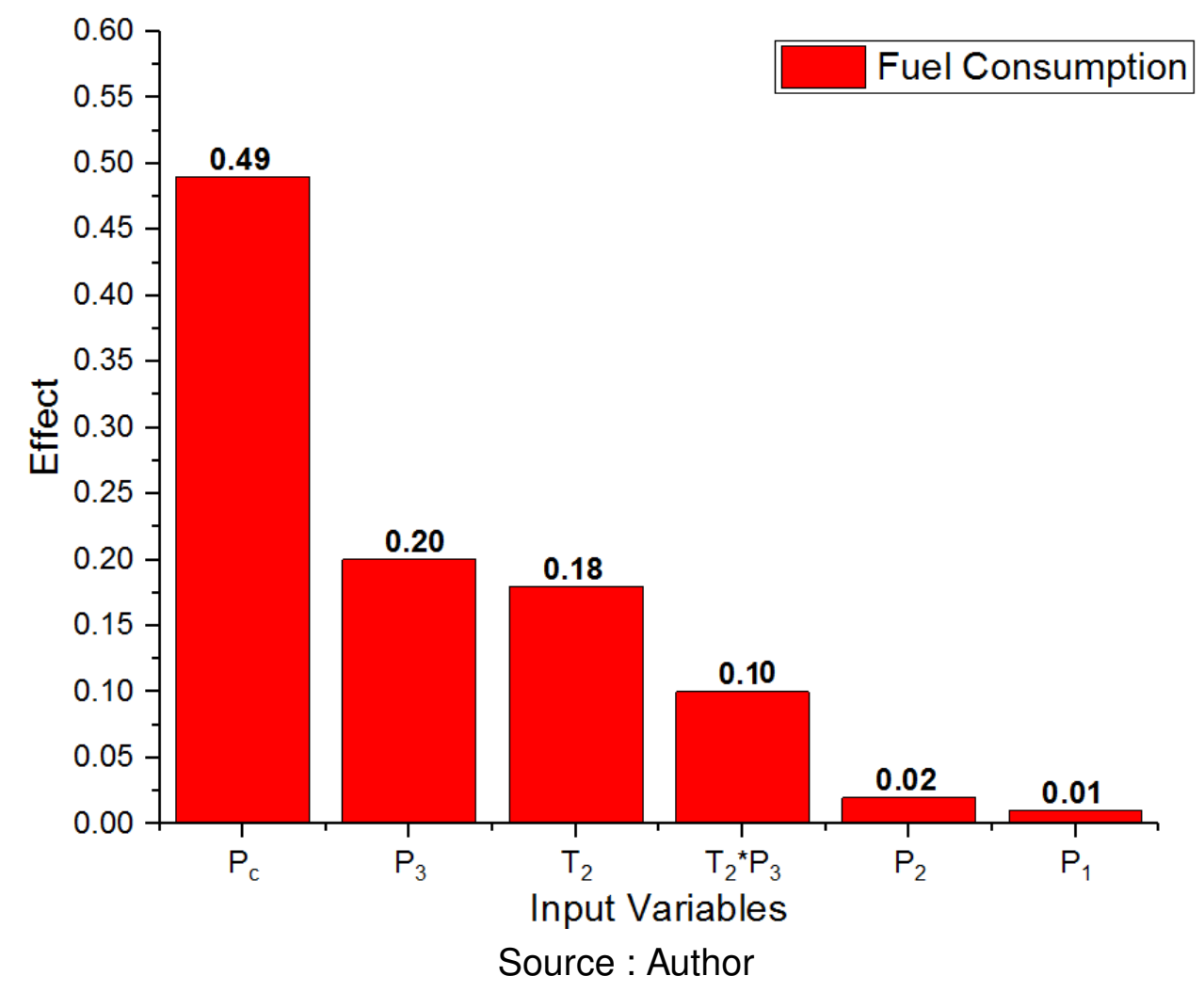

From SS-ANOVA, the statistical analysis revealed that the most important parameters 
affecting the fuel consumption of the plant for three operation mode are shown in Table 5.7 , these most effective of input parameters correspond to $96 \%, 97 \%$ and $97 \%$ of the total contribution to fuel consumption for modes 1, 2 and 3, respectively. Thus, an optimization procedure for fuel consumption minimization could be run with only these input parameters, which reduces the time and computational resources, when compared to an optimization procedure with those eight initial input variables.

Table 5.7 - Input parameters and their respective operating ranges to be used in the optimization procedure of fuel consumption minimization

\begin{tabular}{|c|c|c|}
\hline & $\begin{array}{c}\text { Input } \\
\text { parameter }\end{array}$ & $\begin{array}{c}\text { Operation } \\
\text { range }\end{array}$ \\
\hline \multirow{4}{*}{ Mode 1} & P1 & $101.3-2000 \mathrm{kPa}$ \\
\hline & P2 & $101.3-1050 \mathrm{kPa}$ \\
\hline & P3 & $101.3-1050 \mathrm{kPa}$ \\
\hline & Pc & $7018-8500 \mathrm{kPa}$ \\
\hline \multirow{4}{*}{ Mode 2} & P3 & $101.3-450 \mathrm{kPa}=\mathrm{P} 2$ \\
\hline & Pc & $7018-8500 \mathrm{kPa}$ \\
\hline & $\mathrm{T} 2$ & $30-110^{\circ} \mathrm{C}$ \\
\hline & P3 & $101.3-450 \mathrm{kPa}=\mathrm{P} 2$ \\
\hline \multirow[t]{2}{*}{ Mode 3} & Pc & $7018-8500 \mathrm{kPa}$ \\
\hline & $\mathrm{T} 2$ & $30-110^{\circ} \mathrm{C}$ \\
\hline
\end{tabular}

Figure 5.16 shows the contribution of input pressure and temperature parameters on FPSO process and utility units. Ps and Ts represent the operating pressures and temperatures, and 1,2 and 3 are the numbers of each stage. The green, orange, red and black lines are liquid flow, gas flow, temperature, and pressure effects, respectively. The gray line presents the power demand. In that, the input parameters directly influence the separation train stages. The separated gas flow and liquid flow affect the separation train stages, VRU stages and MGC.The discharge pressure of MGC from operating pressures changes the power demand of MGC. Next, the separated volatile components as gas flow from separation stages form liquid in Hydrocarbon dew point control system (HDP) and the liquid flow is forwarded to the second stage of VRU. Then, after passing through heat exchanger and separator's VRU, the separated gas components are routed to MGC. In following, the remaining hydrocarbon liquids go to separation train. 
Finally, since the operating pressures are changed during the optimization procedure, the power consumption demand is altered according to the output pressures of the compressors and pumps, and to the changes in the gas/liquid mass flow rates sent to compressors/pumps. Thus, the amount of fuel required to met the total power demand is changed and, consequently, the exhaust gas temperature of the turbine changed and the temperature of the hot water supply to feed the heat exchangers of the separation train are also altered (Figure 5.16).

Figure 5.16 - Contribution of input parameters on FPSO process and utility units.

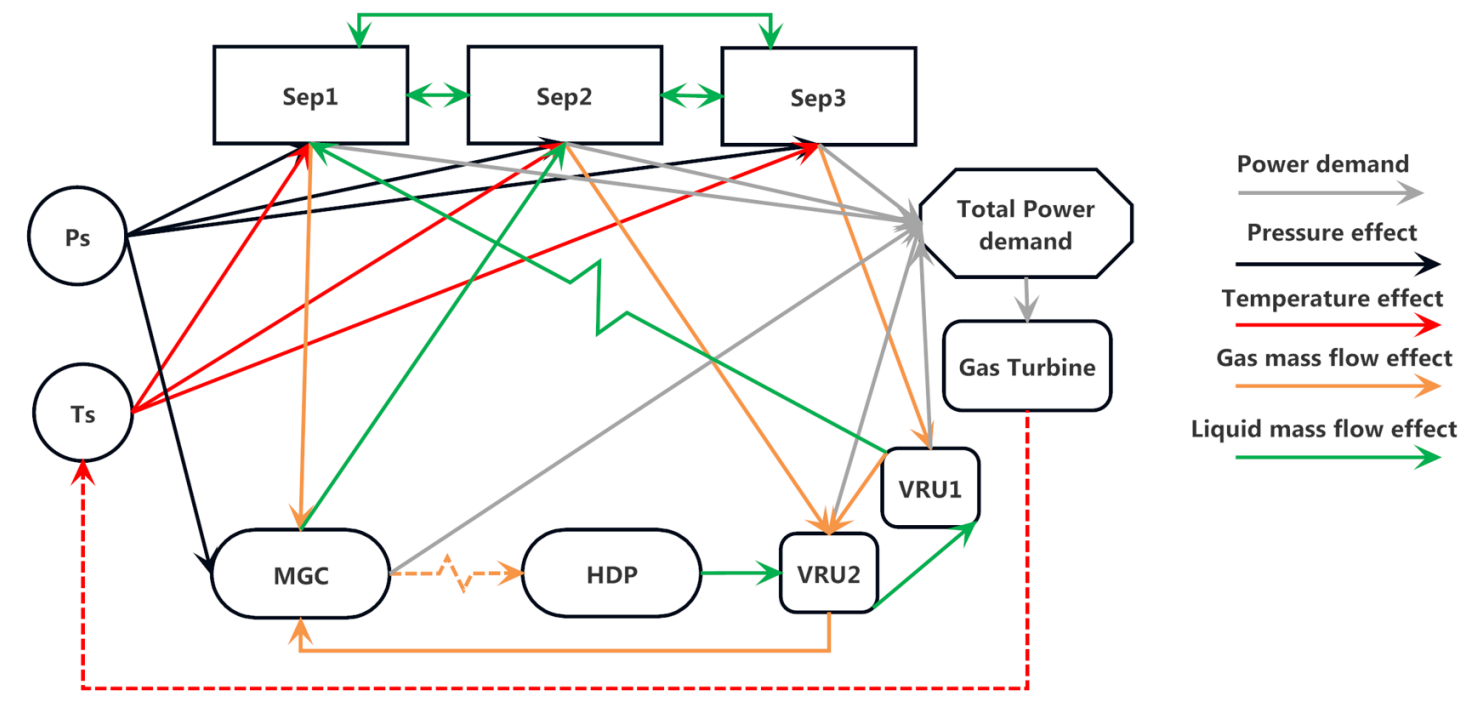

Source: Author

\subsection{Optimization Results}

This section is supposed to present the evaluation of the applied optimization methods and the results of objective functions from implemented optimization procedures.

\subsubsection{Assessment of applied optimization methods}

From literature review and problem conditions of the current thesis, the NSGA-II was chosen as an adequate optimization method for proposed modeled FPSO and objective functions. The initial effort for minimization of fuel consumption was performed and the results were analyzed. Then, it was decided to assess the other possible (and applicable) optimization algorithms regarding to experiences gained from the first execution. Therefore, four optimization methods from existing commercial codes were chosen to compete and compare with NSGA-Il's operation and results.

1) Firstly, one from gradient-based family optimization, AfilterSQP: This implementation of a Sequential Quadratic Programming algorithm uses an Adaptive Filter to ensure convergence. 
The adaptive filter helps achieve convergence starting from a random point in the search space. AfilterSQP formulates a quadratic programming (QP) problem, a line search is performed along the computed direction and the adaptive filter checks whether the point can be accepted. The adaptive filter helps achieve convergence starting from a random point in the search space. It considers the entire history of the current optimization run and accepts a new point only if it is not dominated by a previously computed point (Esteco SPA, 2017). Furthermore, because of the several numbers of accomplished works using the SQP methods in optimization of chemical and processing cycles, SQP was chosen. On the other hand, the current developed SQP can be implemented for both objective function and constraint satisfaction and it handles constraints by applying the Lagrange Multipliers methods. The maximization of hydrocarbon liquids of operating mode 1 as the objective function was defined and 8000 space designs were appointed as the maximum number of design evaluation.

As Figure 5.17 displays, after approximately 500 designs (with 200 designs of initial DoE), the optimization procedure is not converged and stopped. Therefore, it was found that SQP (or any modified one) is individually not suitable for this problem.

Figure 5.17 - SQP convergence curve for the function of hydrocarbon liquids recovery maximization.

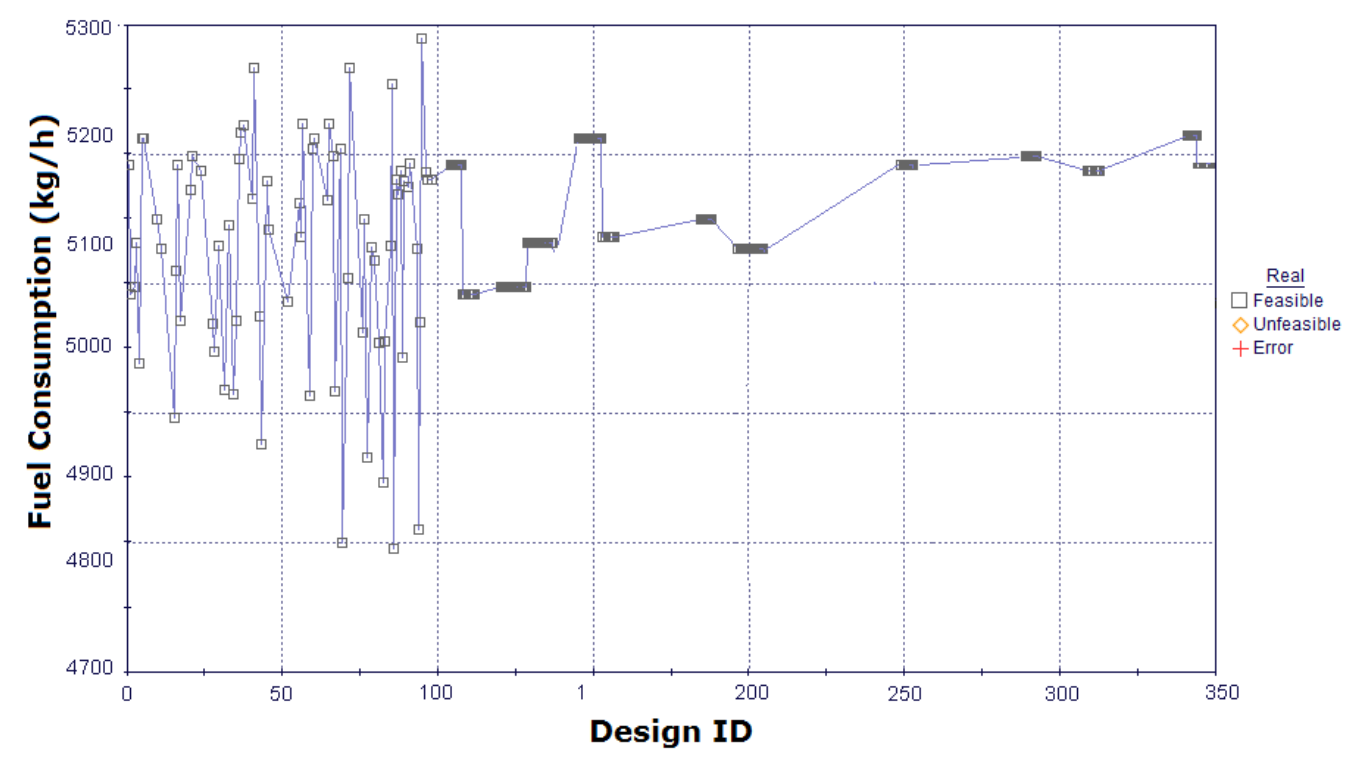

Source : Author

2) Simplex: The considered Simplex optimization algorithm implemented here is the Nelder-Mead or downhill simplex method. It does not compute derivatives, which makes it more robust than gradient-based methods and suitable for problems with noisy functions. This heuristic algorithm uses the concept of a simplex, which is a polyhedron with $N+1$ 
vertices in an $\mathrm{N}$-dimensional space ( $\mathrm{N}$ is the number of input variables). It compares the values of the objective function at $\mathrm{N}+1$ vertices and gradually moves the polyhedron towards the optimal point by iteratively replacing the worst vertex with a point moving through the centroid of the remaining $\mathrm{N}$ points. The algorithm stops either when it reaches the termination accuracy or the maximum number of evaluations (Esteco SPA, 2017). It is known as a very fast convergence and less required runs optimization method that may be useful compared to a very slow and numerous runs algorithms such as GA (SALVIANO et al., 2016).

Note that there is considered an Automatic Restart that optimization will restart each time the Termination Accuracy is reached and continue until the Maximum Number of Designs is generated. At each restart, the algorithm is initialized with the $\mathrm{N}+1 \mathrm{DoE}$ configurations that have not been used in the previous runs. If the DoE table contains fewer configurations than required for a run restart, the missing configurations are generated with a Random DoE.

Therefore, the fuel consumption minimization of operating mode 1 was selected as the objective function. Then, 8000 number of evaluations and five DoE designs were generated (four variables+1) as the initial sampling. As Figure 5.18 shows, however, the proposed Simplex stops after finding several optimum points by seven restarting and finally, only some of the local optimum points are shown as the solutions.

Figure 5.18 - Simplex convergence curve for the objective function of Fuel consumption minimization.

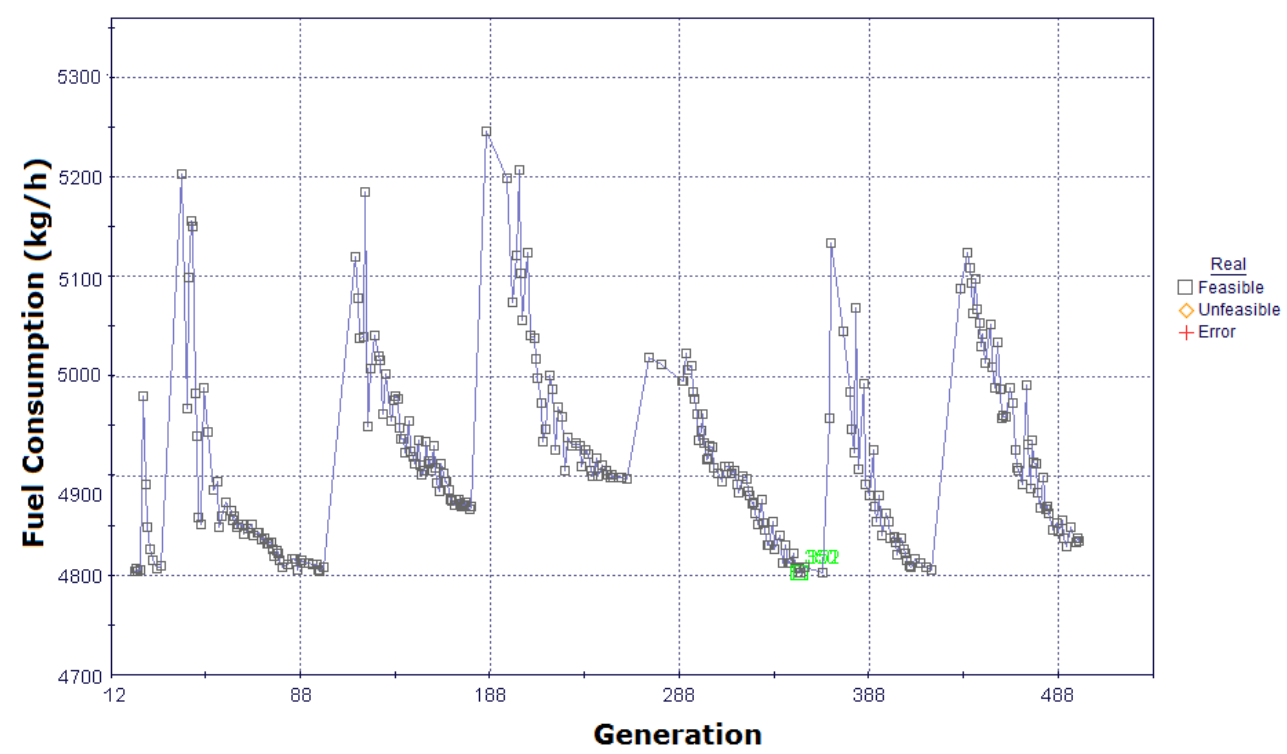

Source : Author

3) NSGA-II: Non-dominated sorting algorithms are in general computationally demanding, especially in case of very large populations, since the identification of individuals belonging 
to the first non-dominated front requires the comparison of each solution with every other solution. However, NSGA-II implements a smart non-dominated sorting strategy requiring much fewer computations. A domination count is computed for each solution: those with domination count 0 belong to the first front. Then, the domination count of all remaining dominated solution is reduced by 1 and those resulting with domination count 0 are classified to the second front (Esteco SPA, 2017). This procedure repeats until all designs became sorted. As shown in Figure 5.19, the NSGA-II explored several points to find the optimum point. However, after 8000 generations, NSGA-II dose not converged, but the highlighted points as local optimums are better (lower) than some points found by Simplex.

Figure 5.19-NSGA-II method convergence curve for the objective function of Fuel consumption minimization.

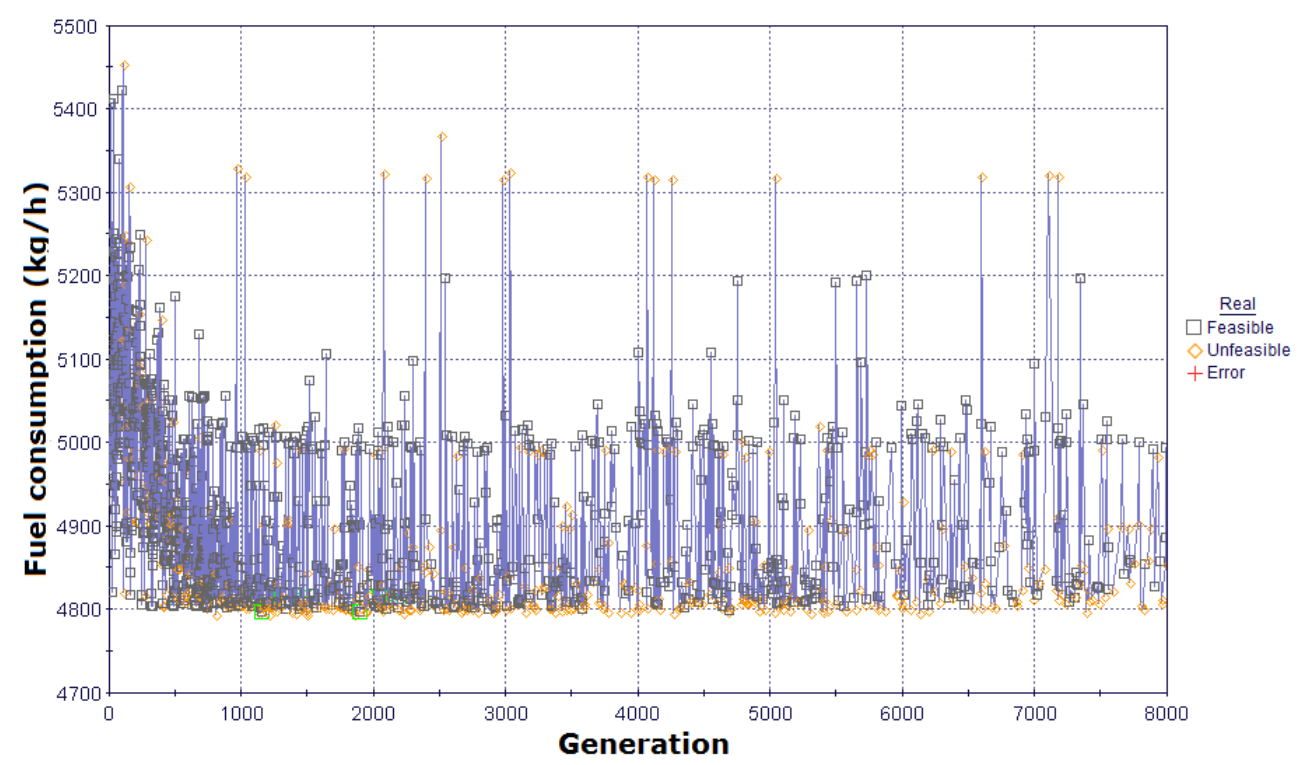

Source : Author

4) Hybrid method based on GA (NSGA-II+AFilterSQP): The SQP algorithm does not merely perform a local refinement around the quasi-optimal designs found by the genetic algorithm. On the contrary, the two algorithms cooperate and exchange information in several ways during the entire run. The genetic algorithm implemented in HYBRID promotes an efficient use of computational resources by combining the steady-state evolution with a controlled elitism procedure. The designs are ranked by applying the non-dominated sorting and crowding distance methods. The SQP algorithm is inserted in the run of the genetic algorithm as an extra operator, in addition to the classic GA operators (mutation and crossover). SQP runs therefore in parallel with the genetic algorithm. However, SQP is a sequential algorithm, so it performs only one evaluation at a time. All designs generated by SQP are added to those generated by the genetic algorithm and sorted and ranked at each 
periodic update. The SQP algorithm starts from a single randomly selected non-dominated design of the GA parent population, which thus enhances its search efficiency (Esteco SPA, 2017). Among all indicated algorithms above, the hybrid method had the fastest convergence (after simplex) and the best exploration points. Consequently, as Figure 5.20 shows, it is converged after about 2400 generations and the obtained result is $1 \%$ lower (better) than of NSGA-II.

Figure 5.20-Hybrid method convergence curve for the objective function of Fuel consumption minimization.

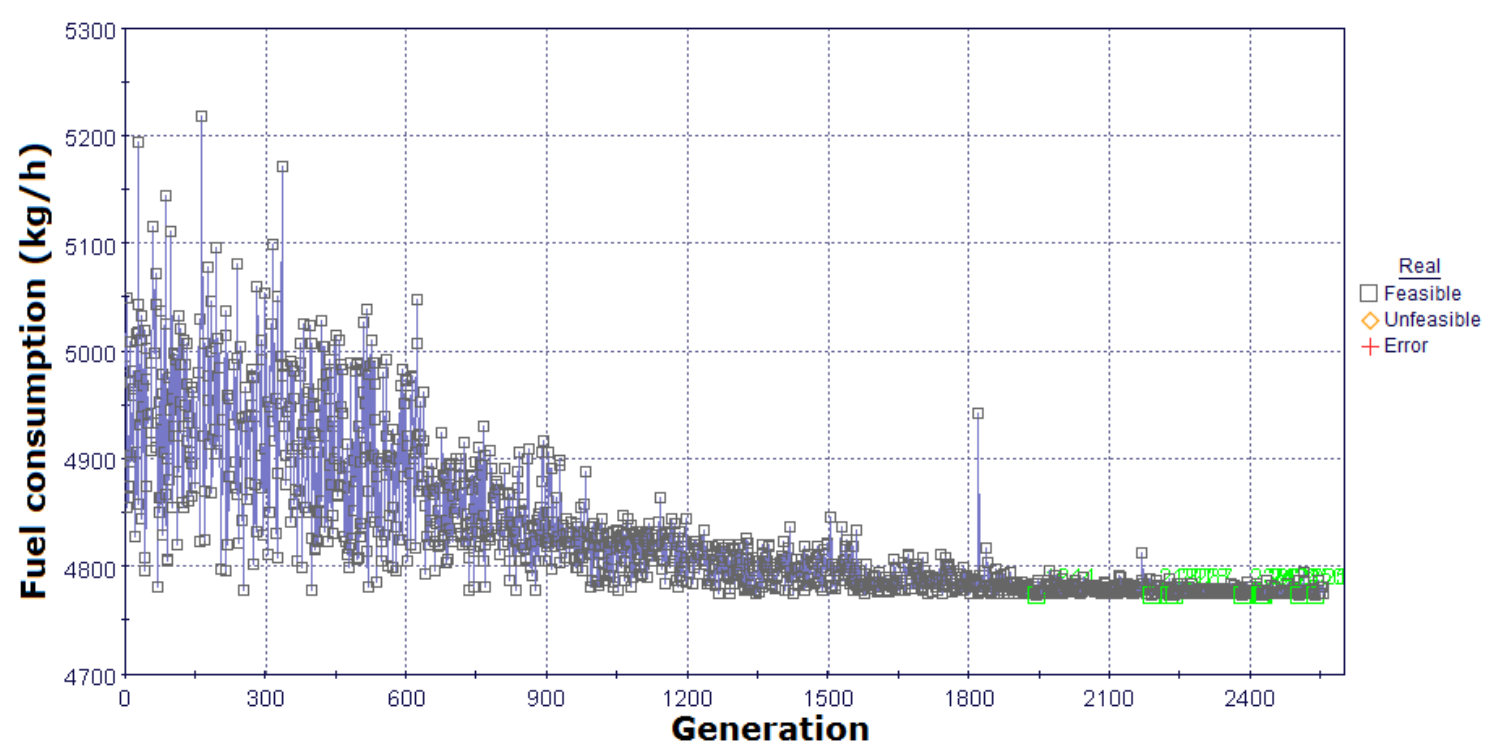

Source : Author

\subsubsection{Fuel consumption}

The current operating condition of a Brazilian FPSO, with its corresponding power consumption, fuel gas consumption and production, was taken as a baseline simulation (reference scenario) so that the optimization configuration could be compared. Both electrical and heating demands of FPSO were met by the gas turbine and by recovering the waste heat from the exhaust gas in the optimized case. However, the first and principal objective in this thesis and project was the minimization of fuel consumption in the optimization procedure, but, the exportation oil was also monitored, in order to avoid of its reduction. Moreover, the separation performance of separators during optimization process was analyzed.

\subsubsection{Operation Mode 1}

Table 5.8 shows the calculated fuel consumption and power demand of the FPSO unit for the baseline of operation mode 1 and optimized case. The optimization procedure using 
the Hybrid method and after 2500 generations was converged on the feasible minimum ( Appendix D, Figure D.1). The initial population was generated for 200 designs and maximum evaluation designs of optimization method were defined 8000 generations. The optimization process results was compared with computed operating data of the baseline. As can be seen in Table 5.8, the optimization procedure of fuel minimization found an operating configuration with a mitigation of $6.4 \%$ in the required power of the plant with an associated reduction of $4.46 \%$ in fuel consumption. Moreover, the exportation oil increased about $1.9 \%$ when compared to the baseline case. Due to an improvement in oil recovery and stabilization conditions, the mass flow of gas production was decreased, which reduced the required power of the compression systems. Since the compressors were the greatest consumers of power in main processing plants of an FPSO (Figure 5.1), the optimized case presented the indicated reduction of fuel consumption and an increase in exportation oil.

Table 5.8 - Fuel consumption and power demand of the FPSO unit for the baseline and optimized case of operation mode 1

\begin{tabular}{cccc|cc|cc}
\hline & & $\begin{array}{c}\text { FUE } \\
(\mathrm{kg} / \mathrm{h})\end{array}$ & $\%$ & $\begin{array}{c}\text { Exp. oil } \\
(\mathrm{kg} / \mathrm{h})\end{array}$ & $\%$ & $\begin{array}{c}\text { Power Cons. } \\
(\mathrm{MW})\end{array}$ & $\%$ \\
\hline \multirow{2}{*}{ Mode 1 } & Baseline case & 4997 & & 442127 & & 26.4 & \\
& Optimized case & 4774 & & 450546 & & 24.7 & \\
\hline
\end{tabular}

Table 5.9 shows the input parameters for the baseline and optimized configurations. The input pressure of the first pressure valve is $2300 \mathrm{kPa}$ and its output pressure (P1) is 1300 $\mathrm{kPa}$ and $1284 \mathrm{kPa}$ for the baseline case and optimized case, respectively. The value of P1, as explained before in sensitivity analysis, is important to decreases the shaft work of the main gas compressor. In addition, the value P1 found by the optimization procedure is also due to the maximization of the separation of light hydrocarbons at the medium pressure level and to the better stabilization of separated intermediate hydrocarbons in the liquid. Thus, smaller shaft power of the compressors is required, since the smaller mass flow of the gas stream follows to the compressors to recover the pressure of the gas stream to initial feed pressure of MGC, resulting in a reduction in the total power demand.

The optimum operating condition of the end of the second stage of separation (P2) is important for stabilizing the separated liquid flow in the second stages. The optimization method generated the value of $915 \mathrm{kPa}$ for P2. Regarding parameter P3, which is the pressure of the third stage of the separation train, the value found by the optimization algorithm improved the separation of the intermediate and heavy hydrocarbons in the third stage.

Moreover, as the output pressure of the separated gas flow in the third stage of separation train should be recovered by the compressor of the vapour recovery unit to the initial feed 
pressure of the main compressor, any changes in P3 could increase the shaft work of the compressors. The optimization procedure found $\mathrm{P} 3=448 \mathrm{kPa}$ for operation mode 1 ; in turn, the values for the baseline configuration are P3 $=244 \mathrm{kPa}$ for operation mode 1 . Thus, the compressors power demand is smaller for the optimized case and, as consequence, a decrease of fuel consumption for the optimized case is observed and it proves the results of sensitivity analysis for P3 as the major effect. Finally, for inlet parameter of Pc, when it increases, a higher compressor shaft work is required to achieve the necessary output pressure. For this reason, the optimization algorithm found $\mathrm{Pc}=7019 \mathrm{kPa}$ for operation modes1, i.e., the minimum feasible value in order to keep the stability of the whole plant (especially to guarantee the expected oil production).

Figure 5.21 shows the separated gas flow composition in the third stage of separation train for the baseline and optimized cases for operation mode 1

Table 5.9 - Input parameters for the baseline and optimized configurations of operation mode 1.

\begin{tabular}{cccccc}
\hline & & $\begin{array}{c}\text { P1 } \\
(\mathrm{kPa})\end{array}$ & $\begin{array}{c}\mathrm{P} 2 \\
(\mathrm{kPa})\end{array}$ & $\begin{array}{c}\mathrm{P} 3 \\
(\mathrm{kPa})\end{array}$ & $\begin{array}{c}\text { Pc } \\
(\mathrm{kPa})\end{array}$ \\
\hline \multirow{2}{*}{ Mode 1 } & Baseline case & 1300 & 440 & 244 & 8196 \\
& & & & & \\
& Optimized case & 1284 & 915 & 448 & 7019 \\
\hline
\end{tabular}

Figure 5.21 - The separated gas flow composition in the third stage of separation train for the baseline and optimized cases for operation mode 1.

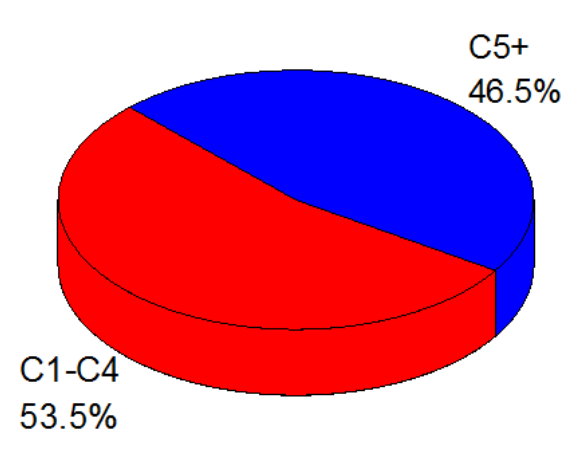

Base line

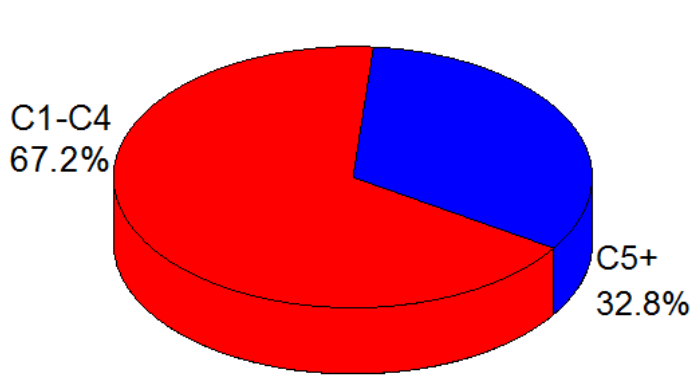

Optimized

Source : Author

As observed in Figure 5.21, the separation of the associated natural gas components in 
the third stage for operation mode 1 consists of $53.5 \%$ of $\mathrm{C} 1-\mathrm{C} 4$ and $46.5 \%$ of $\mathrm{C} 5+$ for the baseline versus $67.2 \%$ of $\mathrm{C} 1-\mathrm{C} 4$ and $32.8 \%$ of $\mathrm{C} 5+$ for the optimized case. Consequently, this improvement of separated volatile components (in liquid form) in the third stage of separation leads an increase in the total mass flow of liquid production of the processing plant. However, this obtained value is not significant, but its improvement, considerably affects the total power demand.

\subsubsection{Operation mode 2}

Table 5.10 shows the calculated fuel consumption and power demand of the FPSO unit for the baseline and optimized cases. The DoE designs of this optimization algorithm were 150 designs and 8000 evaluations were considered as maximum generations of the optimization process. The convergence of the optimum value of the fuel consumption for operation mode 2 was obtained after approximately 1200 generations (Appendix D, Figure D.2). Table 5.10 allows observing that the optimized case for operation modes 2 leads to a mitigation of $8.34 \%$ on fuel consumption of the FPSO unit, in comparison to the baseline. This decrease in fuel consumption is due to the reduction of the power demand of the whole plant observed in the optimized case (Table 5.10). For operation mode 2, the comparison between optimized and baseline cases reveals a reduction of $10 \%$ in power demand and an increase of $3.78 \%$ in exportation oil.

Table 5.10 - Fuel consumption and power demand of the FPSO unit for the baseline and optimized case of operation mode 2.

\begin{tabular}{lccc|cc|cc}
\hline & & $\begin{array}{c}\text { FUE } \\
(\mathrm{kg} / \mathrm{h})\end{array}$ & $\%$ & $\begin{array}{c}\text { Exp. oil } \\
(\mathrm{kg} / \mathrm{h})\end{array}$ & $\%$ & $\begin{array}{c}\text { Power Con. } \\
(\mathrm{MW})\end{array}$ & $\%$ \\
\hline \multirow{2}{*}{ Mode 2 } & Baseline case & 3139 & & 334517 & & 14.38 & \\
& & & -8.34 & & +3.78 & & -10 \\
\hline
\end{tabular}

The values of $\mathrm{P} 3, \mathrm{Pc}$ and $\mathrm{T} 2$ for the baseline case and found by the optimization procedure for operation modes 2 are depicted in Table 5.11. As explained before for operation mode 1 , the value found of parameter P3 (which is the operating pressure of the third stage of the separation train) by the optimization algorithm affects directly the separation of the hydrocarbons in the third stage.

Thus, as Figure 5.22 shows, the separation of the associated natural gas components in the third stage for operation mode 2 consists of $54.5 \%$ of $\mathrm{C} 1-\mathrm{C} 4$ and $45.5 \%$ of $\mathrm{C} 5+$ for the baseline versus $89.1 \%$ of $\mathrm{C} 1-\mathrm{C} 4$ and $10.9 \%$ of $\mathrm{C} 5+$ for the optimized case.

The effect of T2 is due to the separation of the intermediate hydrocarbons, such as propane and butanes in the gas flow, and heavy components of intermediate hydrocarbons, such as pentane in the liquid phase. In addition, the shaft power of the oil pump can be influenced 
Table 5.11 - Input parameters for the baseline and optimized configurations of operation mode 2

\begin{tabular}{ccccc}
\hline & & $\begin{array}{c}\mathrm{P} 3 \\
(\mathrm{kPa})\end{array}$ & $\begin{array}{c}\mathrm{Pc} \\
(\mathrm{kPa})\end{array}$ & $\begin{array}{c}\mathrm{T} 2 \\
\left({ }^{\circ} \mathrm{C}\right)\end{array}$ \\
\hline \multirow{2}{*}{ Mode 2 } & Baseline case & 232 & 7944 & 88 \\
& & & & \\
& Optimized case & 449 & 7020 & 37 \\
\hline
\end{tabular}

by the operating temperature in the optimized case, which is lower than the temperature of the baseline configuration, as shown in Table 5.11. The optimal combination of T2 and P3 stabilizes the volatile components in the oil stream leading to lower compressor shaft power. Hence, the optimization procedure has found a maximum separation of light hydrocarbons in the gas flow and the maximum recovery of heavy hydrocarbons in oil stream, improving the stabilization of the volatile components leaving the last stage of separation.

Figure 5.22 - The separated gas flow composition in the third stage of separation train for the baseline and optimized case for operation mode 2 .

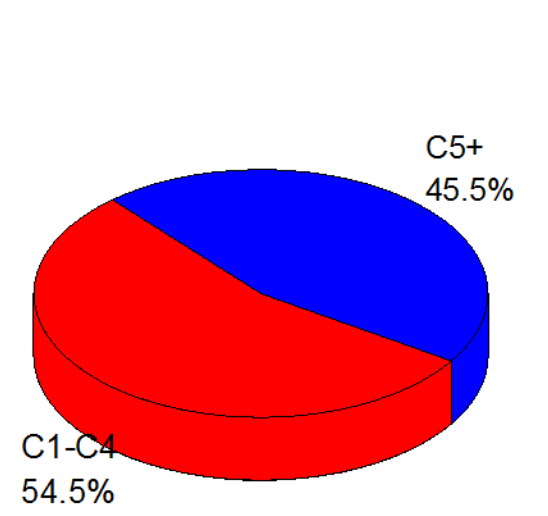

Base line

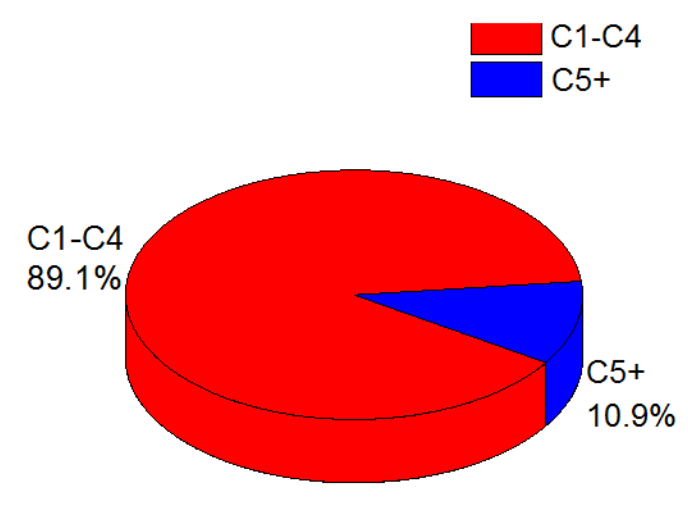

Optimized

Source : Author

As the output pressure of the separated gas flow in the third stage of separation train should be recovered by the compressor of the vapour recovery unit to the initial feed pressure of the main compressor, any changes in $\mathrm{P} 3$ could increase the shaft work of the compressors. The optimization procedure found $\mathrm{P} 3=449 \mathrm{kPa}$ for operation mode 2; in turn, the values for the baseline configuration are $\mathrm{P} 3=232 \mathrm{kPa}$ for operation mode 2 . Thus, the compressors power demand is smaller for the optimized case and, as consequence, a decrease of fuel consumption for the optimized case is observed. In term of the power consumption of the 
main compressors and inlet parameter Pc, when it increases, a higher compressor shaft work is required to achieve the necessary output pressure. For this reason, the optimization algorithm found $\mathrm{Pc}=7020 \mathrm{kPa}$, considering the minimum feasible value in order to keep the stability of the all processing plant that is check by the process simulator.

\subsubsection{Operation mode 3}

Table 5.12 shows the calculated fuel consumption and also power demand of the FPSO unit for the baseline and optimized cases of operation mode 3 . In that, there is a mitigation of $2.43 \%$ on the FPSO units fuel consumption, in comparison to the baseline. It is also observed in Table 5.12 that the comparison between optimized and baseline cases reveals a reduction of $2.9 \%$ in power demand for operation mode 3 . In addition, an increase of $1.7 \%$ in exportation oil mass flow of optimized case is reported compared to the baseline case. The DoE designs of this optimization algorithm were 150 designs and 8000 evaluations were considered as maximum generations of the optimization process. The convergence of the optimum value of fuel consumption for operation mode 3 was obtained after approximately 3000 generations (Appendix D, Figure D.3).

Table 5.12 - Fuel consumption and power demand of the FPSO unit for the baseline and optimized case of operation mode 3.

\begin{tabular}{lccc|cc|cc}
\hline & & $\begin{array}{c}\text { FUE } \\
(\mathrm{kg} / \mathrm{h})\end{array}$ & $\%$ & $\begin{array}{c}\text { Exp. oil } \\
(\mathrm{kg} / \mathrm{h})\end{array}$ & $\%$ & $\begin{array}{c}\text { Power Con. } \\
(\mathrm{MW})\end{array}$ & $\%$ \\
\hline \multirow{2}{*}{ Mode3 3} & Baseline case & 3201 & & 148610 & & 14.72 & \\
& Optimized case & 3123 & & 151138 & & 14.29 & \\
\hline
\end{tabular}

The values of input parameters for the baseline and optimized configurations of operation mode 3 is shown in Table 5.13. There, except the operating temperature of the second stage of separation train (T2), the value of optimization procedure for other parameters are almost the same with operation mode 2 . It is because of very close hydrocarbons composition among operation mode 2 and operation mode 3 .

According to McCain et al. (2011) and the explanations in previous chapters, the compositions of operation modes 2 and 3 are included in the wet gas group. Wet gas is a special two-phase flow (gas-liquid) with volatile components which is often encountered in condensate compositions. Thus, the optimal combination of T2 and P3 stabilizes the volatile components in the oil stream leading to lower compressor shaft power. Hence, the optimization procedure has found a maximum separation of light hydrocarbons in the gas flow and the maximum recovery of heavy hydrocarbons in oil stream, improving the stabilization of the volatile components leaving the last stage of separation. However, the volatile components of wet gas compared to 
condensate oil is fewer and this behavior is used in hydrocarbon liquids recovery subsection to maximize the exportation oil.

Table 5.13 - Input parameters for the baseline and optimized configurations of operation mode 3

\begin{tabular}{ccccc}
\hline & & $\begin{array}{c}\mathrm{P} 3 \\
(\mathrm{kPa})\end{array}$ & $\begin{array}{c}\mathrm{Pc} \\
(\mathrm{kPa})\end{array}$ & $\begin{array}{c}\mathrm{T} 2 \\
\left({ }^{\circ} \mathrm{C}\right)\end{array}$ \\
\hline \multirow{2}{*}{ Mode 3 } & Baseline case & 229 & 7287 & 88 \\
& & & & \\
& Optimized case & 447 & 7020 & 48 \\
\hline
\end{tabular}

In operation mode 3 , the separation of the associated natural gas in the third stage is $64.2 \%$ of $\mathrm{C} 1-\mathrm{C} 4$ and $35.8 \%$ of $\mathrm{C} 5+$ for the baseline versus $87.5 \%$ of $\mathrm{C} 1-\mathrm{C} 4$ and $12.5 \%$ of $\mathrm{C} 5+$ of the optimized case (Figure 5.23).

Figure 5.23 - The separated gas flow composition in the third stage of separation train for the baseline and optimized cases for operation mode 3 .

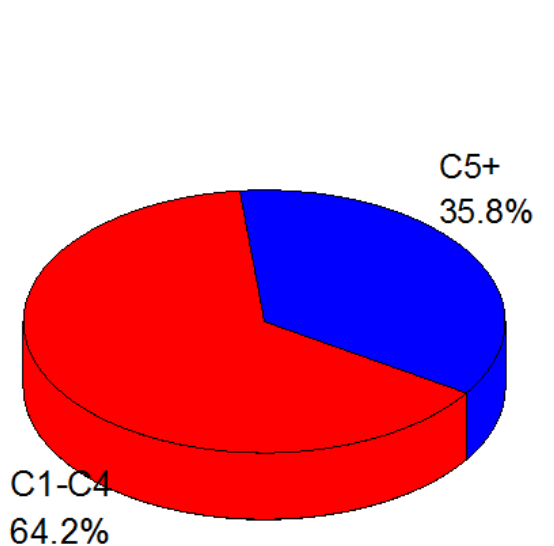

Base line

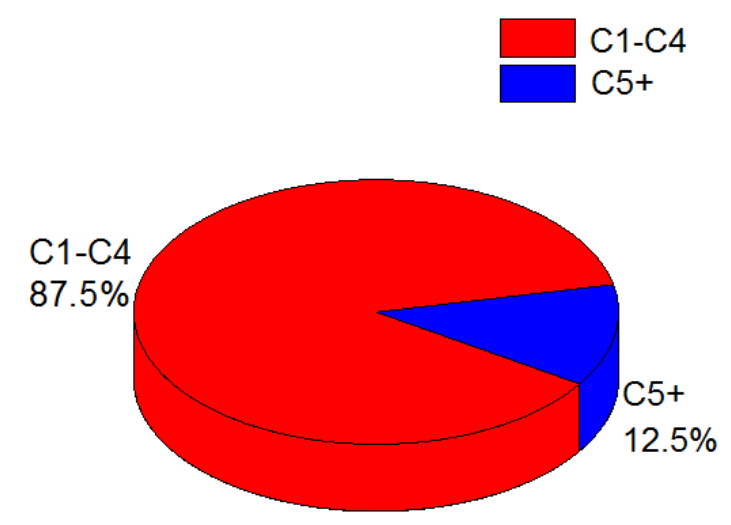

Optimized

Source : Author

After all, the oil production rate as hydrocarbon liquids recovery is not considered as a direct objective function for the optimization process. However, the operating pressures and the operating temperatures, of the FPSO unit are changed to search the minimum fuel consumption, the recovery of hydrocarbon liquid components in separation processes changes as well. Note that the crude oil composition evaluated in this research has volatile components with condensate molecules. Thus, since the crude oil stabilization is also influenced by pressure and temperature operating conditions, a smaller value of gas mass 
flow is required to be compressed by vapor recovery compressors and by the main gas compressor. Thereupon, the maximization of Hydrocarbon liquids recovery will be considered as a direct objective in the next subsection to assess its results by separation performance indicators.

\subsubsection{Hydrocarbon liquids recovery}

From point of view of Abdel-Aal et al. (2015), for a separation process, the aim of oil production facilities in terms of separation performance are : (1) separating the light hydrocarbons components ( $\mathrm{C} 1$ and $\mathrm{C} 2$ ) from oil, (2) maximizing the recovery of the intermediate hydrocarbons (C3, C4, and C5) in the oil product, and (3) saving heavy components (C6+) in the oil product. However, the light components contained in liquid production (Oil or/and condensate) flash to gas in the storage tank, because of processing issues, when the pressure within the export tanks or pipelines will be reduced. On the other hand, intermediate components, which are the major substances of a crude oil, are released by undergoing a pressure drop of separators.

Therefore, one of the most important design aspect of an oil and gas processing offshore facility is the performance of separating the light and intermediate components into gas and oil products to achieve the maximum oil recovery (KIM et al., 2014). Hence, these important design parameters together are named hydrocarbon liquids recovery (and stabilization) and are selected as an objective function of an optimization procedure for three operation modes.

In order to analyze the behavior of hydrocarbons components for three operation modes, some points in separation train (as the responsible of separation processes) are selected. Figure 5.24 shows the selected process points to investigate the phase stabilization (gas or liquid) in the optimization procedure regarding p0, p1, p2, p3, p4, p5, p6, and p7. Point 0 represents the input condition before the first pressure valve and it is important to show the initial value of the oil mass flow and associated gas flow.

- Output of the first control valve (p1);

- Output of the first heat exchanger (p2);

- Output of the second heat exchanger (p3);

- Input of pair separator and after mixing with recycle liquid content of MGC (p4);

- Output of the first separator of the second stage of the separation train (p5);

- Output of second stage of separation train and before mixing with dilution water (p6) and

- Input of the third stage of the separation train (p7).

Note that the symbol of input variables are with $\mathbf{P}$ (uppercase) and process points are with $\mathbf{p}$ (lowercase). 
Figure 5.24 - Selected point in process steps of separation.

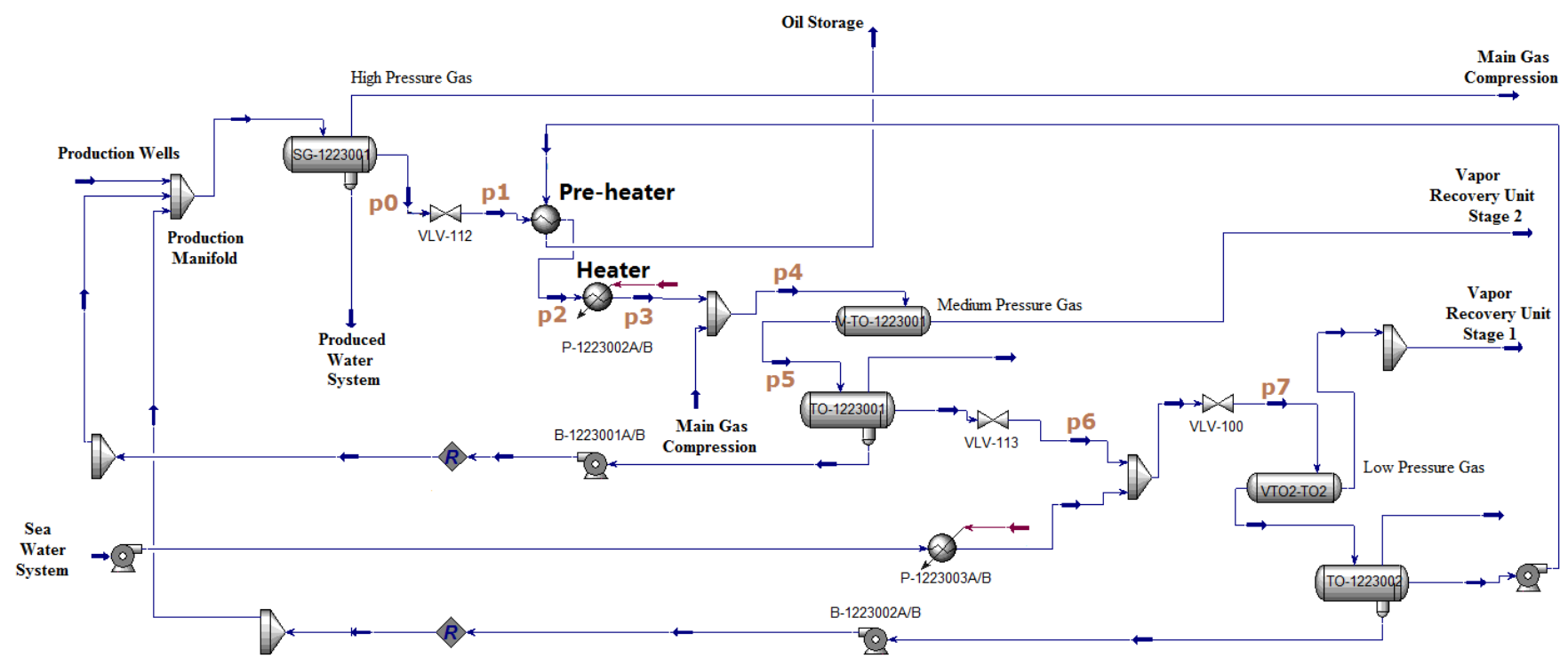

Source : Author

\subsubsection{Operation mode 1}

In this subsection, the optimized configurations of operation mode 1 to achieve the maximum hydrocarbon liquids recovery was discussed and compared with the baseline configuration. As the crude oil composition of this operation mode was condensate oil, the recovery of volatile components and stabilization and saving oil components have attracted more attention. The finding optimum procedure was performed using $150 \mathrm{DoE}$ designs (ULH and ISF) and a maximum number of evaluation was settled for 8000 designs. The optimization convergence was achieved after 3000 generations (Appendix D, Figure D.4) in the value of $462300(\mathrm{~kg} / \mathrm{h})$ for exportation oil.

Table 5.14 shows the input parameters for the baseline and optimized configurations. The input pressure of the first pressure valve is $2300 \mathrm{kPa}$ and its output pressure (P1) is $1300 \mathrm{kPa}$ and $1302 \mathrm{kPa}$ for the baseline case and optimized case, respectively. Next, the optimization procedure found P2 $=560 \mathrm{kPa}$ and P3 $=449 \mathrm{kPa}$ for optimization case while the values for the baseline configuration are P2 $=440 \mathrm{kPa}$ and P3 $=244 \mathrm{kPa}$ for operation mode 1 . The significant difference among baseline case and optimization is reported for T2 that the temperature of petroleum at the heater for the baseline case is $90^{\circ} \mathrm{C}$, while for the optimized case, it is $37.3^{\circ} \mathrm{C}$ (Table 5.14). The justification of this results is expressed in Figures 5.25a $\& 5.25 b$.

Figures $5.25 \mathrm{a} \& 5.25 \mathrm{~b}$ show the stabilization of phase during the separation processes for the baseline and optimized case of operation mode 1 regarding presented points in 
Table 5.14 - Input parameters for the baseline and optimized configurations of operation mode 1 - Hydrocarbon liquids recovery case.

\begin{tabular}{cccccc}
\hline & & $\begin{array}{c}\mathrm{P} 1 \\
(\mathrm{kPa})\end{array}$ & $\begin{array}{c}\mathrm{P} 2 \\
(\mathrm{kPa})\end{array}$ & $\begin{array}{c}\mathrm{P} 3 \\
(\mathrm{kPa})\end{array}$ & $\begin{array}{c}\mathrm{T} 2 \\
\left({ }^{\circ} \mathrm{C}\right)\end{array}$ \\
\hline \multirow{2}{*}{ Mode 1 } & Baseline case & 1300 & 440 & 244 & 90 \\
& & & & & \\
& Optimized case & 1302 & 560 & 449 & 37.3 \\
\hline
\end{tabular}

Figure 5.24. The black line shows the gas phase components flow rate while the blue line represents the liquid phase components flow rate. Up to point 3 , the total gas mass flow rate is separated by the first stage of the separator( including pre-heater and heater units). The effect of T2 is due to the separation of the intermediate hydrocarbons, such as propane and butanes in the gas flow, and heavy components of intermediate hydrocarbons, such as pentane in the liquid phase. The change of mass flow (both gas and liquid) for baseline case and optimization between p2 and p3 is because of T2. Therefore, this argument proves the results of sensitivity analysis of T2 for hydrocarbons liquid recovery in operation mode 1. Next, due to the very small rate of recycling pipe from MGC, the conditions of $p 3$ and p4 are the same for oil (Figures 5.25a \& 5.25b). Then, all of the gas is separated after point 4 as a medium pressure gas and, consequently, the gas mass flow rate is zero at point 5 . At p6 and p7(after pressure valves VLV-113 and VLV-100), the gas flow content is separated from liquid content.

Moreover, as shown in Figures 5.25a \& 5.25b, from the same composition of the crude oil, an increase of the oil recovery and stabilization for the optimized case are verified when compared to the baseline. The optimum operating condition led to an improvement of the separation performance of the gas and liquid hydrocarbons. As the crude oil stream is involved by dissolved gas components, the heavy components of the intermediate hydrocarbons, condensate components and pseudo-component of petroleum, the variation in pressure and temperature influences the phase stabilization of these components.

For example, as shown in Figures 5.25a \& 5.25b, at point 3, the optimum configuration provides a condition to maximize the stabilization of intermediate hydrocarbons in the liquid phase, especially propane and butane (466400 kg/h). In turn, for the baseline, a large amount of heavy components of intermediate hydrocarbons is separated in the gas phase (17000 kg/h). Moreover, at point 7, the exportation of oil increased about $20173 \mathrm{~kg} / \mathrm{h}$ by the improvement in the recovery of oil components at the optimum configuration (Figures 5.25a $\& 5.25 b)$.

Table 5.15 shows the exportation oil rate, separation performance of all separators and propane, butane and pentane percentage in exportation oil of the FPSO unit for the baseline and optimized case of operation mode 1 . The optimization process found an 
operating configuration with an increase of $4.36 \%$ of exportation oil as compared with baseline configuration. Consequently, The results from the optimized case indicated that the maximization of hydrocarbons liquid recovery is achieved by increasing the operating pressure in the second and the third stages of the separation train and by decreasing the operating temperature in oil heater of the separation train for operation mode1.

In the optimization process, exportation oil rate is considered as the objective function and two indicators are monitored. Thus, the separation performance of separators (all three stages) from equation 3.6 is calculated and the content of intermediate hydrocarbon components ( $\mathrm{C} 3, \mathrm{C} 4$ and $\mathrm{C} 5)$ in exportation oil is used to analyze the quality of exportation oil. Next, two indicators (one from (KIM et al., 2014) and the other one from (NGUYEN; OLIVEIRA-JR, 2016g)) are selected to analyze the optimization of hydrocarbon liquids recovery. For example, in equation 3.6, C1-C4 should be separated in gas flow while in (KIM et al., 2014) cited from (ABDEL-AAL et al., 2015) is indicated that C3 and C4 also should be separated in oil product.

Table 5.15 shows that the separation performance of the optimization case is increased from $65 \%$ of baseline to $68 \%$ and the C3-C5 content of optimization case has an increase of $10 \%$ as compared with baseline case. Moreover, as observed in Table 5.15, the power demand of optimization case is decreased by $4.9 \%$ when compared to the baseline case due to less compressed of gas mass flow by compressors (MGC and EGC) . 
Figure 5.25 - Stabilization of phase during the separation processes for the baseline and optimized cases of operation mode 1.

(a)

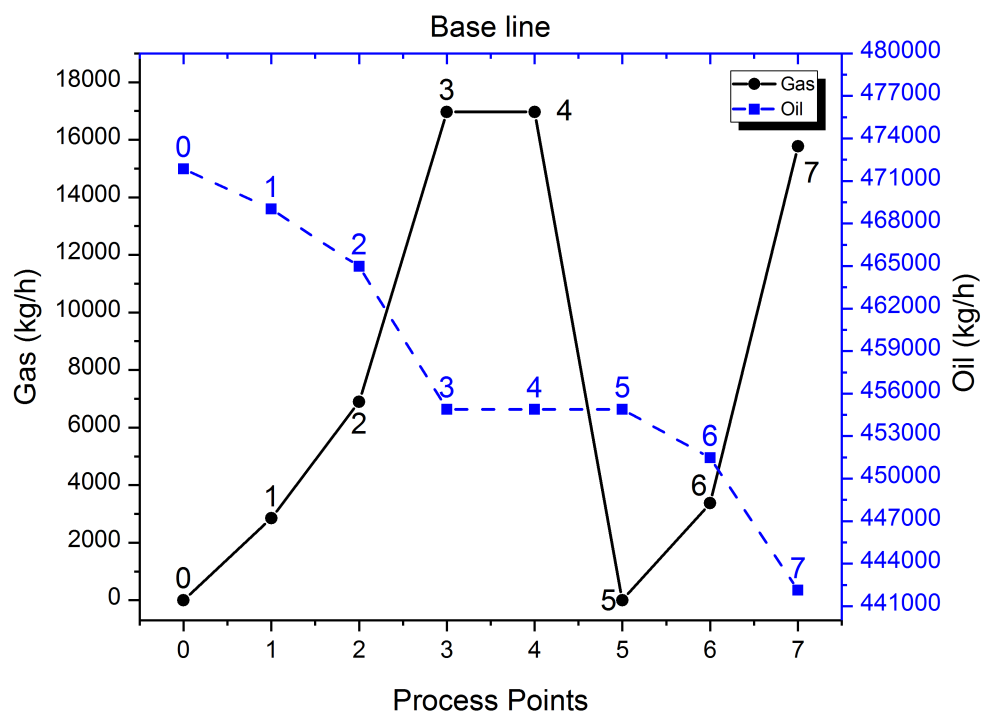

(b)

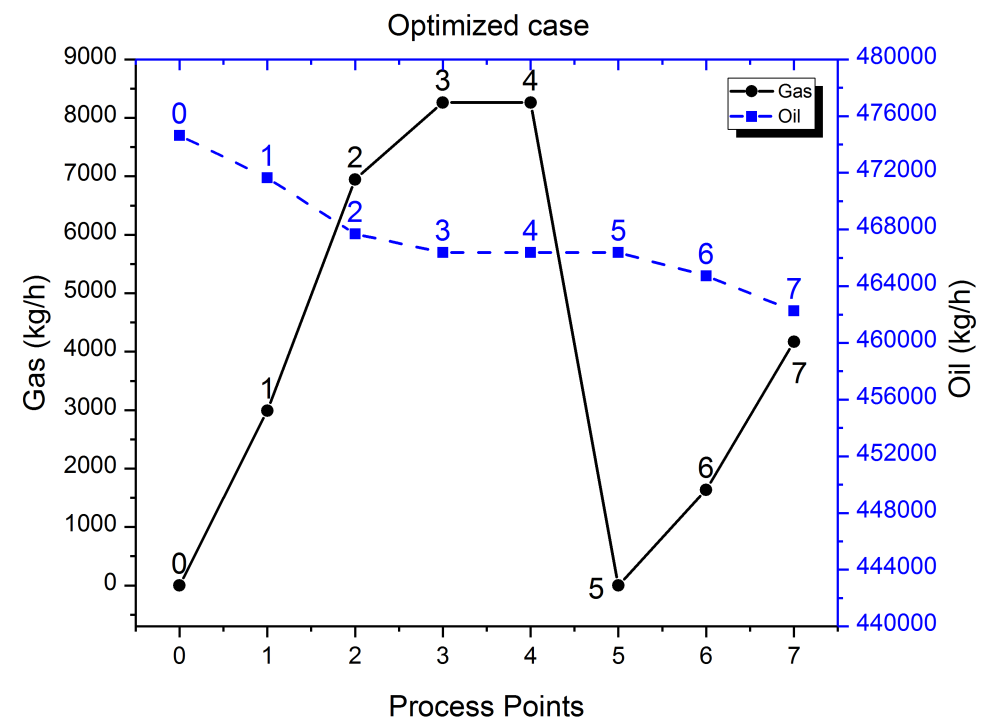

Source : Author 
Table 5.15 - Exportation oil rate, separation performance of all separators and propane, butane and pentane percentage of exportation oil of the FPSO unit for the baseline and optimized case of operation mode 1.

\begin{tabular}{cccc|c|c|cr}
\hline & & $\begin{array}{c}\text { Exp. oil } \\
(\mathrm{kg} / \mathrm{h})\end{array}$ & $\begin{array}{c}\text { Sep. Per. } \\
\%\end{array}$ & $\begin{array}{c}\text { C3-C5 } \\
\%\end{array}$ & $\begin{array}{c}\text { Power Con. } \\
(\mathrm{MW})\end{array}$ & $\%$ \\
\hline \multirow{2}{*}{ Mode 1 } & Baseline case & 442127 & & 65 & 6 & 26.4 & \\
& Optimized case & 462300 & +4.36 & 68 & 16 & 25.1 & \\
\hline
\end{tabular}

\subsubsection{Operation mode 2}

Table 5.16 shows the input parameters for the baseline and optimized configurations of operation mode 2 with hydrocarbon liquids recovery as the objective function. The DoE designs of this optimization algorithm for the initial population are 150 designs and 8000 evaluations are considered as maximum generations of the optimization algorithm. The convergence of the optimum value of hydrocarbon liquids recovery for operation mode 2 is obtained after approximately 3000 generations (Appendix D, Figure D.5 ). The optimization procedure found $\mathrm{T} 2=35{ }^{\circ} \mathrm{C}$ for operation mode 2 ; in turn, the values for the baseline configuration is $\mathrm{T} 2=88^{\circ} \mathrm{C}$. The effect of Tc on separation of hydrocarbon components is shown in Figures 5.26a \& 5.26b between p2 and p3. Figures 5.26a \& 5.26b show the stabilization of phase during the separation processes for the baseline and optimized case of operation mode 2 . The black line shows the gas phase components rate while the blue line represents the liquid phase components rate. In that, the stabilization of intermediate hydrocarbons increases, so that the mass flow rate of oil in p3 of the optimized case is about $6150 \mathrm{~kg} / \mathrm{h}$ more than p3 of the baseline case.

Table 5.16 also shows $\mathrm{P} 2=752 \mathrm{kPa}$ and $\mathrm{P} 3=450$ of optimization case for operation mode 2 versus, the values for the baseline configuration are $\mathrm{P} 2=428 \mathrm{kPa}$ and $\mathrm{P} 3=232 \mathrm{kPa}$ for operation mode 2. Figures 5.26a \& 5.26b prove the optimization results of P2 and P3 that oil saving and recovery increases in the end of the second stage and third stage of separation train (between $\mathrm{p} 5$ to p7). Consequently, the results from the optimized case indicated that the maximization of hydrocarbons liquid recovery is achieved by increasing the operating pressure in the second and the third stages of the separation train and by decreasing the operating temperature in oil heater of the separation train for operation mode 2. 
Table 5.16 - Input parameters for the baseline and optimized configurations of operation mode 2 - hydrocarbon liquids recovery case.

\begin{tabular}{ccccc}
\hline & & $\mathrm{P} 2$ & $\mathrm{P} 3$ & $\mathrm{~T} 2$ \\
$(\mathrm{kPa})$ & $(\mathrm{kPa})$ & $\left({ }^{\circ} \mathrm{C}\right)$ \\
\hline \multirow{2}{*}{ Mode 2 } & Baseline case & 428 & 232 & 88 \\
& & & & \\
& Optimized case & 752 & 450 & 35 \\
\hline
\end{tabular}

Figure 5.26 - Stabilization of phase during the separation processes for the baseline and optimized case of operation mode 2 .

(a)

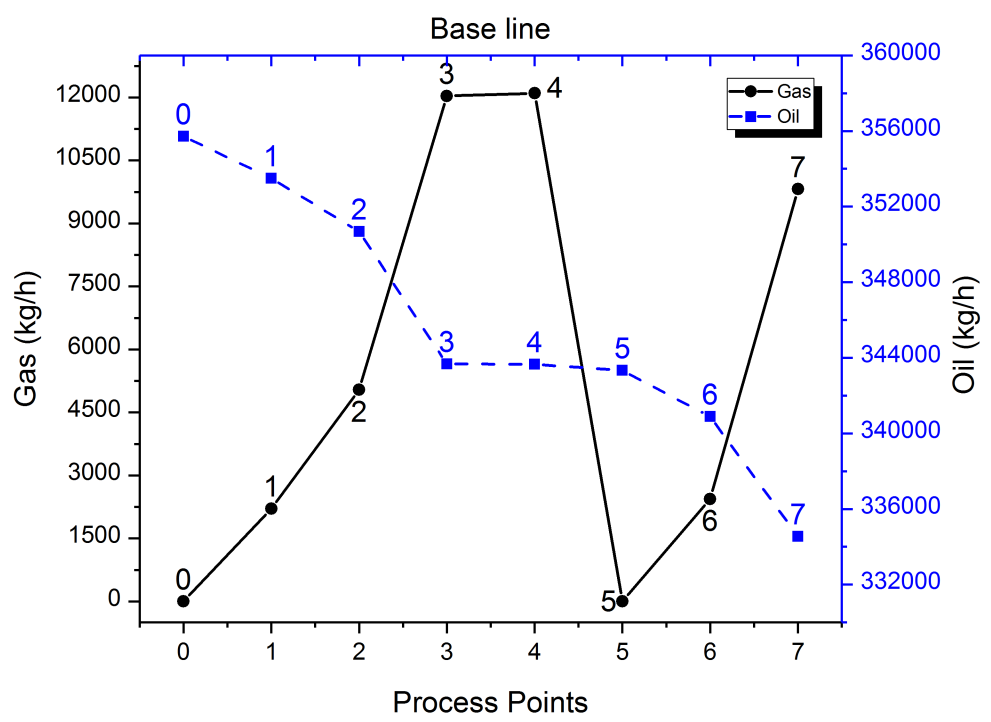

(b)

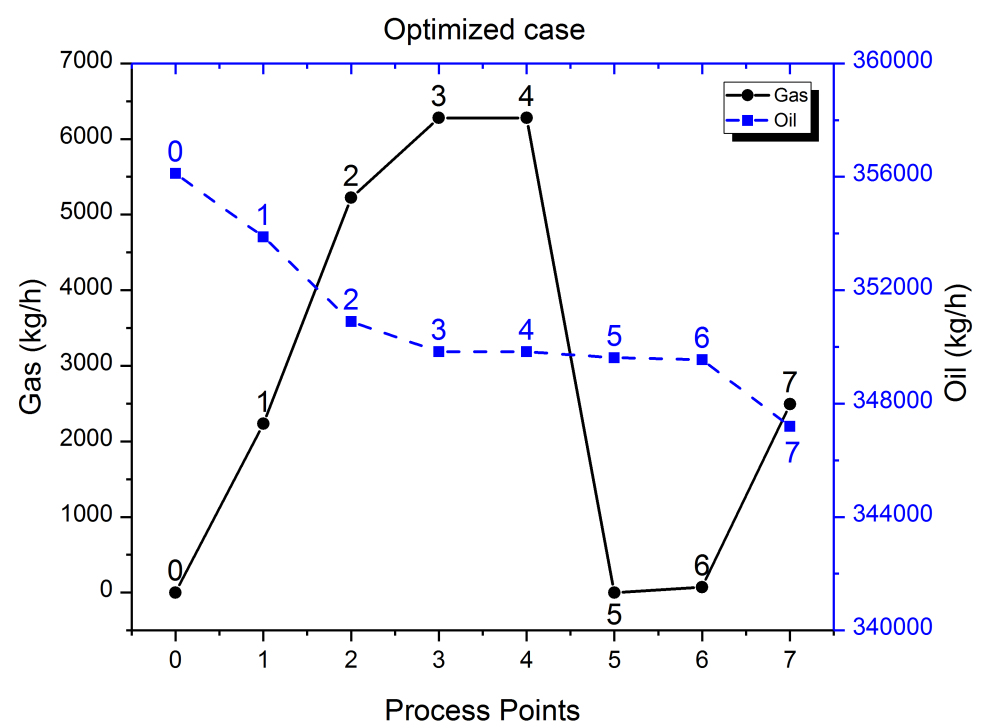

Source : Author 
Table 5.17 allows observing that the optimized case for operation modes 2 leads to an increase of $3.79 \%$ on the FPSO units of exportation oil, in comparison to the baseline. This incremental of liquid mass flow leads to a decrease of power demands of compressors as major electrical energy consumer resulting in a decrease of about $7.7 \%$ of total power demand of proposed FPSO. The separation performance of the optimization case represents a decrease of $1 \%$ while the C3-C5 content of exportation oil has an increase of $9 \%$ as compared to the baseline case of operation mode 2 (Table 5.17).

Table 5.17 - Exportation oil rate, separation performance of all separators and propane, butane and pentane percentage of exportation oil of the FPSO unit for the baseline and optimized case of operation mode 2 .

\begin{tabular}{|c|c|c|c|c|c|c|c|}
\hline & & $\begin{array}{l}\text { Exp. oil } \\
\text { (kg/h) }\end{array}$ & $\%$ & $\begin{array}{c}\text { Sep. Per. } \\
\%\end{array}$ & $\begin{array}{c}\text { C3-C5 } \\
\%\end{array}$ & $\begin{array}{c}\text { Power Con. } \\
\text { (MW) }\end{array}$ & $\%$ \\
\hline \multirow{3}{*}{ Mode 2} & Baseline case & 334517 & \multirow{3}{*}{+3.79} & 70 & 13 & 14.38 & \multirow{3}{*}{-7.7} \\
\hline & & & & & & & \\
\hline & Optimized case & 347200 & & 69 & 22 & 13.27 & \\
\hline
\end{tabular}

\subsubsection{Operation mode 3}

Table 5.18 shows the input parameters for the baseline and the optimized configurations of operation mode 3 with hydrocarbon liquids recovery as the objective function. The DoE design numbers of this optimization algorithm for the initial designs are 350 . In addition, 8000 evaluations are considered as the maximum generations of the optimization algorithm. The convergence of the optimum value of hydrocarbon liquids recovery for operation mode 3 is obtained after approximately 1250 generations (Appendix D, Figure D.6).

Because of very similar crude oil composition among Mode 2 and 3 , the results of optimization is very close to operation mode 2 , except the found value for T2. Table 5.18 shows P2 = $736 \mathrm{kPa}$ and P3 $=450$ of optimization case for operation mode 3 versus, the values for the baseline configuration are $\mathrm{P} 2=428 \mathrm{kPa}$ and $\mathrm{P} 3=232 \mathrm{kPa}$ for operation mode 2 . The effects of input variables are shown in Figures 5.27a \& 5.27b. As the water content of the reservoir in the current operation year is in the maximum state, thus the maximization of hydrocarbon liquids recovery is not compared to other operating years. However, even with this condition, point 3 in the optimization case has about $1500 \mathrm{~kg} / \mathrm{h}$ more oil content as compared to point 3 of the baseline configuration. The effect of P2 appears at p6 that with a decrease in operating pressure by pressure valve, less volatile components are liberated in gas flow for the optimization case; $72 \mathrm{~kg} / \mathrm{h}$ versus $781 \mathrm{~kg} / \mathrm{h}$ of the baseline configuration. Consequently, the results shows that the maximization of hydrocarbons liquid recovery is achieved by increasing the operating pressure of the second and the third stages of the separation train and also by decreasing the operating temperature in oil heater of the separation train for operation mode 3. 
Table 5.18 - Input parameters for the baseline and optimized configurations of operation mode 3-Hydrocarbon liquids recovery case.

\begin{tabular}{ccccc}
\hline & & $\begin{array}{c}\mathrm{P} 2 \\
(\mathrm{kPa})\end{array}$ & $\begin{array}{c}\mathrm{P} 3 \\
(\mathrm{kPa})\end{array}$ & $\begin{array}{c}\mathrm{T} 2 \\
\left({ }^{\circ} \mathrm{C}\right)\end{array}$ \\
\hline \multirow{2}{*}{ Mode 3 } & Baseline case & 428 & 232 & 88 \\
& & & & \\
& Optimized case & 736 & 450 & 49 \\
\hline
\end{tabular}

Figure 5.27 - Stabilization of phase during the separation processes for the baseline and optimized case of operation mode 3 .

(a)

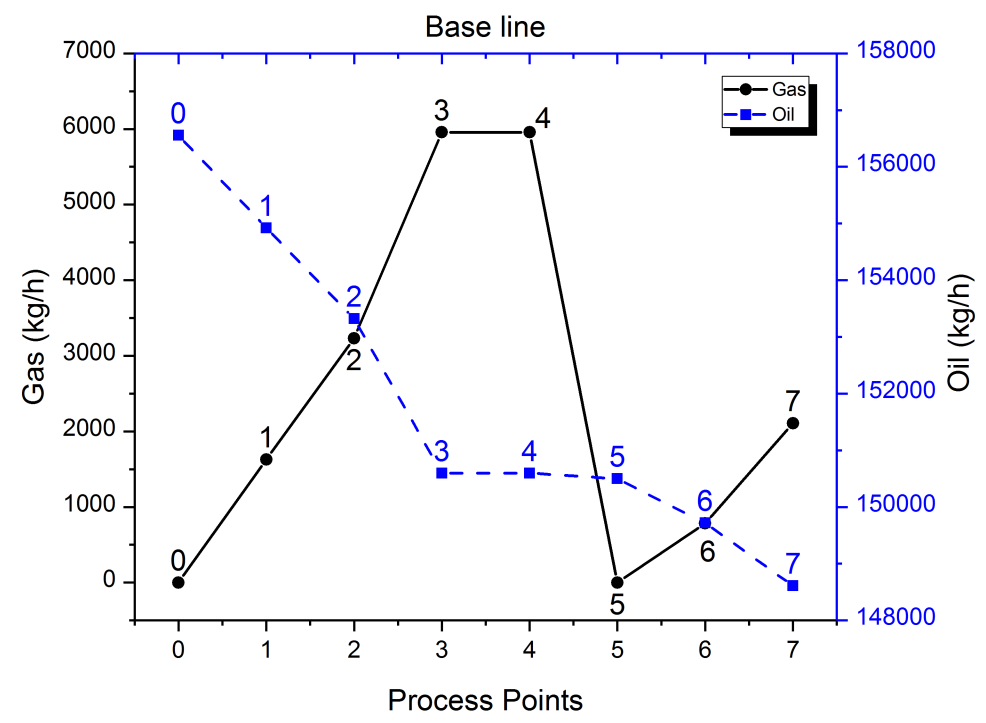

(b)

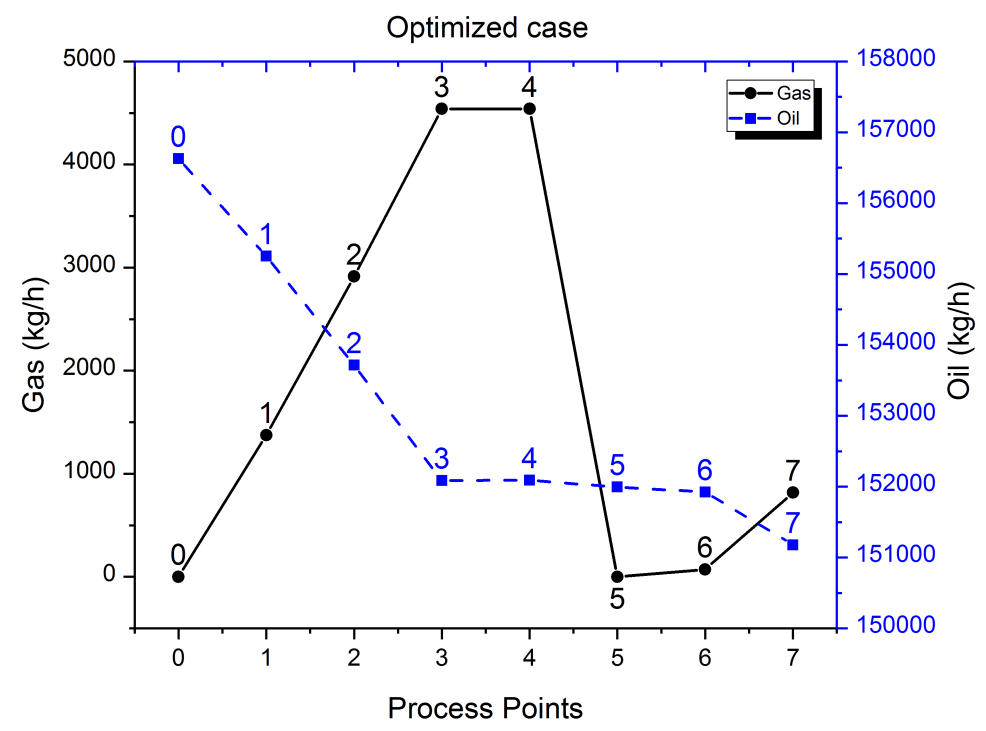

Source : Author 
Table 5.19 shows the calculated Exportation oil rate, performance separation, C3-C5 content of exportation oil and power demand of the FPSO unit for the baseline and optimized cases of operation mode 2 . In that, there is an increase of $1.75 \%$ in exportation oil between the optimization and baseline cases and leads to this increase, there is a less submitted gas flow to compressors resulting a mitigation of $2.9 \%$ in power demand is reported for optimization case. Next, the separation performance of optimization case represents an increase of $3 \%$ while the C3-C5 content of exportation oil is increased $4 \%$ as compared with the baseline case of operation mode 3 (Table 5.19).

Table 5.19 - Exportation oil rate, separation performance of all separators and propane, butane and pentane percentage of exportation oil of the FPSO unit for the baseline and optimized case of operation mode 3.

\begin{tabular}{cccc|c|c|cr}
\hline & & $\begin{array}{c}\text { Exp. oil } \\
(\mathrm{kg} / \mathrm{h})\end{array}$ & $\%$ & $\begin{array}{c}\text { Sep. Per. } \\
\%\end{array}$ & $\begin{array}{c}\text { C3-C5 } \\
\%\end{array}$ & $\begin{array}{c}\text { Power Con. } \\
(\mathrm{MW})\end{array}$ & $\%$ \\
\hline \multirow{2}{*}{ Mode3 } & Baseline case & 148610 & 65 & 6 & 14.72 & -2.9 \\
& Optimized case & 151200 & +1.75 & 68 & 10 & 14.33 & \\
\hline
\end{tabular}




\section{CONCLUSION AND FUTURE WORK}

This chapter presents the conclusion of the current research and the suggestion research activities in the future of this work.

\subsection{Conclusion}

A primary processing plant of a typical FPSO operating in a Brazilian deep-water oil field on pre-salt areas is modeled and simulated using its real operating data. Three operation conditions of the oil field are presented in this research: (i) Maximum oil/gas content (mode 1), (ii) $50 \%$ BSW oil content (mode 2) and (iii) high water $/ \mathrm{CO}_{2}$ in oil content (mode 3). Then, the impact of eight thermodynamic input parameters on fuel consumption and hydrocarbon liquids recovery of the FPSO unit are investigated by the Smoothing Spline ANOVA (SS-ANOVA) method. From SS-ANOVA, the input parameters that presented the highest impact on fuel consumption and hydrocarbons liquids recovery were selected for an optimization procedure.

The optimization procedure consists of a hybrid Genetic Algorithm (NSGA-II+SQP method), which is coupled with the Aspen HYSYS $\AA$ software as the simulator. The objective functions used in the optimization were the minimization of fuel consumption of the processing plant and the maximization of hydrocarbon liquids recovery. The following items are the contributions and novelty of the current thesis:

- Applying the real performance data of a commercial-offshore gas turbine (RB211G62 DLE) with adequate efficiency interacting with simulation of Aspen Hysys (for the first time ever);

- Integrating of Aspen Hysys and ModeForntier as an automation process of screen analyzing and optimization procedure (for the first time ever);

- Implementation of SS-ANOVA (considering the analysis of the main and interaction effects) in an oil and gas processing simulation of proposed FPSO to identify the major effects and to decrease the input variable of optimization procedure;

- Assessment of the available and recommended optimization methods from literature in order to select the suitable one for current problem;

- Applying an optimization procedure to increase the sustainability and profitability of a typical FPSO simultaneously, without adding any new technology and imposed costs;

- Applying a formal optimization procedure for fuel consumption minimization that consequently 
reduces the produced $\mathrm{CO}_{2}$ in the processes and for hydrocarbons liquid recovery, subject to several constraints, of a Brazilian FPSO in three operation scenarios.

From SS-ANOVA, the statistical analysis revealed that the most important parameters affecting the fuel consumption of the plant are: (1)output pressure of the first control valve (P1); (2)output pressure of the second stage of the separation train before mixing with dilution water (P2); (3) input pressure of the third stage of the separation train (P3); (4) output pressure of the main gas compressor (Pc) and; (5) output petroleum temperature in the second heat exchanger (T2). These four input parameters (P1, P2, P3 and Pc), three input parameters (P3, Pc and T2) and three input parameters (P3, Pc and T2) correspond to 96\%, $97 \%$ and $97 \%$ of the total contribution to fuel consumption for modes 1,2 and 3 , respectively.

For hydrocarbon liquids recovery of the plant: These four input parameters (P1, P2, P3 and T2), three input parameters (P3, P2 and T2) and three input parameters (P3, P2 and T2) correspond to $95 \%, 97 \%$ and $98 \%$ of the total contribution to hydrocarbon liquid recovery for modes 1, 2 and 3, respectively. The results showed that P3 is very important to fuel consumption and hydrocarbon liquids recovery due to its impact on separation efficiency and stabilization of the resultant phases of production at the third stage of the separators. Moreover, parameter T2 is more relevant for modes 2 and 3 and its contribution is practically the same for both operation modes. It has an important role in the separation efficiency of wet gas composition at the second stage of the separation train, indicating that it is necessary to provide a heat source to enhance hydrocarbons recovery at the second stage of the separation train.

The implementation of Sensitivity Analysis showed what are the most important parameters that affect the objective function and resulted in a great reduction in the computation time for optimization. For example, the convergence time for the optimization procedure, for some cases, was reduced from 2 weeks to 3 days.

The results from the optimized case indicated that the minimization of fuel consumption is achieved by increasing the operating pressure in the third stage of the separation train and by decreasing the operating temperature in the second stage of the separation train for all operation modes. There was a reduction in power demand of $6.4 \%$ for mode $1,10 \%$ for mode 2 and $2.9 \%$ for mode 3 , in comparison to the baseline case. Consequently, the fuel consumption of the plant was decreased by $4.46 \%$ for mode $1(223 \mathrm{~kg} / \mathrm{h}$ less consumed gas), $8.34 \%$ for mode 2 (262 kg/h less consumed gas) and $2.43 \%$ for mode 3 ( $87 \mathrm{~kg} / \mathrm{h}$ less consumed gas), when compared to the baseline case. Moreover, the optimization producer found an improvement in the recovery of the volatile components, in comparison with the baseline cases.

Furthermore, the optimum operating condition found by the optimization procedure of hydrocarbon liquids recovery presented an increase of $4.36 \%$ for mode 1 (10\% improvement 
of C3-C5 content in exportation oil), 3.79\% for mode 2 (an improvement of 9\% for C3-C5 content in exportation oil) and $1.75 \%$ for mode 3 (an increase of $4 \%$ for C3-C5 content in exportation oil), in hydrocarbons liquid recovery (stabilization and saving), when compared to the conventional operating conditions of their baseline.

\subsection{Future work}

Overall, the optimization process showed to be a robust and promising tool to find optimal operational configurations of an FPSO plant, since the integration between optimization methods and thermodynamic modeling speeds up the evaluation of several configurations automatically.

The operation mode working with high GOR and oil reservoirs have a great potential to be optimized in both objective functions of fuel consumption minimization and hydrocarbon liquid recovery. There are four more real crude oil compositions that can be simulated and used in an optimization procedure.

Moreover, in this research, the separation performance is considered as an indicator to explain the effect of temperatures and pressures on hydrocarbons recovery and stabilization of oil components and the assessment of the optimization results. In future studies, it can be used as an objective function aiming to improve the recovery of hydrocarbons components for each operation mode considering API gravity number. 



\section{REFERENCES}

ABDEL-AAL, H. K.; AGGOUR, M. A.; FAHIM, M. A. Petroleum and Gas Field Processing, Second Edition. 2nd. ed.: CRC Press, 2015. ISBN 9781482255928. Cited 7 times in pages $41,99,101,113,132,157$, and 160.

ADJIMAN, C. S.; SCHWEIGER, C. A.; FLOUDAS, C. A. Mixed-integer nonlinear optimization in process synthesis. In: Handbook of Combinatorial Optimization: Springer US, 1998. p. 1-76. Cited in page 61.

ALAGUMURTHI, N.; PALANIRADJA, K.; SOUNDARARAJAN, V. Optimization of grinding process through design of experiment (doe) - a comparative study. Materials and Manufacturing Processes, Taylor \& Francis, v. 21, n. 1, p. 19-21, 2006. Cited in page 87.

ALLAHYARZADEH-BIDGOLI, A.; DEZAN, D. J.; YANAGIHARA, J. I. PERFORMANCE OPTIMIZATION OF PLATE-FIN HEAT EXCHANGERS USED IN a THREE STAGE PROPANE PRE-COOLING CYCLE OF THE CASCADE LNG PLANT. In: Proceedings of CHT-17 ICHMT International Symposium on Advances in Computational Heat Transfer May 28-June 1, 2017, Napoli, Italy: Begellhouse, 2017. Cited in page 76.

ALLAHYARZADEH-BIDGOLI, A. et al. Optimization of a brazilian fpso fuel consumption for petroleum composition with maximum oil and gas content using genetic algorithm method. In: Proceedings of ECOS 2017-the 30th International Conference on Efficiency, Cost, Optimization, Simulation and Environmental Impact of Energy Systems, 2017. Cited 2 times in pages 71 and 76.

ANANTHARAMAN, R. Energy Efficiency in Process Plants with emphasis on Heat Exchanger Networks Optimization, Thermodynamics and Insight. Thesis (PhD) Department of Energy and Process Engineering Norwegian University of Science and Technology, 2011. Available from Internet: <http://www.ivt.ntnu.no/ept/fag/tep4215/innhold/ Rahul\%20-\%20thesis\%20-\%20final\%20version\%20-\%2030\%20May\%202011.pdf>. Cited in page 85.

ANDRADE, A. M. T. de et al. Offshore production units for pre-salt projects. In: OFFSHORE TECHNOLOGY CONFERENCE. Offshore Technology Conference, 2015. Cited 3 times in pages 49,50 , and 51.

ANSCOMBE, F. J. The validity of comparative experiments. Journal of the Royal Statistical Society. Series A (General), JSTOR, v. 111, n. 3, p. 181, 1948. Cited in page 90.

ANTONIN, P. et al. About the relevance of mathematical programming and stochastic optimisation methods: Application to optimal batch plant design problems. In: Computer Aided Chemical Engineering: Elsevier, 2005. p. 49-54. Cited in page 85.

ANTONIN, P. et al. About the relevance of mathematical programming and stochastic optimisation methods: application to optimal batch plant design problems. Computer Aided Chemical Engineering, Elsevier, v. 20, p. 49-54, 2005. Cited in page 94. 
ARAÚJO, O. de Q. F. et al. Comparative analysis of separation technologies for processing carbon dioxide rich natural gas in ultra-deepwater oil fields. Journal of Cleaner Production, Elsevier BV, v. 155, p. 12-22, jul 2017. Cited in page 33.

ARNOLD, K.; STEWART, M. Design of oil-handling systems and facilities: Gulf Professional Publishing, 1998. Cited in page 46.

ARNOLD, M. S. K. Surface Production Operations, Volume 1: Elsevier Science \& Technology, 2007. ISBN 0750678534. Cited 5 times in pages 42, 43, 44, 45, and 132.

ARORA, J. Introduction to optimum design. 4th edition. ed.: Academic Press, 2016. Cited in page 93.

Aspen Technology, Inc. Aspen Simulation Workbook datasheet, 2014. Cited in page 114.

ASPENTECHNOLOGYINC. Aspen HYSYS V9.0, 2016. Cited in page 113.

AUTE, V. C. Thermal Systems Optimization: Wiley Online Library, 2014. Cited in page 64.

AYRES, R. U.; AYRES, L. W.; MARTINÁS, K. Exergy, waste accounting, and life-cycle analysis. Energy, Elsevier, v. 23, n. 5, p. 355-363, 1998. No citation in text.

BAHADORI, A.; NWAOHA, C.; CLARK, M. W. Dictionary of Oil, Gas, and Petrochemical Processing: CRC PR INC, 2013. ISBN 9781466588257. Cited in page 136.

BAHADORI, A.; VUTHALURU, H. B.; MOKHATAB, S. Optimizing separator pressures in the multistage crude oil production unit. Asia-Pacific Journal of Chemical Engineering, Wiley-Blackwell, v. 3, n. 4, p. 380-386, jul 2008. Cited 2 times in pages 70 and 74.

BALESTIERI, J. A. P. Cogeraçao: Geraçao combinada de electricidade e calor: Editora da UFSC, 2002. Cited in page 33.

BARRERA, J. E.; BAZZO, E.; KAMI, E. Exergy analysis and energy improvement of a brazilian floating oil platform using organic rankine cycles. Energy, Elsevier, v. 88, p. 67-79, 2015. Cited in page 34.

BARTON, R. R.; MECKESHEIMER, M. Chapter 18 metamodel-based simulation optimization. In: Simulation: Elsevier, 2006. p. 535-574. Cited in page 86.

BELTRAO, R. L. C. et al. Ss: Pre-salt santos basin-challenges and new technologies for the development of the pre-salt cluster, santos basin, brazil. In: OFFSHORE TECHNOLOGY CONFERENCE. Offshore Technology Conference, 2009. Cited 2 times in pages 47 and 49.

BIEGLER, L. T.; GROSSMANN, I. E.; WESTERBERG, A. W. Systematic methods for chemical process design. Prentice Hall, Old Tappan, NJ (United States), 1997. ISSN 978-0134924229. ISBN 978-0134924229. Available from Internet: <https://www.ebook.de/de/product/3714872/lorenz_t_biegler_ignacio_e_grossmann_ arthur_w_westerberg_systematic_methods_of_chemical_process_design.html>. Cited in page 96.

BORGONOVO, E. Sensitivity Analysis: Springer International Publishing, 2017. Cited in page 90. 
BOTHAMLEY, M. et al. Offshore processing options for oil platforms. In: SOCIETY OF PETROLEUM ENGINEERS. SPE Annual Technical Conference and Exhibition, 2004. p. 1-17. Cited 2 times in pages 53 and 57.

BP, B.-P. Azari, Chirag \& Gunashli Full Field Development Phase 3. Environmental \& Socio-economic impact assessment,. 2004. Report. Available from Internet: <http: //disciplinas.stoa.usp.br/pluginfile.php/161720/mod_folder/content/0/05\%20Chapt\%205\% 20PD\%20Section\%205.5\%20Process_ENG_FINAL_Oct\%2004.pdf?forcedownload=1 >. Visited on: 05/05/2016. Cited 2 times in pages 46 and 47.

BRADLEY, S.; HAX, A.; MAGNANTI, T. Applied mathematical programming. Addison Wesley, 1977. ISBN 978-0201004649. Available from Internet: <https: //www.amazon.com/Applied-Mathematical-Programming-Stephen-Bradley/dp/ 020100464X? Subscriptionld=0JYN1 NVW651 KCA56C102\&tag=techkie-20\&linkCode= xm2\&camp $=2025 \&$ creative $=165953 \&$ creativeASIN $=020100464 X>$. Cited in page 60 .

CARRANZA-SÁNCHEZ, Y. A.; OLIVEIRA-JR, S. de. Exergy analysis of offshore primary petroleum processing plant with co 2 capture. Energy, Elsevier, v. 88, p. 46-56, 2015. Cited in page 58.

CARRANZA-SÁNCHEZ, Y. A. et al. Energy and exergy performance of three fpso operational modes. In: Proceedings of the $23 \mathrm{rd}$ Abcm International Congress of Mechanical Engineering, 2015. Cited 2 times in pages 55 and 59.

Chisholm, Hugh. 'Petroleum'. 1911 Encyclopedia Britanica. 1911. Available from Internet: <http://www.studylight.org/encyclopedias/bri/view.cgi?number=25685>. Visited on: 01/12/2015. Cited in page 31.

CHU, Y.; HAHN, J. Necessary condition for applying experimental design criteria to global sensitivity analysis results. Computers \& Chemical Engineering, Elsevier, v. 48, p. 280-292, jan 2013. Cited 2 times in pages 64 and 65.

COLMENARES, T. R.; SEIDER, W. D. Synthesis of utility systems integrated with chemical processes. Industrial \& engineering chemistry research, ACS Publications, v. 28, n. 1, p. 84-93, 1989. Cited 2 times in pages 60 and 74.

DEB, K. et al. A fast and elitist multiobjective genetic algorithm: Nsga-ii. IEEE transactions on evolutionary computation, IEEE, v. 6, n. 2, p. 182-197, 2002. Cited 2 times in pages 94 and 95.

DEVOLD, H. Oil and gas production handbook: an introduction to oil and gas production: $A A B, 2006$. Cited 2 times in pages 47 and 102.

DEZAN, D. J. HEAT TRANSFER ENHANCEMENT AND OPTIMIZATION OF FLAT-TUBE MULTILOUVERED FIN COMPACT HEAT EXCHANGERS WITH DELTAWINGLET VORTEX GENERATORS. Thesis (PhD) - Universidade de Sao Paulo, 2015. Cited in page 92.

DIBAN, P.; FOO, D. C. Targeting and design of heating utility system for offshore platform. Energy, Elsevier, v. 146, p. 98-111, mar 2017. Cited 2 times in pages 63 and 76.

EFRON, B.; STEIN, C. The jackknife estimate of variance. The Annals of Statistics, JSTOR, p. 586-596, 1981. Cited in page 91. 
Esteco SPA. ModeFRONTIER - User's Guide Version 5.6.0.1 2017, 2017. Cited 7 times in pages $90,95,114,147,148,149$, and 150.

\section{ESTECO WEBINAR SERIES. LEVERAGING SMART DESIGN EXPLORATION FOR RESOURCE-INTENSIVE CFD ANALYSIS. 2016. Cited in page 90.}

ETA-OFFSHORE-SEMINARS, i. The technology of offshore drilling, completion and production: Pennwell Corp, 1976. Cited in page 46.

FANG, K.-T.; LI, R.; SUDJIANTO, A. Design and modeling for computer experiments: CRC Press, 2005. Cited in page 87.

FAZLOLLAHI, S. et al. Multi-objective, multi-period optimization of district energy systems: Iv-a case study. Energy, Elsevier, v. 84, p. 365-381, 2015. Cited in page 85.

FENG, Y. et al. Comparison between regenerative organic rankine cycle (rorc) and basic organic rankine cycle (borc) based on thermoeconomic multi-objective optimization considering exergy efficiency and levelized energy cost (lec). Energy Conversion and Management, Elsevier, v. 96, p. 58-71, 2015. Cited 3 times in pages 67, 68, and 75.

FERGANI, Z.; TOUIL, D.; MOROSUK, T. Multi-criteria exergy based optimization of an organic rankine cycle for waste heat recovery in the cement industry. Energy Conversion and Management, Elsevier, v. 112, p. 81-90, 2016. Cited in page 68.

FISHER, R. A.; MACKENZIE, W. A. Studies in crop variation. ii. the manurial response of different potato varieties. The Journal of Agricultural Science, Cambridge University Press, v. 13, n. 3, p. 311-320, 1923. Cited in page 91.

FISHER, S. R. A. et al. The design of experiments: Oliver and Boyd Edinburgh, 1937. v. 1. Cited in page 86.

FONTAINE, E. et al. Reliability analysis and response based design of a moored fpso in west africa. Structural Safety, Elsevier, v. 41, p. 82-96, 2013. Cited in page 34.

FORMIGLI, J. Pre-salt reservoirs offshore brazil: perspectives and challenges. In: Energy Conference. Miami, 2007. p. 12-23. Cited in page 48.

FRANGOPOULOS, C. A.; SPAKOVSKY, M. R. V.; SCIUBBA, E. A brief review of methods for the design and synthesis optimization of energy systems. International Journal of Thermodynamics, v. 5, n. 4, p. 151-160, 2002. Cited in page 94.

GALLO, W. L. et al. Exergy analysis of the compression systems and its prime movers for a FPSO unit. Journal of Natural Gas Science and Engineering, Elsevier BV, v. 44, p. 287-298, Aug 2017. Cited 6 times in pages 55, 56, 59, 79, 80, and 113.

$\mathrm{GAO}$, T. et al. Coalbed methane liquefaction adopting a nitrogen expansion process with propane pre-cooling. Applied Energy, Elsevier BV, v. 87, n. 7, p. 2142-2147, jul 2010. Cited in page 64.

GEHLING, R. C.; LANE, M. P.; THORNTON, R. M. Floating production, storage and offloading (fpso) facilities-impact of the new oil tanker construction rules. APEA JOURNAL, Australian Petroleum Exploration Association, v. 34, p. 178-178, 1994. Cited in page 33. 
General Electric. Gate Cycle V6.1.2. New York, USA, 2013. Cited 2 times in pages 97 and 109.

GETU, M. et al. Risk-based optimization for representative natural gas liquid (ngl) recovery processes by considering uncertainty from the plant inlet. Journal of Natural Gas Science and Engineering, Elsevier, v. 27, p. 42-54, 2015. Cited in page 68.

GHAEDI, M.; EBRAHIMI, A. N.; PISHVAIE, M. R. APPLICATION OF GENETIC ALGORITHM FOR OPTIMIZATION OF SEPARATOR PRESSURES IN MULTISTAGE PRODUCTION UNITS. Chemical Engineering Communications, Informa UK Limited, v. 201, n. 7, p. 926-938, mar 2014. Cited in page 70.

GIUNTA, A. A. et al. Overview of modern design of experiments methods for computational simulations. In: Proceedings of the 41st AIAA aerospace sciences meeting and exhibit, AIAA-2003-0649, 2003. Cited in page 87.

GONG, M. Using exergy and optimization models to improve industrial energy systems towards sustainability. Linköpings universitet, 2004. Cited in page 84.

GROSSMANN, I. E. Mixed-integer programming approach for the synthesis of integrated process flowsheets. Computers \& chemical engineering, Elsevier, v. 9, n. 5, p. 463-482, 1985. Cited 3 times in pages 83, 85, and 96.

GROSSMANN, I. E. Global Optimization in engineering design: Springer Science \& Business Media, 2013. v. 9. Cited in page 84.

GU, C. Smoothing Spline ANOVA Models: Springer New York, 2013. Cited in page 91.

GUERRA, M. J. Aspen HYSYS Property Packages-Overview and Best Practices for Optimum Simulations. 2006. Pdf. Available from Internet: <http://sites.poli.usp.br/d/pqi2408/ BestPracticesOptimumSimulationsHYSYSPropertyPackages.pdf>. Cited in page 116.

HAFTKA, R. T.; GÜRDAL, Z. Elements of structural optimization: Springer Science \& Business Media, 2012. v. 11. Cited in page 84.

HALLIBURTON. Água profundas - Brasil. 2014. Available from Internet: <http: //www.halliburton.com/public/solutions/contents/Deep_Water/related_docs/Brazil\% 20Deepwater\%20Map\%20Final\%20-\%20Portuguese.pdf>. Visited on: 07/05/2016. Cited in page 34.

HAMID, M. K. A. Hysys $\AA$ : An introduction to chemical engineering simulation. Apostila de Hamid, 2007. Cited in page 116.

HATCHER, P.; KHALILPOUR, R.; ABBAS, A. Optimisation of Ing mixed-refrigerant processes considering operation and design objectives. Computers \& Chemical Engineering, Elsevier, v. 41, p. 123-133, jun 2012. Cited in page 64.

HE, T.; JU, Y. Design and optimization of a novel mixed refrigerant cycle integrated with ngl recovery process for small-scale Ing plant. Industrial \& Engineering Chemistry Research, ACS Publications, v. 53, n. 13, p. 5545-5553, 2014. Cited 2 times in pages 66 and 68.

HE, T.; JU, Y. Optimal synthesis of expansion liquefaction cycle for distributed-scale Ing (liquefied natural gas) plant. Energy, Elsevier, v. 88, p. 268-280, 2015. Cited 2 times in pages 66 and 68. 
HOEFFDING, W. A class of statistics with asymptotically normal distribution. The annals of mathematical statistics, JSTOR, p. 293-325, 1948. Cited in page 91.

HUANG, K.; KARIMI, I. Work-heat exchanger network synthesis (whens). Energy, Elsevier, v. 113, p. 1006-1017, oct 2016. Cited in page 75.

HWANG, J.-H.; ROH, M.-I.; LEE, K.-Y. Determination of the optimal operating conditions of the dual mixed refrigerant cycle for the LNG FPSO topside liquefaction process. Computers \& Chemical Engineering, Elsevier BV, v. 49, p. 25-36, feb 2013. Cited in page 65.

HYPROTECH-LTD. HYSIM User's Guide - Version C1.50, 1991. Cited in page 52.

INUTECH-GMBH. Mipsqp - a new approach to solve mixed integer optimization problems. dez. 2007. Cited in page 85.

IOGP, I. A. O. O. . G. P. Environmental performance indicators 2011 data - International Association of Oil \& Gas Producers, London, October 2012. 2012. Available from Internet: <www.ogp.org.uk>. Visited on: 14/05/2016. Cited in page 54.

IUPAC. International union of pure and applied chemistry organic chemistry division.a guide to iupac nomenclature of organic compounds (recommendations 1993). Blackwell Scientific Publications, 1993. Cited in page 41.

JALURIA, Y. Design and optimization of thermal systems: CRC press, 2007. Cited 2 times in pages 84 and 85.

JIANG, X.; LI, S.; ZHANG, L. Sensitivity analysis of gas production from class i hydrate reservoir by depressurization. Energy, Elsevier, v. 39, n. 1, p. 281-285, mar 2012. Cited 2 times in pages 64 and 65.

KHAN, M. S. et al. Energy saving opportunities in integrated $\mathrm{ngl} / \mathrm{lng}$ schemes exploiting: Thermal-coupling common-utilities and process knowledge. Chemical Engineering and Processing: Process Intensification, Elsevier, v. 82, p. 54-64, 2014b. Cited 2 times in pages 66 and 68.

KHAN, M. S. et al. Process knowledge based opportunistic optimization of the $\mathrm{n} 2-\mathrm{co} 2$ expander cycle for the economic development of stranded offshore fields. Journal of Natural Gas Science and Engineering, Elsevier, v. 18, p. 263-273, 2014a. Cited 2 times in pages 65 and 68.

KHERAD-PAJOUH, S.; RENAUD, O. An exact permutation method for testing any effect in balanced and unbalanced fixed effect ANOVA. Computational Statistics \& Data Analysis, Elsevier BV, v. 54, n. 7, p. 1881-1893, jul 2010. Cited in page 91.

$\mathrm{KIM}$, I. H. et al. Simulation-based optimization of multistage separation process in offshore oil and gas production facilities. Industrial \& Engineering Chemistry Research, American Chemical Society (ACS), v. 53, n. 21, p. 8810-8820, may 2014. Cited 5 times in pages 43, $70,75,157$, and 160.

KINNEY P.E., D. B. PF and C: Expanding Facilities Knowledge Workshop Session 1 - Field Development Options / Selection Strategy. 2012. Available from Internet: <http://www.spegcs.org/events/1984/>. Visited on: 05/04/2016. Cited in page 33. 
KLOSTER, P. et al. Energy optimization on offshore installations with emphasis on offshore combined cycle plants. In: SOCIETY OF PETROLEUM ENGINEERS. Offshore Europe Oil and Gas Exhibition and Conference, 1999. Cited in page 74.

KLOSTER, P. et al. Reduction of emissions to air through energy optimisation on offshore installations. In: SOCIETY OF PETROLEUM ENGINEERS. SPE International Conference on Health, Safety and Environment in Oil and Gas Exploration and Production, 2000. Cited in page 74.

KWAK, D.-H. et al. Energy-efficient design and optimization of boil-off gas (BOG) re-liquefaction process for liquefied natural gas (LNG)-fuelled ship. Energy, Elsevier BV, v. 148, p. 915-929, apr 2018. Cited 2 times in pages 68 and 69.

KYLLING, $\varnothing$. W. Optimizing separator pressure in a multistage crude oil production plant. Dissertação (Mestrado) - Norwegian University of Science and Technology-Department of Engineering Cybernetics, 2009. Cited in page 70.

LAZZARETTO, A.; TSATSARONIS, G. Speco: a systematic and general methodology for calculating efficiencies and costs in thermal systems. Energy, Elsevier, v. 31, n. 8, p. 1257-1289, 2006. Cited in page 96.

LEARY, S.; BHASKAR, A.; KEANE, A. Optimal orthogonal-array-based latin hypercubes. Journal of Applied Statistics, Taylor \& Francis, v. 30, n. 5, p. 585-598, 2003. Cited in page 89.

LEE, G.; SMITH, R.; ZHU, X. Optimal synthesis of mixed-refrigerant systems for low-temperature processes. Industrial \& engineering chemistry research, ACS Publications, v. 41 , n. 20 , p. 5016-5028, 2002. Cited 2 times in pages 61 and 74.

LIU, X. et al. Thermal performance analysis of brayton cycle with waste heat recovery boiler for diesel engines of offshore oil production facilities. Applied Thermal Engineering, Elsevier, 2016. Cited in page 72.

LOUREIRO, S.; ROVERE, E.; MAHLER, C. Analysis of potential for reducing emissions of greenhouse gases in municipal solid waste in brazil, in the state and city of rio de janeiro. Waste management, Elsevier, v. 33, n. 5, p. 1302-1312, 2013. Cited in page 31.

LUCAS, M. A. et al. Use of real crude oil fractions to describe the high pressure phase behavior of crude oil in carbon dioxide. The Journal of Supercritical Fluids, Elsevier BV, v. 118 , p. $140-147$, dec 2016. Cited in page 80.

LUCAY, F.; CISTERNAS, L.; GÁLVEZ, E. Global sensitivity analysis for identifying critical process design decisions. Chemical Engineering Research and Design, Elsevier, v. 103, p. 74-83, nov 2015. Cited in page 64.

MANNING, F. S.; THOMPSON, R. E. Oilfield processing of petroleum: Crude oil: Pennwell books, 1995. v. 2. Cited 2 times in pages 52 and 57.

MARÉCHAL, F.; KALITVENTZEFF, B. Targeting the optimal integration of steam networks: Mathematical tools and methodology. Computers \& Chemical Engineering, Elsevier, v. 23, p. S133-S136, 1999. Cited in page 123.

MCCAIN, W. D.; SPIVEY, J. P.; LENN, C. P. Petroleum reservoir fluid property correlations: PennWell Books, 2011. Cited 4 times in pages 42, 132, 136, and 155. 
MCKAY, M. D.; BECKMAN, R. J.; CONOVER, W. J. A comparison of three methods for selecting values of input variables in the analysis of output from a computer code. Technometrics, Taylor \& Francis Group, v. 42, n. 1, p. 55-61, 2000. Cited 2 times in pages 88 and 89.

MEHRPOOYA, M. et al. Energy and advanced exergy analysis of an existing hydrocarbon recovery process. Energy Conversion and Management, Elsevier, v. 123, p. 523-534, 2016. Cited 3 times in pages 55, 59, and 68.

Minerals Management Service Gulf of Mexico OCS Region. Proposed Use of Floating Production, Storage, and Offloading Systems On the Gulf of Mexico Outer Continental Shelf: U.S. Department of the Interior, Minerals Management Service Gulf of Mexico OCS Region, 2000. Cited in page 49.

Ministério de Minas e Energia. Resenha Energética Brasileira Ministério de Minas e Energia Exercício de 2014 - Edição de junho de 2015. 2015. Available from Internet: <http://www.mme.gov.br/documents/1138787/1732840/Resenha+Energ\%C3\%A9tica+-+ Brasil+2015.pdf/4e6b9a34-6b2e-48fa-9ef8-dc7008470bf2>. Visited on: 05/05/2016. Cited in page 31.

Ministério de Minas e Energia. Resenha Energética Brasileira Ministério de Minas e Energia Exercício de 2016 - Edição de junho de 2017. 2017. Available from Internet: <http://www. mme.gov.br/documents/10584/3580498/02+-+Resenha+Energ\%C3\%A9tica+Brasileira+ 2017+-+ano+ref.+2016+\%28PDF\%29/13d8d958-de50-4691-96e3-3ccf53f8e1e4? version=1.0 > Visited on: 05/01/2018. Cited in page 48 .

MISHRA, M.; DAS, P.; SARANGI, S. Second law based optimisation of crossflow plate-fin heat exchanger design using genetic algorithm. Applied Thermal Engineering, Elsevier, v. 29, n. 14, p. 2983-2989, 2009. Cited in page 94.

MONTGOMERY, D. C. Design and analysis of experiments. 4th. ed.: John Wiley \& Sons, 1997. Cited in page 88.

MORAIS, J. M. d. Petróleo em águas profundas: uma história tecnológica da petrobras na exploração e produção offshore. Instituto de Pesquisa Econômica Aplicada (Ipea), 2013. Cited 3 times in pages 34, 35, and 36.

MOSAFFA, A.; MOKARRAM, N. H.; FARSHI, L. G. Thermo-economic analysis of combined different ORCs geothermal power plants and LNG cold energy. Geothermics, Elsevier BV, v. 65 , p. 113-125, jan 2017. Cited in page 67.

MUELLER, W.; ROOBAERT, N. Standardization adds value to fpso topsides. Offshore, Pennwell, v. 68, n. 3, 2008. Cited 2 times in pages 49 and 50.

MYERS, R. H.; MONTGOMERY, D. C.; ANDERSON-COOK, C. M. Response surface methodology: process and product optimization using designed experiments: John Wiley \& Sons, 2016. Cited in page 89.

NGUYEN, T.-V. et al. Life performance of oil and gas platforms: Site integration and thermodynamic evaluation. Energy, Elsevier, v. 73, p. 282-301, 2014b. Cited 2 times in pages 55 and 58. 
NGUYEN, T.-V.; OLIVEIRA-JR, S. de. Optimisation of preliminary systems designs for offshore oil and gas production. In: 4th International Conference on Contemporary Problems of Thermal Engineering CPOTE 2016, 14-16 September 2016, Katowice, Poland Institute of Thermal Technology, 2016g. Cited 3 times in pages 71, 76, and 160.

NGUYEN, T.-V.; OLIVEIRA-JR, S. de. Thermodynamic analysis of oil and gas platforms over various production profiles and feed compositions. In: 30th International Conference on Efficiency, Cost, Optimization, Simulation and Environmental Impact of Energy SystemsInternational Conference on Efficiency, Cost, Optimization, Simulation and Environmental Impact of Energy Systems, 2017. Cited 2 times in pages 55 and 59.

NGUYEN, T.-V. et al. Exergetic assessment of energy systems on north sea oil and gas platforms. Energy, Elsevier, v. 62, p. 23-36, 2013. Cited 5 times in pages 32, 33, 47, 54, and 58.

NGUYEN, T.-V.; SIN, G.; ELMEGAARD, B. Synthesis of preliminary system designs for offshore oil and gas production. In: ELSEVIER SCIENCE. Proceedings of the 26th European Symposium on Computer Aided Process Engineering, 2016f. Cited in page 63.

NGUYEN, T.-V. et al. Co 2-mitigation options for the offshore oil and gas sector. Applied Energy, Elsevier, v. 161, p. 673-694, 2016e. Cited 2 times in pages 72 and 75.

NGUYEN, T.-V. et al. Energy efficiency measures for offshore oil and gas platforms. Energy, Elsevier, 2016a. Cited 2 times in pages 72 and 75.

NGUYEN, T.-V. et al. On the definition of exergy efficiencies for petroleum systems: Application to offshore oil and gas processing. Energy, Elsevier, v. 73, p. 264-281, 2014a. Cited in page 55.

NISHIDA, N.; STEPHANOPOULOS, G.; WESTERBERG, A. W. A review of process synthesis. AlChE Journal, Wiley Online Library, v. 27, n. 3, p. 321-351, 1981. Cited in page 60.

NUNES, G. C. et al. Petrobras approach to fpso cost reduction. In: OFFSHORE TECHNOLOGY CONFERENCE. Offshore Technology Conference, 2016. Cited 2 times in pages 51 and 52 .

OFFSHORE CENTER DENMARK. Overview of the Brazilian oil and gas industry. 2009. Internet. Available from Internet: <http://www.offshorecenter.dk/filer/files/Project/ Internationalisering/OCD\%20report\%20Brazil.pdf>. Visited on: 15/05/2017. Cited in page 49.

OLIVEIRA-JR., S. D.; HOMBEECK, M. V. Exergy analysis of petroleum separation processes in offshore platforms. Energy Conversion and Management, Elsevier, v. 38, n. 15, p. 1577-1584, 1997. Cited 3 times in pages 52, 55, and 57.

OWEN, A. B. The dimension distribution and quadrature test functions. Statistica Sinica, JSTOR, p. 1-17, 2003. Cited in page 91.

OYAMA, A. Wing design using evolutionary algorithms. Thesis (PhD) - Tohoku University, 2000. Cited in page 94. 
PAPOULIAS, S. A.; GROSSMANN, I. E. A structural optimization approach in process synthesis - iii: Total processing systems. Computers \& chemical engineering, Elsevier, v. 7, n. 6, p. $723-734,1983$. Cited in page 83.

PARETO, V. Manuale di economia politica: Societa Editrice, 1906. v. 13. No citation in text.

PARK, J. H. et al. Techno-economic evaluation of a novel ngl recovery scheme with nine patented schemes for offshore applications. Journal of Natural Gas Science and Engineering, Elsevier, v. 27, p. 2-17, 2015. Cited in page 67.

PARK, J. H.; KHAN, M. S.; LEE, M. Modified coordinate descent methodology for solving process design optimization problems: application to natural gas plant. Journal of Natural Gas Science and Engineering, Elsevier, v. 27, p. 32-41, 2015a. Cited in page 67.

PB, P. B. Statistical review of world energy 2015. BP, London, 2015. Cited in page 31.

PENG, D.-Y.; ROBINSON, D. B. A new two-constant equation of state. Industrial \& Engineering Chemistry Fundamentals, American Chemical Society (ACS), v. 15, n. 1, p. 59-64, feb 1976. Cited 3 times in pages 79, 113, and 116.

PetRobRAs, U. P. E. d. C. e. T. E. e. P. PROCESSAMENTO Primeiro de Petróleo. 2007. Available from Internet: <https://engenhariaquimica.files.wordpress.com/2010/04/ apostila-ppp.pdf>. Visited on: 25/05/2016. Cited in page 35.

PHAM, T. N. et al. Optimization of modified single mixed refrigerant process of natural gas liquefaction using multivariate coggin's algorithm combined with process knowledge. Journal of Natural Gas Science and Engineering, Elsevier, 2016. Cited in page 68.

PIEROBON, L. et al. Waste heat recovery technologies for offshore platforms. Applied Energy, Elsevier, v. 136, p. 228-241, 2014b. Cited in page 71.

PIEROBON, L.; HAGLIND, F. Design and optimization of air bottoming cycles for waste heat recovery in off-shore platforms. Applied Energy, Elsevier, v. 118, p. 156-165, 2014a. Cited 2 times in pages 71 and 75.

PIEROBON, L. et al. Multi-objective optimization of organic rankine cycles for waste heat recovery: Application in an offshore platform. Energy, Elsevier, v. 58, p. 538-549, 2013. Cited 2 times in pages 71 and 75.

PINTO, C. et al. An evaluation of large capacity processing units for ultra deep water and high gor oil fields. In: OFFSHORE TECHNOLOGY CONFERENCE. Offshore Technology Conference, 2014. Cited 3 times in pages 49, 50, and 51.

POPOV, A. Genetic algorithms for optimization. User Manual, Hamburg, v. 2013, 2005.

Cited 2 times in pages 93 and 94.

PROCESSONLINE.COM.AU. Using electrical control valve actuators on oil and gas separators. 2014. Report. Available from Internet: <http://www.processonline.com.au/content/process-control-systems/article/ using-electrical-control-valve-actuators-on-oil-and-gas-separators-75422284>. Visited on: 30/05/2016. Cited in page 35.

RAO, S. S. Engineering optimization: theory and practice: John Wiley \& Sons, 2009. Cited 3 times in pages 81, 82, and 123. 
REAY, D.; RAMSHAW, C.; HARVEY, A. Process Intensification: Engineering for efficiency, sustainability and flexibility: Butterworth-Heinemann, 2013. Cited in page 32.

REIS, M. M. L.; GALLO, W. L. Study of waste heat recovery potential and optimization of the power production by an organic rankine cycle in an FPSO unit. Energy Conversion and Management, Elsevier BV, v. 157, p. 409-422, Feb 2018. Cited 4 times in pages 73, 76, 79, and 80.

RICCO, L.; RIGONI, E.; TURCO, A. Smoothing spline anova for variable screening. Dolomites Research Notes on Approximation, v. 6, n. Special_Issue, 2013. Cited in page 91.

RIGONI, E.; RICCO, L. Smoothing spline ANOVA for variable screening, 2011. Cited in page 92.

RIVERO, R. Application of the exergy concept in the petroleum refining and petrochemical industry. Energy conversion and Management, Elsevier, v. 43, n. 9, p. 1199-1220, 2002. Cited in page 57.

RIVERO, R.; RENDON, C.; MONROY, L. The exergy of crude oil mixtures and petroleum fractions: calculation and application. International Journal of Thermodynamics, v. 2, n. 3, p. 115-123, 1999. Cited in page 57.

RUDD, D. F.; POWERS, G. J.; SIIROLA, J. J. Process synthesis: Prentice-Hall, 1973. Cited in page 60.

SALVIANO, L. O.; DEZAN, D. J.; YANAGIHARA, J. I. Thermal-hydraulic performance optimization of inline and staggered fin-tube compact heat exchangers applying longitudinal vortex generators. Applied Thermal Engineering, Elsevier BV, v. 95, p. 311-329, feb 2016. Cited in page 148.

SÁNCHEZ, Y. A. C. Exergy and environmental assessment of FPSO offshore platforms with CO2 capture and storage. Thesis (PhD) - Universidade de São Paulo, 2017. Cited in page 50.

SANTOS, M. G. et al. Natural gas dehydration by molecular sieve in offshore plants: Impact of increasing carbon dioxide content. Energy Conversion and Management, Elsevier BV, v. 149 , p. 760-773, oct 2017. Cited in page 80.

SEPÚLVEDA, F. D.; CISTERNAS, L. A.; GÁLVEZ, E. D. The use of global sensitivity analysis for improving processes: Applications to mineral processing. Computers \& Chemical Engineering, Elsevier BV, v. 66, p. 221-232, jul 2014. Cited 2 times in pages 64 and 65.

SHIMAMURA, Y. Fpso/fso: State of the art. Journal of marine science and technology, Springer, v. 7, n. 2, p. 59-70, 2002. Cited in page 34.

SILVA, T. L. et al. Modeling of flow splitting for production optimization in offshore gas-lifted oil fields: Simulation validation and applications. Journal of Petroleum Science and Engineering, Elsevier, v. 128, p. 86-97, 2015. Cited in page 62.

SMITH, R. Chemical process design: Wiley Online Library, 2005. Cited 3 times in pages 61, 84 , and 85. 
SOBOL, I.; KHAARA, M. kvadraturnye formuly i funktsii. Multidimensional quadrature formulas and functions Haar: Nauka, Moscow, USSR, 1969. Cited in page 91.

SOBOL, I. M. Sensitivity estimates for nonlinear mathematical models. Mathematical modelling and computational experiments, v. 1, n. 4, p. 407-414, 1993. Cited in page 91.

SOFFIATO, M. et al. Design optimization of ORC systems for waste heat recovery on board a LNG carrier. Energy Conversion and Management, Elsevier BV, v. 92, p. 523-534, mar 2015. Cited in page 67.

SUMAN, B. Study of simulated annealing based algorithms for multiobjective optimization of a constrained problem. Computers \& chemical engineering, Elsevier, v. 28, n. 9, p. 1849-1871, 2004. No citation in text.

SVALHEIM, S.; KING, D. C. Life of field energy performance. In: SOCIETY OF PETROLEUM ENGINEERS. Offshore Europe, 2003. Cited in page 53.

SVALHEIM, S. M. et al. Environmental regulations and measures on the norwegian continental shelf. In: SOCIETY OF PETROLEUM ENGINEERS. SPE International Conference on Health, Safety and Environment in Oil and Gas Exploration and Production, 2002. Cited in page 57.

TERRY, R. E.; ROGERS, J. B. Applied Petroleum Reservoir Engineering (3rd Edition): Prentice Hall, 2014. ISBN 978-0133155587. Cited in page 136.

THEVENIN, D.; JANIGA, G. Optimization and computational fluid dynamics. Springer-Verlag Berlin Heidelberg, 2008. Available from Internet: <http://www.springer.com/us/book/ 9783540721529\#aboutBook>. Cited in page 81.

URBAN, N. M.; FRICKER, T. E. A comparison of latin hypercube and grid ensemble designs for the multivariate emulation of an earth system model. Computers \& Geosciences, Elsevier, v. 36, n. 6, p. 746-755, 2010. Cited in page 88.

U.S. ENERGY INFORMATION ADMINISTRATION. Annual energy outlook 2013. US Energy Information Administration, Washington, DC, 2013. Cited in page 31.

U.S. ENERGY INFORMATION ADMINISTRATION. International energy statistics. 2015b. Internet. Available from Internet: <http://www.eia.gov/countries/cab.cfm?fips=br>. Visited on: 07/05/2016. Cited in page 49.

U.S. ENERGY INFORMATION ADMINISTRATION. Offshore production nearly 30of global crude oil output in 2015. 2016. Report. Available from Internet: <https://www.eia.gov/todayinenergy/detail.php?id=28492>. Cited 2 times in pages 45 and 48.

U.S. Energy Information Administration (EIA). Annual Energy Outlook 2018 with projections to 2050. 2018. Available from Internet: <https://www.eia.gov/outlooks/aeo/pdf/AEO2018.pdf>. Cited in page 31.

VANNER, R. Energy use in offshore oil and gas production: trends and drivers for efficiency from 1975 to 2025. Policy Studies Institute (PSI) Working Paper, September, 2005. Cited 2 times in pages 53 and 58. 
VERMA, S. K.; FENILA, F.; SHASTRI, Y. Sensitivity analysis and stochastic modelling of lignocellulosic feedstock pretreatment and hydrolysis. Computers \& Chemical Engineering, Elsevier BV, v. 106, p. 23-39, nov 2017. Cited in page 64.

VOLDSUND, M. et al. Exergy analysis of the oil and gas separation processes on a north sea oil platform. Proceedings of 23th International conference on efficiency, cost, optimization, simulation and environmental impact of energy systems - ECOS 2010, p. 303-310, 2010. Cited 3 times in pages 53, 54, and 58.

VOLDSUND, M. et al. Evaluation of the oil and gas processing at a real production day on a north sea oil platform using exergy analysis. Proceedings of 25th International conference on efficiency, cost, optimization, simulation and environmental impact of energy systems ECOS 2012, p. 153-166, 2012. Cited in page 54.

VOLDSUND, M. et al. Comparative study of the sources of exergy destruction on four north sea oil and gas platforms. In: CHINESE SOCIETY OF ENGINEERING THERMOPHYSICS. Proceedings of Ecos 2013-the 26th International Conference on Efficiency, Cost, Optimization, Simulation and Environmental Impact of Energy Systems, 2013, 2013b. Cited in page 54.

VOLDSUND, M. et al. Performance indicators for evaluation of north sea oil and gas platforms. In: CHINESE SOCIETY OF ENGINEERING THERMOPHYSICS. Proceedings of Ecos 2013-the 26th International Conference on Efficiency, Cost, Optimization, Simulation and Environmental Impact of Energy Systems, 2013, 2013c. Cited in page 58.

VOLDSUND, M. et al. Exergy destruction and losses on four north sea offshore platforms: A comparative study of the oil and gas processing plants. Energy, Elsevier, v. 74, p. 45-58, 2014. Cited 2 times in pages 55 and 58.

WAHBA, G. Improper priors, spline smoothing and the problem of guarding against model errors in regression. Journal of the Royal Statistical Society. Series B (Methodological), JSTOR, p. 364-372, 1978. Cited in page 91.

WAHBA, G. et al. Smoothing spline anova for exponential families, with application to the wisconsin epidemiological study of diabetic retinopathy: the 1994 neyman memorial lecture. The Annals of Statistics, Institute of Mathematical Statistics, v. 23, n. 6, p. 1865-1895, 1995. Cited in page 91.

WANG, L. et al. Superstructure-free synthesis and optimization of thermal power plants. Energy, Elsevier, v. 91, p. 700-711, nov 2015. Cited in page 62.

WANG, L. et al. Systematic optimization of the design of steam cycles using minlp and differential evolution. Journal of Energy Resources Technology, American Society of Mechanical Engineers, v. 136, n. 3, p. 031601, mar 2014. Cited in page 62.

WANG, M.; KHALILPOUR, R.; ABBAS, A. Thermodynamic and economic optimization of LNG mixed refrigerant processes. Energy Conversion and Management, Elsevier BV, v. 88, p. 947-961, dec 2014. Cited in page 65.

WANG, M.; ZHANG, J.; XU, Q. Optimal design and operation of a c3mr refrigeration system for natural gas liquefaction. Computers \& Chemical Engineering, Elsevier, v. 39, p. 84-95, apr 2012. Cited 4 times in pages 15, 61, 62, and 74 . 
WANG, M. E. X. Advanced Natural Gas Engineering: GULF PUB CO, 2009. ISBN 9781933762388. Cited in page 136.

WIESBERG, I. L. et al. Carbon dioxide management by chemical conversion to methanol: HYDROGENATION and BI-REFORMING. Energy Conversion and Management, Elsevier BV, v. 125, p. 320-335, oct 2016. Cited in page 80.

WILLERSRUD, A. et al. Short-term production optimization of offshore oil and gas production using nonlinear model predictive control. Journal of Process Control, Elsevier BV, v. 23, n. 2, p. 215-223, feb 2013. Cited 3 times in pages 62, 70, and 74.

$\mathrm{XIA}$, G. et al. Thermodynamic analysis and optimization of a solar-powered transcritical $\mathrm{CO} 2$ (carbon dioxide) power cycle for reverse osmosis desalination based on the recovery of cryogenic energy of LNG (liquefied natural gas). Energy, Elsevier BV, v. 66, p. 643-653, mar 2014. Cited in page 65.

XUE, Z. et al. Optimization strategies to explore multiple optimal solutions and its application to restraint system design. SAE International Journal of Passenger Cars - Mechanical Systems, SAE International, v. 5, n. 1, p. 540-551, apr 2012. Cited in page 85.

YAO, E. et al. Thermo-economic optimization of a combined cooling, heating and power system based on small-scale compressed air energy storage. Energy Conversion and Management, Elsevier, v. 118, p. 377-386, jun 2016. Cited 3 times in pages 68, 69, and 75. 


\section{APPENDIX A - MODELING AND \\ SIMULATION OF RB211G62 DLE 60HZ TURBINE IN GATECYCLE AND USING THIS DATA IN ASPEN HYSYS}

The RB211 gas turbines have also aero-derivative design. The DLE versions are in group of the gas turbines with the lowest pollutants emissions available on market. RB211 DLE $60 \mathrm{~Hz}$ gas turbine produces power of $27.90 \mathrm{MW}$ at full load at site temperature (SIEMENS). Figures A.1, A.2 and A.3, show, respectively, efficiency, exhausted mass flow rate and exhausted gas temperature by variation of the load (50\% to 100\%) for RB211G62 DLE $60 \mathrm{~Hz}$ comparing with other possible Gas Turbines and Internal Combustion Engines. Among of all Gas turbines, RB211 DLE $60 \mathrm{~Hz}$ has the highest efficiency in proposed condition (Figure A.1) .

Figure A.1 - Efficiency curves by variation of the load.

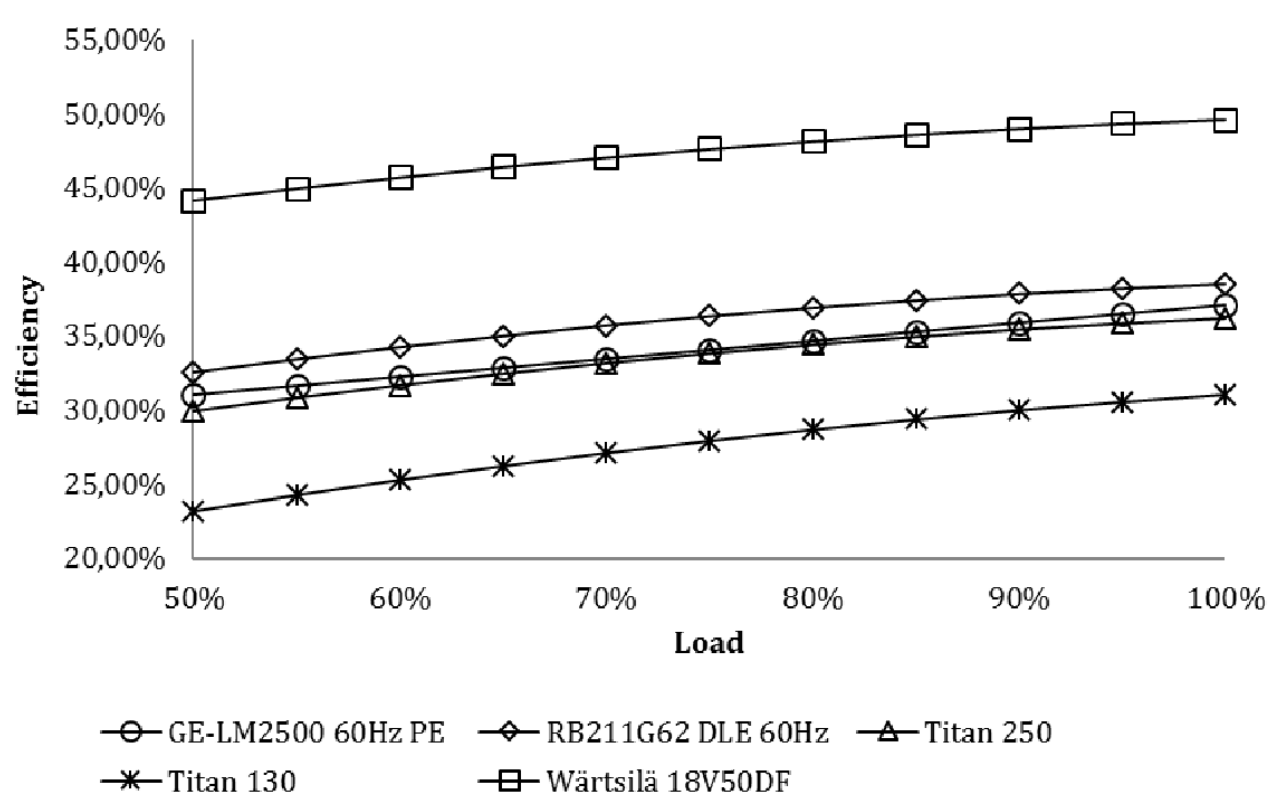

The Wärtsilä reciprocating engine showed best results over the entire range. Although Titan 250 turbine showed worse results compared to those from LM2500 turbine for low and high load, their efficiencies are very close in the range between $70 \%$ and $85 \%$.

Slight variation in exhausted gas mass flow rate as function of load is observed. It represents the increase of turbine inlet temperature (fuel consumption) with load. For the Wärtsilä engine, the variation of exhausted gas flow rate is considerably larger than the one for turbines. It 
APPENDIX A. Modeling and simulation of RB211G62 DLE 60Hz turbine in GATECYCLE and using this data 186

in Aspen Hysys

Figure A.2 - Curves of exhausted gas mass flow rate by variation of the load.

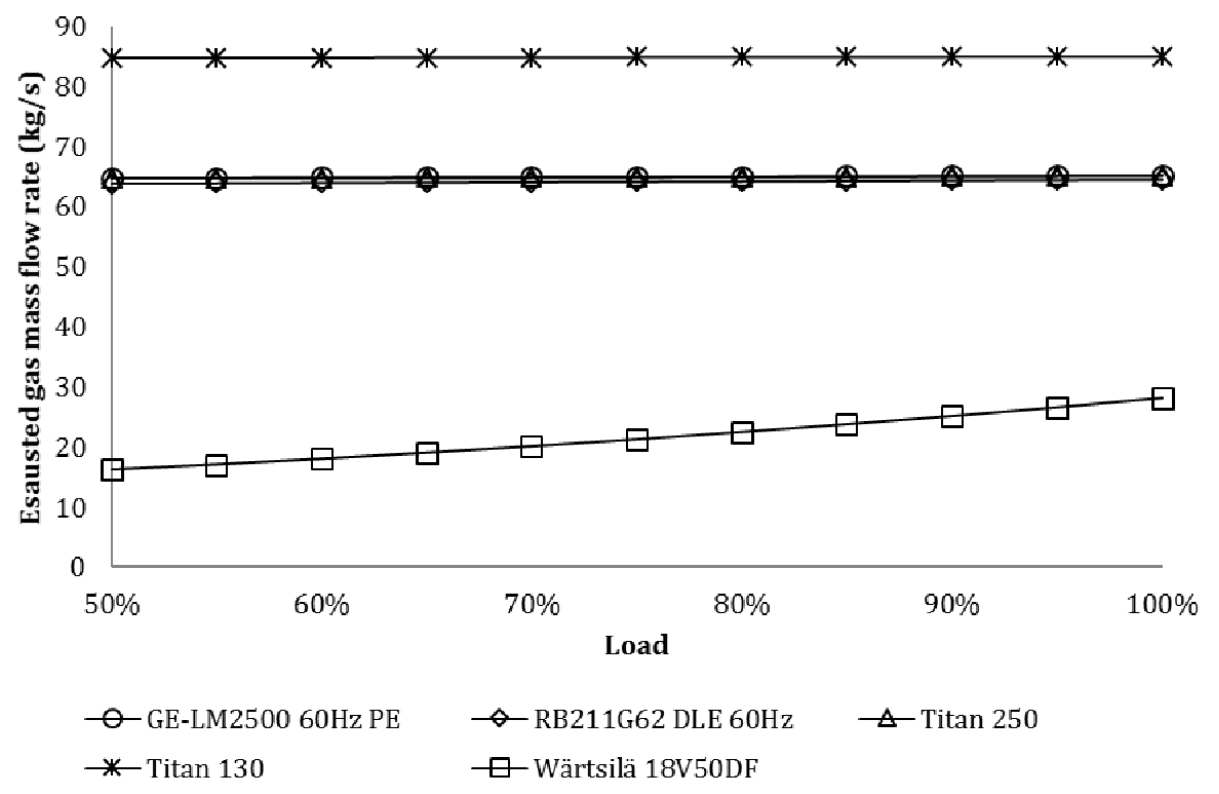

indicates that for this engine both, fuel and air consumption, increase. The exhausted gas temperature curves as function of load for the turbines showed similar patterns. See Figure A.3: The Titan 130 presented temperatures considerably lower than the other models of turbines (since this turbine has lower efficiency, it indicates lower temperature at expander inlet). In turn, RB211 DLE $60 \mathrm{~Hz}$ presents the adequate exhausted gas temperature for WHRU even in condition of $80 \%$ of total load.

Figure A.3 - Curve of the exhausted gas temperature by variation of the load.

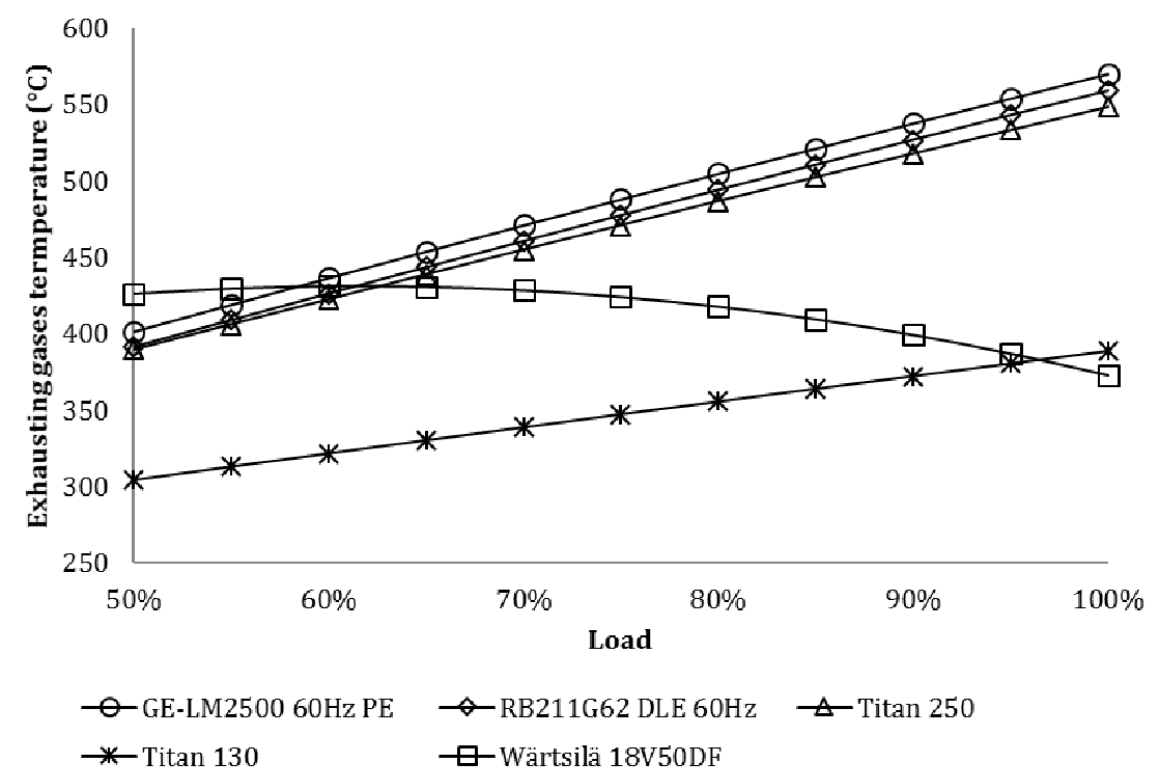

Figure A.4 shows the worksheet of various off-design calculation of RB211. In that, the input parameters are changing to find the converged condition of RB211. 
Figure A.4 - The used worksheet to calculate RB211 off-designs.

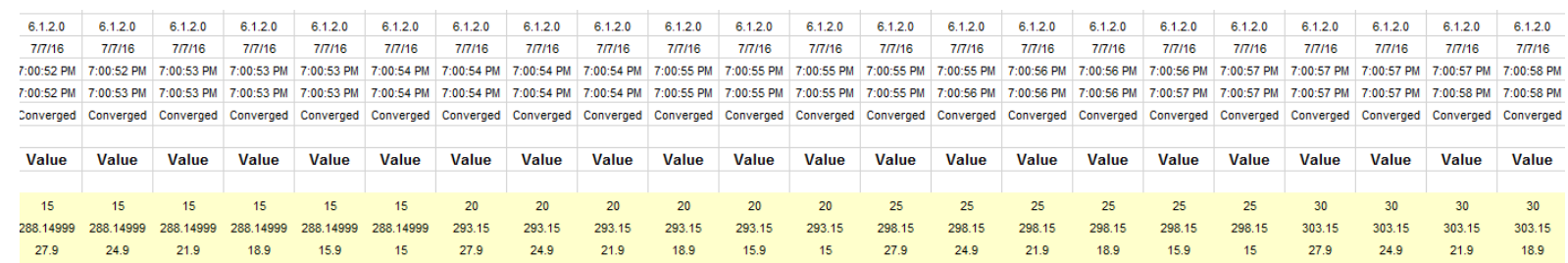

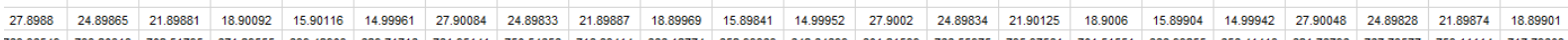
\begin{tabular}{llllllllllllllllllllllll}
82.436527 & 82314206 & 82.191831 & 671.29555 & 639.42903 & 629.71718 & 781.95141 & 750.54858 & 718.63414 & 686.12774 & 652.93383 & 642.84899 & 801.21539 & 768.55975 & 735.37561 & 701.51551 & 666.93255 & 656.41413 & 821.76796 & 787.73577 & 753.11114 & 717.79603 \\
\hline
\end{tabular}

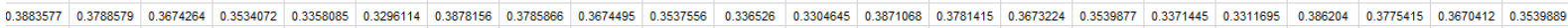

Figure A.5 shows the performance data of RB211 that regarding the variation of the power demand (and site temperature in specific condition), GATE CYCLE calculates the fuel consumption, efficiency, gas exhaust temperature and gas exhaust mass flow.

Figure A.5 - The performance data of RB211 that regarding the variation of input parameters

\begin{tabular}{c|c|c|c|c|} 
T_amb(C) & Net_PWR(MW) & T Out@Tur (C) & Flow_Out (Kg/s) & EFF \\
\hline 15 & 27.8988 & 763.385494 & 82.43652669 & 0.3883577 \\
15 & 24.89865 & 733.2031345 & 82.31420647 & 0.3788579 \\
\hline 15 & 21.89881 & 702.517947 & 82.19183113 & 0.3674264 \\
15 & 18.90092 & 671.2955485 & 82.06949517 & 0.3534072 \\
\hline 15 & 15.90116 & 639.4290256 & 81.94691508 & 0.3358085 \\
\hline 15 & 14.99961 & 629.7171794 & 81.91002923 & 0.3296114 \\
\hline 20 & 27.90084 & 781.9514052 & 79.83413709 & 0.3878156 \\
\hline 20 & 24.89833 & 750.5485799 & 79.71061989 & 0.3785866 \\
\hline 20 & 21.89887 & 718.6341444 & 79.58725232 & 0.3674495 \\
\hline 20 & 18.89969 & 686.1277357 & 79.46386112 & 0.3537556 \\
\hline 20 & 15.89841 & 652.9338338 & 79.3402258 & 0.336526 \\
\hline 20 & 14.99952 & 642.848995 & 79.30316671 & 0.3304645 \\
\hline 25 & 27.9002 & 801.215389 & 77.25723841 & 0.3871068 \\
\hline 25 & 24.89834 & 768.5597493 & 77.13267386 & 0.3781415 \\
\hline 25 & 21.90125 & 735.3756131 & 77.00829831 & 0.3673224 \\
\hline 25 & 18.9006 & 701.5155124 & 76.883718 & 0.3539877 \\
\hline 25 & 15.89904 & 666.9325521 & 76.75903533 & 0.3371445 \\
\hline 25 & 14.99942 & 656.4141262 & 76.72173211 & 0.3311695 \\
\hline 30 & 27.90048 & 821.7679606 & 74.62002618 & 0.386204 \\
\hline 30 & 24.89828 & 787.7357747 & 74.49416227 & 0.3775415 \\
\hline 30 & 21.89874 & 753.111141 & 74.36847949 & 0.3670412 \\
\hline 30 & 18.89901 & 717.79603 & 74.24302508 & 0.3539889 \\
\hline 30 & 15.90068 & 681.7380534 & 74.11733442 & 0.3375739 \\
\hline 30 & 15.00064 & 670.7516574 & 74.07952721 & 0.331784 \\
\hline & & & & \\
\hline 5 & & & & \\
\hline 5 & & & &
\end{tabular}


APPENDIX A. Modeling and simulation of RB211G62 DLE 60Hz turbine in GATECYCLE and using this data

Figure A.6 shows how the performance data of RB211 is applied in Aspen Hysys using Spreadsheet tool of it.

Figure A.6 - Applied Gas turbine RB211 performance data in Aspen HYSYS.

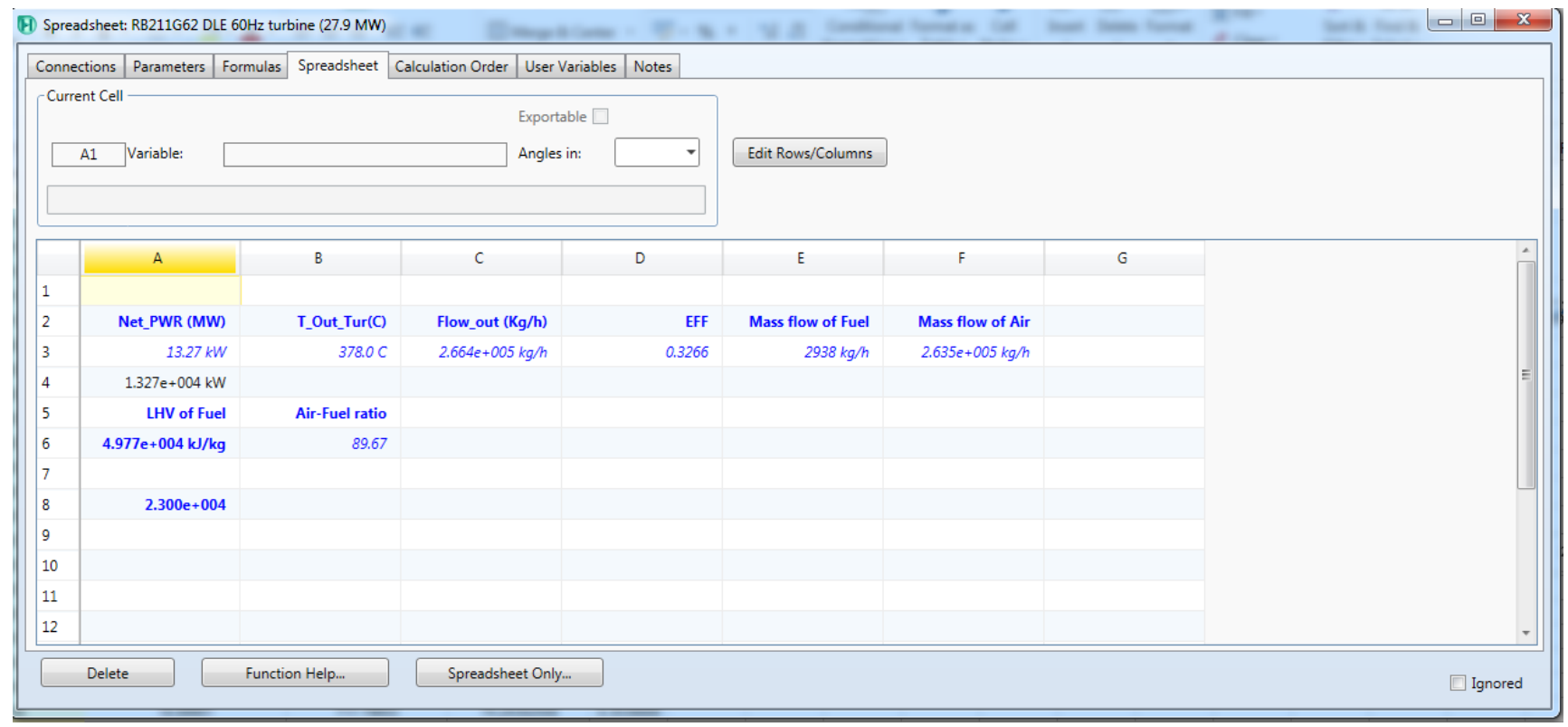




\section{APPENDIX B - MODELING AND \\ SIMULATION SIMULATOR ILLUSTRATION OF FPSO BY ASPEN HYSYS}

Figure B.1 - General scheme of proposed FPSO

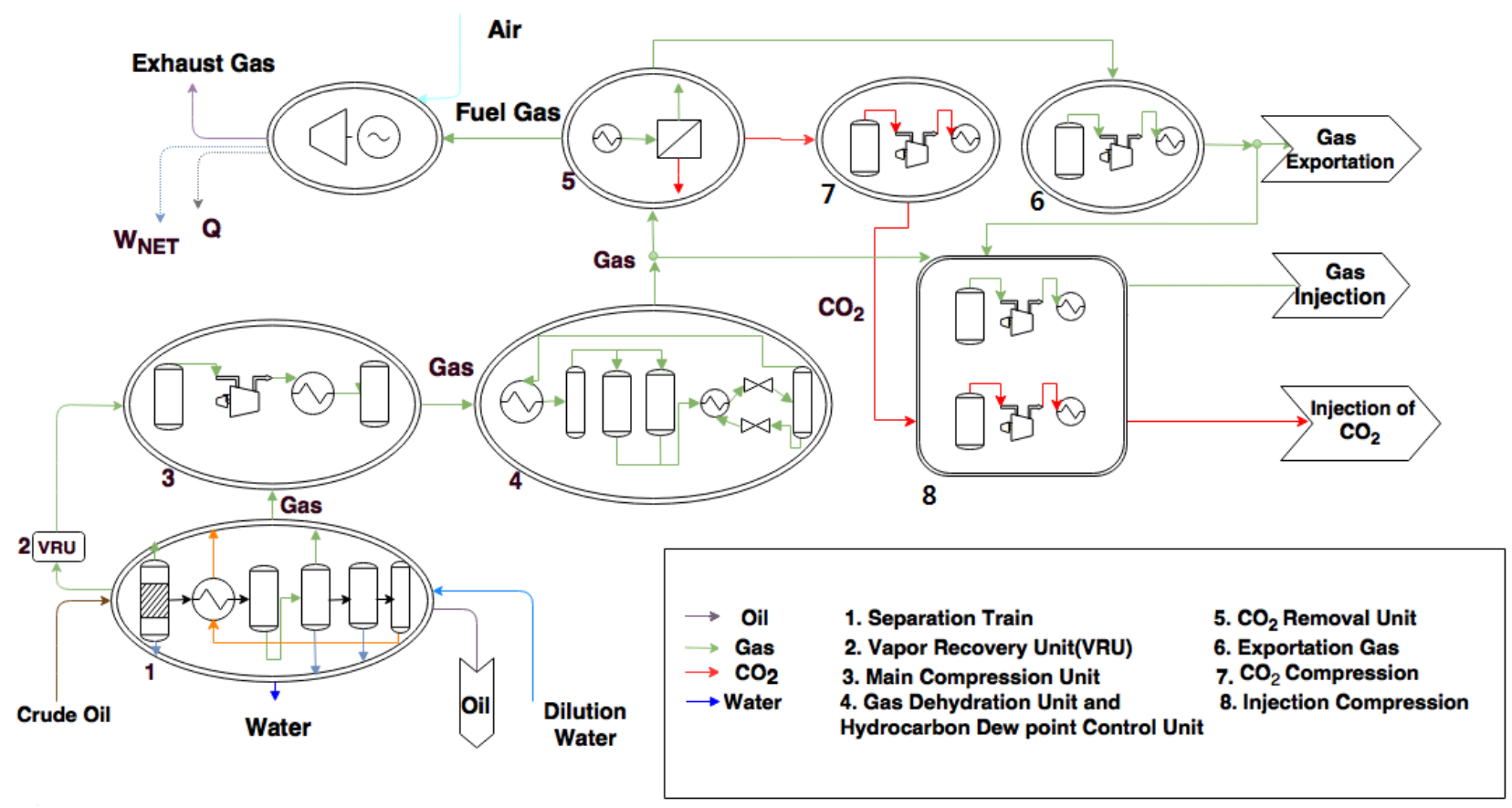




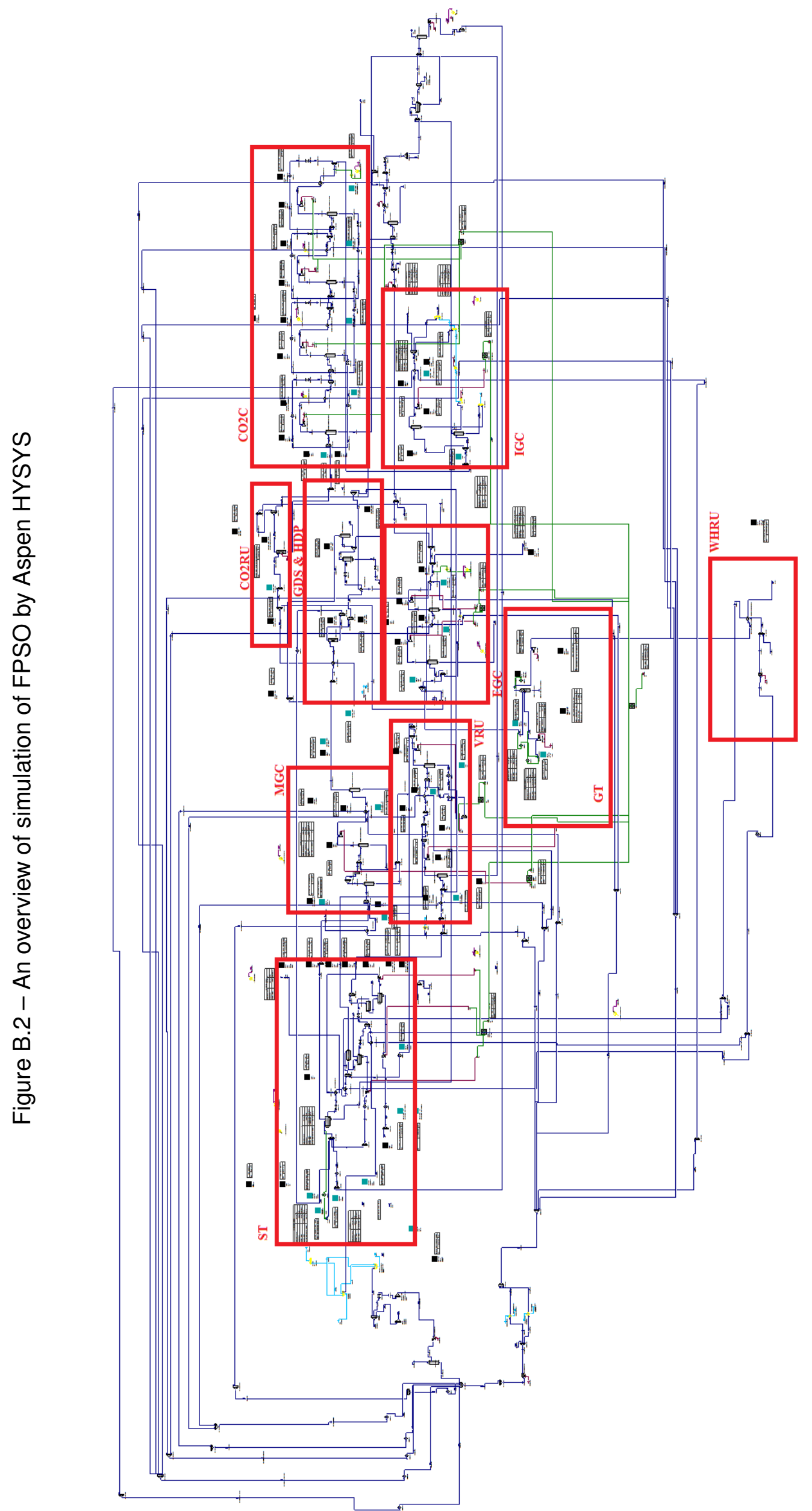


Figure B.3 - Configuration of FPSO plant and utilities plant in this study.

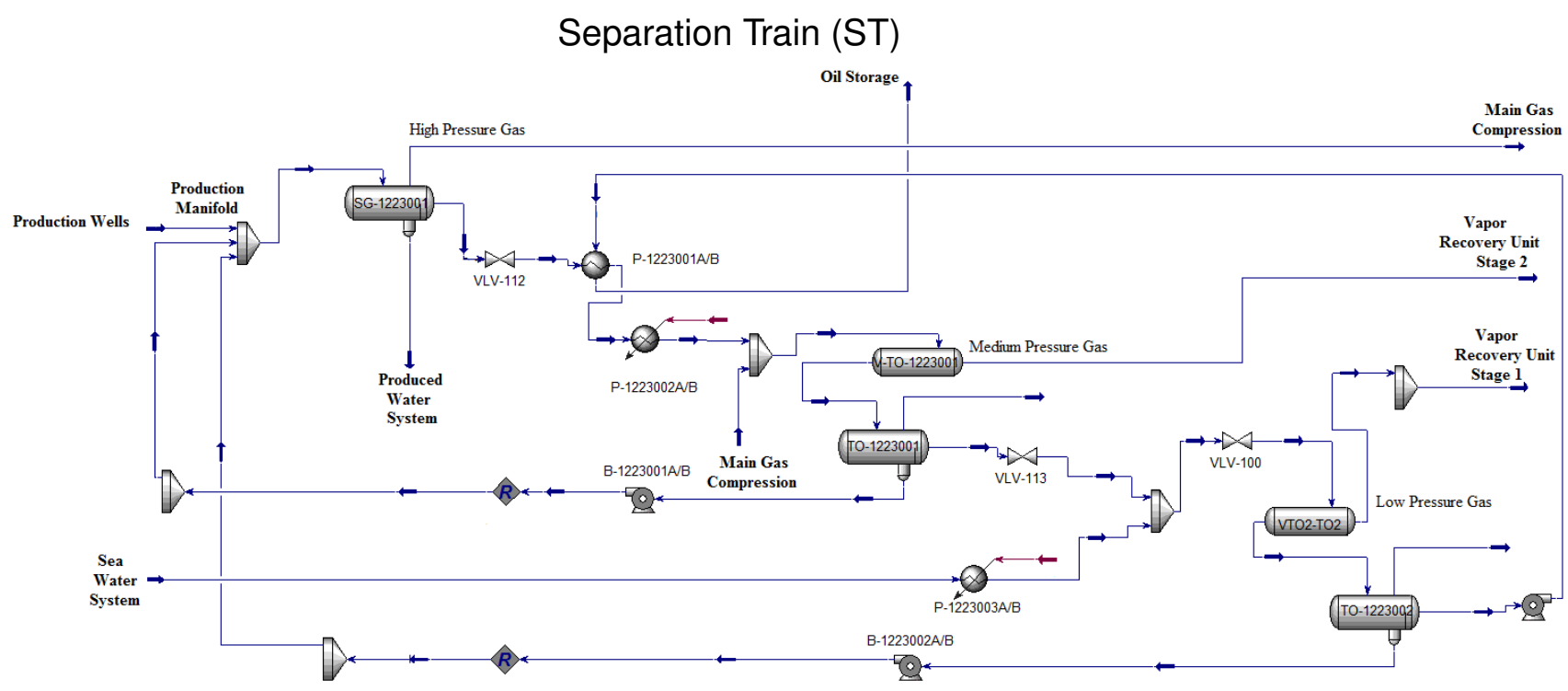

Figure B.4 - Vapor Recovery Unit (VRU)

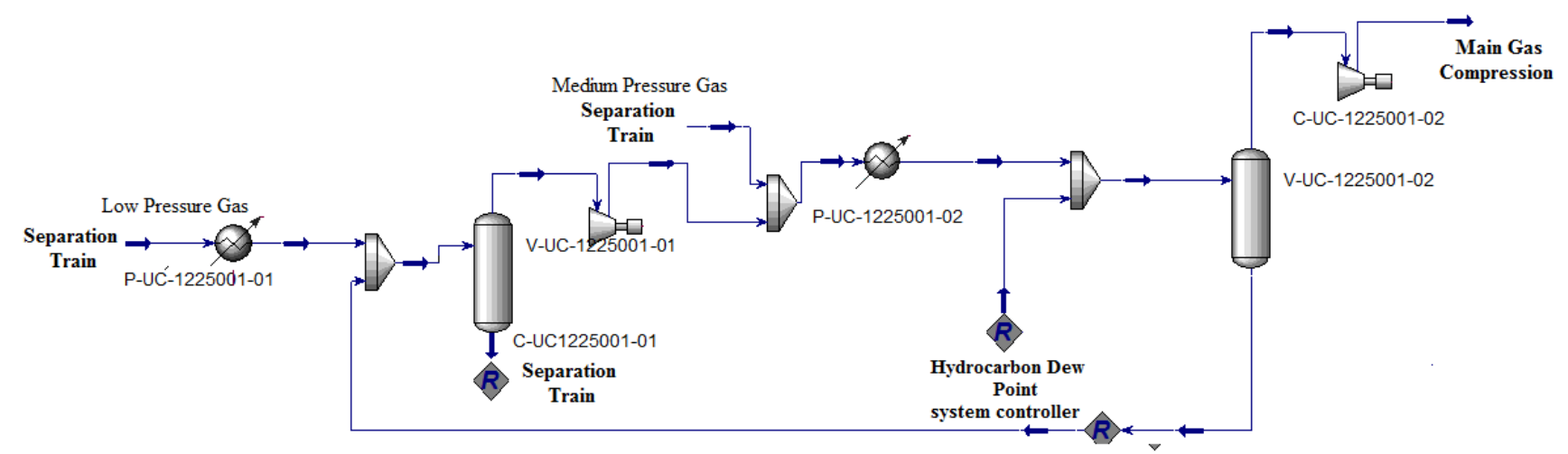


Figure B.5 - Main Gas Compression Unit (MGC)

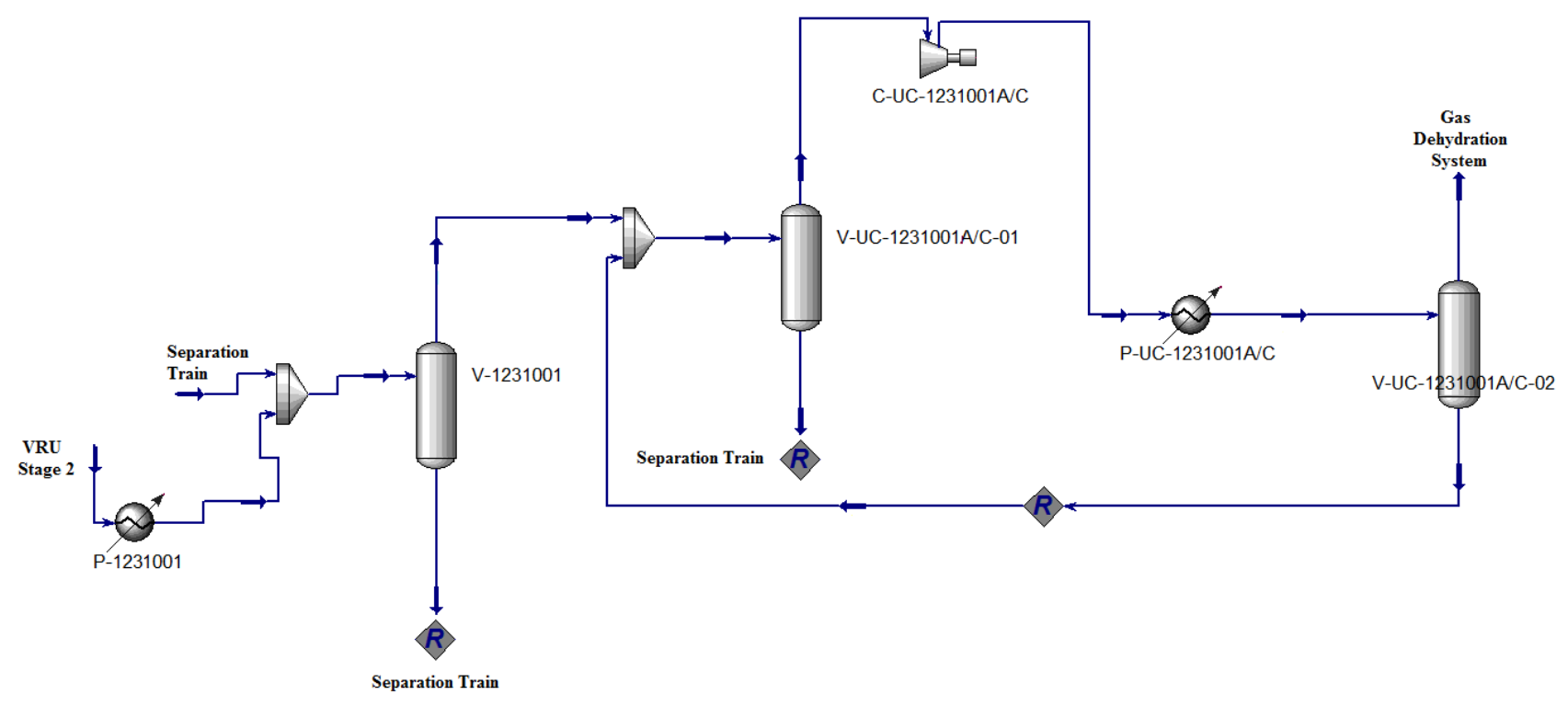

Figure B.6 - Gas Dehydration System and $\mathrm{CO}_{2}$ Removal Unit (GDS\&CO2RU)

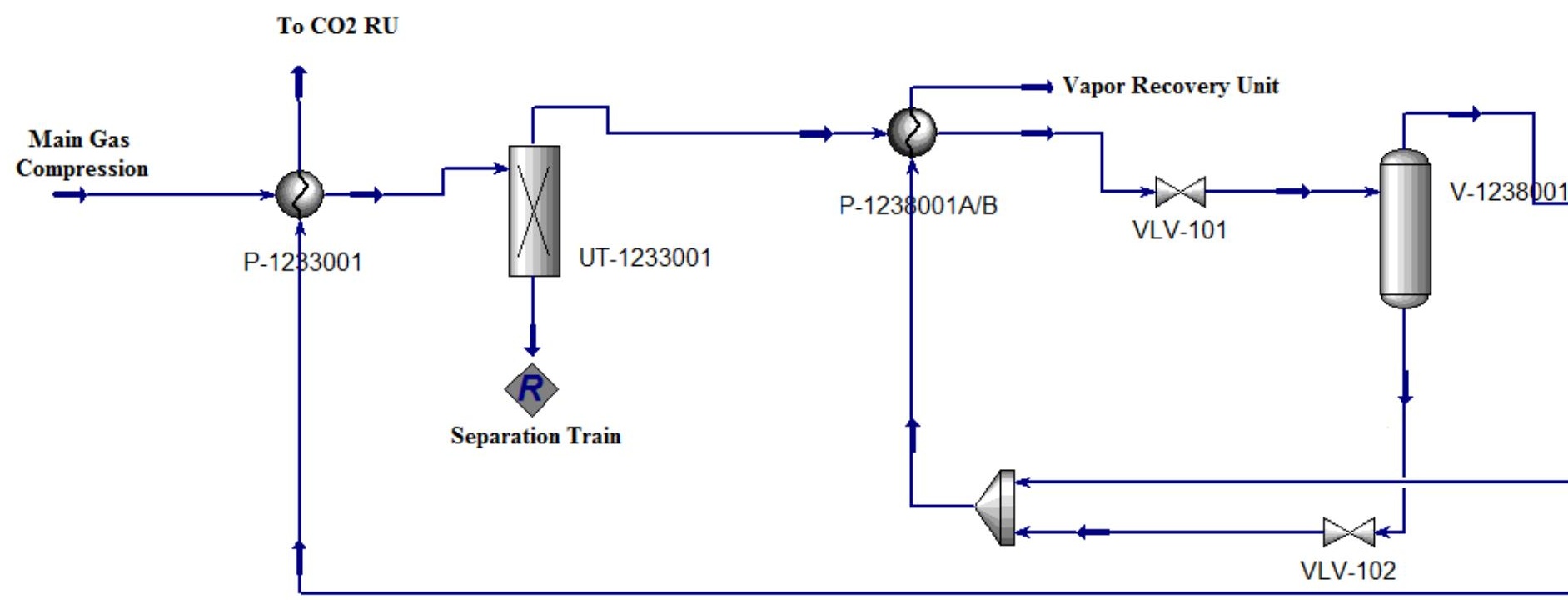


Figure B.7 $-\mathrm{CO}_{2}$ Compression(CO2C)

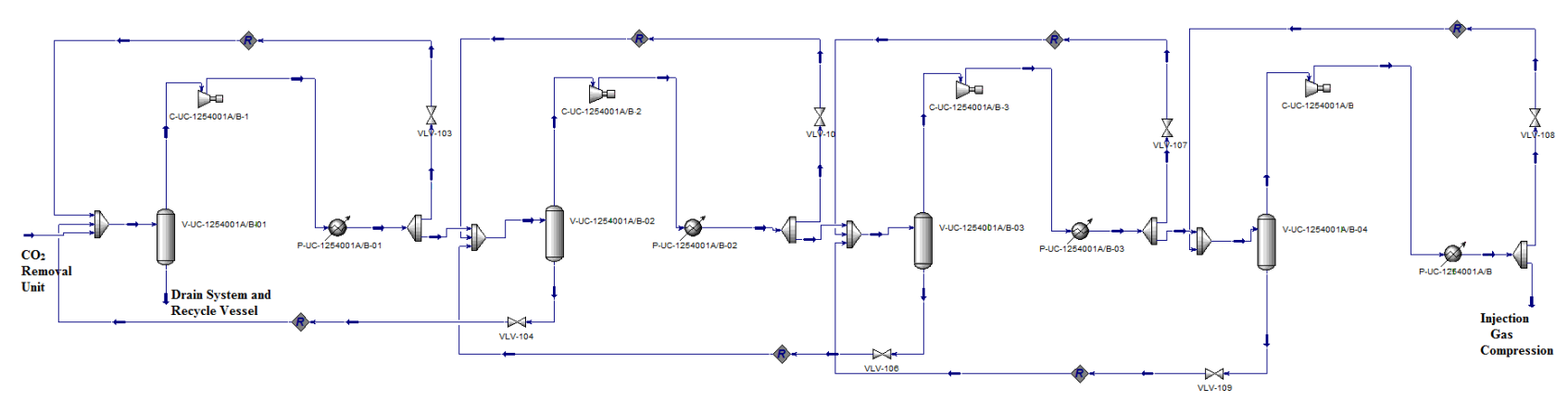


ஊ

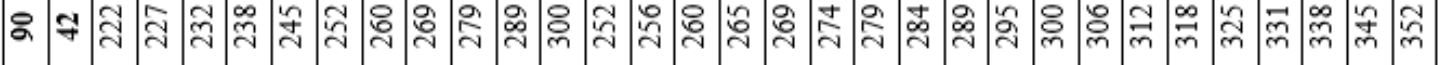

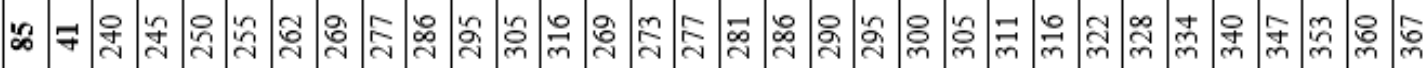

\& م

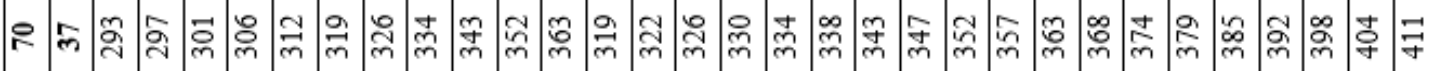

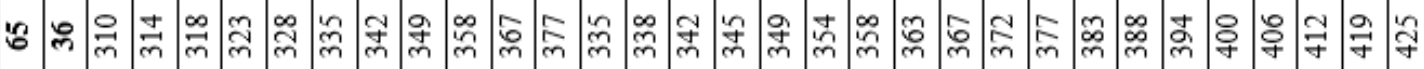

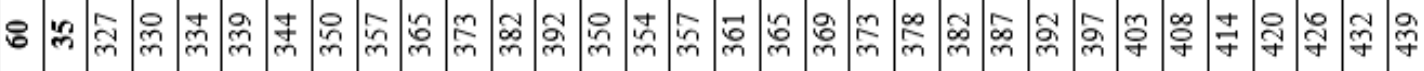

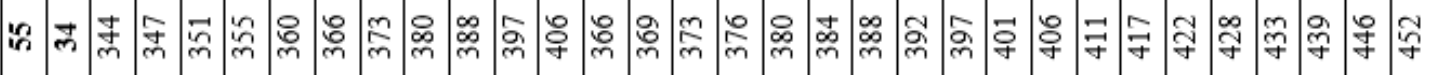

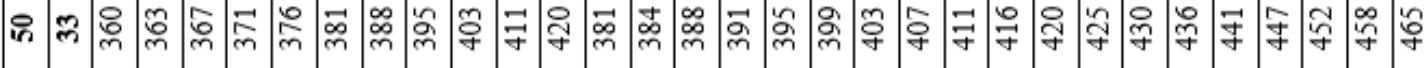

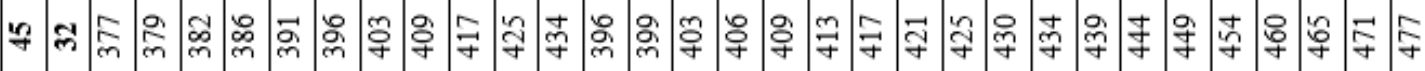

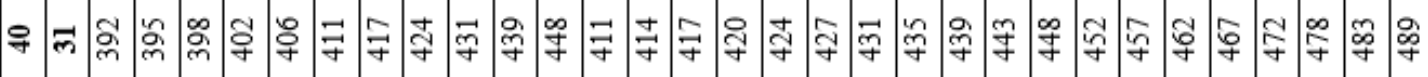
๗

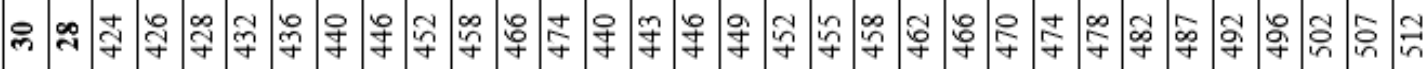

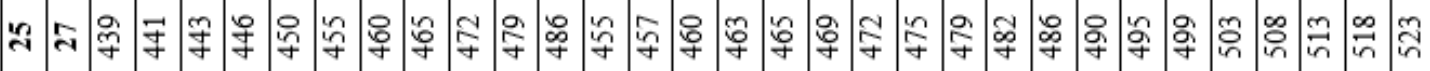

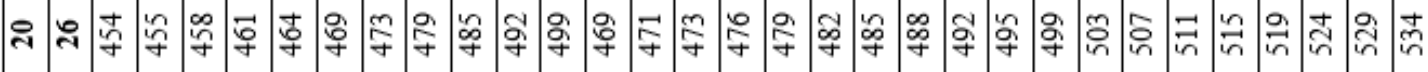

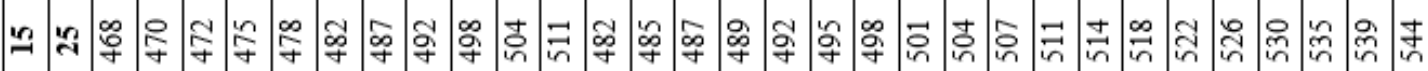

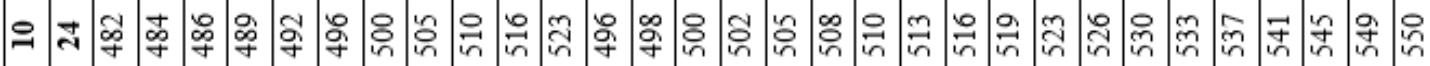

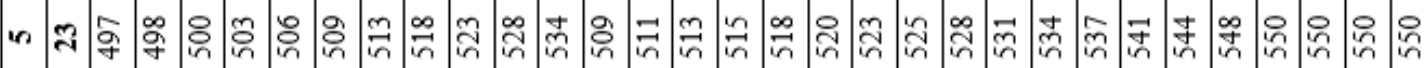

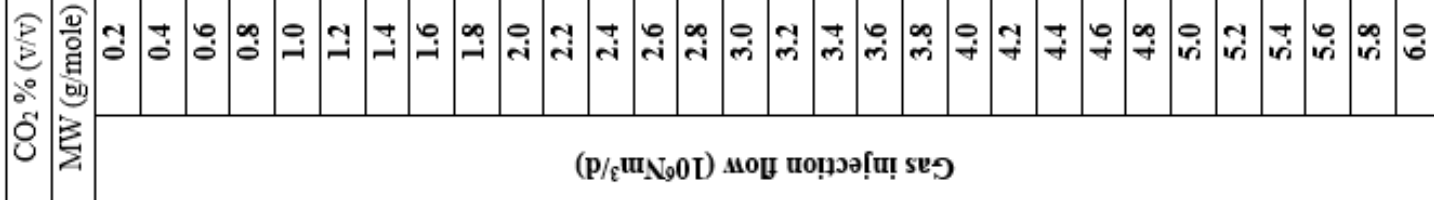


Figure B.8 - Exportation Gas Compression(EGC)

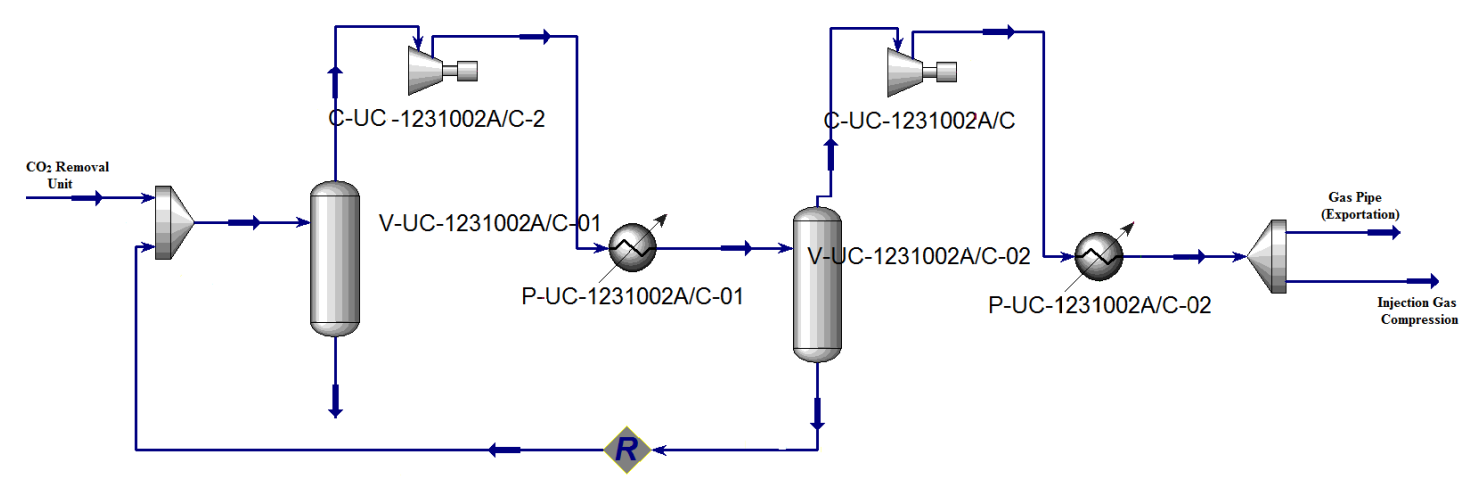

Figure B.9 - Injection Gas Compression(IGC)

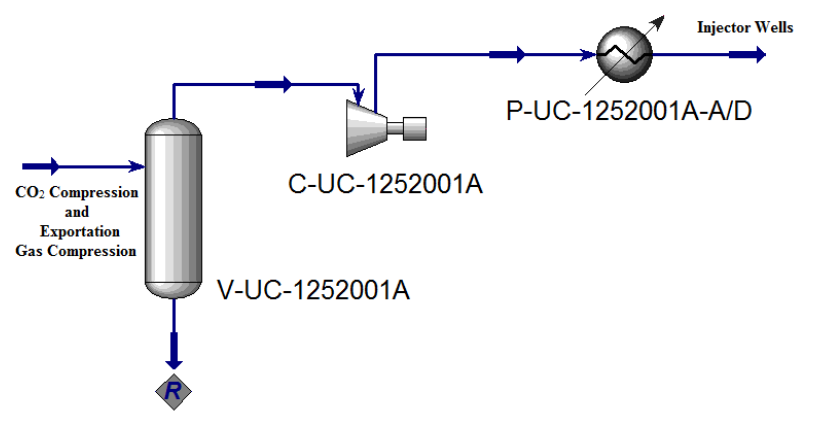

Figure B.10 - Gas Turbine and Waste Heat Recovery Unit (GT\&WHRU)

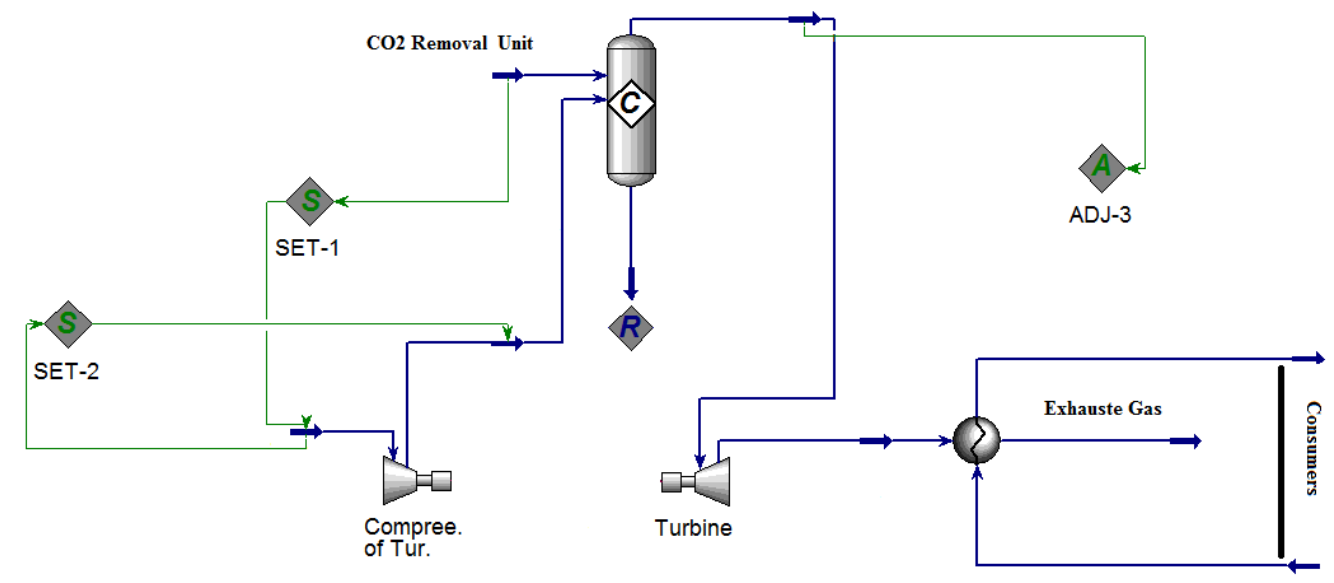





\section{APPENDIX C - COUPLING MODEFRONTIER WITH ASPEN HYSYS}

The Hysys is defined as simulator in MF and it is called in MF by a text file. The engine of Hysys opens in background and then the variables replace in Script manager of Hysys. Then, the results are copied in output file of MF by the Hysys Report Writer. The new inputs for simulation are generated by MF (for screening analysis and/or optimization algorithm) and they are inserted to Hysys to check it for technical constraints and numerical convergence.

Figure C.1 - Couple ModeFrontier with ASPEN HYSYS
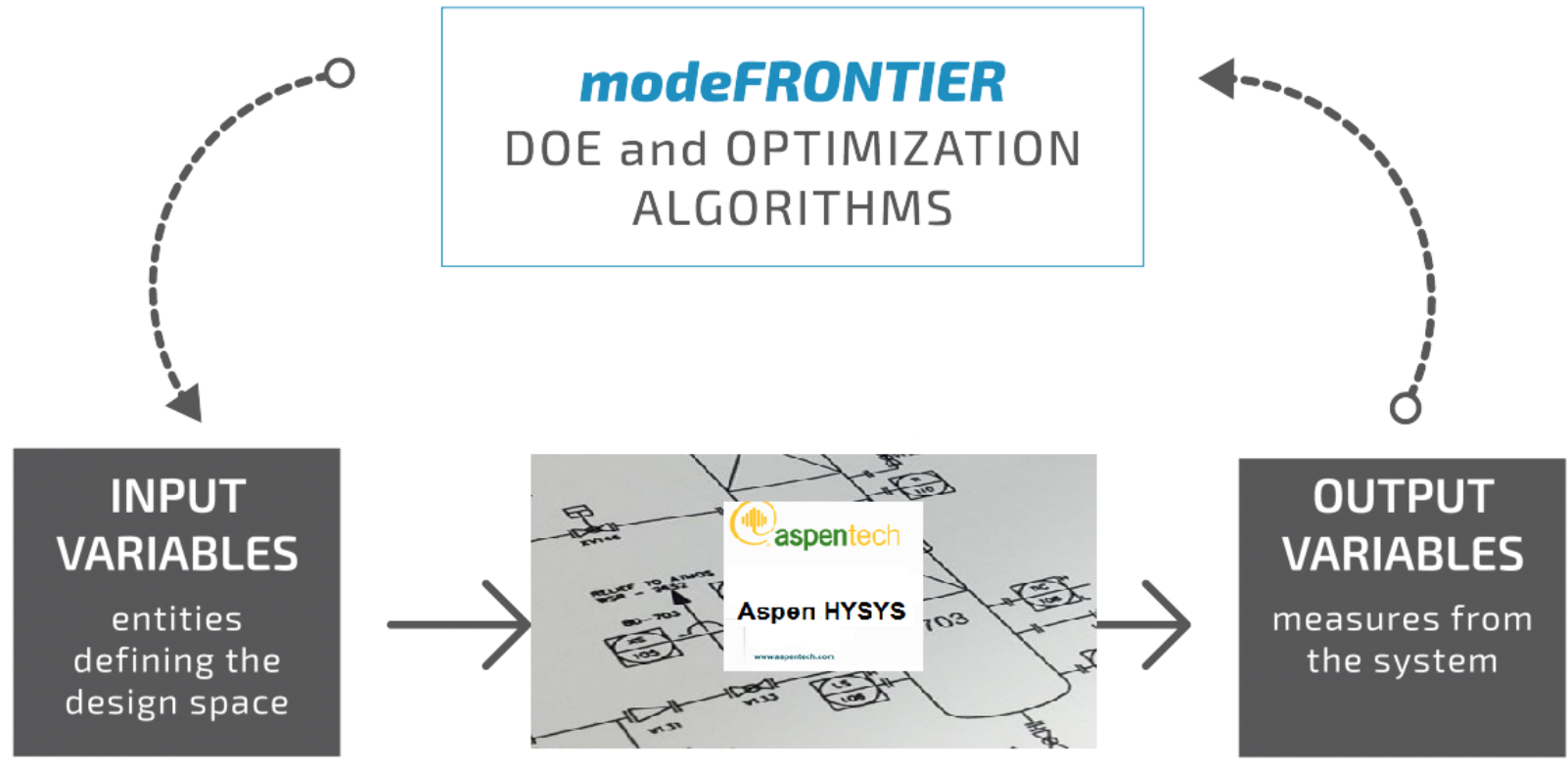

Figure C.2 shows a work flow of ModeFrontier for a optimization procedure. In that, the input parameters, technical constraints and optimization method are connected to achieve the stopping criteria of optimization algorithm. Several output parameter can be monitored during the optimization process and making the objective by calculation of output variables. 
Figure C.2 - Work flow of ModeFrontier for an optimization procedure

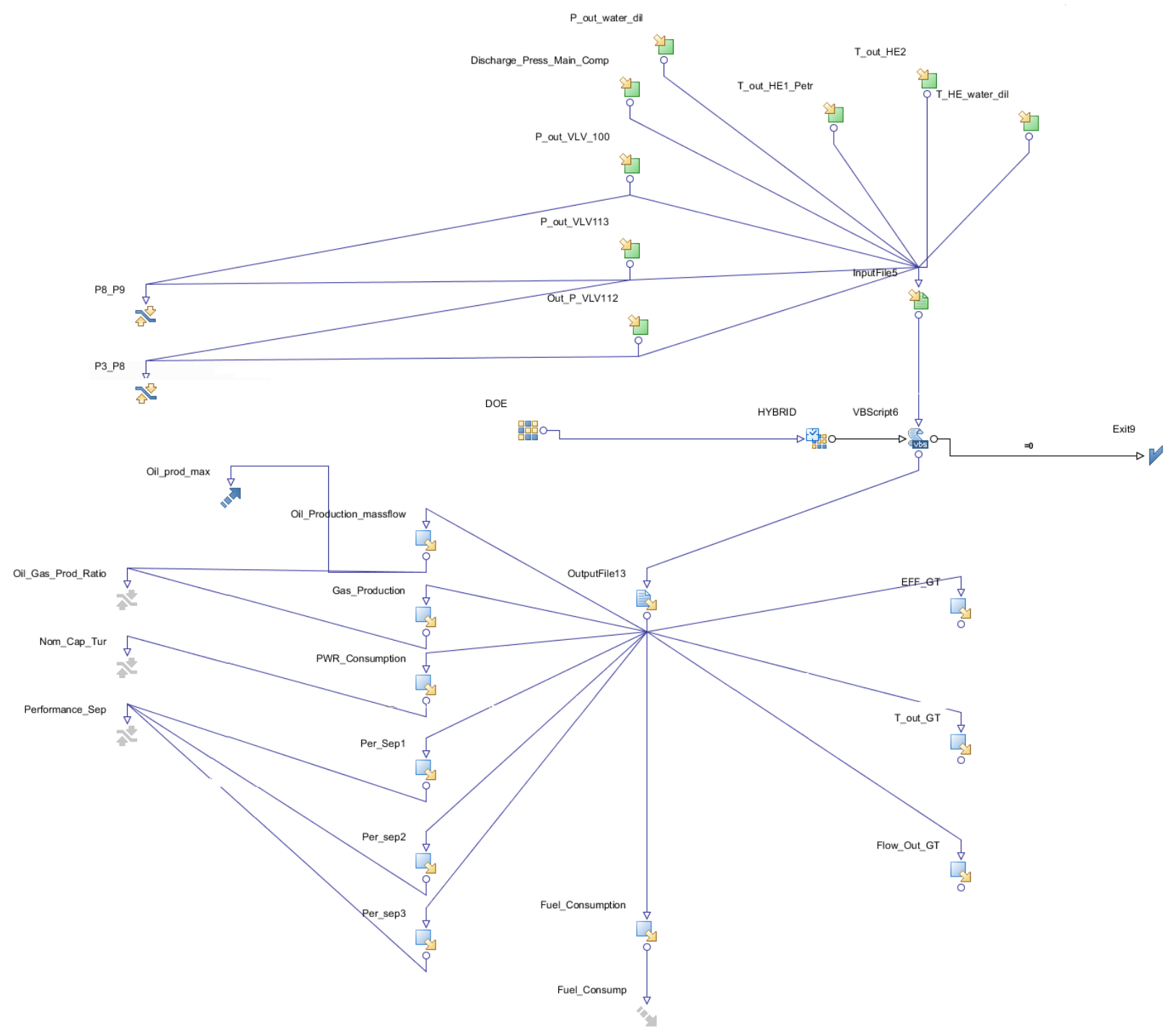


Figure C.3 displays the results of SS-ANOVA screening analysis of operation mode 1 in hydrocarbon liquids recovery.

Figure C.3 - Results of SS-ANOVA of hydrocarbon liquids recovery in ModeForntier

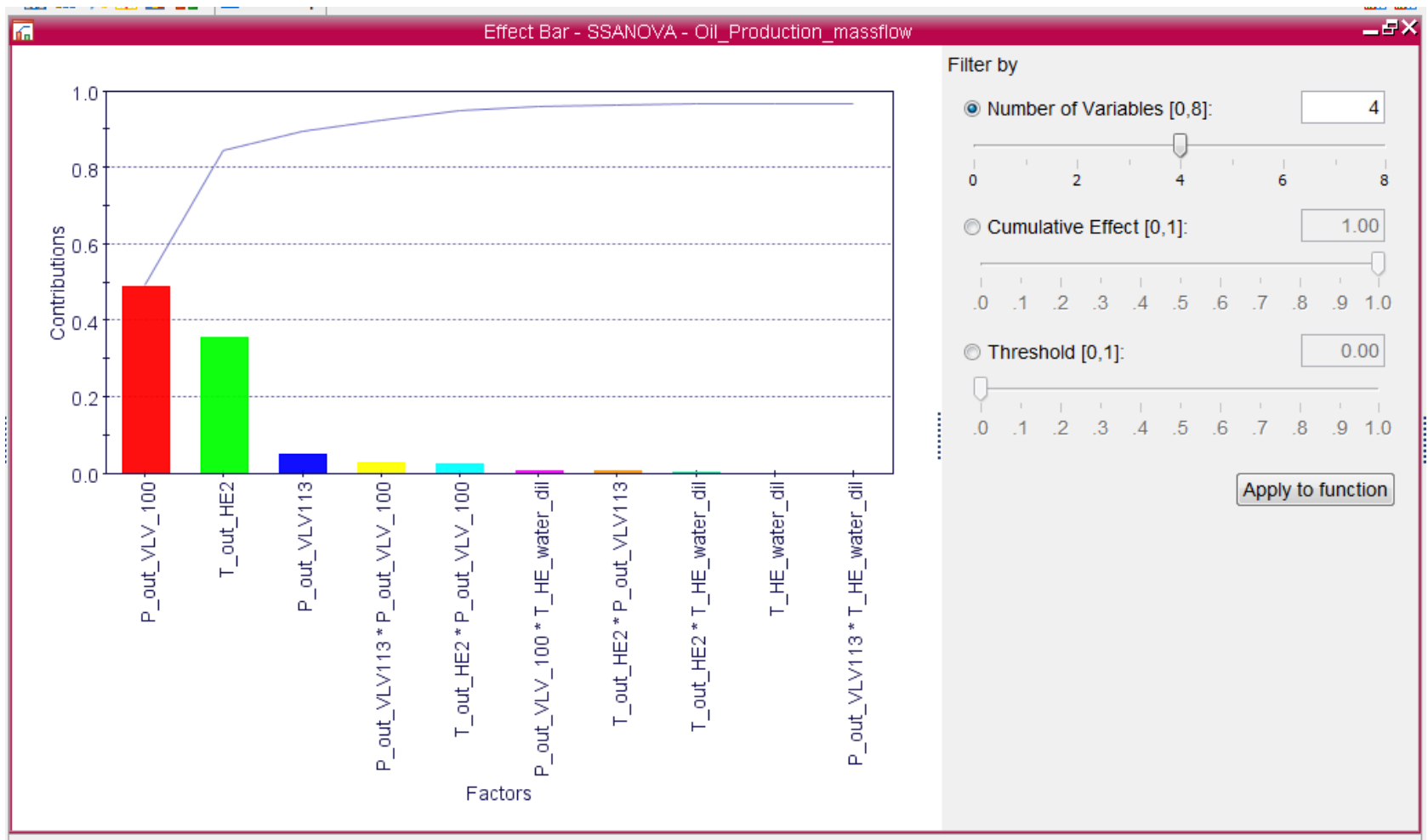





\section{APPENDIX D - CONVERGED OBJECTIVE FUNCTIONS}

Figure D.1 - Hybrid algorithm convergence curve for fuel consumption minimization- mode 1

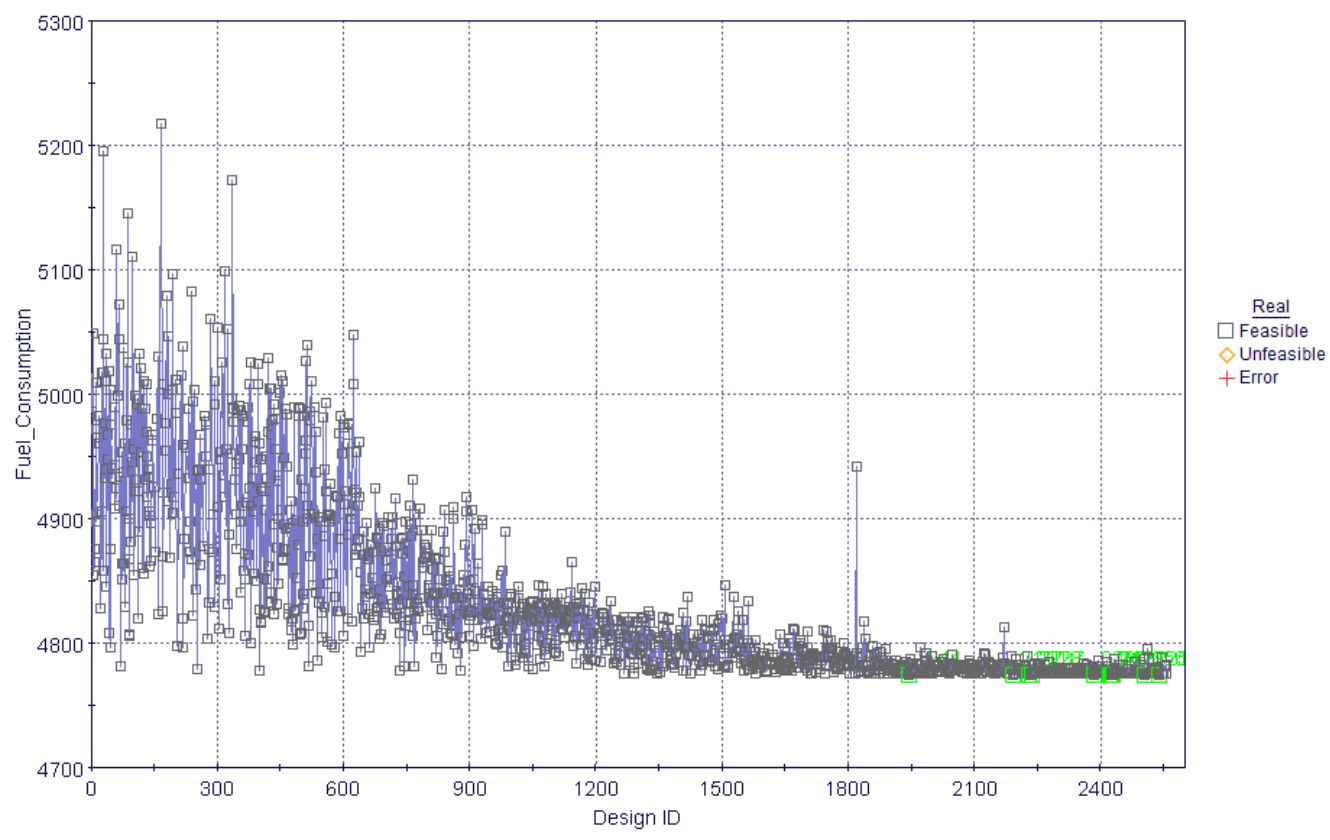


Figure D.2 - Hybrid algorithm convergence curve for fuel consumption minimization- mode 2

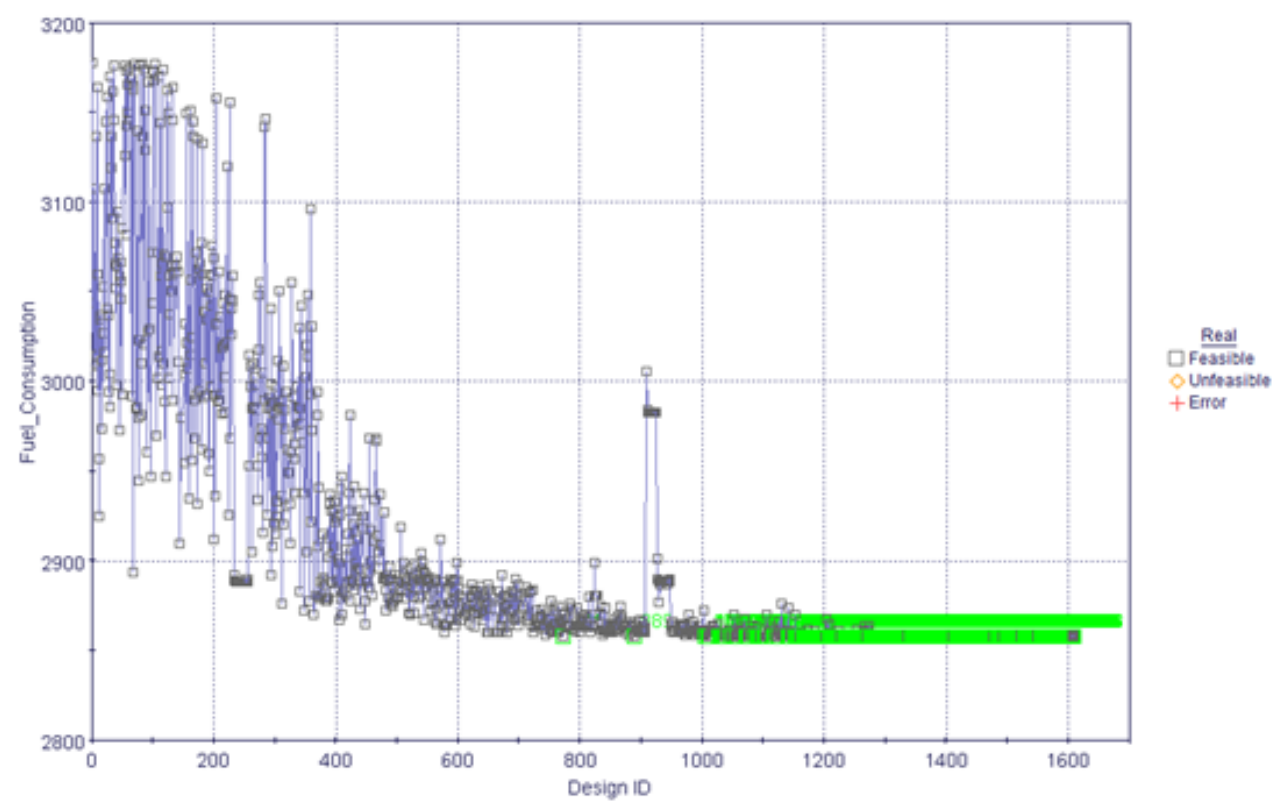

Figure D.3 - Hybrid algorithm convergence curve for fuel consumption minimization- mode 3

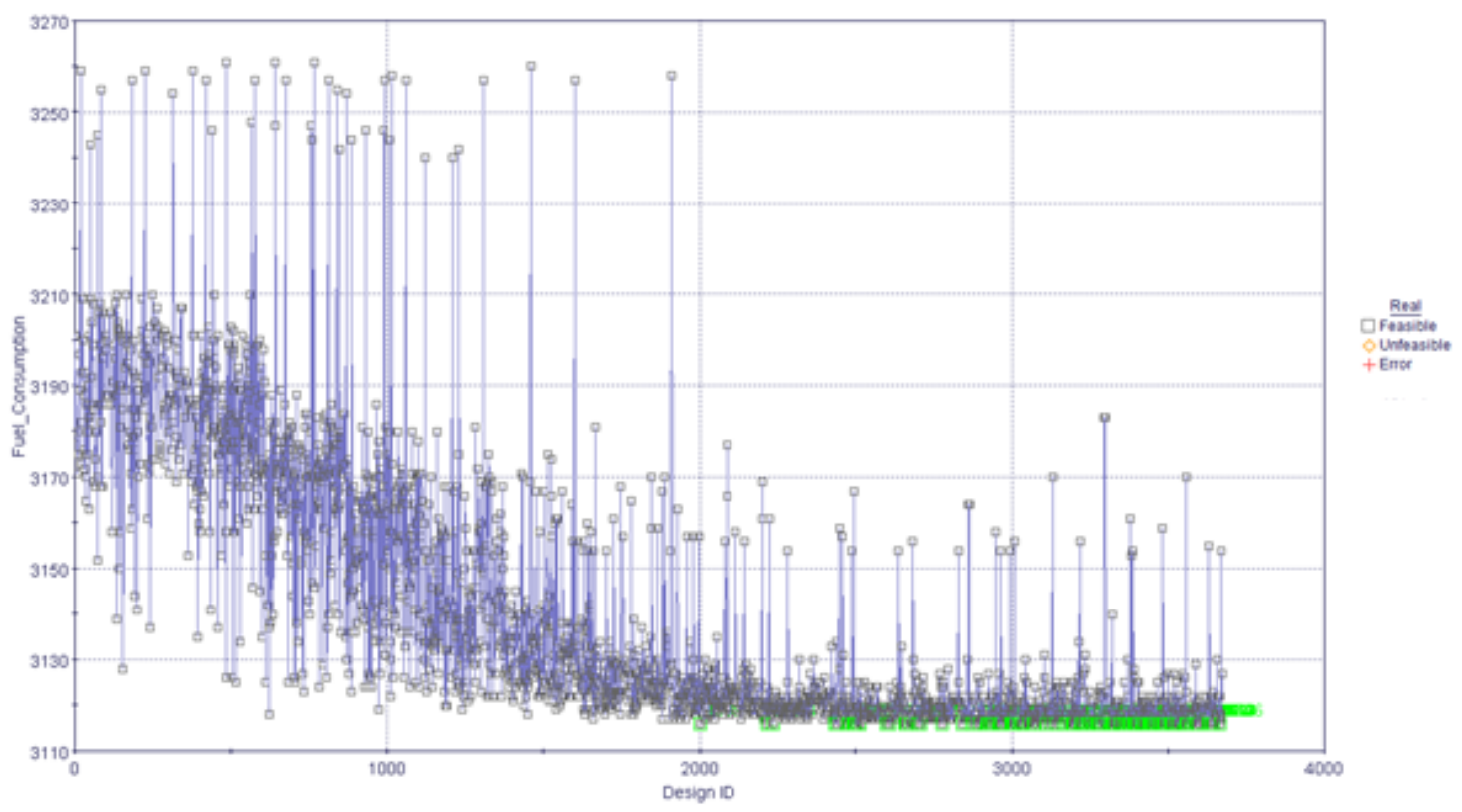


Figure D.4-Hybrid algorithm convergence curve for hydrocarbon liquids recovery maximization - mode 1

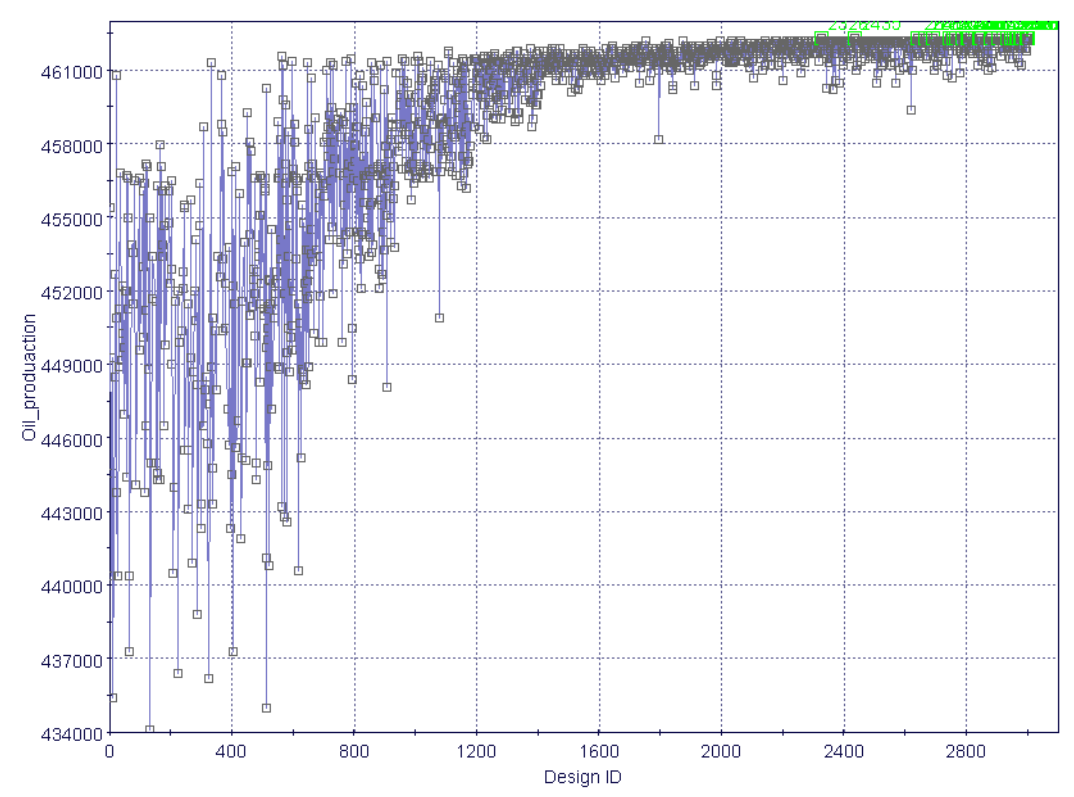

$\square$ Feasible

$\checkmark$ Unfeasible

+Error

Virtual

Feasible
Unfeasible

Figure D.5 - Hybrid algorithm convergence curve for hydrocarbon liquids recovery maximization - mode 2

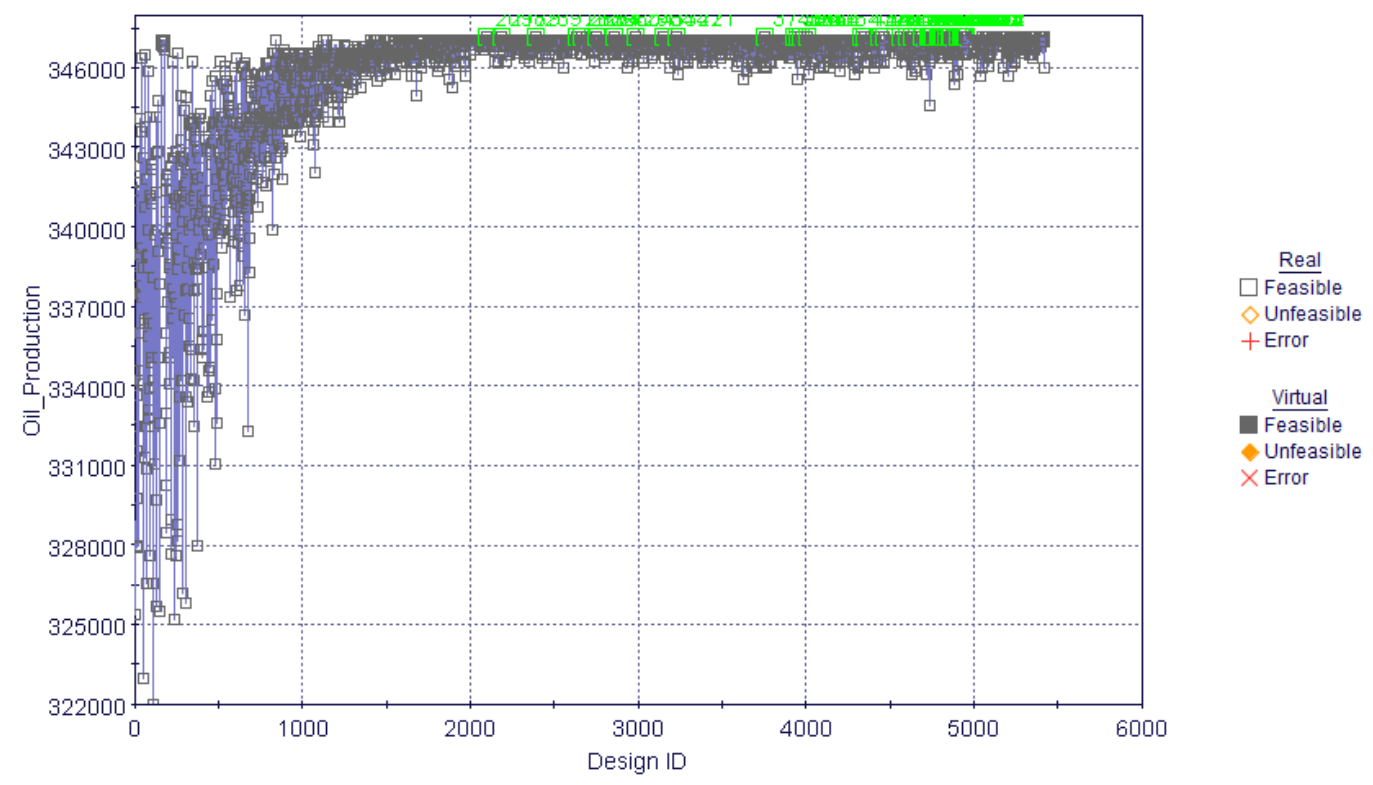


Figure D.6-Hybrid algorithm convergence curve for hydrocarbon liquids recovery maximization - mode 3

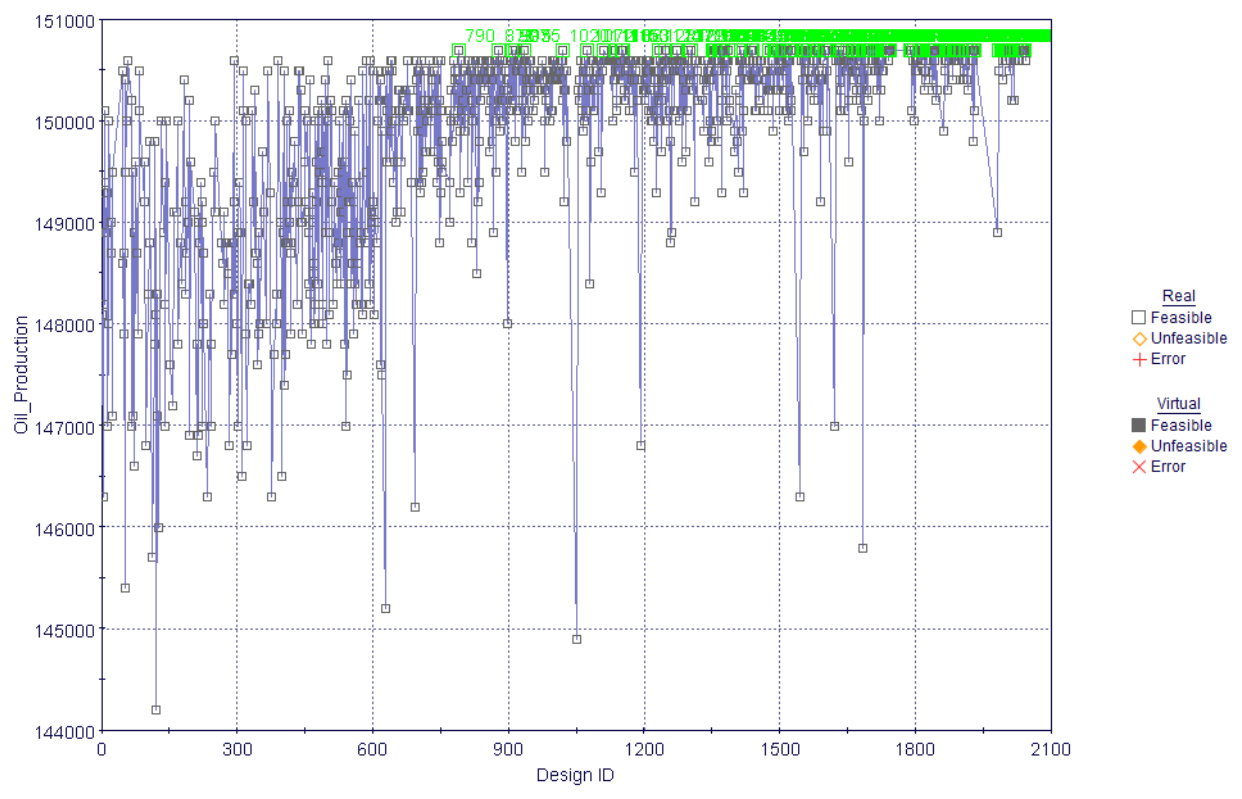

